\title{
Touching Light: A Framework for the Facilitation of Music-Making in Mixed Reality
}

Ian Thomas Riley

West Virginia University, ir0016@mix.wvu.edu

Follow this and additional works at: https://researchrepository.wvu.edu/etd

Part of the Art Education Commons, Audio Arts and Acoustics Commons, Composition Commons, Fine Arts Commons, Music Education Commons, Music Pedagogy Commons, Music Performance Commons, Music Practice Commons, Online and Distance Education Commons, and the Other Computer Engineering Commons

\section{Recommended Citation}

Riley, Ian Thomas, "Touching Light: A Framework for the Facilitation of Music-Making in Mixed Reality" (2021). Graduate Theses, Dissertations, and Problem Reports. 8075.

https://researchrepository.wvu.edu/etd/8075

This Dissertation is protected by copyright and/or related rights. It has been brought to you by the The Research Repository @ WVU with permission from the rights-holder(s). You are free to use this Dissertation in any way that is permitted by the copyright and related rights legislation that applies to your use. For other uses you must obtain permission from the rights-holder(s) directly, unless additional rights are indicated by a Creative Commons license in the record and/ or on the work itself. This Dissertation has been accepted for inclusion in WVU Graduate Theses, Dissertations, and Problem Reports collection by an authorized administrator of The Research Repository @ WVU.

For more information, please contact researchrepository@mail.wvu.edu. 
Touching Light: A Framework for the Facilitation of Music-Making in Mixed Reality

\author{
Ian Riley
}

Doctoral document submitted

to the College of Creative Arts

at West Virginia University

in partial fulfillment of the requirements for the degree of

Doctor of Musical Arts in

Percussion Performance

George R. Willis, MM, MBA, Chair, Research Advisor

Michael B. Vercelli, DMA

Andrea Houde, MM

Rhonda Reymond, $\mathrm{PhD}$

School of Music

Morgantown, West Virginia

2021

Keywords: Music, Percussion, Performance Practice, Pedagogy, Virtual Reality, Mixed Reality, VR/MR, HoloLens, Microsoft, Live Electronics

Copyright 2021 Ian Riley 


\begin{abstract}
Touching Light: A Framework for the Facilitation of Music-Making in Mixed Reality Ian Riley
\end{abstract}

Drawing upon the historical development of analog and digital technologies alongside the proliferation of computer-assisted performance practices, this research seeks to develop a framework for integrating Mixed Reality applications to live musical performance, specifically through the creation of a Microsoft HoloLens 2 Mixed Reality application in order to facilitate a live performance of an original musical composition for percussion and real-time Mixed Reality environment. Mixed Reality enables a performer to interact with virtual (holograms, VSTs, etc.) and physical (vibraphone, tuned drums, microphones, etc.) objects simultaneously. Tandem to the development of the conceptual framework was the composition of an original score for solo percussionist and Mixed Reality environment. The score was composed to uniquely serve this interaction between virtual and physical spaces by employing a custom HoloLens 2 application.

A proposal of idiomatic performance practices as a 'framework' for music-making in Mixed Reality functions as the focus of this project. This framework was derived from iterative experimentation with the Microsoft HoloLens 2 and facilitated by the author's practical music performance experience. 


\section{Dedication}

This document is humbly dedicated to my wife Kristen, without whom this work would not have been possible. Thank you for your faithful, tireless, and joyous support.

Here's to the next adventure.

Page | iii 


\section{Table of Contents}

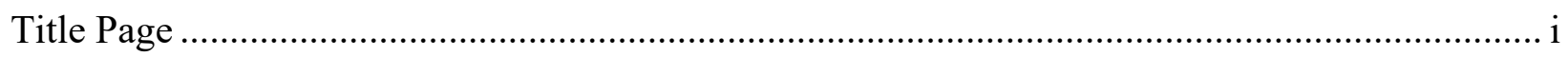

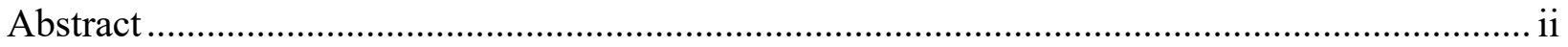

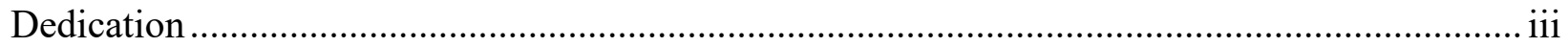

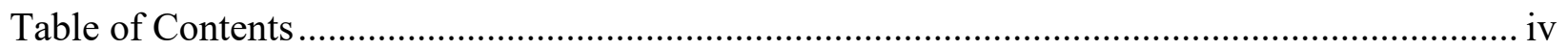

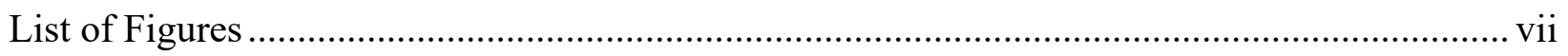

List of Media Examples ..................................................................................................

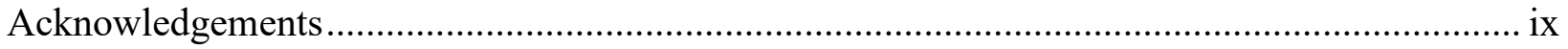

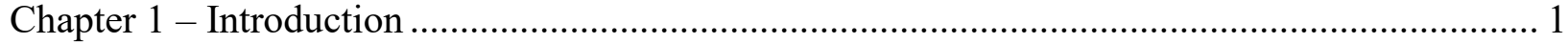

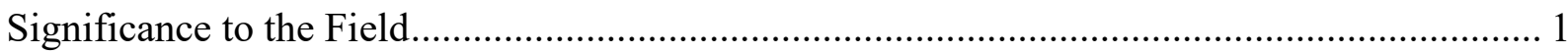

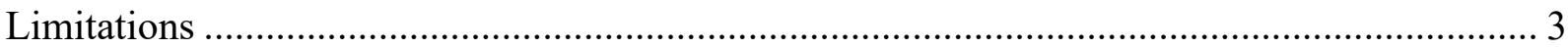

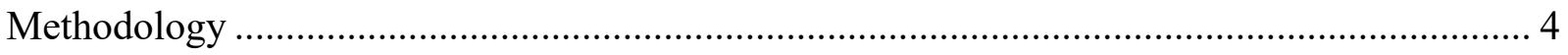

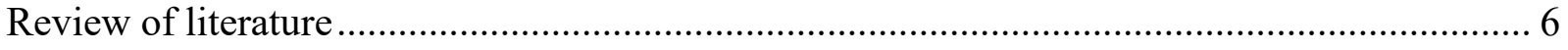

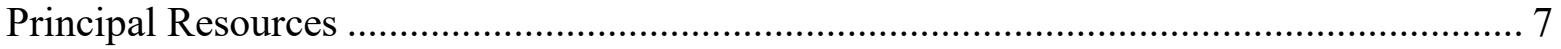

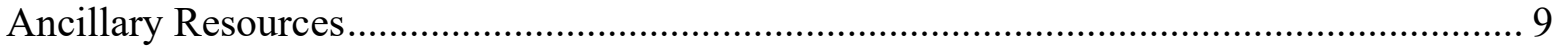

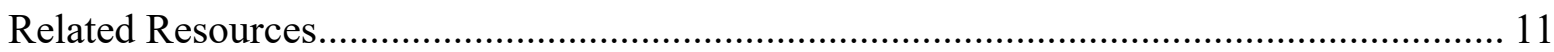

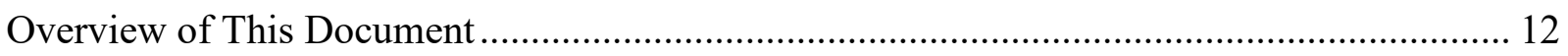

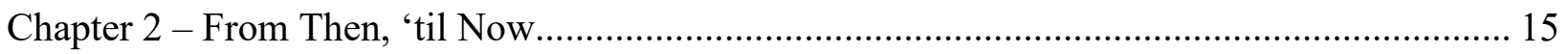

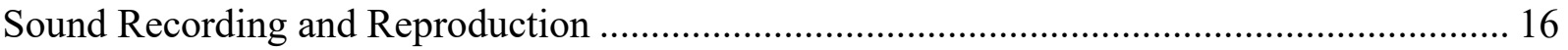

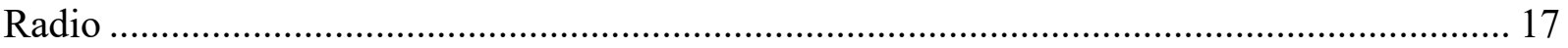

Synthesized Audio and Music Visualization .................................................................... 18

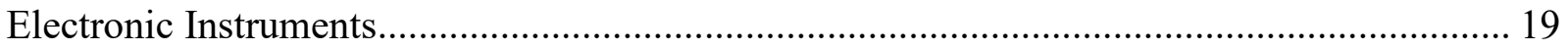

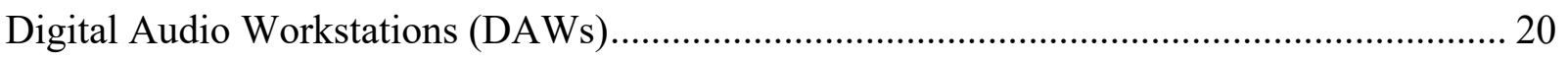

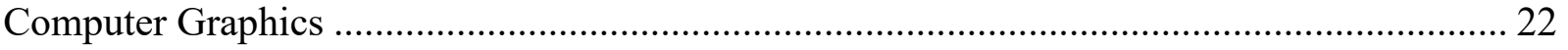

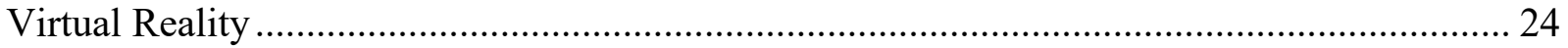

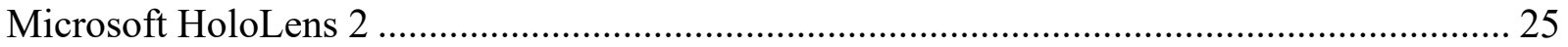

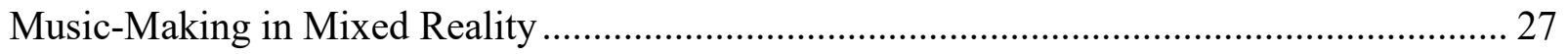

Touching Light for Percussionist and Mixed Reality Environment ……………………........ 27

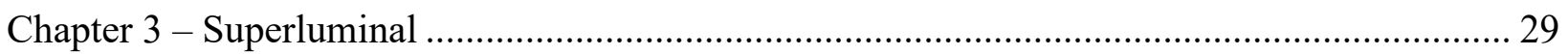

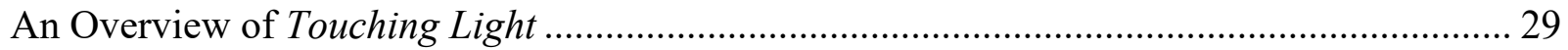

Movement 1: Simplicity …………………………………............................................ 30

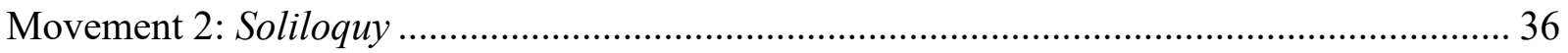

Page $\mid$ iv 


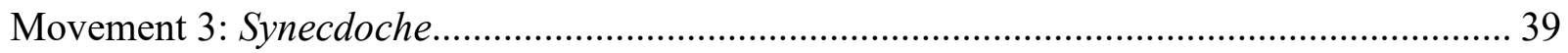

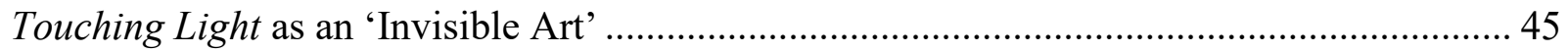

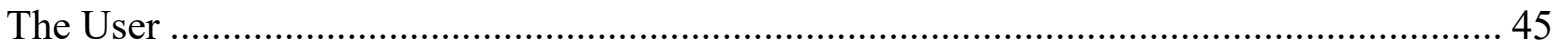

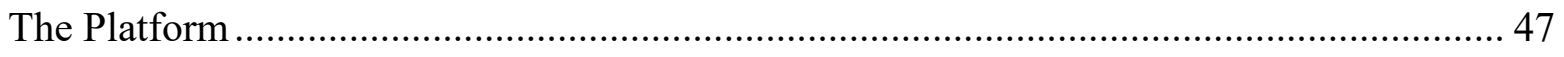

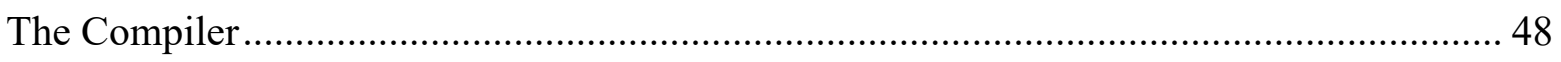

Touching Light as a 'Translucent Performance' ................................................................ 50

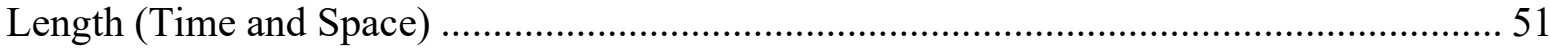

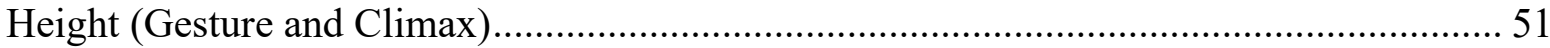

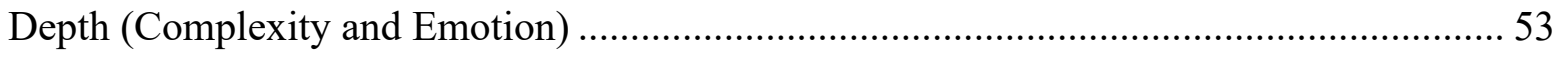

Rehearsing Touching Light (Engaging Liminalities) .................................................... 54

Tangible (Physical) Rehearsals .................................................................................. 54

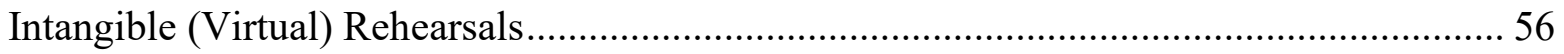

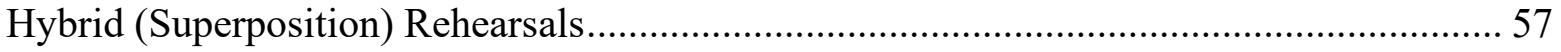

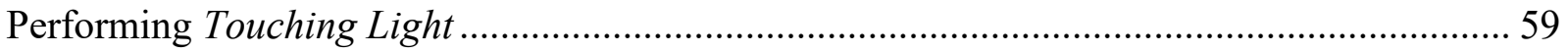

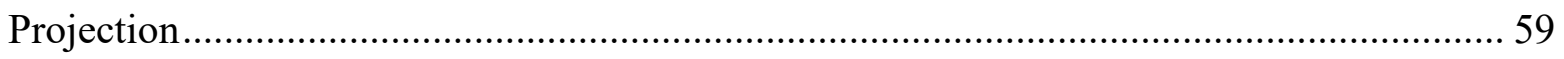

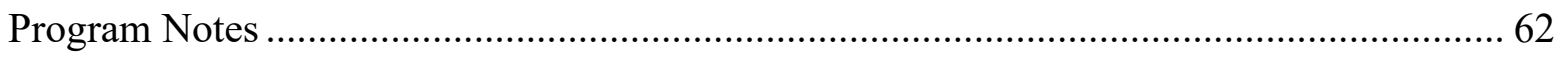

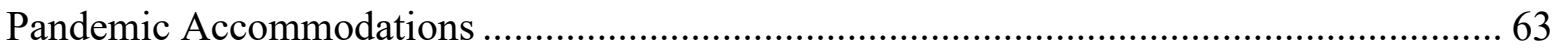

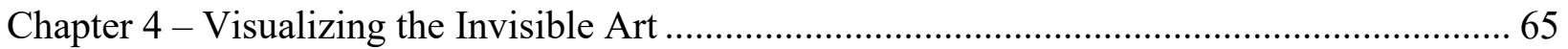

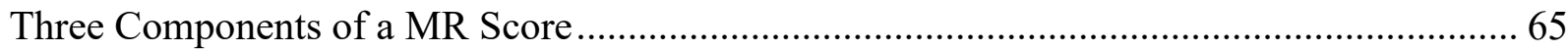

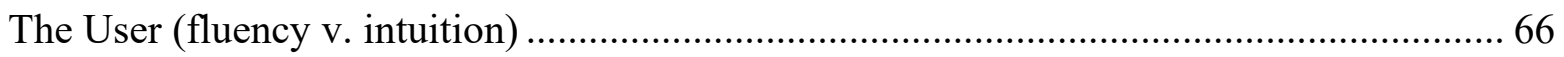

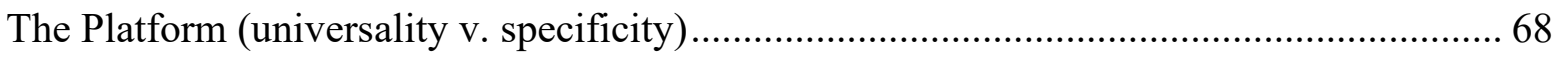

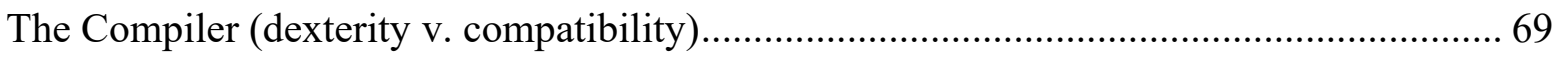

Visualizing These Component Axes ....................................................................... 71

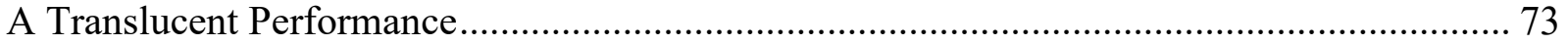

The X-axis: Length (As 'Time' and 'Size') .................................................................... 74

The Y-axis: Height (As 'Verticality’ and 'Climax') ........................................................ 77

The Z-axis: Depth (As 'Density' and 'Attention') ........................................................ 80

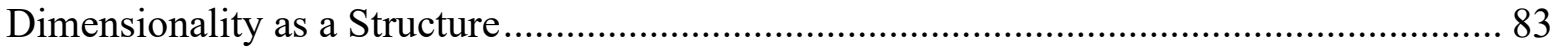

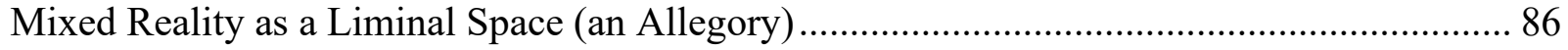

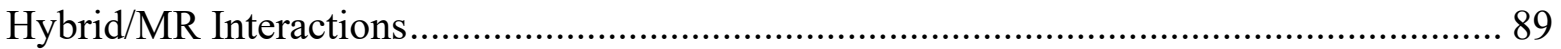

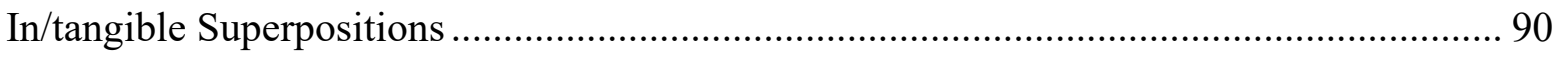

A Framework for Music-making in Mixed Reality ..................................................... 93 


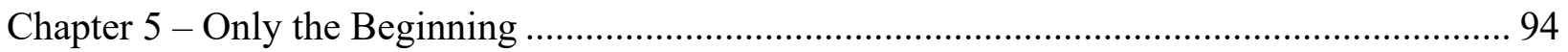

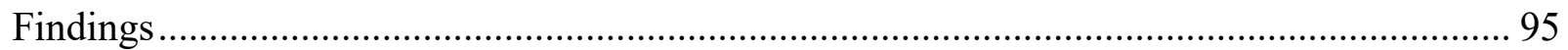

Making Music in Mixed Reality (How to Get Started, and Why You Should)....................... 96

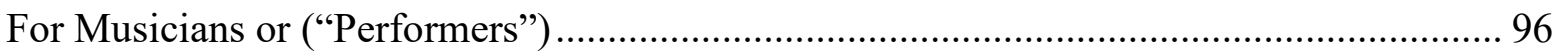

For Programmers (or "Composers") .................................................................................... 97

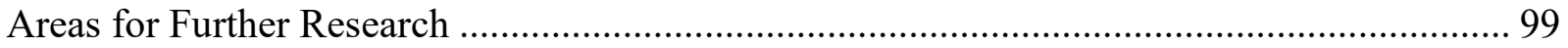

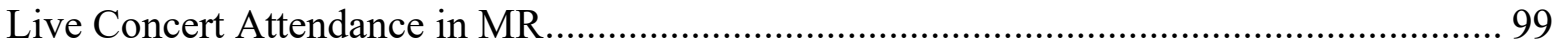

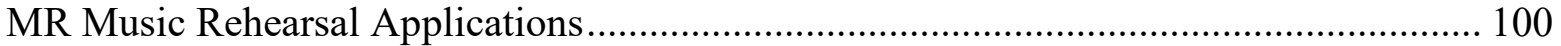

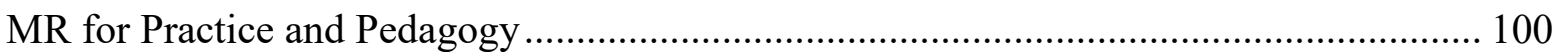

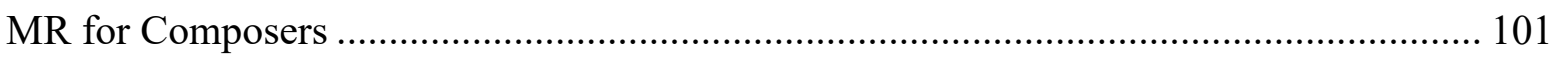

Conceptual and Philosophical Frameworks ………………………………………......... 101

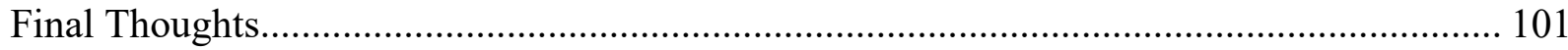

Appendix A: Program Notes for Touching Light ……………................................................ 103

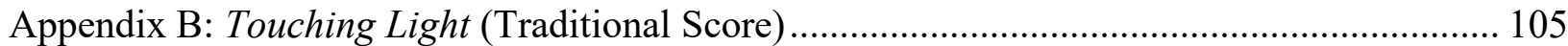

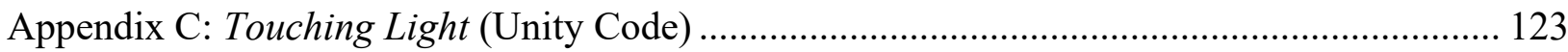

Appendix D: Touching Light 'dev-log' Blog Posts ................................................................ 130

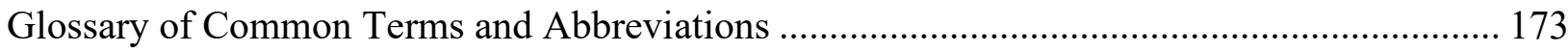

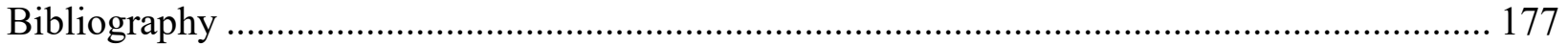




\section{List of Figures}

Figure 1: The 'piano' theme from Touching Light (i. simplicity) ................................................. 31

Figure 2: The Mixer from Touching Light (i. simplicity) ......................................................... 31

Figure 3: The five different positions (or 'scenes') from Touching Light (i. simplicity)............. 32

Figure 4: Excerpt from the score for Touching Light (i. simplicity) ........................................... 33

Figure 5: The five 'carousel images' from Touching Light (ii. soliloquy)....................................... 37

Figure 6: The first measure of Touching Light (iii. synecdoche) ……......................................... 40

Figure 7: Measures 2-3 from Touching Light (iii. synecdoche) ................................................. 41

Figure 8: Measures 4-5 from Touching Light (iii. synecdoche) ……………………………...... 42

Figure 9: The final measure of Touching Light (iii. synecdoche) …………………………........ 43

Figure 10: Diagram of Musical Form in the Touching Light Suite..............................................5 53

Figure 11: Representation of the MR environment in Touching Light (iii. synecdoche)..............60 60

Figure 12: Representation of the User's View from Touching Light (iii. synecdoche) ................. 61

Figure 13: Representation of the live feed from Touching Light (iii. synecdoche) ...................... 61

Figure 14: Representation of the Audience's View from Touching Light (iii. synecdoche) ........ 62

Figure 15: The Convergence of Touching Light in the User-Platform-Compiler Volume ........... 72

Figure 16: Representation of Near-field (blue) v. Room-scale (yellow) volumes ......................... 76

Figure 17: A Rather Small (and A Quite Large) Virtual Mixer Console ....................................... 78

Figure 18: Three Cubes from Touching Light (iii. synecdoche) ........................................... 90

Figure 19: Contextual 'hand-menu' from Touching Light (iii. synecdoche)................................ 91

Figure 20: Representation of Cubes in Motion from Touching Light (iii. synecdoche) .............. 92

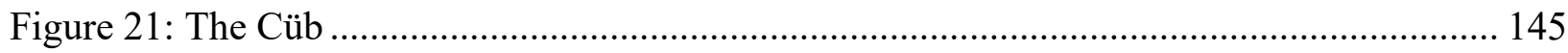

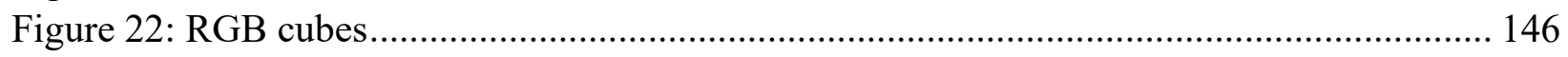

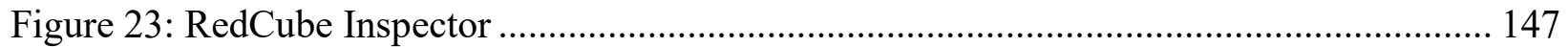

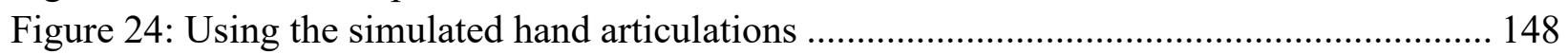

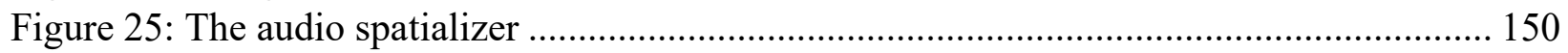

Figure 26: YouTube Video Preview - "rigidbody physics and spatialization example" ........... 152

Figure 27: It's been difficult to get the 'hand menu' to show up without hands........................ 154

Figure 28: YouTube Video Preview - "Photosphere Pre Alpha"............................................... 156

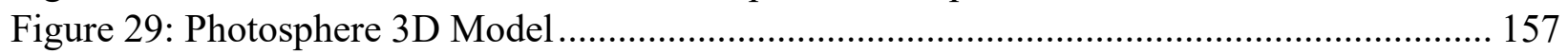

Figure 30: Photosphere Material Shader Example …………………......................................... 159

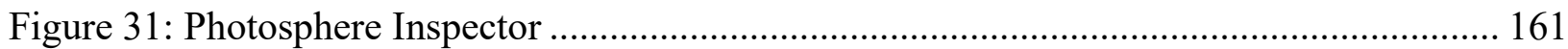

Figure 32: Photosphere HeadPositionOffset Component …………………………................... 162

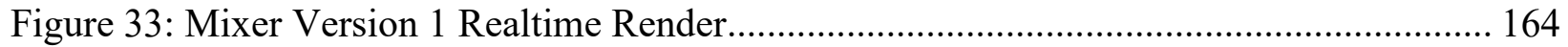

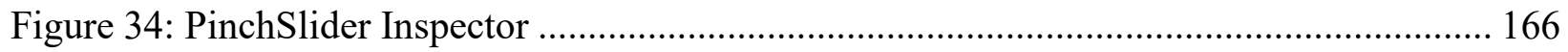

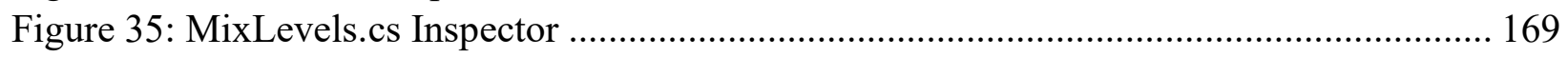

Figure 36: YouTube Video Preview - "Touching Light Mixer Show \& Tell 01" ..................... 170 


\section{List of Media Examples}

Media 1: Video demo of the first movement of Touching Light.............................35

Media 2: Video demo of the second movement of Touching Light $\ldots \ldots \ldots \ldots \ldots \ldots \ldots \ldots \ldots \ldots . \ldots . \ldots . \ldots$

Media 3: Video demo of the third and final movement of Touching Light ....................44

Page | viii 


\section{Acknowledgements}

Throughout the process of completing this document, and more broadly, this degree, I have been fortunate to have no shortage of support and assistance. This list will be necessarily incomplete, but I would like to offer my formal thanks to the following individuals.

I would first like to thank my research advisor, teacher, mentor, and friend Pf. George Willis, whose expertise and insights were invaluable in directing the breadth and depth of both this research and this degree, and moreover in encouraging me to never be satisfied with 'good enough.' It has been an honor to serve alongside you.

I would like to also thank my DMA committee, including Dr. Michael B. Vercelli, Pf. Andrea Houde, and Dr. Rhonda Reymond, whose support, direction, and affirmation throughout my time at WVU have opened my eyes to even greater possibilities.

I owe further gratitude to the faculty in WVU percussion faculty, including Pf. Willis, Dr. Vercelli, Pf. Brian Wolfe, and Pf. Mark Reilly for their encouragement during my studies, and for being sources of inspiration, as musicians, educators, and as people. I hope to emulate their example to my students and colleagues, moving forward.

I would also like to mention the sincere impact that my students and colleagues in the WVU percussion department have had on me over the past three years. It has been an honor to join you on this journey, and I look forward to all the good you will do in the future. I would like to specifically thank Ryan Jobe with whom I shared the 'grad cave' for two years for his continued friendship and camaraderie.

To my folks, I would like to offer my deepest gratitude for their unfaltering love and support.

And finally, I owe so much of this success to my lovely wife, Kristen. Thank you. 


\section{Chapter 1 - Introduction}

\section{Significance to the Field}

From the first recorded sound on April 9th, 1860, engagement with synthesized sound has continued to evolve, leading to the first radio broadcast in 1906, to the 1927 premiere of The Jazz Singer ${ }^{1}$, the first movie with sonic accompaniment. Since then, innovation in sound reproduction has only increased in frequency, feasibility, and fidelity. Leon Theremin's 1928 patent of what is commonly considered the first 'electronic instrument,' incited an everincreasing popularity for the inclusion and infiltration of electronic sounds into all musical genres. The introduction of the genre-defining like the Moog Synthesizer in 1965 and Graeme Edge's first e-drums in the 1970s has continued to deepen the synthesis of acoustic and electronic sounds. This progression of audio synthesis is paralleled by developments and innovations in visual computer graphics and virtual environments.

From their earliest inception within the realm of computer-generated imaging (CGI) in Michael Crichton's 1973 feature film Westworld ${ }^{2}$, the three-dimensional parallax developed by James Cameron for his technological groundbreaking and visually stunning Avatar $^{3}$ in 2009, or the consumer-ready, value-model virtual reality headsets like Facebook's 2019 Oculus Quest,

\footnotetext{
${ }^{1}$ The Jazz Singer, directed by Alan Crosland, featuring (Al Jolson, May McAvoy, Warner Oland, and Yossele Rosenblatt) (Warner Bros. Pictures, 1927).

${ }^{2}$ Westworld, directed by Michael Crichton, featuring (Yul Brynner, Richard Benjamin, and James Brolin) (MetroGoldwyn-Mayer, 1973).

${ }^{3}$ Avatar, directed by James Cameron, featuring (Sam Worthington, Zoe Saldana, Stephen Lang, Michelle Rodriguez, and Sigourney Weaver) (20 $0^{\text {th }}$ Century Fox, 2009).

Page | 1
} 
virtual environments have long fascinated audiences. Promising a future where the same sorts of imaginative spaces that required thousands of hours and millions of dollars to render for the theatre would be available in real-time for the consumer, Extended Reality ("XR") allows users to experience a different world, a virtual reality, where the natural laws of the physical world are not always assumed. With the development of XR platforms like the Microsoft HoloLens, the Oculus Quest, and Google Cardboard, the end-user interactions with XR environments are now widely available and continue to find applications and use-cases in many different areas of industry, lifestyle, and recreation. As the development of computer graphics and virtual environments continues to evolve, the functionality, adaptability, and accessibility of XR subsequently broadens.

Yet for as prolific and accessible as XR devices and experiences are becoming, the integration of such opportunities to live music performance is limited, particularly for environments that overlay interactable, intersectional virtual objects onto the physical world (a sub-genre known as "Mixed Reality" or "MR"). This project is positioned to uniquely combine three of our available 'virtual senses:' holographic touch, digital sound, and virtual sight, into a novel, MR musical experience. This application serves the immediate goal of integrating MR into a real-time musical performance of an original score but has further applications ranging from live performance in other mediums, musical and technological pedagogy, and as a mode to increase both musical and technological accessibility. The integration of digital technologies, particularly that of Mixed Reality, provides unique opportunities to connect with audiences and populations that would otherwise be excluded from some aspects of traditional music-making. 


\section{Limitations}

Factors that constrain the scope of this project center primarily on the focal point of the Mixed Reality application for the HoloLens and the subsequent performance of the original musical work. This project, while adaptable to other extended reality interfaces, is designed as an MR-first, HoloLens 2-first application, without implicit functionality to be deployed on other extended reality platforms. This project does not seek to provide a complete contextualization of the historical, cultural, and artistic precursors to XR technologies, mixed-instrumentation musical performances, virtual instruments, nor extended techniques for the percussionist.

Published in January of 2021, after a significant majority of the research and development of this document was completed, Luca Turchet, Rob Hamilton, and Anil Çamci's exciting project titled Music in Extended Realities ${ }^{4}$ provides insightful consideration of the state of the field of 'Musical XR ${ }^{5}$ " and includes "a comprehensive review of catalogue of published research focusing on Musical XR as documented in over 260 publications." With this combination of context and the authors' expertise in the fields of musicianship and computer science, their document also suggested a multi-faceted research agenda for the field of Musical XR. While many of these resources cited by Turchet et al. have been considered in this document (Touching Light), it seems that these projects were developed in parallel, and until recently, were unaware of each other. To that end, while every effort has been made to consider the implications of the best practices and insights presented by Turchet et al., I wish to designate a necessary limitation that, as with any new and emerging technology, many parallel and adjacent

\footnotetext{
${ }^{4}$ Turchet, "Music in Extended Realities".

${ }^{5}$ XR: "Extended Reality"

Page $\mid 3$
} 
lines of inquiry will exist simultaneously, and it is impossible, even in today's hyper-connected world, to be aware of all of them; Touching Light is no exception.

Furthermore, this document does not engage with the recent announcement of the Microsoft Mesh collaborative platform announced on March $3^{\text {rd }}, 2021^{6}$, though the author sees great potential for this new facet of MR technology in musical applications in the medium.

\section{Methodology}

In addition to engaging the available literature in this area, principal research focuses on the development of deployment architecture (an application or 'app') for the HoloLens which supports real-time, responsive interactions during a live musical performance. Research includes archival studies and masterclass observations (Microsoft HoloLens AltSpace events, Adobe MAX conference attendance, etc.) focused around computer-assisted performance, as well as Mixed Reality programming architecture and deployment, experimentation within the HoloLens development framework, and most notably, a sequential, iterative design and deployment process used to develop the HoloLens application, and a similarly iterative workflow for the composition of the original musical score. These components come together in the presentation of the functional endpoint for this project: a public performance of Touching Light for Percussionist and Mixed Reality Environment. Through a combination of practice, rehearsal, and logistical coordination, this document's suggested performance practices are applied to the preparation and presentation of Touching Light in recital. This performance exhibits functionality and opportunities that are uniquely applicable to the performance of music in Mixed Reality.

\footnotetext{
${ }^{6}$ https://www.microsoft.com/en-us/mesh
} 
The framework for the HoloLens application development cycle resembles the typical Agile development model known commonly as 'SCRUM' (as developed by Ken Schwaber in the early 1990's). The SCRUM development model consists of a series of 2-week cycles, each of which engages in the complete iterative development workflow of Planning, Design, Development, Testing, and Deployment, with a period of Review between each development cycle. While SCRUM was first designed for team-based development models, it is easily adapted in this scenario to provide consistent checkpoints and clear goals for the application development process. This project engaged in four development cycles, with each iteration of the application increasing interactivity and accessibility.

Similarly, the development of the original composition and subsequent score proceeds in tandem to the development of the HoloLens application, taking full advantage of the sorts of interactions made possible by the intersection of physical instruments and virtual environments. As the application enters its first cycle, so too have the initial sketches of the composition been developed, drawing inspiration from classical and contemporary musical styles and influences. During the middle cycles of the application development, the crux of the compositional work was completed, honing the musical direction of the work, defining its limitations, and solidifying its instrumentations and performance requirements, with clear consideration given to the interactions between the application and the score during a live performance. During the final cycle of application development, the score was rendered in its completion and engraved in a manner that is most useful to the performance of the work within a Mixed Reality environment.

Throughout the composition and development process, the author actively engaged in preparing for the public presentation of this project in the form of a performance recital, tailoring the theoretical and pedagogical frameworks developed throughout the experimentation, iteration, Page | 5 
and research phases, to the venue and audience available for the performance. A special attention was given to the balance between individual practice, in-venue rehearsal, technological robustness, and the logistical considerations, both expected and unexpected, that are necessary for a performance of a work in this medium.

Designed to facilitate the unique considerations necessary for music-making in Mixed Reality, this project centrally focuses upon a methodological approach of iterative, experiential learning. The application development process was subject to a recursive cycle of testing and deployment, tandem to the SCRUM iteration model, wherein each foundational element of the application's design is actively informed by opportunities and constraints imposed by the performance requirements for the original work. Through a continuous cycle of musical interactions, which in turn inform conceptual and revisional aspects of the application development, this work foremost serves to facilitate a musical performance of an original work designed specifically to explore this project's proposed performance practices in a public performance of Touching Light.

\section{Review of literature}

Related literature tends to fall into only a few core categorizations: those that generate foundational dialogue surrounding music technology and performance practice, those that interact and intersect with the requisite technologies that will be explored in this project, and those that consider similar scenarios in historical digital, electronic, and analog practices. While other literature may inform and shape the conception of these core areas, it is not with this literature that this project intends to engage directly.

\section{Page $\mid 6$}




\section{Principal Resources}

While resources that engage specifically with music-making in Mixed Reality are sparse, some other adjacent and related projects exist that form the foundational theoretical corpus for this research. This collection represents those resources which I will use as primary references during the principal research and development for this project. These are the seven resources which are either related to the proposed theoretical model for MR music applications, or those which serve as reference material for the technical work required to complete the project.

Joshua Barone’s “When Classical Music Meets Virtual Reality” (2019) lends a structural framework to the theoretical models used to develop the application architecture for this project. Barone's reflection of Michel van der Aa's Eight (a virtual-reality performance), and van der Aa's creative process provides further guidance for the sorts of methods and techniques that may be most beneficial and effective in the development of new Mixed Reality applications.

Similarly, Palumbo et al.'s synthesis of the theoretical models for VR environments in their 2020 article “Modular Reality: Analogues of Patching in Immersive Space," and in particular the divergence between those initial theoretical models and the practical models that the group has developed helps to define the sorts of visionary, conceptual work (both its importance and impact) that is a core part of the presuppositions supporting the development of this project's new musical architecture and performance framework. These first two principal resources provide a contextual framework for theoretical and practical considerations related to conceptualizing music-making in the blended physical and virtual volume of Mixed Reality.

In his 2020 article "Virtual 3D Environment as Composition and Performance Spaces," Marko Ciciliani considers avatar-based music-making in virtual spaces. While Ciciliani engages with a different input mechanism for the generation of musical affectation in a virtual Page $\mid 7$ 
environment, their considerations surrounding the navigation of a virtual topography directly inform this project's presentation of volumetric interactions in Mixed Reality.

\section{"Spatialized Audio in a Custom-Built OpenGL-Based Ear Training Virtual}

Environment" (Pedersen et al.) presents a technical analysis and presentation of their work in spatialized audio for XR architecture not only supports this project's assertions that access, and engagement are unique qualities of VR and MR environments, but this resource further solidifies the sorts of theoretical and conceptual models that are uniquely suited to Extended Reality applications.

The work of Serafin et al. surrounding the classification and standardization of VRMIs in their 2016 article "Virtual Reality Musical Instruments: State of the Art, Design Principles, and Future Directions" underpins the considerations of historical development as explored in this research. This document, and this group's work more broadly, has some of the most direct correlations to the current design of this research.

This trio of resources, particularly the care taken by Serafin et al. to not only catalogue but imagine the future state of the art grounds this research as innately forward-looking in its modeling. Rather than as an historical survey or a philosophical essay, this literature predicates a jumping-off point for work of this nature, as demonstrated, in different ways, by the final two principal resources from which this project will draw.

In "Holojam in Wonderland" (2018), Gochfield et al. examine the unique and meaningful ways that MR spaces can impress upon an audience a sense of engagement and interaction that may be otherwise inaccessible to audiences without a visual representation of 
sound events. The novel conditions created by their project form the basis for the conceptions of summative valuation that informs this project's foundational tenets.

Zappi et al.'s work on the Hyper Drumhead in their 2018 “Extended Playing Techniques on an Augmented Virtual Percussion Instrument" is incredibly important within the context of this project and provides a contemporary analog (2018) to the focus of this current research. While this document engages specifically with 'extended techniques' for the development of a virtual percussion instrument, the sorts of design and development considerations of that will be critical for the information of this project. Cumulatively, these seven principal resources contextualize and energize the research with which this project will engage, providing not only an historical and theoretical foundation for this effort, but simultaneously indications that this avenue of inquiry is both artistically meaningful and culturally relevant.

\section{Ancillary Resources}

This collection represents those resources which have directly informed my thinking in terms of the development of a theoretical framework for music and MR or provide foundational reference for digital music architecture and composition but are not directly referenced in the research itself. These resources can be categorized in the following manner: reviews or reports on the application of volumetric interactions (Extended Reality) to various tasks, best practices for designing assets and experiences for immersive technologies, and those which apply similar concepts will support this research, but in an exclusively virtual environment (Virtual Reality).

Work that is uniquely engaging in volumetric interactions is already sparse, in large part due to the nature of Mixed Reality as a unique method of input and interaction. Notable work on volumetrics that is ancillary to the current project include, Fusco's Voices from Beyond the Grave: Virtual Tupac's Live Performance at Coachella (2015), Graham's Environmental Page $\mid 9$ 
Histories and Personal Memory: Collaborative Works in Sonification and Virtual Reality (2017), Tatar et al.'s Respire: Virtual Reality Art with Musical Agent Guided by Respiratory Interaction (2019), and Wilson et al.'s Sound, Space, Image and Music: Hybridity in Creative Process through Technology, Interactivity and Collaboration (2012).

On the topic of best practices, informative resources include Bouënard et al.'s Hybrid Inverse Motion Control for Virtual Characters Interacting with Sound Synthesis (2012), GalletBlanchard's VR "Montmartre in the Jazz Age": The Problematics of Virtual Reality in Researching and Teaching Multicultural History (2005), Pressing's Some Perspectives on Performed Sound and Music in Virtual Environments (1997), Rabeler's A Spatial Color-Sound Model for Mixed Reality (2011), Çamc1 et al.'s Audio-First VR: New Perspectives on Musical Experiences in Virtual Environments (2020), and Serafin et al.'s Reflections from Five Years of Sonic Interactions in Virtual Environments Workshops (2020).

Inclusive are also projects that consider uniquely Virtual-Reality experiences, including Dingman's Rock Band VR: Rock Band's Roaring PC Debut Showcases Oculus Touch's Potential (2017), Hamilton's Be a Rock Legend in Your Living Room (No Guitar Required) (2003), Kenwright's There's More to Sound Than Meets the Ear: Sound in Interactive Environments (2020), Lombardo et al.'s A Virtual-Reality Reconstruction of Poème Électronique Based on Philological Research (2009), Lucas et al.'s To a Cultural Perspective of Mixed Reality Events: A Case Study of Event Overflow in Operas and Concerts in Mixed Reality (2012), Rasika et al.'s Narrowcasting and Multipresence for Music Auditioning and Conferencing in Social Cyberworlds (2015), and Wang et al.'s Virtual and Real-Time Synchronous Interaction for Playing Table Tennis with Holograms in Mixed Reality (2020). 


\section{Related Resources}

This collection represents those resources that, while related to the topic to varying degrees, are outside of the scope of the goals of this project. This includes references on the history and development of computer music, extended reality, etc., as well as resources that explore parallel work in other artistic and scientific fields. These resources include, but are not limited to:

- Baran et al.'s Interdisciplinary Concepts for Design and Implementation of Mixed Reality Interactive Neurorehabilitation Systems for Stroke (2015)

- Chang et al.'s EEG Analysis of Mixed-reality Music Rehabilitation System for Post-stroke Lower Limb Therapy (2019)

- Wang et al.'s The Framework of Simulation Teaching System for Sports Dance Based on Virtual Reality Technology (2017)

- Channel at al.'s Creating Virtual Internships in the Music Business (2010)

- Chirico et al.'s Virtual Reality and Music Therapy as Distraction Interventions to Alleviate Anxiety and Improve Mood States in Breast Cancer Patients during Chemotherapy (2020)

- Gaugne et al.'s EvoluSon: Walking through an Interactive History of Music (2017)

- Hass' Introduction to Computer Music (2020)

- Harmon's Diffusion of Virtual Reality in Audiences Viewing Popular Music (2018)

- Hugill's Internet Music: An Introduction (2005)

- Innocenti et al.'s Mobile Virtual Reality for Musical Genre Learning in Primary Education (2019)

- Litterst's Random Recess: Music Teachers In The Twilight Zone (2017) 
- McGrath et al.'s The User Experience of Mobile Music Making: An Ethnographic Exploration of Music Production and Performance in Practice (2017)

- Miranda's Introduction: Leonardo Music Journal 29 (2019)

- Peire Serrate's Volume I: Music in Virtual Reality: Musical Immersivity and Interactivity and Volume II: Meeting (1.0, 2.0 and 3.0) and Hillary (Concert and VR) (2019)

- Rocchesso et al.'s “Interaction by Ear.” International Journal of Human-Computer Studies (2019)

- Ward's Technology and the Transmission of Tradition: An Exploration of the Virtual Pedagogies in the Online Academy of Irish Music (2019)

While each of these resources are related to Extended Reality conceptions in some way, their focus is divergent from the goals of this project, and therefore will not factor into the theoretical or practical elements presented herein.

\section{Overview of This Document}

In Chapter 2, 'From Then, 'til Now,' I will explore the historical development of music technologies, tracing the ways in which each of these innovations opens our eyes to an otherwise unseen history of Mixed Reality as the promised culmination of 'imagination made manifest.' With this foundation, we may begin to consider that Mixed Reality as a musical and performative medium is the natural outgrowth of decades, if not centuries of technological innovation capturing the imaginations of artists and audiences alike. MR is not the first $^{7}$, nor will

\footnotetext{
${ }^{7}$ Beethoven composed Wellington's Victory for mechanical orchestra in 1813...! 7 "Wellingtons Sieg, Op.91

(Beethoven, Ludwig van) - IMSLP: Free Sheet Music PDF Download.” Accessed February 3, 2021.

https://imslp.org/wiki/Wellingtons_Sieg,_Op.91_(Beethoven,_Ludwig_van).

Page $\mid 12$
} 
it be the last, effort to combine our virtual and physical perceptions into a singular artistic canvas. Within a Mixed Reality environment, music-making in both physical and virtual volumes intersect and inform one another; the percussionist's unique training in navigating threedimensional space provides a natural affinity for mixed-reality music-making.

'Superluminal,' the third chapter of this document will present an account of the development of Touching Light, the original musical work created in conjunction with this document, through the three principal frameworks presented in herein. I will explore the specific combination of user, platform, and compiler featured in Touching Light, and by defining this specific case, may more fully understand the importance of making these decisions early. In exploring the ways that Touching Light visualizes the 'invisible art,' I will examine how both the logistical and conceptual considerations of dimensionality serve to deepen the musical intentionality of the performer. Finally, by examining the liminalities engaged in Touching Light, I will explore the ways in which an understanding of liminal spaces allows the performer to more fully engage in the MR music-making process.

Having recognized an historical precedent for Mixed Reality music-making, I will then explore how the conceptual models introduced provide a methodological framework by which to design generalized Mixed Reality experiences in Chapter 4, 'Visualizing the Invisible Art.' My first consideration will be to delineate the three core components of any Mixed Reality score: the user, the platform, and compiler. Each of the elements require specific care and consideration as they will form the foundational framework within which the musical medium will subsist. While all manner of variations of these three core components may exist, I conclude that prioritization of intuition, universality, and compatibility must be measured against the benefits of fluency, specificity, and dexterity in each of those core components. 
In my examination of 'Touching Light as a Translucent Performance,' I will present six ways in which volumetric practices already inherent to the percussionist's physical artistry inform, and may be adapted to, musical performance in a Mixed Reality environment. In considering the three principal coordinate axes of length, height, and depth, in conjunction with the broader philosophical considerations that each of these dimensions provide, I begin to formulate a series of questions that will serve as helpful precursors to our engagement with MR as a musical medium.

Finally, in considering the rehearsal process for Touching Light, I will describe inherent boundary zones, or 'liminal spaces,' that are present in the Mixed Reality between physical and virtual environments. By conceptualizing these liminal regions as a sort of permeable interface between physical and virtual environments, objects, and interactions, I will begin to more deeply consider the sorts of unique hybrid experiences that are available to those artists and musicians who make music in Mixed Reality.

The final chapter of this document is reserved for conclusions, findings, and no shortage of surprises encountered throughout the process of developing this document. This eighth chapter will also offer some concise thoughts surrounding the benefits of performing music in Mixed Reality and important considerations as we look toward the future of the medium, both as a space for innovation, as well as at possible ways that it may be adapted to existing the forthcoming literature that is not necessarily written for Mixed Reality. 


\section{Chapter 2 - From Then, ‘til Now}

Mixed Reality as the Historical Culmination of Technological Imagination

As of January $5^{\text {th }} 2020$, the Oxford English Dictionary defines 'technology' to mean, "the branch of knowledge dealing with the mechanical arts and applied sciences; the study of this," 8 citing that previous definitions had become 'obsolete' - certainly with the wide variety of modern sentiments toward 'technology,' from complete acceptance to absolute rejection, 'technology' has taken on a life of its own, no longer a finite collection but rather an evergrowing category... yet 'technology', for all of its new meaning, the term once described 'a discourse or treatise on an art. ${ }^{9}$ And so, perhaps it is not so profound to suggest, that this discourse in art generally, and music specifically, it is not so new, is not an invasive rebuttal of that which came before, but a synthesis, a discourse, of all that the art contains.

\footnotetext{
8 “'Technology, n.” In OED Online. Oxford University Press. Accessed January 5, 2021.

http://www.oed.com/view/Entry/198469.

1. A discourse or treatise on an art or arts; esp. (in later use) a treatise on a practical art or craft. Obsolete.

In quot. 1612 perhaps: academic discussion or disputation generally.

1612 tr. I. Casaubon Answere Epist. Peron sig. A3v Men, void of Gods spirit, commonly and promiscuously did dispute of spirituall things, and conuert Theologie into technology, that is, make no other vse of Diuinity but as a matter of learned, or artificiall discourse, as they talke of other arts and sciences out of humane reason.

1615 G. Buck Third Universitie of Eng. xlviii, in E. Howes Stow's Annales (new ed.) 988/2 An apt close of this general Technologie.

1628 T. Venner Baths of Bathe 9 Heere I cannot but lay open Baths Technologie.

1706 Phillips's New World of Words (new ed.) Technology, a Description of Arts, especially the Mechanical.

1860 Vanity Fair (N.Y.) 7 Apr. 235/1 We have Classical Dictionaries, Dictionaries of Science,..Cyclopædias and Technologies without Number.

9 Ibid. 
This chapter will simultaneously consider the parallel histories of music and technology and begin to propose the idea that while 'Mixed Reality' has a specific meaning in $21^{\text {st }}$-century technology spaces, the concepts that drive innovation and inspire those working in this area are synonymous with those things that musicians and artists have been imagining throughout history: through music, providing some sort of window into the ineffable. My proposal in this chapter is that MR as a technology is the natural progression of those synonymous desires. In order to contextualize the integration of technology and artistry, of musician and machine, let us consider the rich heritage of this harmonization of tangible and intangible, of physical and virtual, of perception and reality.

\section{Sound Recording and Reproduction}

April $9^{\text {th }}, 1860$ is often cited as the earliest instance of 'recorded music;' it is on this date that Édouard-Léon Scott de Martinville captured a recording of the French folk song, $\mathrm{Au}$ Clair de la Lune. ${ }^{10}$ While it is certainly the case that music technology had been permitting the recording of music in the form of notation for some time before Scott's phonautograph, the invention of this device would fundamentally alter the ways that audiences would engage with music-making. With the allowance for 'captured sound,' it is no surprise that the imaginations of artists and inventors alike would give birth to the development and commercialization of Thomas Edison's phonograph cylinder in $1877,{ }^{11}$ providing a way to not only capture recorded sound, but to reproduce it. With a world of sound at our fingertips, years' worth of music in our pockets, it is

\footnotetext{
10 "First Sounds". March 27, 2008. Accessed January 5, 2021. https://www.firstsounds.org.

${ }^{11}$ Edison, Thomas. "Patent for Phonograph Recorder.” Patent 42249 Summary. Industry Canada, June 15, 2015. https://www.ic.gc.ca/opic-cipo/cpd/eng/patent/42249/summary.html.
} 
easy to forget that for most of human history, music-making required a live musician; for as accessible as music recordings are in the twenty-first century, that there is still value placed on music-making, there are still garage bands, and symphony orchestras; recordings and reproductions are not enough. Yet there is no denying that the 'democratization of sounds, ${ }^{12}$ with its roots in Edison's 1877 invention, would continue to blur the lines between physical and virtual in ways that the $19^{\text {th }}$-century luminary could never have imagined.

\section{Radio}

Soon after the commercialization of 'recorded sound' via the 1887 Berliner Gramophone, telegraphy techniques that had been proposed as early as 1880 by American inventor David Edward Hughes, German physicist Heinrich Rudolph Hertz would conclusively prove the possibility of transmitting airborne electromagnetic waves in 1888, building on the work of Scottish mathematical physicist James Clerk Maxwell's early work with electromagnetism. Hertz's proof and subsequent experimentation ${ }^{13}$ would directly influence the development of radio broadcast technologies near the turn of the century. From the first wireless transmission of the human voice in 1900, to among the first radio sports broadcast, coverage of a West Virginia Mountaineers v. Pittsburgh Panthers college football game in 1921, the development and understanding of radio and related technologies would once again illuminate the imaginations of audiences and artists alike - the virtual world of the human mind, complete with its ability to

\footnotetext{
${ }^{12}$ Ideas. “How Design Is Helping to Democratize Music Making | Adobe XD Ideas.” Accessed January 5, 2021. https://xd.adobe.com/ideas/perspectives/social-impact/how-design-helps-democratize-music-making/.

${ }^{13}$ Hertz, Heinrich, and William Thomson Baron Kelvin. Electric Waves: Being Researches on the Propagation of Electric Action with Finite Velocity Through Space. Macmillan, 1893. 
overlay the physical sensation of listening to music with all manner of affectation and visualization, once more mixed the realities of the audience and the performer, lessening the distance between these two artistic spaces and increasing accessibility in profound and innumerable ways.

\section{Synthesized Audio and Music Visualization}

As reproductions of recorded audio continued to proliferate the shifting market for consumer music, so did the understanding of sound production and synthesis. Many technological developments contributed to the unfolding of sonic synthesis ${ }^{14}$, but as can be said of many increases in understanding related to first principles, the more complete the perspective, and the broader the access to the core conception of a thing, the more fully it can be understood, and in many cases, the more discretely it can be manipulated. Sound production had, for centuries, before, been a primarily aural and kinesthetic practice: a musician would audiate the sound, the timbre, the tone, the intonation that they desired to produce, and then through practiced motion, whether by vocalization or engaging an implement upon an instrument, attempt to match their desired, albeit imagined sound, with their physical instrument.

Yet as technological understanding of electromagnetic oscillation, and the connection of its measured frequency, amplitude, distortion, etc. to the fundamental waveforms that seem commonplace in a modern understanding of sound production, it was The Atari Video Music visualizer in $1976^{15}$ that would translate the electromagnetic frequency data present in analog

\footnotetext{
${ }^{14}$ This terminology may seem redundant, but here is used to describe the specific focus of early electronic musicians and sound engineers toward developing a visual realization of sonic waveforms, e.g., oscilloscopes, etc.

${ }^{15}$ Nielsen Business Media, Inc. “Tape/Audio/Video”. 
audio signals of the late $20^{\text {th }}$ century into a systematically designed audio visualization engine, marrying the aural experience with the visual, fundamentally enabling consumer audiences to 'see music.'

\section{Electronic Instruments}

With access to consumer computer technology on the rise in 1970's and 80's and a growing interest in digital and electronic music-making, the inevitability of 'electronic instruments' seemed inevitable ${ }^{16}$. While electrified instruments were being developed in parallel, ${ }^{17}$ it was Lev Sergeyevich Termen (also known as: Léon Theremin) 1926 'Theremin' that would constitute the first 'electronic instrument,' producing sound via electronic circuitry.

It was not until 1964, however, that the developments in mixing physical and virtual realities would leap forward yet again with the release of Robert "Bob" Moog's 'Moog Synthesizer.' Bob's father, one of the first amateur radio operators in the United States ${ }^{18}$, was an engineer whose basement workshop served as his son's sanctuary from the more traditional

\footnotetext{
${ }^{16}$ An electronic instrument is unique from an 'electric' or 'electrified' instrument in a few principal ways: firstly, while an electronic instrument certainly requires electricity to function, unlike an electrified instrument, an electronic instrument has no direct amplification of an acoustic signal as part of its core design. The electric guitar, for instance, invented in 1932, using the flux generated by electric 'pickups' to amplify the acoustic tone of the instrument. An electronic guitar (e.g., Magic Instruments 'Mi Guitar'), in contrast, produces sounds that are completely synthetic. (“Even N00bs Can Rock Out on Magic Instruments’ New Guitar | WIRED.” Accessed January 11, 2021. https://www.wired.com/2016/04/even-n00bs-can-rock-magic-instruments-new-guitar/.)

${ }^{17}$ Hempstead, et al., Encyclopedia of 20th-Century Technology, p. 793.

${ }^{18}$ Paul Théberge, “Any Sound You Can Imagine”.

Page $\mid 19$
} 
musical endeavors that his mother insisted on ${ }^{19}$. It was here that the seeds of what would historically become the first commercially successful synthesizer were sown, a realization, a synthesis of Moog's imagination in practice, his physical piano practice through a virtual instrument, a mixing of realities.

The Moog Synthesizer would be the first of many electronic instruments, including synthesizers of all shapes and sizes, alongside the electronic reimagining of all sorts of musicmaking devices, both real and imagined. With an understanding of the fundamental components of a digital audio signal garnered from decades of experimentation with radio and other electromagnetic sound technologies, the electronic, now 'virtual' instruments, provide access to a multitude of life-like recreation of physical instruments, as well as a near-limitless potential for the generation of new, unique sounds.

\section{Digital Audio Workstations (DAWs)}

It is a particular sort of joy to hear something new for the first time; as we age, many of us are constantly surrounded by sound, and eventually, it can seem like we've 'heard it all.' Yes, perhaps there is still some unrealized combination of recognizable sounds, perhaps there is some slight variation in timbre, a bridge further to our emotional experience, but it is much less common for an adult to experience a uniquely 'new' sound. So much is this the case that entire musical genres have developed around the recontextualization of non-music sounds within a musical context, seeking that single percentage point to broaden the musical palette.

\footnotetext{
19 Trevor Pinch and Frank Trocco, Analog Days.
} 
Yet like taking the blinders off, or stepping out of Plato's proverbial cave, sound synthesis technologies, and in particular, digital audio workstations place before us a sort of Frankenstein scenario: with complete control of sonic granularity at our fingertips, it is possible to create sounds, and perhaps by extension, music, that has never been heard before. While the first digital audio workstation was developed by Soundstream, Inc., a US-based digital audio recording company in $1978^{20}$, the early 1990 's saw the development of DAWs which are still industry standards in the early $21^{\text {st }}$-century: Digidesign's ProTools from 1991, and Steinberg's Cubase from 1996.

The development of DAWs provided a level of amateur accessibility (and by extension, more common understanding) of the craft honed by sound engineers in the decades preceding their proliferation. At the time, DAWs provided the ultimate mixing of physical and virtual music-making, allowing a level of granular control that was thus far unprecedented in a virtual format. Early iterations built to emulate the analog mixing workstations after which they were modeled, today, the development of Virtual Sound Technology Instruments (VSTs; completely digital synthesizers with a variety of forms and functions) allow DAW users to create entirely virtual symphonies with little more than their mouse and keyboard.

And, just like the development of an instrument, the level of fidelity, nuance, and emotional expression that is possible with these VSTs is becoming increasingly mature; in a video blog post from May 2020, prominent electronic musician and sound engineer Andrew Huang considers that "This [referring to a physical, Fender 'American Ultra Jazz Master' electric guitar] is a preset..." conceding subsequently that he is "expecting a little bit [emphasis mine] of

\footnotetext{
${ }^{20}$ Kefauver, et al., Fundamentals of Digital Audio.
} 
pushback for that one, in the comments" ${ }^{21}$. But his point is taken. In many traditional music circles, the idea of 'digital instruments' is met with some resistance, often ranging from trepidation and competition to distaste and dismissal, an affront to value of both specificallyhuman and specifically-acoustic music-making. And while there is no shortage of studies that suggest that music-making is an inherent human experience ${ }^{22}$, how long before these synthesized instruments become indistinguishable from acoustic ones, how long before they are just as expressive? Or even more expressive? Already we see an increase in digital music-making and digital music sales year-over-year ${ }^{23}$; yet another 'mixed reality.'

\section{Computer Graphics}

Not unlike the trajectory of growth experienced by digital audio throughout the twentieth century, the development of computer graphics, and specifically the ability for a scientist or engineer to render three-dimensional objects using a two-dimensional interface would radically change the sorts of visualizations that audiences would come to expect in popular media ${ }^{24}$. Fundamentally, CGI promised that, given time, advancements in the technology would render computer graphics indistinguishable from traditionally captured media, a promise of a mixed visual reality, seamlessly rendering the impossible worlds of our imaginations. In 2020, computer graphics have improved to such a degree that full-body replacements of deceased

\footnotetext{
${ }^{21}$ Huang, Andrew. This Was a Total Gamechanger for My Sound Design! YouTube Video, 2020. https://www.youtube.com/watch?v=GXQHiozYGTE.

${ }^{22}$ Schulkin, et al., "The Evolution of Music and Human Social Capability”.

${ }^{23}$ Joshua P. Friedlander, "Mid-Year 2019 RIAA Music Revenues Report," 3.

24 “The Computer Graphics Book Of Knowledge.” Accessed January 13, 2021. https://www.cs.cmu.edu/ ph/nyit/masson/history.htm.
} 
actors have been employed in Hollywood feature films, both in genres that one might expect such a technology may exist (e.g., Carrie Fisher likeness as General Leia Organa in Rian Johnson's 2017 Star Wars: The Last Jedi ${ }^{2526}$ ), as well as those where no such 'science fiction' would be expected (e.g., James Dean's likeness in Anton Ernst's forthcoming Finding Jack ${ }^{27}$ ).

Vital to the strategies of dozens of industries in the $21^{\text {st }}$-century, CGI is baked into much of modern cinema and television, not to mention the underpinning of gaming spaces, particularly for PC and console gamers. Yet far beyond simple entertainment, computer graphics research powers the device that allows you to read this sentence, as well as the device that allowed me to type it - the use of technology, when deployed elegantly, expertly, and with purpose expands our reality, while invariably mixing it with a virtual one, a world beyond what we can see, and ignites our imagination in the process, whether we desire to see different worlds, or simply words on a screen. Invariably, in our visual entertainment, we have come to depend on a mix of realities.

${ }^{25}$ Zakarin, Jordan. "It Took More CGI than You Think to Bring Carrie Fisher into The Rise of Skywalker.” SYFY WIRE, January 7, 2020. https://www.syfy.com/syfywire/it-took-more-cgi-than-you-think-to-bring-carrie-fisher-intothe-rise-of-skywalker.

${ }^{26}$ Star Wars: The Last Jedi, directed by Rian Johnson, featuring (Mark Hamill, Carrie Fisher, Adam Driver, Daisy Ridley, John Boyega, Oscar Isaac, Andy Serkis, Lupita Nyong'o, Domhnall Gleeson, Anthony Daniels, Gwendoline Christie, Kelly Marie Tran, Laura Dern, Frank Oz, and Benicio del Toro) (Walt Disney Studios Motion Pictures, 2017).

${ }^{27}$ Holson, Laura M. “A C.G.I. James Dean? Some in Hollywood See ‘an Awful Precedent' (Published 2019).” The New York Times, November 8, 2019, sec. Arts. https://www.nytimes.com/2019/11/07/arts/james-dean-cgimovie.html. 


\section{Virtual Reality}

So perhaps it is not so great a leap to approach an understanding of the draw of virtual spaces as a replacement for physical ones. Stemming from the first computer graphics experiments in the 1950's and accelerated by the first representations of 3-D CGI in film in Richard T. Heffron's 1976 Futureworld ${ }^{28}$, just a year before the famous Death Star simulation in George Lucas' 1977 Star Wars (later, Star Wars: A New Hope ${ }^{29}$ ), the three decades preceding the turn of the millennium saw the origins of many of our most recognizable virtual reality imaginings in film (i.e., Steven Lisberger's 1982 Tron $^{30}$, John Badham's 1983 WarGames ${ }^{31}$, and the Wachowski’s 1999 The Matrix ${ }^{32}$ ) and literature (i.e., William Gibson’s 1984 novel Neuromancer ${ }^{33}$, and Orson Scott Card's 1986 Ender's Game $^{34}$ ).

As CGI continued to develop in sophistication, it is not wholly surprising that the promise of interacting with a virtual world would precipitate the development of the earliest

\footnotetext{
${ }^{28}$ Futureworld, directed by Richard T. Heffron, featuring (Peter Fonda, Blythe Danner, Arthur Hill, Stuart Margolin, John Ryan, and Yul Brynner) (American International Pictures, 1976).

${ }^{29}$ Star Wars, directed by George Lucas, featuring (Mark Hamill, Harrison Ford, Carrie Fisher, Peter Cushing, and Alec Guinness) (20 th Century Fox, 1977).

${ }^{30}$ Tron, directed by Steven Linsberger, featuring (Jeff Bridges, Bruce Boxleitner, David Warner, Cindy Morgan, and Barnard Hughes) (Buena Vista Distribution, 1982).

${ }^{31}$ WarGames, directed by John Badham, featuring (Matthew Broderick, Dabney Coleman, John Wood, and Ally Sheedy) (MGM/UA Entertainment Company, 1983).

${ }^{32}$ The Matrix, directed by The Wachowskis, featuring (Keanu Reeves, Laurence Fishburne, Carrie-Anne Moss, Hugo Weaving, Joe Pantoliano) (Village Roadshow Pictures, 1999).

${ }^{33}$ Neuromancer, William Gibson (New York: Berkley Publishing Group, 1984).

${ }^{34}$ Ender's Game, Orson Scott Card (New York: Tor, 1985).
}

Page $\mid 24$ 
forays into commercially available virtual reality devices, like the Forte VFX1. Simple by modern standards, the VFX1 provided a level of audio and visual immersion in a virtual space that was previously impossible and, if the proliferation of VR headsets on the market in 2020 is any indication, would anticipate a popularization of then unexplored method of mixing realities: through total sensory immersion.

\section{Microsoft HoloLens 2}

In February of 2016, Alex Kipman and Kudo Tsunodathe, the original design team for the first Microsoft HoloLens invited their audience to "...together, transform the world, through holograms." ${ }^{35}$ While the degree to which the world has 'changed' due to the introduction of Microsoft's headwear is still being realized, the Microsoft HoloLens, and its successor, the Microsoft HoloLens 2 ("HL2"), provide an important alteration to the traditional VR formula, an medium that Microsoft refers to as 'Mixed Reality' ("MR").

Whereas in VR the world around you is shut out and replaced by a completely computergenerated world, MR suggests that there are still important things that the user might want to be aware of in the world around you, and furthermore, that it may be beneficial if the virtual experience is not agnostic of the physical environment within which the user exists. To that end, the HL2 was developed with a combination of groundbreaking technologies connecting in unprecedented ways to make good on the promise of Tony Stark's J.A.R.V.I.S. ${ }^{36}$ artificial

\footnotetext{
${ }^{35}$ Microsoft HoloLens. Microsoft HoloLens: Welcome to the Team, 2016. https://www.youtube.com/watch?v=C98qaPAMVQU.

${ }^{36}$ Iron Man, directed by Jon Favreau, featuring (Robert Downey Jr., Terrence Howard, Jeff Bridges, Shaun Toub, and Gwyneth Paltrow) (Paramount Pictures, 2008).

Page $\mid 25$
} 
intelligence or the U.S.S. Enterprise's ${ }^{37}$ holodeck: virtual objects that respond to physical interactions and engage with a physical environment.

Microsoft's HoloLens technology synthesizes three foundational technologies to allow for the seamless integration of physical and virtual spaces. Moving outward from the user, the HoloLens features hardware and software that are user-facing; these functions allow the HoloLens to determine the optimal calibration of the dual-screens in the 'lens,' but also permit the device to track the gaze of the user, permitting gaze-based interactions as an additional control scheme. Secondarily is the 'lens' itself, which consists of a pair of specially designed screens that facilitate the projection of the generated virtual overlay of holographic objects. Not unlike the technologies involved in 3D movies, the HoloLens projects two slightly different holograms, one for each eye, the combination of which allows for objects that are projected onto the 2D lens to appear in 3D space for the user. Finally, and what sets the HoloLens apart from a high-end VR headset, is the sophisticated components that are outward-, or 'environmentfacing,' including ambient light sensors, HD cameras, and four proprietary 'environmental awareness sensors' that allow the device to map the physical surfaces of the environment around it and generate a sort of 'mesh' that it uses to determine what is open-space and what is a solid object. This data is processed in real-time to allow the user to interact with the projected holographic objects using touch, voice, and gaze, and to allow the HoloLens to determine where those objects may exist and where they cannot.

\footnotetext{
${ }^{37}$ Star Trek: The Next Generation, directed by Gene Rodenberry, featuring (Patrick Stewart, Jonathan Frakes, LeVar Burton, Denise Crosby, Michael Dorn, Gates McFadden, Marina Sirtis, Brent Spiner, and Wil Wheaton) (Paramount Domestic Television, 1987-2006).
}

Page $\mid 26$ 
This environmental awareness provides a level of connectivity between the user and the environment that has been previously impossible due to the design of traditional VR technologies, and it is precisely this volumetric synthesis, this true mixing of realities that presents such a rich environment for artistic opportunities that engage both virtual and physical spaces.

\section{Music-Making in Mixed Reality}

With the release of the HoloLens 2, a consumer-grade Mixed Reality interface is finally available. Because of the platform's unique ability to provide access to the shared space between the physical world and the virtual world (i.e., a 'Mixed Reality volume'), artists, musicians, and other creatives may now, more than ever, begin exploring, developing, and performing in this transitional, 'liminal' space, this newly accessible shared environment. In the following chapters, I will explore three fundamental ways to engage with Mixed Reality as a creative medium: through the transcription of physical interactions into a Mixed Reality environment, through translation of compositional intention and conceptual realization for works for Mixed Reality performer, and through the transformation of the ways that we engage gesture and volumetrics to facilitate musical experiences. In all these areas, music-making in Mixed Reality is both uniquely suited to the sorts of interactions and physical gestures already employed by classically trained percussionists, as well as the exciting opportunities that music-making in Mixed Reality offers to broaden and deepen our physical experience of musical performance through its conjunction with a virtual one.

\section{Touching Light for Percussionist and Mixed Reality Environment}

Connected to the theoretical and philosophical considerations present in this research is the hands-on application of this proposed framework through the preparation of a work for Page $\mid 27$ 
percussionist and Mixed Reality environment that I have developed called Touching Light. Touching Light is designed to be an instructional experience for the performer/user, facilitating a meaningful engagement with the sorts of artistic possibilities that are exclusive to music-making in Mixed Reality, a vehicle to explain, explore, and exhibit both the functionality and potentiality of Mixed Reality as a medium that is equally accessible to audiences with or without expert knowledge of the fields of MR technology or music performance, and finally as a meaningful musical work in and of itself that exists not only to serve the framework presented in this document, but rather as the natural outgrowth, the functional endpoint of the framework itself.

Throughout, concrete examples of how best practices, conceptual models, and methodologies might be implemented will corroborate the philosophical and theoretical discussions of the same. 


\section{Chapter 3 - Superluminal}

Composing and Performing Touching Light

This chapter directly discusses the development of Touching Light, an original work for percussionist and Mixed Reality environment, considering composition, construction, rehearsal, and performance as the core elements of this work's creative process. The beginning of this chapter will provide an overview of the traditional score for Touching Light, highlighting the compositional decisions involved. The methodological framework presented in this document is then highlighted throughout the development process, as Touching Light is philosophically and artistically concurrent to the theories presented as part of this document.

\section{An Overview of Touching Light}

Touching Light for Percussionist and Mixed Reality Environment is an original composition created in conjunction with this research. Intended to explore the unique opportunities that Mixed Reality offers a performer musician, Touching Light is scored for the Microsoft HoloLens 2 and percussion instruments. The composition exists as a suite in three parts, each of which feature a different function of MR for the performer to interact with. Each of the three movements connect a musical or conceptual 'focus' to a related type of MR interaction with the intention of grounding each of these interactions within the context of three definitions: simplicity, soliloquy, and synecdoche. These three words serve as both the titles of each of the three movements, as well as the coordinating element between the traditional musical and MR elements. The physical instrumentation of each movement is left to the discretion of the performer, it is a critical component of a truly MR performance. While this chapter will include excerpts from the traditional score alongside discussion of specific elements, a full recreation of the score is available in Appendix B.

Page $\mid 29$ 


\section{Movement 1: Simplicity}

This first movement, Simplicity, asks the performer to consider how 'simple' interactions can

lead to very meaningful results. The performance notes for this movement read,

The first of three movements from the Touching Light suite, Simplicity is in many ways true to its name, while at the same time, like so many 'simple' things, deceptively complex. The movement asks the performer to move through a series of 'scenes' that correspond to the status of a Mixed Reality audio mixer. The mixer is rendered within a holographic volume around the performer using the Microsoft HoloLens 2 and the companion application designed for this purpose.

The following score indicates the content of each of the ten discrete channels available on the Mixed Reality mixer, and serve as a reference for the general length, shape, and timbre of each of the individual sounds. The instructions on the final page of the score designate the process by which the performer should interact with the virtual mixer, indicating the 'checkpoints' for various scenes.

In addition to manipulating the mixer, the performer is also asked to freely improvise, being inspired by the audio that is being generated by the application. The instrumentation for this movement is left to the discretion of the performer; historically, successful iterations have included those on drumset, vibraphone, and found metals. (Ian Riley, program notes from Touching Light, i. simplicity [2021])

Despite your initial reactions, this piece really is meant to be 'simple!' But, as the

performance notes indicate, 'simple' and 'easy' are not always precisely the same.

The first type of simplicity in this movement comes from the musical language that I've chosen to employ. The accompanying tracks for this movement are short, memorable, and evocative of popular dance music. Each of the ten tracks sync up on their own and repeat in easily recognizable eight-bar phrases, further simplifying the musical form. Finally, because the performer is asked to improvise along with the accompaniment tracks, I chose to center the tonality of this movement firmly in C-minor, a 'simple' key that is comfortable on a mallet keyboard instrument and fits nicely on a standard vibraphone. 

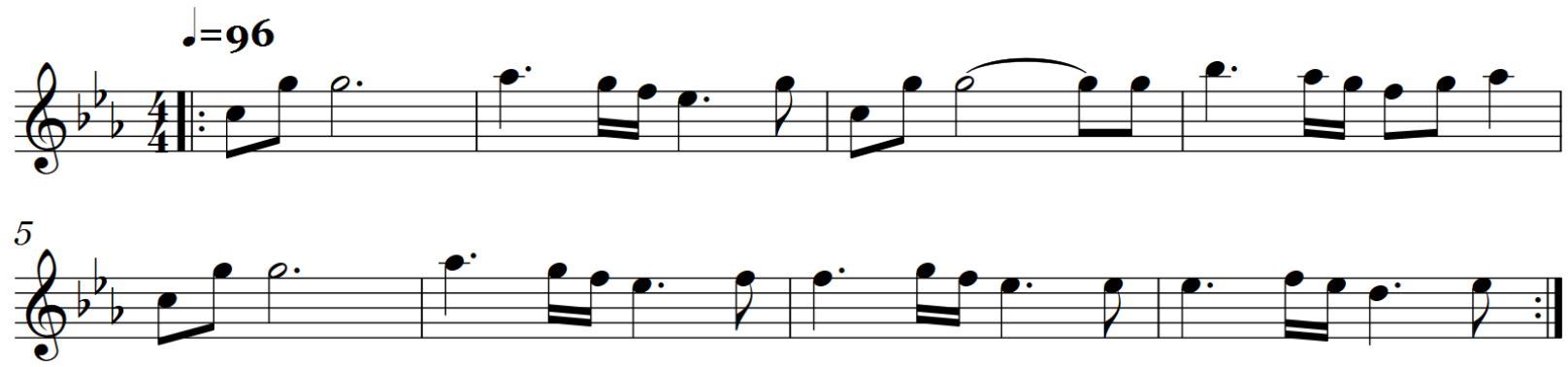

Figure 1: The 'piano' theme from Touching Light (i. simplicity)

I've included the transcription of the piano melody in Figure 1 (above). This melody is one of ten concurrent elements whose volume can be manipulated through the second sort of simplicity in this movement, the 'mixer.' The MR interactions features in this movement are meant to be straightforward and intuitive; the mixer does what you expect it to: slide a knob and the volume changes.

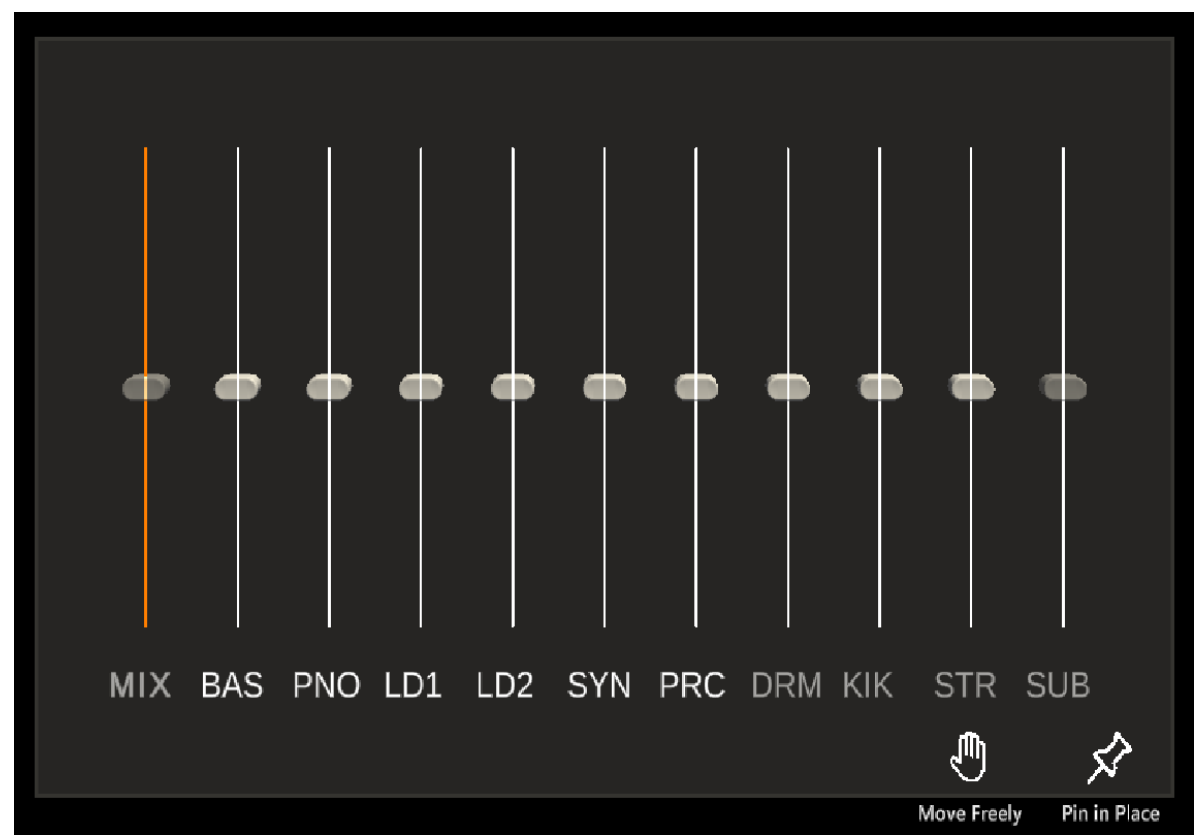

Figure 2: The Mixer from Touching Light (i. simplicity)

The interplay between the mixer and the performer makes up the crux of this movement. The performer is asked to match the settings on the mixer to a series of pictograms in the score, Page | 31 
and after matching a pictogram, listen to how the accompaniment has changed, and respond musically.

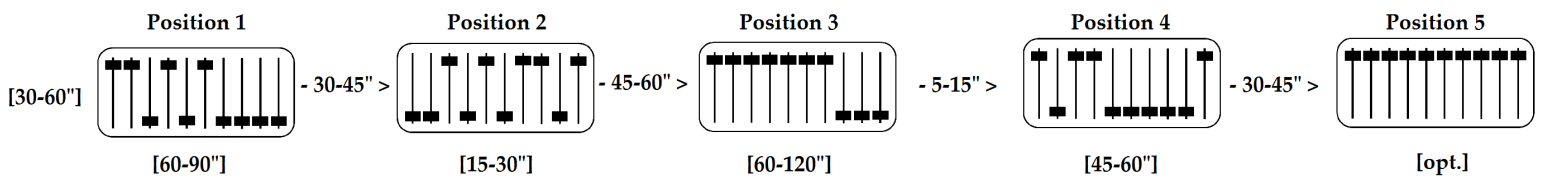

Figure 3: The five different positions (or 'scenes') from Touching Light (i. simplicity)

Each of the pictograms corresponds to a different 'state' of the mixer; the pictogram for Position 1, for instance, shows that the Bass, Piano, Lead Synth 2, and Percussion tracks should be at full, while the rest of the tracks are muted (indicated by their volume slider being all the way at the bottom).

The final element of simplicity that I considered for this movement is a simplicity of freedom. I purposefully provided explicit instructions about certain elements of the performance while at the same time, allowing the performance to rely heavily on the performer's musical intuition and ability to improvise all the way through to the end which asks the performer to decide how the movement concludes:

Position 5: Using the virtual mixer, manipulate the faders until all faders are positioned at their maximum volume. Let this combination repeat for at least 1 complete cycle, and in this time (do not decide beforehand), decide if you will take a final improvisational solo over the full track, or if you will allow the audio to be the finale. In either case, also decide how you will stop the audio (i.e., suddenly using the master fader, element by element using individual faders, etc.). Once al audio has become inaudible the movement has concluded. (Ian Riley, score instructions from Touching Light, i. simplicity [2021])

Having studied and performed nearly a dozen works for percussion that specified 'extramusical' gestures the performer must interpret (i.e., Feldman's The King of Denmark, Mark Applebaum's Aphasia, Casey Cangelosi's Plato's Cave, etc.), I noticed that there was a fine line between notation and iconography that was clarifying versus that which was convoluting. For 
this movement, I chose to present the score as a combination of written instructions paired with visual representations of the 'goals' that the performer should reach, drawing inspiration from John Cage's work, specifically his evergreen Child of Tree. To indicate the accompaniment, I combined traditional Western notation with the visualized waveforms of the audio to create a compact score (Figure 4, below). This, combined with the instructions of which Figure 3 (above) is an excerpt, compromise the entirety of the 'score' for this movement.

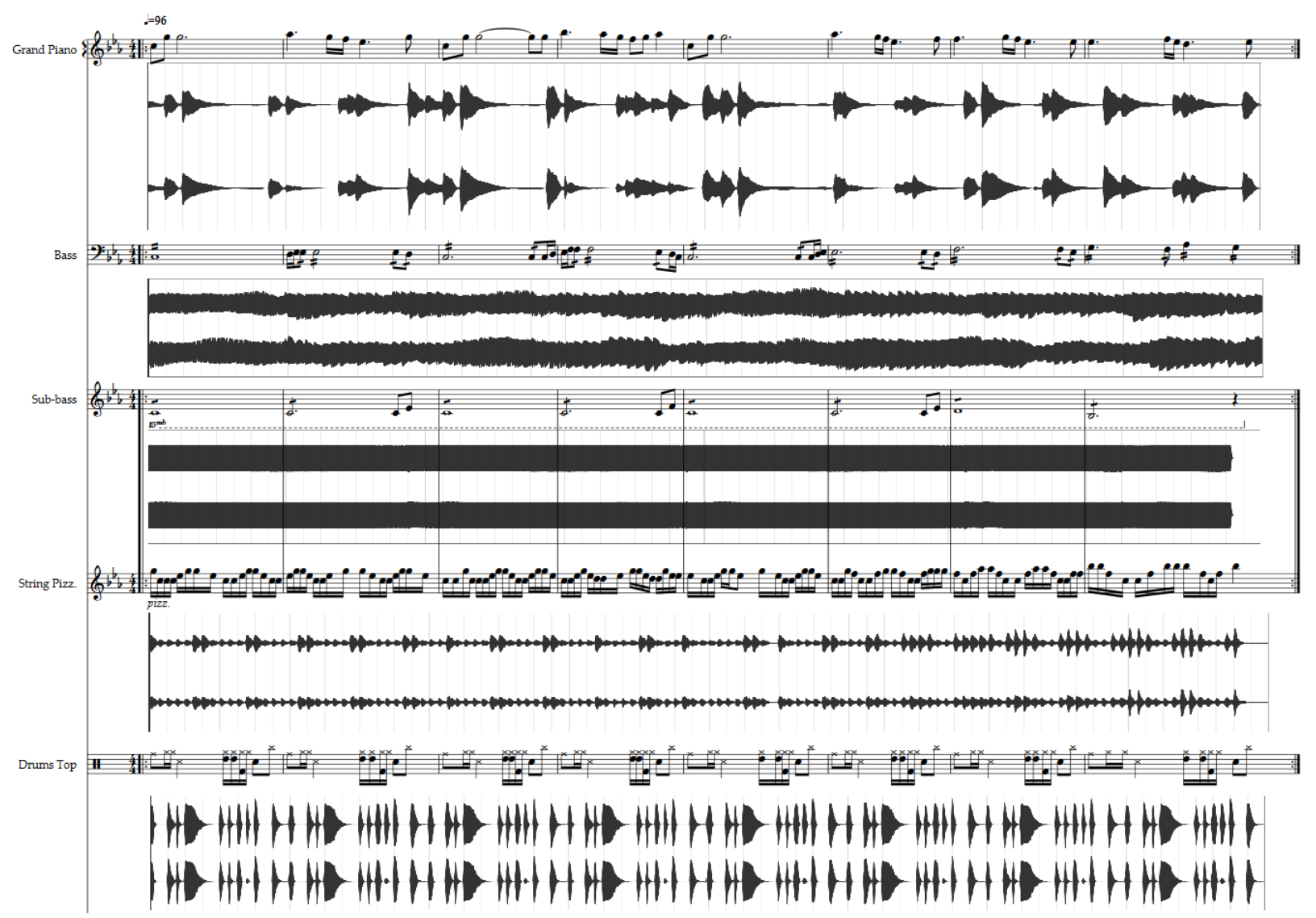

Figure 4: Excerpt from the score for Touching Light (i. simplicity)

In total, the first movement of Touching Light will last anywhere from five to twelve minutes, depending on how rapidly the performer moves through each of the prescribed scenes. Throughout, the movement depends on a collection of simple elements to communicate a meaningful musical experience: a 'simple' concept (jamming along to the 'radio'), a 'simple' Page $\mid 33$ 
score (just a set of suggestions), a 'simple' MR interface (a mixer that behaves like you expect a mixer to behave). Yet, with any luck, this combination of 'simple' elements will work together to present a complete musical experience, perhaps even reminding us in this age of complicated things, that complexity and quality are not necessarily synonymous.

\section{Page $\mid 34$}




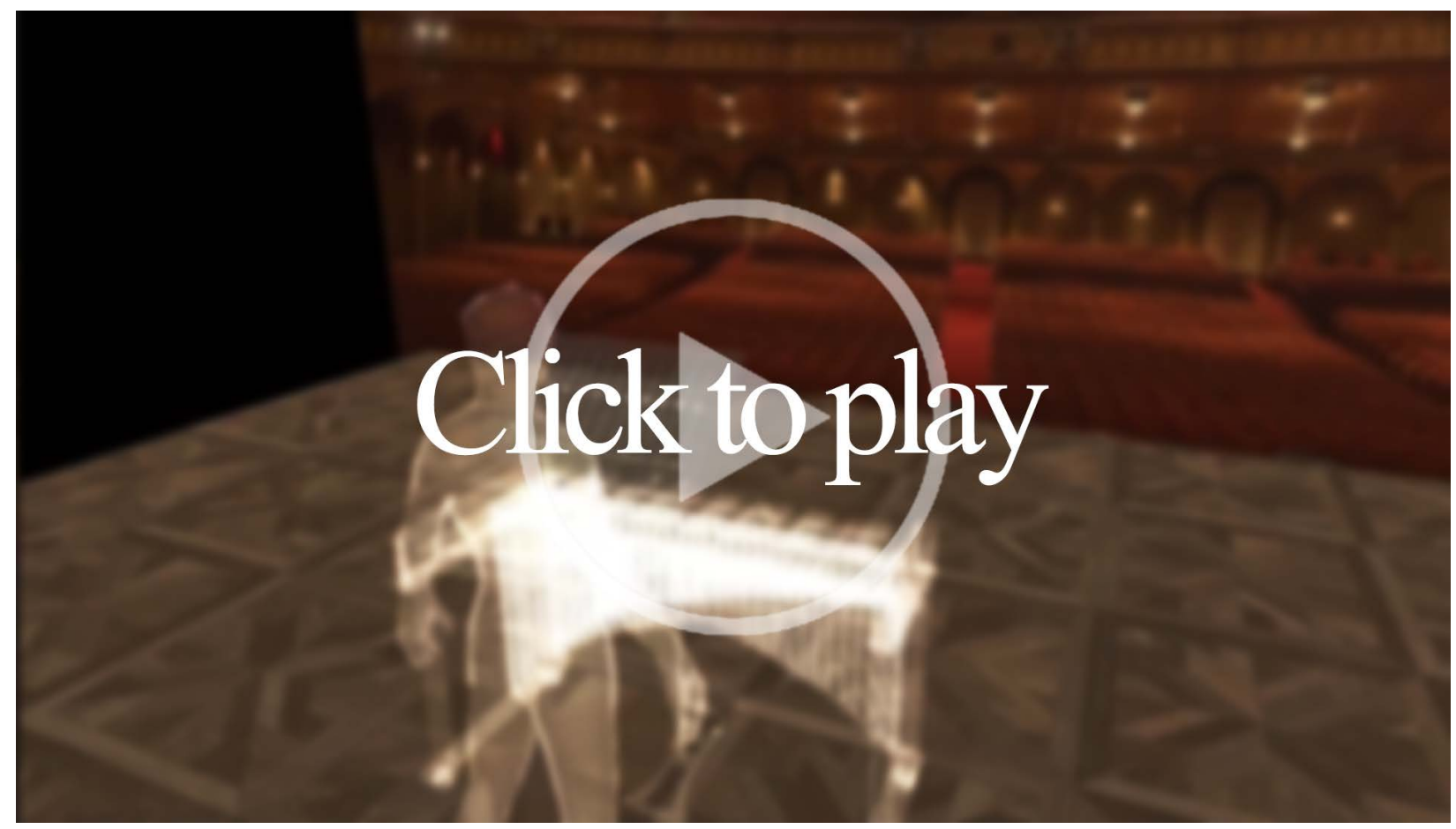

Media 1: Video demo of the first movement of Touching Light

Page $\mid 35$ 


\section{Movement 2: Soliloquy}

The second of three movements, Soliloquy is centered around a singular concept. The idea of a 'soliloquy' serves to unify the different elements, particularly the physical and virtual elements of the performance, into the same conceptual space. While Simplicity endeavored to present a collection of simple concepts with clarity and depth, Soliloquy depends much more on the performer to serve as an interpreter of the musical idea, rather than simply the score.

so·lil.o.quy (/sa 'lilakwee/) noun. an act of speaking one's thoughts aloud when by oneself regardless of any hearers.

The second movement of three movements from the Touching Light suite, Soliloquy asks the performer to freely improvise as they are inspired by a rotating carousel of landscapes from various parts of the world. In the original version, these landscapes were representational images of a lush evergreen forest, a sunset over the ocean, a snowcapped mountain peak, a far-distant nebula, and foaming surf.

The instrumentation and musical content are left up to the performer; the composition included below is simply a representative performance of Soliloquy and may be performed as written, used for inspiration, or wholly ignored in favor of original improvisational material at the performer's discretion. The intention behind the work is for the performer to respond in some way to the images within a virtual carousel around them, reciting a sort of 'soliloquy' as their own thoughts and memories about the associated memories inspire a unique musical performance. (Ian Riley, from the program notes for Touching Light, ii. soliloquy [2021])

As indicated by the performance notes above, Soliloquy focuses on the inner monologue of the performer, their imagined reality, their musical interpretation of memory and nostalgia as guided by a sort of photographic carousel as presented to them in Mixed Reality. Like Simplicity, this second movement is built upon the expectation that every performer's experience with Touching Light will be different, and each one of us will have something different to bring to this new musical medium. So, while Soliloquy does rely heavily on improvisation (as do all three movements in the suite), the composition design focuses specifically on the types of experiences that the performer will be responding to. 
The "five images" referenced in the performance notes serve as a critical element of the score but are not accessible via traditional Western notation. Instead, access these images, and therefore the score in full, requires the engagement of the MR interface. The length of this movement is left to the discretion of the performer, and parameters of the visual carousel can be altered (such as how quickly the carousel rotates, etc.) to support that decision. I've included the collection of images below (Figure 5) to quickly reference the 'stock' images that attend the original score, but a further degree of improvisation resides in the availability for the performer to replace any or all of these with images of their choice, thus further personalizing their 'soliloquy.'

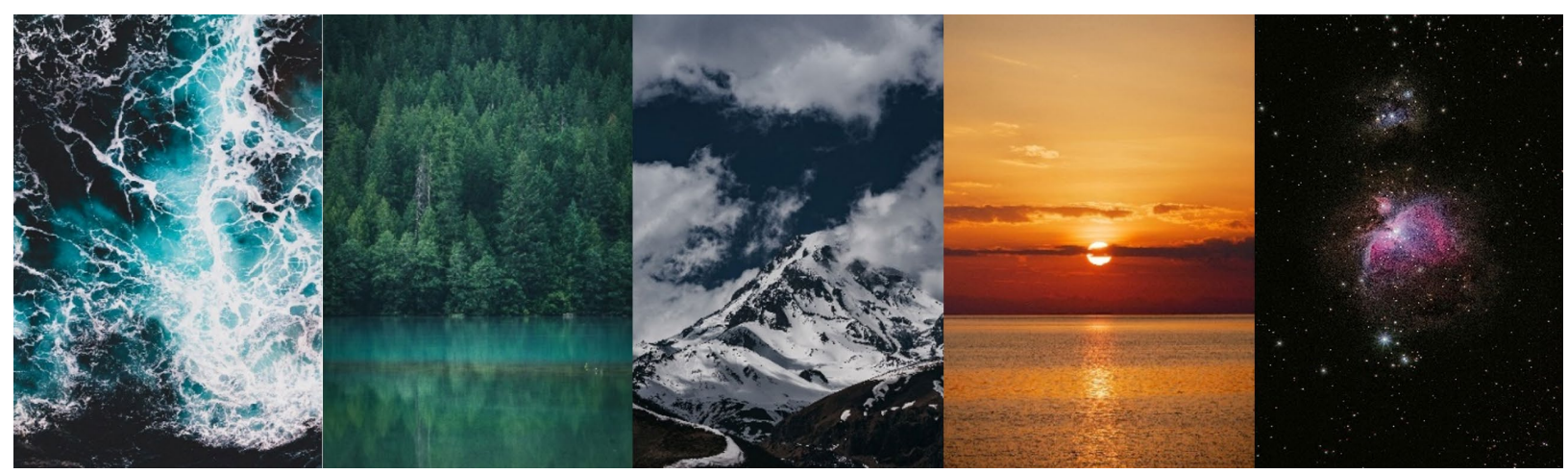

Figure 5: The five 'carousel images' from Touching Light (ii. soliloquy)

Included in the score for this movement is a transcription of my first full performance of Soliloquy. This was the first time that all the elements of instrumentation, visualization, and performance space aligned. And by focusing on these five images that are presented through the HoloLens 2 application, I was able to connect with memories of childhood and family that I hadn't considered in many years. It was a deeply emotional experience for me as an individual, and it is precisely that vulnerability, that "speaking one's thoughts aloud... regardless of any hearers" that Soliloquy seeks to convey. 


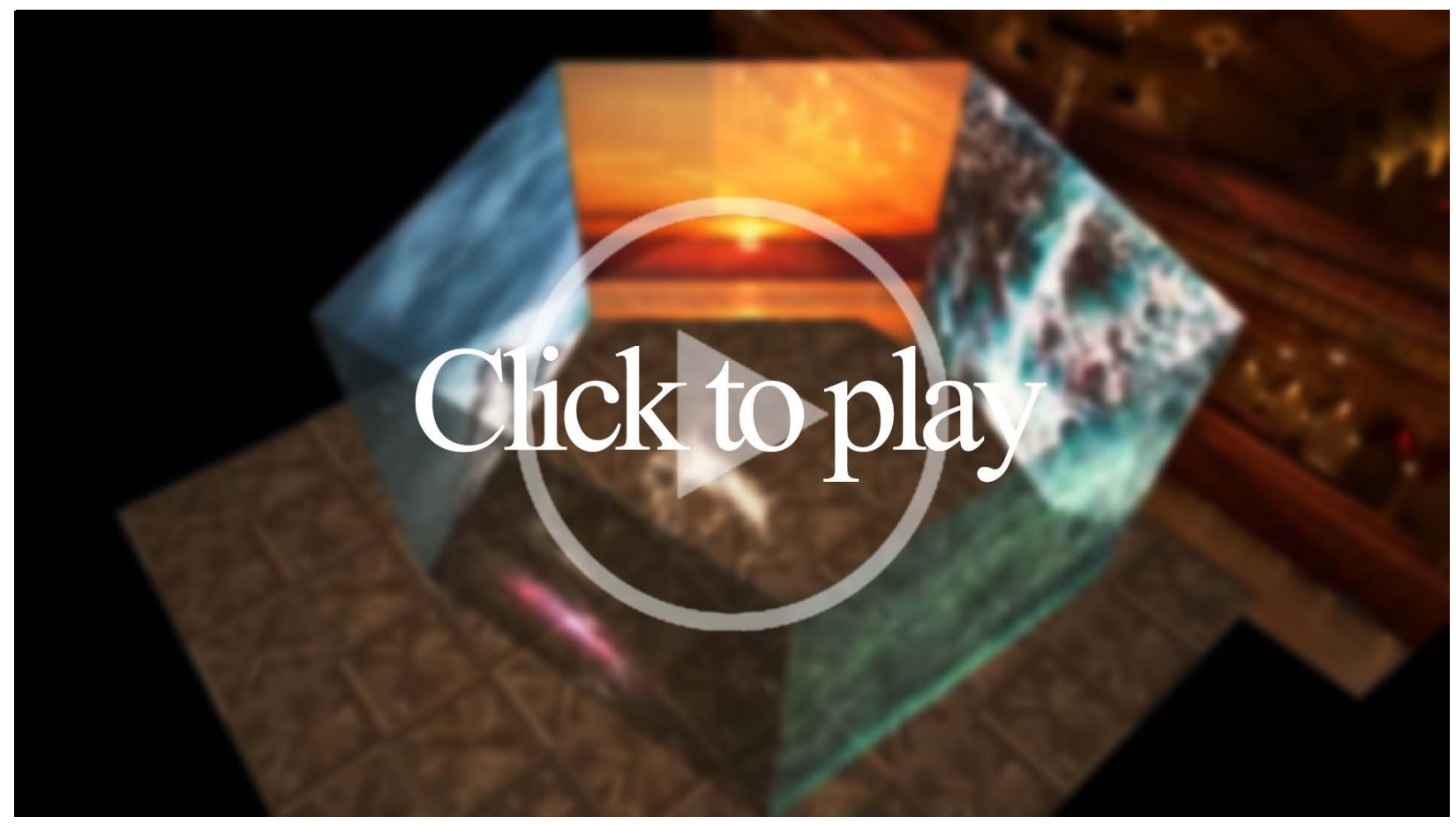

Media 2: Video demo of the second movement of Touching Light

Page $\mid 38$ 


\section{Movement 3: Synecdoche}

The third and final movement of Touching Light asks the performer to explore a holographic volume, allowing this process of discovery to generate the musical content for the final movement. Three holographic instruments are presented to the performer, and through the manipulation of these instruments in conjunction with whatever physical instrumentation (if any) they have chosen to employ, the performer will present a unique musical experience for both themselves, and the audience. The performance notes for Synecdoche read as follows,

syn'ec·do·che (/sa 'nekdəkē) noun. a figure of speech in which a part is made to represent the whole, or vice versa.

The third of three movement from the Touching Light suite, Synecdoche asks the performer to explore both representation and abstraction. Centered around musical interaction in Mixed Reality, this movement draws on essentialized elements to explore the ways that we think about music-making.

The performer is presented first with a blank canvas; unlike the first two movements, no holographic objects will enter the volume without the explicit instruction of the performer. To begin, the performer will raise their left hand, palm facing them, to access a contextual 'hand-menu.' This interaction will facilitate many elements throughout the performance of this movement.

Upon selecting a particular icon on the menu, the performer will be present with three virtual instruments within the holographic volume. Each of these three instruments have unique properties, both in comparison to physical instruments, as well as in comparison to one another.

These three instruments are visually represented by three apple-sized cubes of various colors (red, green, and blue). Each cube has a specific number of different properties as programmed by the composer, but it is up to the performer to explore and discover as many of them as possible. The more properties that are identified, the more complete the representative nature of the cubes (and thus their 'synecdoche') becomes.

As in movements one and two, the physical instrumentation that will accompany this movement is left up to the performer. With this in mind, the notational score on the following pages is presented in a combination of timbral, melodic, and graphic notation. The performer may choose to interpret any or all of these 'scores' during the performance.

While the previous two movements of this suite focused heavily on the physical instruments involved, this final movements exists firmly in shared space between the 
physical and virtual worlds, exemplifying this Mixed Reality. (Ian Riley, program notes from Touching Light, iii. synecdoche [2021])

Synecdoche, the idea that permeates this movement, focuses on the way that a small part of something may represent a much larger experience; this idea of experiencing a foretaste of what is to come inspired the exploratory nature of this movement.

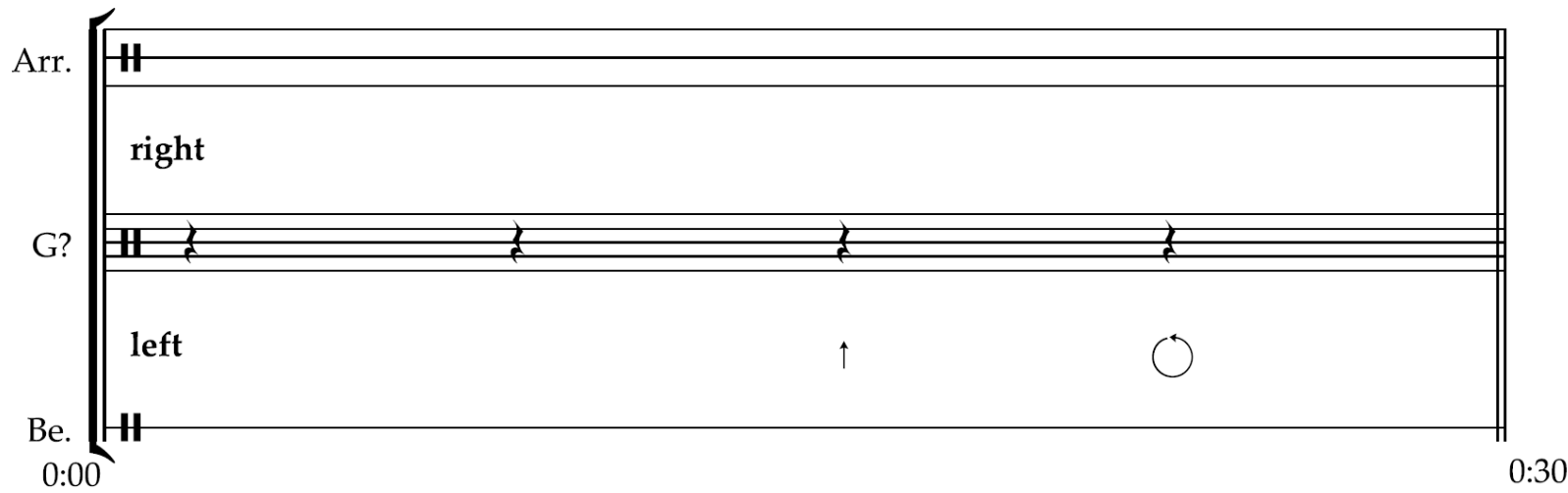

Figure 6: The first measure of Touching Light (iii. synecdoche)

Synecdoche is divided into five 'sections,' each of which feature a new type of interaction as indicated by a new notation. As indicated in the performance notes for this movement, in order to access the MR instruments (the cubes), the performer must raise their left hand ( $\uparrow$ ) and turn it counterclockwise $(\circlearrowleft)$ so that their palm is facing up. With this knowledge, the indications in the score then form a sort of synecdoche of their own, representing the larger gesture necessary to begin the work. Because the movement is meant to be primarily exploratory, no meter, time signature, or tempo is indicated. Instead, I have included suggestions on approximate durations for each set of interactions, one 'measure' always lasting approximately thirty seconds. 

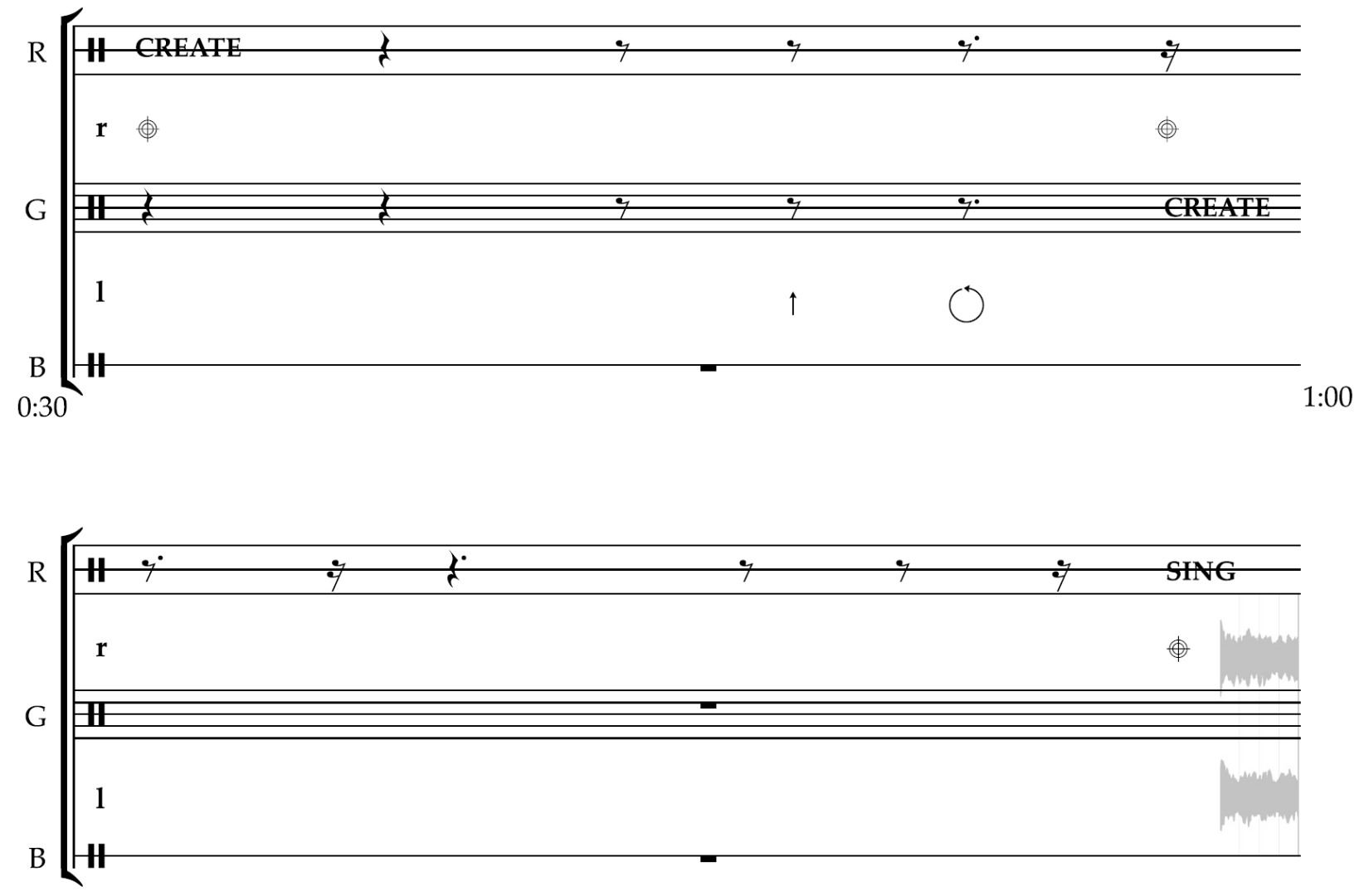

1:00

$1: 30$

Figure 7: Measures 2-3 from Touching Light (iii. synecdoche)

The second section that the performer may explore is the creation of the instruments. By selecting the 'CREATE' option on the hand menu a cube of the corresponding color will appear. These cubes are the visual identifiers of the MR instruments necessary to perform Synecdoche. Near the beginning of the first system in the Red Cube (Figure 7, above), an indication for 'selecting' the option in the menu is presented, as well as the title of the selection. Later, at the end of the second system, the option for 'SING' is suggested, which will lead the performer into the third section of the movement. 

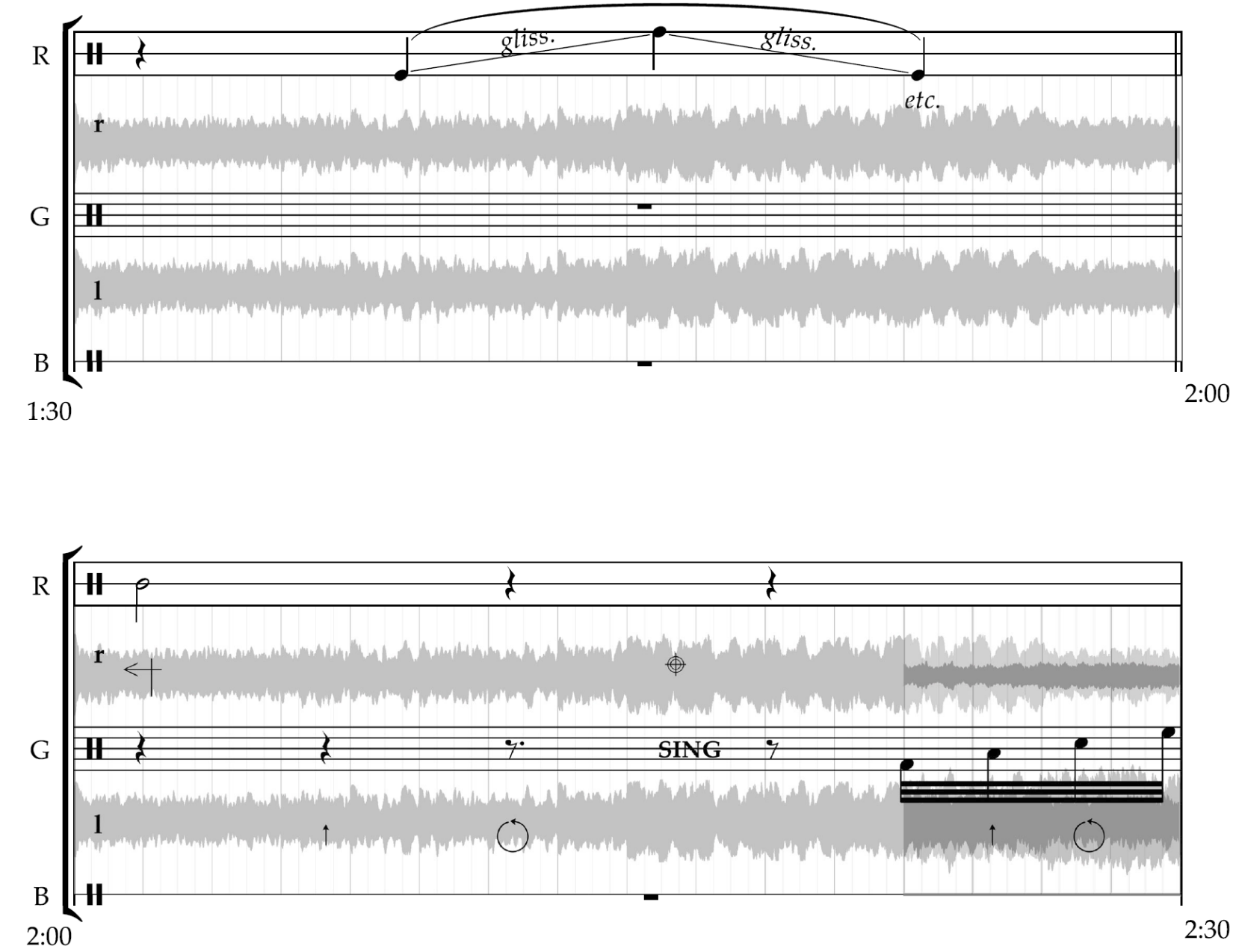

Figure 8: Measures 4-5 from Touching Light (iii. synecdoche)

Upon asking one of the instruments to 'SING,' audio will begin to emit from the instruments, as indicated by the overlying waveforms that are now present in the score. While there are options to 'SILENCE' or 'FREEZE' the instruments individually or en masse, the performance indicated by this score does not suggest those interactions through notation.

The end of the second system notates the addition of another waveform which overlaps the original RED instrument. Throughout this process, the performer may make different choices than I would, based on the same notation; this is encouraged. Conceptually, each performance of 
Synecdoche should itself serve to represent some small corner the totality of possible performances.

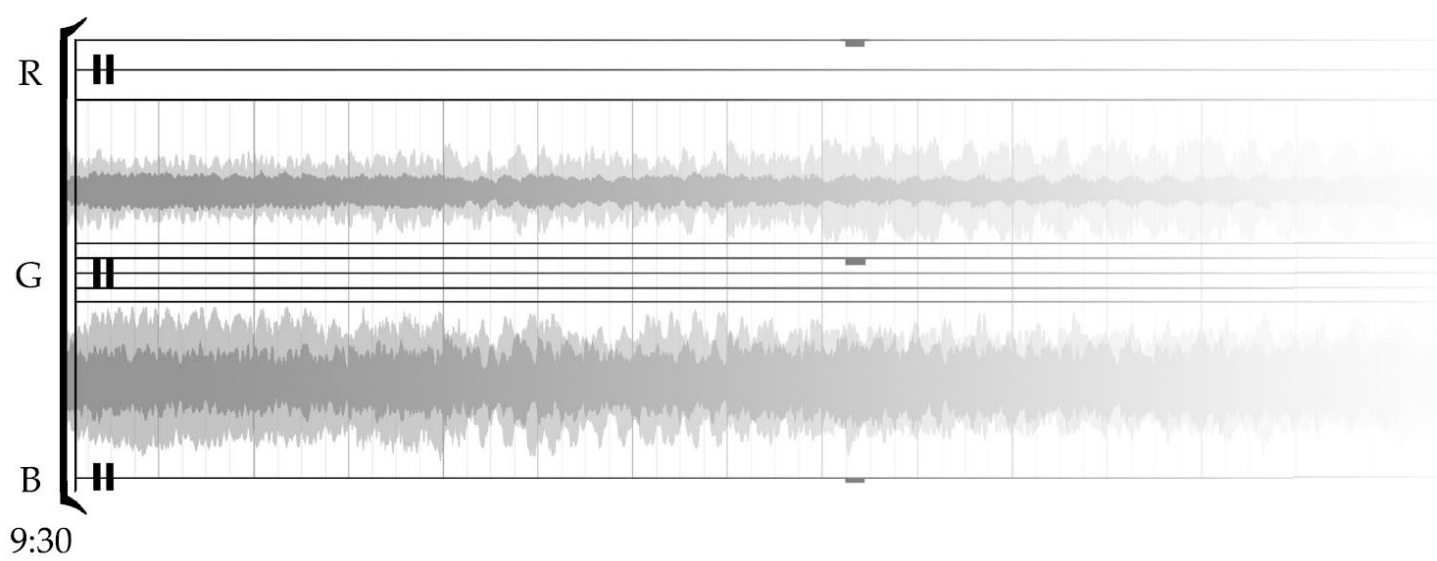

Figure 9: The final measure of Touching Light (iii. synecdoche)

The notation for Synecdoche is intentionally vague to encourage the performer to make their own decisions about how this movement should be interpreted. By combining elements of melodic, timbral, and graphic notation, I have presented a score with clear indications within a linear time frame, but the meaning of the indications themselves are left up to the performer. In this way, the performer presents a representative experience to the audience of both the process of music-making, but also the journey that I have taken in developing Touching Light. 


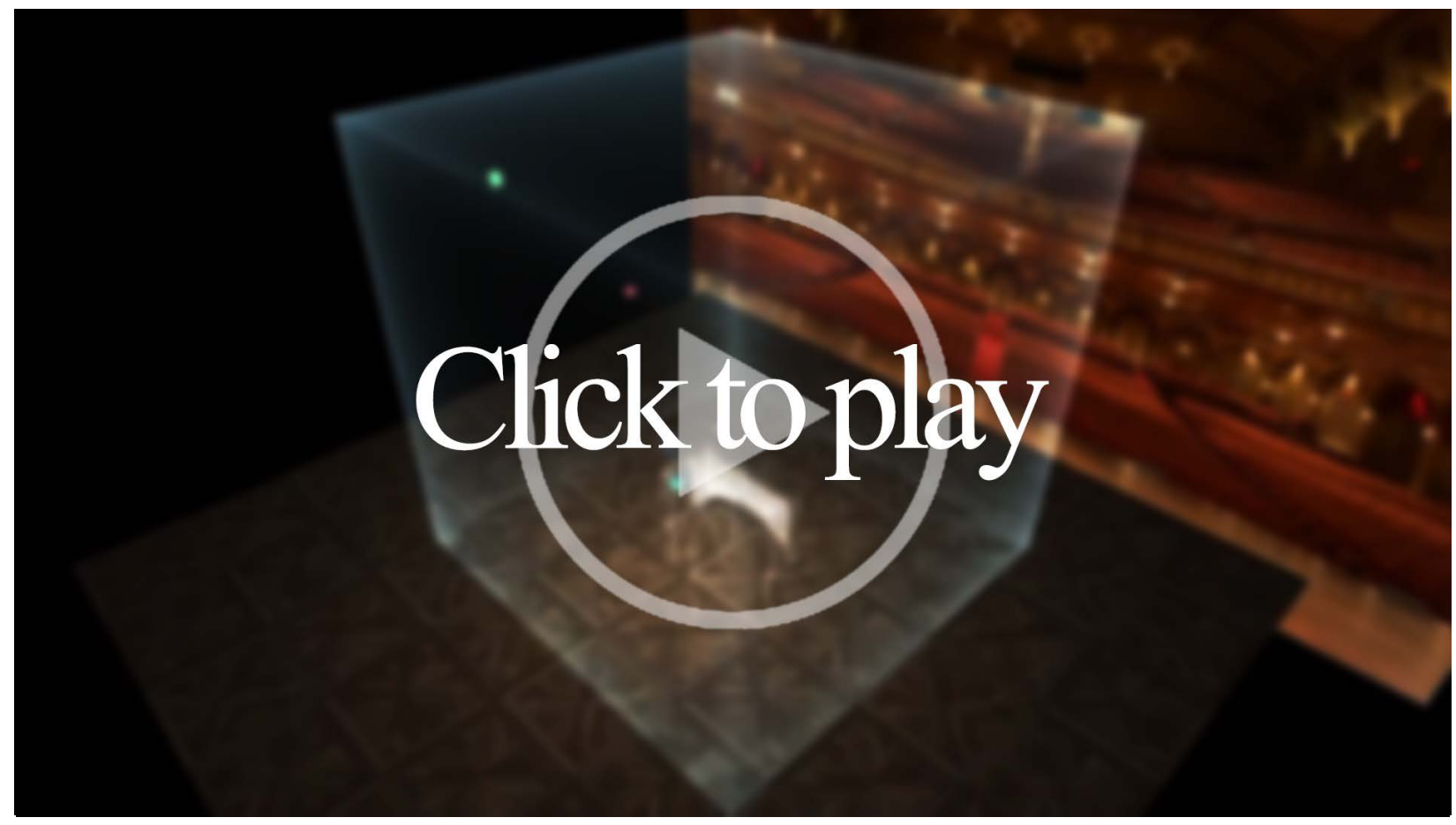

Media 3: Video demo of the third and final movement of Touching Light

Page $\mid 44$ 


\section{Touching Light as an 'Invisible Art'}

Through the process of developing, composing, rehearsing, researching, etc. for this project, several frames of reference by which to engage with MR music-making have presented themselves. As in each of the previous discussions about the individual movements in the suite, Touching Light is meant to serve as a prototypical example, a taste of what is possible when combining music-making as an artistic and communal practice and the technological developments in Mixed Reality that allow imagination to exist in a tangible way. These frames of reference, put together, form what I refer to as a 'framework music-making in Mixed Reality.'

The beginning of this framework asks the reader to consider the three foundational elements of any music-making in Mixed Reality: the user, the platform, and the compiler. As with many artistic endeavors, musical or otherwise, Touching Light began first with inspiration, and once that inspiration had taken hold, only then did I begin to consider the sorts of things that would be necessary to complete the work. What follows is my best effort to trace the progression from inspiration to development, development to composition, and composition to the performance of music, the invisible art, as made manifest through Mixed Reality.

\section{The User}

"My father is a computer programmer." $" 38$ usually lead with this as it seems to put folks at ease when they contact me, hoping that there is some secret for how I learned to work with

\footnotetext{
${ }^{38}$ With any luck, throughout this document I demonstrate an indication of my proficiencies as a 'user,' and where I might be placed upon the fluency-intuition axis we will discuss later in this chapter. At the risk of 'taking a stroll down memory lane,' I believe that these elements of my environment, experience, and education are fundamental in who I have become, not only as a musician, but for the specific consideration of this component, as a user; the same 
Mixed Reality. Yet, while his influence has certainly been a continual inspiration to me, and our shared love for technology and gadgetry has been a constant joy throughout my life, it was in fact my mother's encouragement to pursue training in the arts that positioned me to begin developing Touching Light. Despite its deep connectedness to technology, Touching Light is first a foremost a musical composition.

Early on in my academic career, I had decided to pursue a degree in English writing, with the goal of eventually teaching at the collegiate level. Knowing how much I enjoyed music while having played in the 'band' throughout middle and high school, my mother encouraged me to continue to find ways to be involved with music, whether it would be something that I pursued a career in or not. Ultimately, through connections made with music faculty at my local community college, I would eventually earn an Associate's degree in Music with a focus in music education, and a Bachelor's degree in Music Education, with foci in percussion and composition. And the rest, as they say, is history.

It was in pursuit of my master's degree that I first became deeply interested in music technology. I was fascinated by the sounds that electronic instruments could create, and that curiosity would eventually lead me to perform an all percussion and live electronics final recital during my first graduate degree. This sort of recital was a first for the small college that I was attending and, though I was unaware of this at time, something that is still uncommon in the world of contemporary percussion. Those experiences would eventually lead me to pursue a DMA in Percussion Performance at West Virginia University with a desire to continue to explore

will be true of all users, to differing degrees. The more fully a user may be identified, the more fully an MR experience might be tailored to them.

Page $\mid 46$ 
and innovate with percussion and live electronics, knowing that the faculty at WVU would both hold me accountable to a high level of musicianship while also providing unwavering support in my areas of specific interest.

\section{The Platform}

When I first started my DMA, I was aware of the work that Microsoft was doing with the HoloLens 1 (introduced in 2016), but it was approximately six months after my wife and I moved to Morgantown, West Virginia, and just four or five weeks after the beginning of my second semester in the DMA program at WVU that I saw the first marketing for the Microsoft HoloLens 2 on February $24^{\text {th }}, 2019^{39}$. I was amazed. Watching it again today still makes me smile, but I guess that's good marketing for you. As I continued my studies at WVU, I kept thinking about that video, about the HoloLens 2, and about Mixed Reality. What seemed like a pipe dream in February, making music in Mixed Reality, would become a real possibility in mind in November of that same year.

As was our tradition, the WVU Percussion Studio would attend the Percussive Arts Society International Conference (PASIC) in November of 2019, and it was during this time that through a connection with Dr. Michael Vercelli at WVU, I was able to sit down and have a conversation with Dr. Norman Weinberg, a luminary in the areas of electronic percussion and music technologies for the percussionist. It was during this meeting that Dr. Weinberg encouraged me to, 'look toward the future - to stop thinking about what is cutting edge right now

\footnotetext{
${ }^{39}$ Microsoft. Introducing Microsoft HoloLens 2, 2019. https://www.youtube.com/watch?v=eqFqtAJMtYE. 
and to start thinking about the cutting edge of the cutting edge; because that's where we're going to need people to do work.' And I knew that the future was Mixed Reality.

Beyond the obvious hurdles of learning a new technology, a few more barriers to entry still existed in the MR development space in November of 2019; namely that the HoloLens 2 was not available to purchase! Because I was not a member of the MR development community at the time, and furthermore, because I was not employed by a video game or technology company, despite my inquiries, the prioritization of a college student in West Virginia, understandably, did not take precedence in Microsoft the sales scheme.

Yet, because the HoloLens 2 was, and still is, leaps and bounds ahead of the competition in its ability to render a high-quality MR environment, I was convinced that it was this device, this platform, that would serve as the basis for what would eventually become Touching Light. When the HoloLens 2 finally became available in July of 2020 to order directly from the Microsoft Store (regardless of your development 'credentials,') I had done everything I could to ensure that I would be ready to hit the ground running when the device finally arrived.

\section{The Compiler}

Sometimes it is the mere fact that you know what you don't know that can provide the clearest path forward. Soon after the reveal of the HoloLens 2 in early 2019, the first seeds of what would eventually become Touching Light began to take root. At the time, while I had done some minimal computer programming in high school (Java, and some HTML), since I began studying music in college, I had had little time or reason to engage with the 'coding' side of technology apart from some basic formatting for websites.

\section{Page $\mid 48$}


Knowing that the HoloLens 2 would likely run on something like C\# or Visual Basic, I began thinking about other ways that I could engage with code-based music technology and would eventually teach myself how to build rudimentary circuits to trigger lighting and audio effects for a performance of Evan Chapman's Glymur in the fall of 2020, using components and resources available via the website for the technology manufacturing company AdaFruit ${ }^{40}$. Concurrent to this work, I also more fully invested myself into learning about audio recording and engineering, recording and editing my own performance videos from recitals and other concerts. Yet for all this experience, I still didn't know how to program the HoloLens 2.

When the first news of the global coronavirus pandemic entered the public awareness in the United States, it was met by a mixture of genuine concern, reasonable skepticism, and in some cases, outright dismissal. Living in West Virginia, the scope of the pandemic didn't really hit home until the University received email correspondence from university president Gordon E. Gee outlining the realities of campus closures, and the transition to online delivery for the remainder of the semester as the university endeavored to minimize the risk to the WVU community in the face of uncertain times.

There are no words to adequately describe how difficult, but likewise, how privileged an experience so many of us are in West Virginia have had; and in the face of what seemed at the time to be indefinite lockdown, I found myself able to do what anyone would do with a sudden abundance of free time... learn how to write programs for Mixed Reality!

\footnotetext{
${ }^{40}$ https://www.adafruit.com/about
} 
Over the course of the next several months, particularly during the summer of 2020, through a series of free tutorials, I learned the basics of 3-D modeling using a program called Blender ${ }^{41}$, a 3D modeling engine that is similar in many ways to the sort of interface I would eventually work with in Unity. Upon successfully ordering a HoloLens 2 from Microsoft in early July, I quickly transitioned to Unity while familiarizing myself with the sorts of gestures and interactions that drive the HoloLens 2 holographic interface.

\section{Touching Light as a 'Translucent Performance'}

With all the components finally in hand, then began the work of writing, rehearsing, and performing Touching Light. The 'Translucent Performance' methodology as described in Chapter 4 is largely derived from the sorts of challenges and opportunities presented not only to those who desire to make music in Mixed Reality but are in fact the natural outgrowth and response to experiences from long before having a HoloLens 2 in-hand.

Core to the performative practice of music, and particularly to that of the percussionist due to the unique considerations driven by the size, the disparity, and the gestural nature of our practice, the same sorts of interactions that I already employed as a performer would serve as the conceptual framework from which the three 'dimensions of translucence' would be derived. These dimensions (modeled after the three coordinate dimensions in physical space) would serve to ground my creative work in the sorts of real decisions that I already knew how to make because of my work with percussion.

\footnotetext{
${ }^{41}$ https://www.blender.org/about/
} 


\section{Length (Time and Space)}

Touching Light needed to be 'significant,' but I wanted to ensure that it was not so abstract in its application that only those performers with similar experience and similar access to equipment would be able to perform it. Thus, while Touching Light purposely exists as a significant (in the scheme of standard literature for percussion or solo instrumentation) temporal artifact, its construction as a suite in three parts allows it to be both modular and more easily accessible.

Furthermore, I knew that I wanted Touching Light to be mobile. The promise of the HoloLens 2, and Mixed Reality in general, is that there are 'no strings attached;' if you wear this device, that is all you need to enter a Mixed Reality environment. I intentionally connected that idea of mobility to the sorts of interactions and environments that the user engages throughout the work. Even Soliloquy, the second movement of Touching Light which features a large carousel of static images does not extend far beyond the anticipated 'near-field' (that which is within reach) that a percussionist will be used to engaging with. Everything in Touching Light, whether virtual or physical follows the design ethos of always being 'within reach.'

\section{Height (Gesture and Climax)}

Yet for as 'near' and as 'mobile' as the work is, it was also important to me that not only should these interactions be scalable (that is to say: adaptable to different sizes of venues and different audiences), but that the types of gestures that the user would perform should be intuitive and communicable. In designing the interaction interface for the HoloLens 2, Microsoft has already provided a collection of best practices, including ways to interact with the MR environment using touch, reach, voice, and gaze. It was upon these bones that the form of 
Touching Light rests, capitalizing primarily on physical touch gestures to ensure that the MR interactions are as accessible to a viewing audience as possible.

Similarly, when considering the musical 'height' of Touching Light, I endeavored to ensure that the level of sophistication compounded in three primary ways: visually, aurally, and conceptually. Visually, each movement engages the senses in the primary avenues of motion and color; aurally, each movement engages the senses with respect to volume and spatialization; conceptually, each movement engages the senses through the modes of complexity and emotivity.

The first movement, Simplicity features and mostly gray interface with simple lines, static motion, and repeating, contemporary-sounding musical accompaniment. The concrete idea of the first movement is easy to understand, despite being rendered in a MR environment.

The second movement, Soliloquy, overwhelms the visual sense with sight, color, scale and motion, but is tempered but a static auditory dimension. Conceptually, this movement has much greater emotional depth, and a much deeper complexity, lending it increasingly forward momentum as the movement continues.

The final movement, Synecdoche features the most rapid and most indeterminate motion as the three cubes are cast around the performance space. Paralleling this complexity, the spatialization of the audio sources further heightens the auditory dimension as the three individual tracks are submerged within the harmonically derived polyrhythms of the instrumental drones. Conceptually, this third movement's meaning is not immediately obvious, at once asking the audience to be content with the unknown, while simultaneously inviting them to explore all manner of possible meaning within the movement, as indicated by the title of Synecdoche.

\section{Page $\mid 52$}




\section{Depth (Complexity and Emotion)}

While each of the pieces of Touching Light are individually complex, and the work was undeniably complicated to put together, my sincere desire for the work is that it would tap into some part of the human experience that was intuitive, that seemed like how it ought to be; Touching Light should be magical, not only for the audience, but for the performer as well.

By co-opting the sorts of musical assumptions that we each develop as performers of Western music, I endeavored to facilitate the music-making experience in Touching Light as one that was exciting, engaging, and natural, despite how unnatural 'wearing a computer on your head to play the marimba' might seem at first.

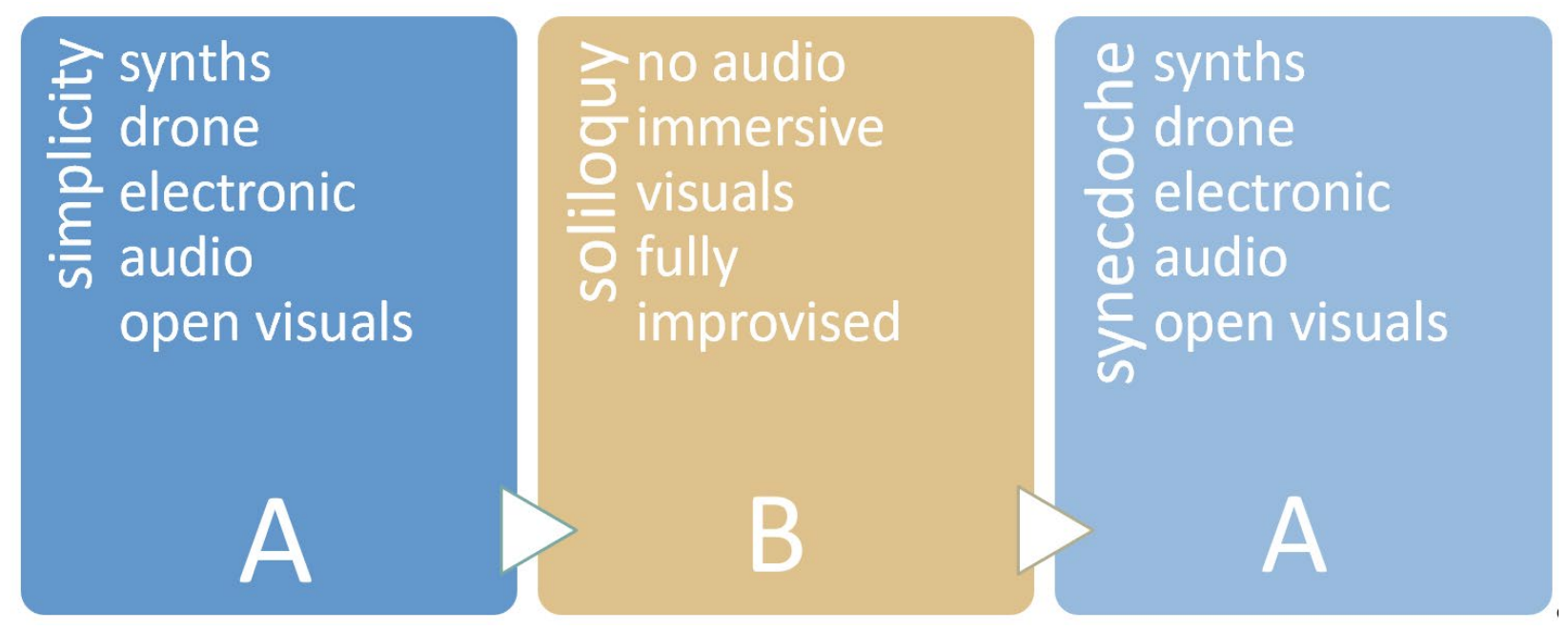

Figure 10: Diagram of Musical Form in the Touching Light Suite

I chose to arrange the motivic elements of the three movements into an easily recognizable ABA song form to further connect the musical dimension of the work to that which is familiar while inevitably asking the performer and the audience alike to revel in the unfamiliar, the unguided, and the unknown. 


\section{Rehearsing Touching Light (Engaging Liminalities)}

The rehearsal process for Touching Light has been unique in many ways. Not only is this medium of Mixed Reality performance new to me, but perhaps even more fundamentally, this medium of performance (and by extension, of practice and rehearsal) is simply just new. Where in the past I have had resources to draw on regarding best-practices, performance recordings, colleagues, teachers, and mentors who had performed the work before me, Touching Light, unsurprisingly, benefits only indirectly from those sorts of resources.

While it is true that the experience and training that I have been careful to cultivate in the areas of performance practice for percussion, and for percussion with live electronic specifically, it is instead the theoretical and methodological frameworks that I have developed in conjunction with the original work itself that have best served to provide a path toward meaningful rehearsals.

I have divided the preparation for Touching Light along the three principal liminalities discussed in more depth in Chapter 4: the tangible, the intangible, and the superposition. By orienting my practice and rehearsal time around these three vectors, I have been able to maximize my efficiency in preparing Touching Light.

\section{Tangible (Physical) Rehearsals}

The earliest work that I began focused on honing the skills and generating the creative ideas necessary to facilitate the tangible aspects of Touching Light. Because the work involves no small number of discrete musical elements and percussion techniques, taking care to work with each of them individually and with the collection of elements cohesively was critically

\section{Page $\mid 54$}


important. Touching Light has three core tangible components: instrumentation, improvisation, and interpolation.

The first step in developing the instrumentation for Touching Light necessitated considerations both logistical and artistic in nature. From a logistical standpoint, I would need to ensure that whichever instruments I chose to include, they would be immediately available to me for the performance, as well as during the period of rehearsal leading up to it. Additionally, I wanted to make sure that the instrumentation for this inaugural performance, while one of a nearly limitless number of possible combinations, provided visual, timbral, and textural interests in an acoustic sense, as well as the ability to be seamlessly integrated into the Mixed Reality environment.

For these reasons, the first movement is scored for vibraphone, the second for marimba, and the third for multiple percussion. The vibraphone will serve the necessity of melodic improvisation for the first movement, as well as blend well texturally with the EDM-inspired accompaniment. The marimba in the second movement will serve the sense of nostalgia that I hope to convey in my interpretation of the carousel of images. Finally, the multiple percussion setup for the third movement will consist largely of bells, gongs, cymbals, and other ringing metals; the unique harmonic interference of those instruments will blend deeply with the textures presented by the virtual instruments.

Because each of the three movements of Touching Light anticipate at least a limited degree of improvisation, another core process in preparing for a performance of the work was simply time with the chosen instruments, exploring melodic, harmonic, textural, and timbral extremes, and recording a series of 'scenes' onto my smartphone to review later. These improvised scenes would eventually become the musical language that I would intend to employ Page $\mid 55$ 
during a live performance. While the most authentic-seeming performance of Touching Light would be one wherein the user is freely improvising without any predetermined goals in mind, due in large part to the nature of this inaugural performance, alongside the iterative development of the score and the applications, these improvised 'scenes' function more as a creative language rather than discrete musical objects that must be explicitly recreated. In this way, they not only inform the eventual improvisation during a live performance, but also the construction and engraving of both the digital and analog scores for the work itself (see Appendix B).

Finally, a not-insignificant portion of the rehearsal cycle has been dedicated to the inevitability of some sort of eventual interpolation (unexpected interference, an instrument breaking, the application freezing, etc.) by any number of physical or virtual elements. The nature of the work itself requires that the performer's attention be perpetually split between the physical and virtual environments. To this end, I have actively interposed any number of arbitrary 'rules' to inhibit the physical rehearsals in as many, and as varied ways as possible. Whether this looks like a rule that requires me to improvise only with my left hand as I imagine that I must adjust some virtual element during the performance, or by requiring myself to switch to a different instrument or collection of instruments during an improvisation as I imagine that some unexpected interference has been generated by forces outside of my control. By rehearsing with an eye toward interpolation, I train myself to not be tied to a specific combination of instruments, instructions, etc., but to exist fully in that liminal space, that mixed reality between the physical and virtual.

\section{Intangible (Virtual) Rehearsals}

There are, of course, any number of ancillary elements that may be considered when performing in Mixed Reality, not the least of which is the necessity of rehearsing in both 
component environments to more fully prepare to engage with a Mixed Reality volume. Not unlike a physical, or 'tangible,' rehearsal, a virtual rehearsal focuses on the intangible components of the performance, things like the operating system, the gestural interface, the virtual objects themselves, and the ways that they exist in the virtual environment.

Additionally, virtual rehearsals serve to 'debug' the virtual elements as much as possible before spending the time and resources to bring them fully into the HoloLens 2, and thus into the true MR environment. Virtual rehearsals focus on two principal components: pre-build, or the time spent testing the elements within the UDE on a computer of some sort, and run-time, or the time spent testing the elements in an emulated virtual environment.

\section{Hybrid (Superposition) Rehearsals}

Ultimately, both tangible and intangible rehearsals serve the end purpose of preparing for rehearsals in Mixed Reality. If all has gone according to plan, then time need not be wasted in the MR environment troubleshooting and debugging physical and virtual elements that could have been more appropriately prepared during the early two phases of rehearsal. Because of the nature of deploying applications to the HL2, it is much less time-consuming to make small changes and tweaks at run-time where applying the change takes on a few seconds than after deployment to the HoloLens 2 where each change requires upwards of 30-minutes of transfer

time between the compiler and platform for any changes to be made. Due to this time lag, hybrid rehearsals serve a dual purpose.

Once the application is deployed to the HL2, chances are, for the first few iterations, there has been something missed during the virtual rehearsals. Maybe more than one something. For this reason, immersion time (that is, the time that the user spends in the MR environment) is critical, as the more time you spend immersed, the sooner you will notice things that are not Page $\mid 57$ 
working properly. Part of this rehearsal process, not unlike rehearsing with an ensemble, requires that, at times, you move on, making a note of what needs to be fixed without letting it completely derail the rehearsal. This final troubleshooting step during rehearsal is more about generating a list of all the possible issues (as well as imagining what issues might also exist as precursors to what is currently going wrong), and then addressing them en masse before deploying the next version of the application.

Yet, for as thrilling as immersion troubleshooting might sound, it is not the primary goal of hybrid rehearsals; rather, this is reserved for an experience that I have come to think of 'making magic.' It might sound corny, but there is something deeply satisfying about seeing all the carefully curated elements of any performance come together; yet when each of those individual elements were meticulously crafted by your own hands, it is truly a magical experience when everything works the way that it ought to. But it is also at this juncture that it is most necessary to not lose yourself in the immersive space, but rather to focus on the combination of elements, and adjudicate, as objectively as possible where the 'magic' is working, and where it most definitely is not. 


\section{Performing Touching Light}

In final preparation for the performance, there were still a few obstacles to overcome, almost all of which having to do with making Touching Light as accessible to the audience as possible. Unlike an exclusively physical or exclusively virtual performance, Mixed Reality provides a unique opportunity for the user to engage with both spaces simultaneously, but the limitations of the current technology required some creative problem-solving in order to perform music in Mixed Reality in a way that a non-expert audience could immediately understand. The first layer to mitigating this barrier was projection. Somehow, the audience needed to see what I'm seeing, to hear what I'm hearing, and the most cost-effective way to do that was through some sort of live feed from the HL2 to an on-stage screen or display.

\section{Projection}

Barring the ability to have each individual member of the audience wearing their own HoloLens headset and viewing the scene along with me, I devised a way to project my view (the 'first-person' view) of the environment to a screen on-stage with me. This allows the audience to have a sense of the virtual environment with which I am interacting along with an uninterrupted view of the physical environment as per normal. 


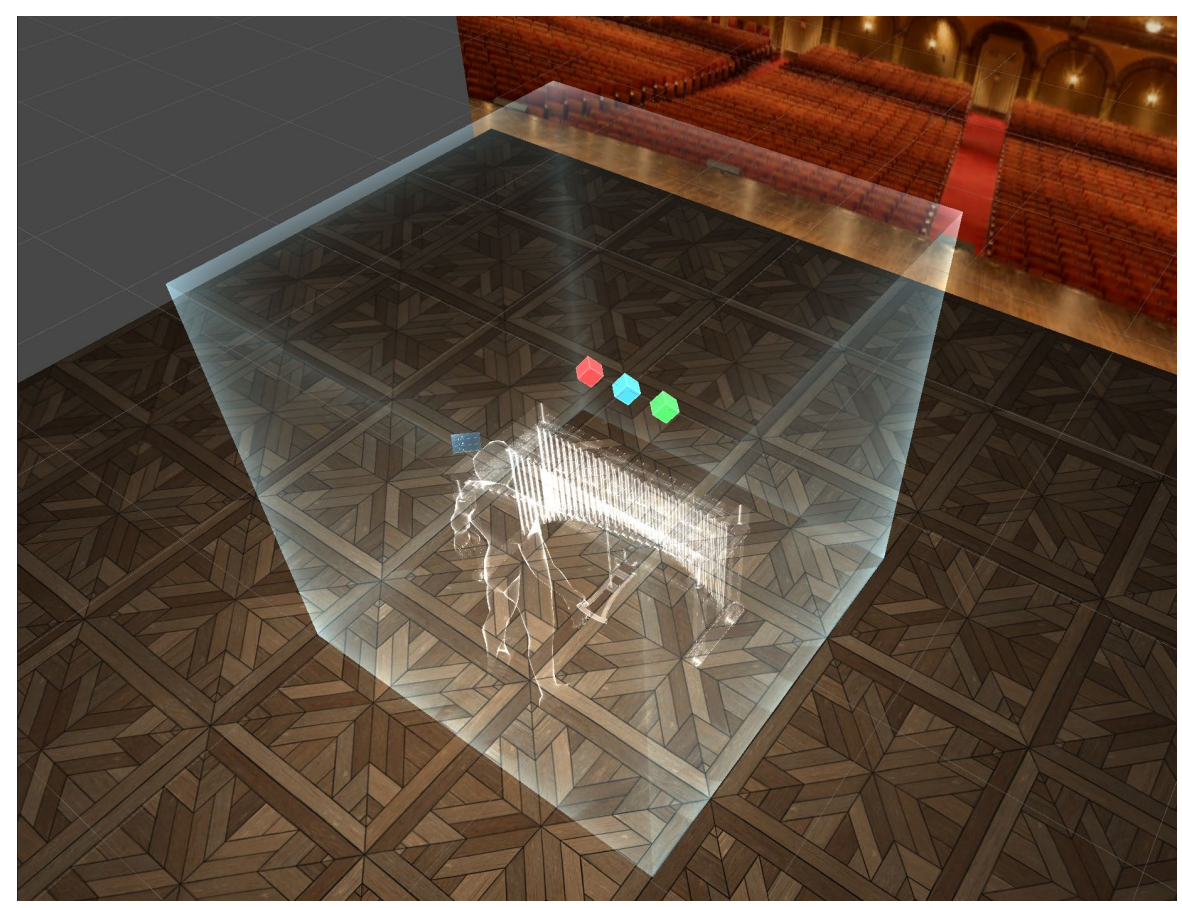

Figure 11: Representation of the MR environment in Touching Light (iii. synecdoche)

Figure 11 (above) shows, from a birds-eye view, the components of the MR environment as a sort of diorama. The figure and the vibraphone represent physical elements (along with the floor and the 'audience'), while the three colored cubes and the large 'cage' are completely virtual elements. 


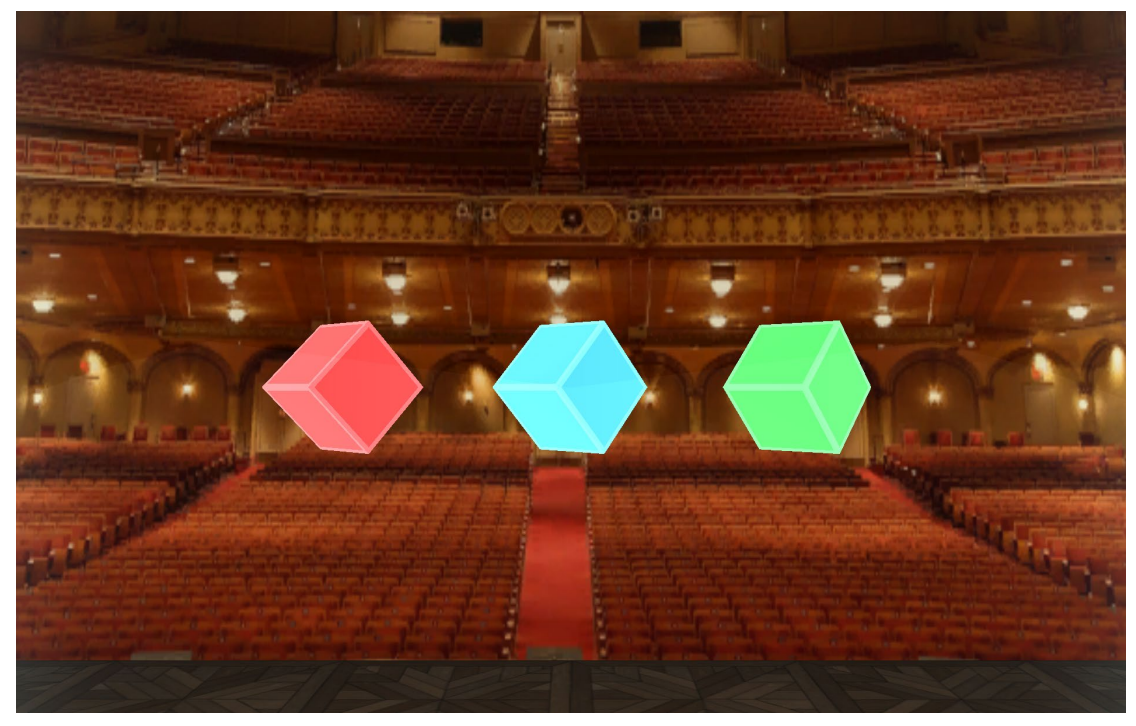

Figure 12: Representation of the User's View from Touching Light (iii. synecdoche)

Figure 12 (above) presents an approximation of what the performer's view will be from stage: three colored cubes hang in the air in front of them, floating above a physical instrument to collection of instruments.

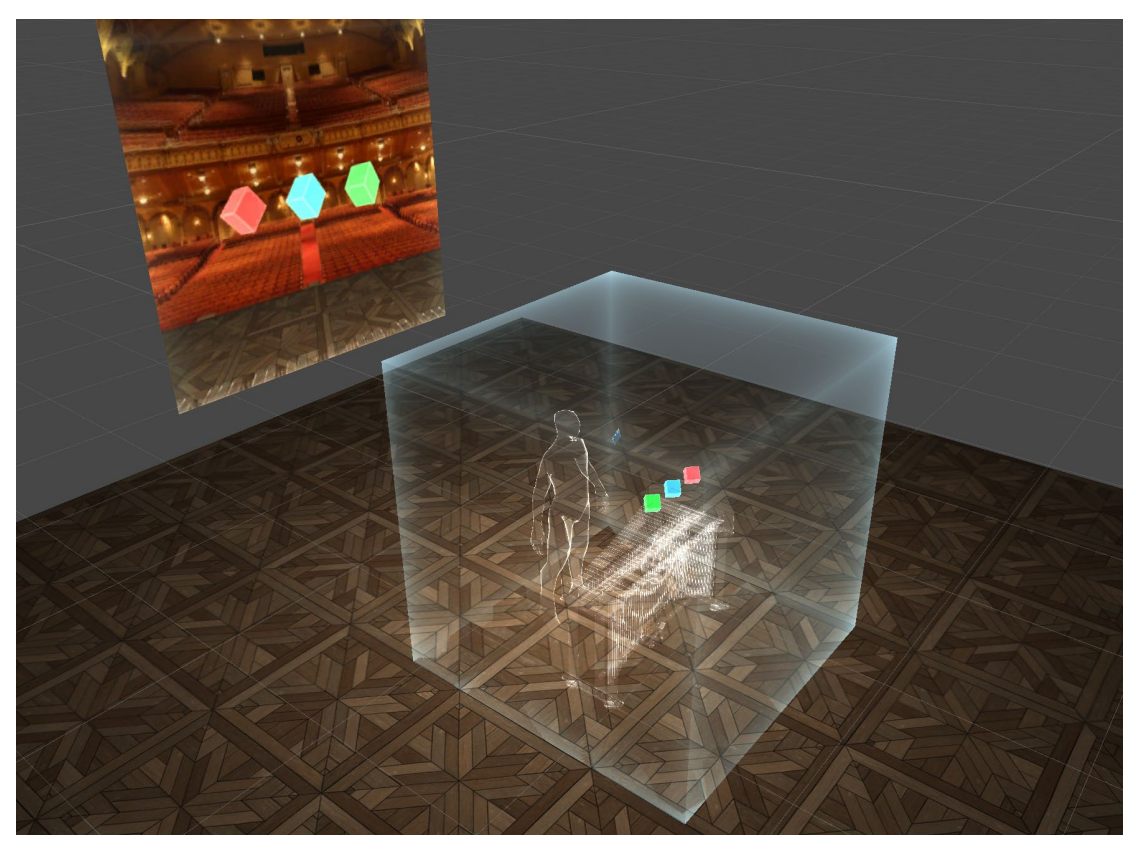

Figure 13: Representation of the live feed from Touching Light (iii. synecdoche) 
Figure 13 (above) shows a birds-eye view of the complete MR environment, as well as a representation of the screen at the back of the stage which will be seen by the audience. On this screen is the first-person viewpoint of the performer.

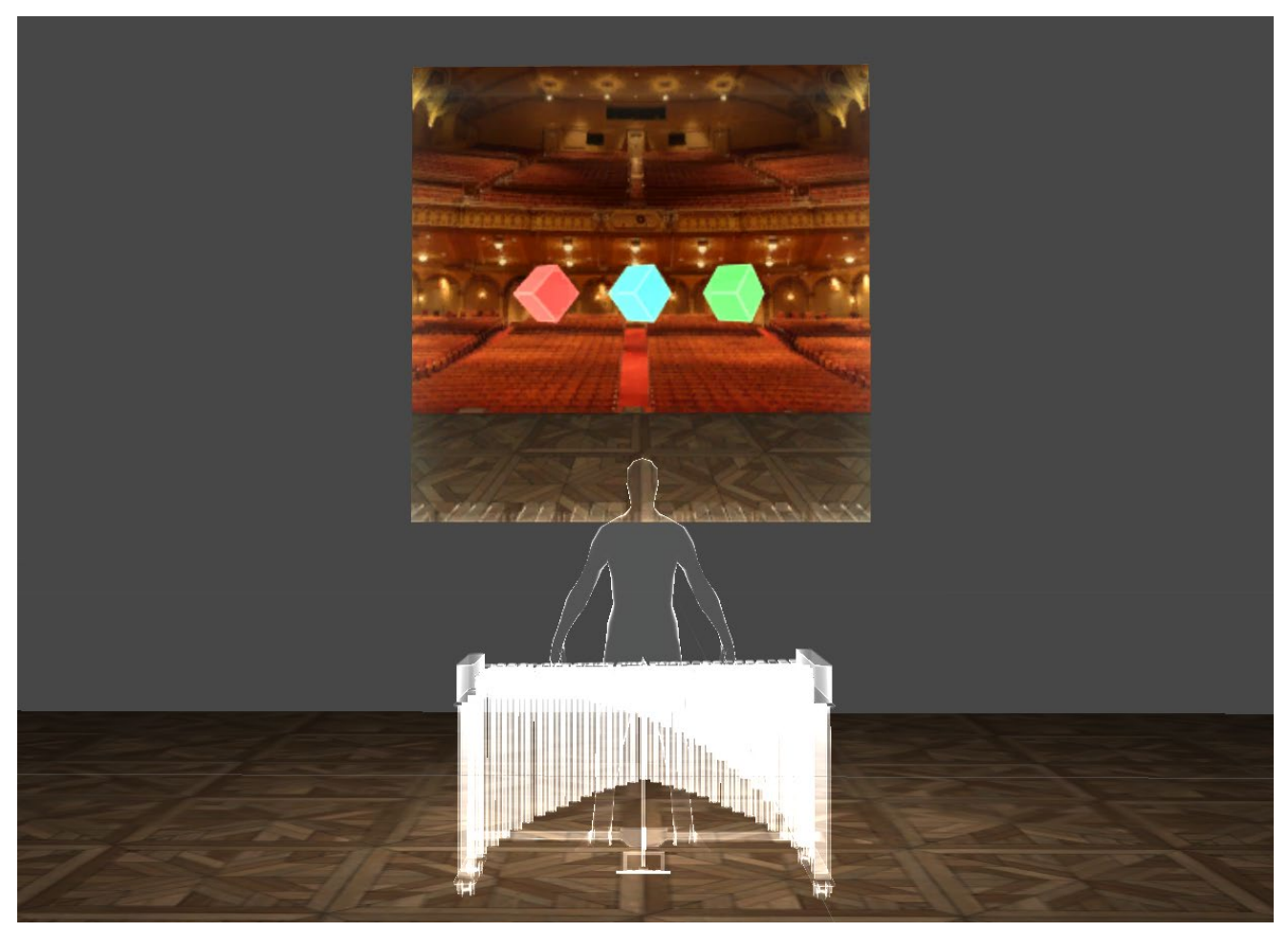

Figure 14: Representation of the Audience's View from Touching Light (iii. synecdoche)

Figure 14 (above) presents a diorama of what the audience will see from their perspective: the performer and any physical instruments on stage, backlit by a screen that will show the performer's first-person view, inclusive of any virtual objects within the MR space with which the performer will interact.

\section{Program Notes}

The second principal way to communicate accessibility to the audience is through the provision of carefully crafted program notes. While the typical collection of program notes may sometimes be lacking, due to the newness of the music-making in Mixed Reality, the program 
notes for Touching Light (Appendix A) make every effort to explain the various components of the work in clear, intelligible, colloquial language, further increasing the accessibility to the performance through their construction.

\section{Pandemic Accommodations}

Touching Light has been carefully designed to maximize the performer's ability to connect with the audience, an affordance that is unique in the world of virtual computing due to the nature of Mixed Reality as a medium. The marriage of the physical and virtual worlds is so inherent not only to technology, but to the intention behind both this document as well as this original work, that it is with particular care that I must navigate the current realities considering the global pandemic and the necessary restrictions that this reality poses for live music performance.

At WVU, we are incredibly fortunate to have access to performance and rehearsal spaces that can accommodate large ensembles safely, something that many musicians throughout the world do not have access to. Yet even with these accommodations in place, it has still been necessary to significantly limit the attendance of live recital performances held at WVU; it is easy to imagine how this could severely handicap a performance of this nature.

Yet, it is in fact with great excitement that I look forward to presenting Touching Light to a small live audience, and a much larger virtual audience via live streaming and archival video recordings. Perhaps now more than any other time in history, the world has learned to depend on technology to bring us together, and it is precisely this conjunction of the physical and the virtual, of this shared liminality, of this mixed reality, that so continues to captivate my imagination. 
It is my hope that, rather than 'in spite' of current restrictions, but instead, 'in light of' those same accommodations, Touching Light will serve as an opportunity to see the world through new eyes, to hear the world through new ears; ones that see beyond simply what is around us, and into the shared imagination that we have always dreamed of. That somehow, regardless of distance, or hardship, regardless of limitation or circumstance, that we might discover some critical part of ourselves anew by 'touching light.' 


\section{Chapter 4 - Visualizing the Invisible Art}

Considerations for Developing a Digital Score for MR Music-Making

Throughout the development process of Touching Light, my goal has always been to discern what musical and logistical elements present themselves that are unique to music-making in Mixed Reality. My hope is that these considerations will help to encourages others to explore this medium. To that end, in this chapter I will present a collection of three distinct 'frames' through which the development of a Mixed Reality musical performance might be better understood. Together, these three frames form a 'framework' through which any MR musicmaking, whether an original MR composition or an existing work adapted for MR, might be contextualized. The ultimate goal of this framework is to suggest methods of deepening the musical elements of MR performances through careful consideration of those elements which are unique to Mixed Reality.

\section{Three Components of a MR Score}

Music is, by its most fundamental nature, an invisible art. Based principally in the construction of intentional sound, music is often the focus of discussions about locality and definition. This ambiguity is further complicated by the many varieties of a musical 'score,' and its definition. Is the 'score' the music? Does the score reference some musical object? In broad terms, perhaps we may agree that a score is a set of instructions that helps to facilitate the production of a musical performance. By agreeing on these characteristics, we may begin to see how a score for Mixed Reality, which in and of itself is a blending of physical and virtual elements, will likewise require a blending of those same elements to be most effective. 
To address the unique considerations of the MR medium, a Mixed Reality score is made up of three critical components: the user (or the 'performer'), the platform (or 'the device'), and the compiler (or 'the code-writing program'). These three elements will be unique in every performance, if for no other reason than that the user in each performance will never be precisely the same. Furthermore, considerations of compiler and platform, while closely related, should be resolved individually, as each serve a specific role in the creation of the MR Score.

These three components are 'critical' in the sense that failing to consider any one of these three elements is a non-starter for music-making in Mixed Reality. These are the principal components of the MR score, the vehicle that is interpreted by the performer to generate the music.

\section{The User (fluency v. intuition)}

Perhaps the most critical component of any MR score is the user; without their natural blending of (virtual) imagination and (physical) interaction, the score cannot be realized. While any number of considerations might be made about the user, the one that I find most fundamental is an understanding of how the score may be designed with the user's fluency in mind.

If we conceive of the user somewhere along an axis which at one end has a user with utmost fluency in MR music-making (and thus can engage with a score that requires a high degree of fluency) and at the other end, a user who fluency is near-zero (and thus can engage with a score only so long as all of the elements are perfectly intuitive), we begin to see how considering this range of users may help to inform the presentation of the MR score.

Touching Light is designed primarily as a work that I will perform, and so makes certain assumptions about its accessibility regarding the fluency of its 'anticipated user.' While I expect 
that I will have the most innate level of fluency with the score for Touching Light, considerations of accessibility and intuitive design are fundamental to the development of the user-experience for any MR experience.

Given the limited scope of current music-making in Mixed Reality, there exists an opportunity to develop both compositional and pedagogical practices that standardize a 'universal fluency' approach for MR interactions. At the current juncture, and certainly for Touching Light specifically, a fundamental level of technological proficiency and platformspecific gestural literacy is required to effectively engage with the medium. Yet by considering this fluency-intuition axis there is a great potential for the development of the medium of musicmaking in mixed-reality to evolve in a way that is inherently accessible.

In the case of Touching Light, while I was the anticipated 'user' in this three-part model of user-platform-compiler, I endeavored to ensure a balance along between fluency and intuition. It would be easy to create a musical work where all the gestures and interactions would seem to be deeply intuitive (to me), while in fact the level of fluency required would just so happen to be exactly the level of fluency that I would already exhibit. When considering the 'anticipated user' of the digital score, interrogate the level of accessibility presented by the current state of the score:

- Is it intuitive or does it require a certain degree of fluency?

- What sorts of accommodations can be made to the score to make it more accessible?

- How much freedom is granted to the user to 'perform' in Mixed Reality?

- If a certain fluency is required, how is that communicated to the user?

- $\quad$ Can it be musical? 


\section{The Platform (universality v. specificity)}

This second component in the consideration of scoring for Mixed Reality is the one which will be most readily associated with the technology, the platform. The 'platform' is simply the device which the performer will use to 'enter' the MR environment and will be the primary means by which they will interact with MR elements once in that space. While Touching Light capitalizes on the advances in MR technology present in the Microsoft HoloLens 2, the consideration of the balance between universality and specificity is applicable to any MR platform.

The endpoints of this axis consider how easily transferable a given score is between different platforms (e.g., the HL2 v. the Oculus Quest 2, etc.). Creating a score that is maximally universal, as is the case for any score with 'flexible instrumentation' (e.g., Terry Riley's In $C^{42}$ ), specificity is sacrificed, and with it, any of the unique interactions that those specific limitations might enable. Inversely, by creating a score that is maximally specific, the application of the score to other instrumentations requires additional effort on the part of the performer (e.g., transcribing the Bach Cello Suites ${ }^{43}$ for marimba).

Despite the intentional limitations of Touching Light, similar considerations are necessary for any work involving Mixed Reality. While the HoloLens 2 is, to date, the most functional MR platform for this sort of engagement, it is not the only option, and will undoubtedly be joined by

\footnotetext{
${ }^{42}$ Riley, Terry. In C. Celestial Harmonies, 1989.

${ }^{43}$ Stevens, Leigh Howard. Six Suites for Marimba. Keyboard Percussion Publications, 2012. 
any number of devices in a similar category as early this year ${ }^{44}$. A great boon of MR technology is the universality and interchangeability of much of current MR and VR technology. While Touching Light is designed directly and exclusively for the HoloLens 2, with minimal alterations, a comparable digital score could be adapted for any number of other VR, AR, or MR platforms.

In considering the axis of universality-specificity, certain questions may clarify which platforms would best serve the performance experience:

- Is it necessary for the platform to be aware of the physical environment? (if so, a device with outward-facing sensors will be necessary)

- Is it necessary or beneficial for the user to be aware of the physical environment? (if so, then an opaque device, like most fully enclosed VR headsets, is not a good fit)

- Is it important for the user and platform to be mobile during the performance?

(if so, then any device that requires a cable tether is inadvisable)

- How much processing power will it take to render the MR environment? (if it is significant, then an untethered solution may not be feasible in 2021), etc.?

\section{The Compiler (dexterity v. compatibility)}

This final component, the compiler, is the one that is least commonly understood, and therefore may require the most careful consideration for those musicians who are new to working with Mixed Reality. Essentially, the compiler is the program used to design, build ('compile'), and transfer the virtual elements of the MR score to the platform so that they may be engaged by

\footnotetext{
44 “It’s Better Virtual: AR/VR Takes the Stage - CES 2021.” Accessed January 29, 2021. https://www.ces.tech/Articles/2021/January/It\%E2\%80\%99s-Better-Virtual-AR-VR-Takes-the-Stage.aspx.
} 
the user. While many of the considerations about compilers come down to the specific background of the user (if they can code in one language or another) or the requirements of the platform (if it requires a specific compiler), we may also consider compatibility more generally.

If we imagine that a maximally compatible compiler is one that is platform and user agnostic, just as in our considerations of those other components, there will always be a tradeoff. In this case, the inverse of compatibility might be called 'dexterity,' or the ability of the compiler to perform a multitude of specific tasks quickly and efficiently because it is built specifically for the scenario at hand. If a compiler is not sufficiently dexterous, it may not be able to execute certain functions at all, but if it is not sufficiently compatible, this may limit the performance in different ways altogether.

Another way to think about dexterity is through the lens of financial cost. Many modern compilers (Unity, Unreal, etc.) have free-to-use licenses which make them very 'dexterous' in contrast to expensive programs like Maya ${ }^{45}$ or AutoCAD ${ }^{46}$. While the latter may have greater compatibility, often their financial cost is not worth the trade-off.

One final lens through which to view this comparison is through that of user and platform. Because the compiler is the program that will literally 'compile' the MR environment, it serves as a sort of intermediary between the user and platform. By better understanding the user's fluency and the platform's specificity, a more informed choice can be made about the compiler. Touching Light is developed in Unity not only because it is free to use, but primarily

\footnotetext{
45 “Maya Software | Computer Animation \& Modeling Software | Autodesk." Accessed January 29, 2021.

https://www.autodesk.com/products/maya/overview.

${ }^{46}$ https://www.autodesk.com/products/autocad/overview

Page $\mid 70$
} 
because I as the user was familiar with the interface from working with similar programs in the past, and because the MRTK (the Microsoft Mixed Reality Toolkit), and by extension the HoloLens 2, integrates directly with Unity.

When considering which compiler to use, ask questions like:

- How does the user's fluency affect this decision?

- Does the platform have specific requirements?

- How much time, energy, and finance am I interested in investing?

- Do I need help?

This last question is one that is perhaps not asked soon enough, particularly when engaging with new musical technologies. In most cases, musical training does not include courses in computer programming or coding, and only recently have music technologies courses been offered, let alone required, as part of an undergraduate curriculum in music. But one of the most exciting elements of MR music-making for me has been the collaborative experiences that I've had with other technologists and musicians during this process. MR as a music-making medium has great potential to connect two traditionally disparate communities (music and technology) that may not naturally collaborate in this way outside of this creative space.

\section{Visualizing These Component Axes}

The various considerations of each of these components will help to guide the MR musicmaking experience. Regardless of the unique combination of components, these critical axes can be manipulated to best accommodate to provide the most effective musical interactions. At the current moment, the Microsoft HoloLens 2 is the de facto platform for truly cutting-edge MR interactions, but this may not always be so. 
And while it is my opinion that the unique alchemy provided by the Microsoft HoloLens 2, the Unity Development Engine, and the fluent composer-performer provides the greatest level of potential for music-making in Mixed Reality, this framework is applicable to any number of other combinations of user, platform, and compiler.

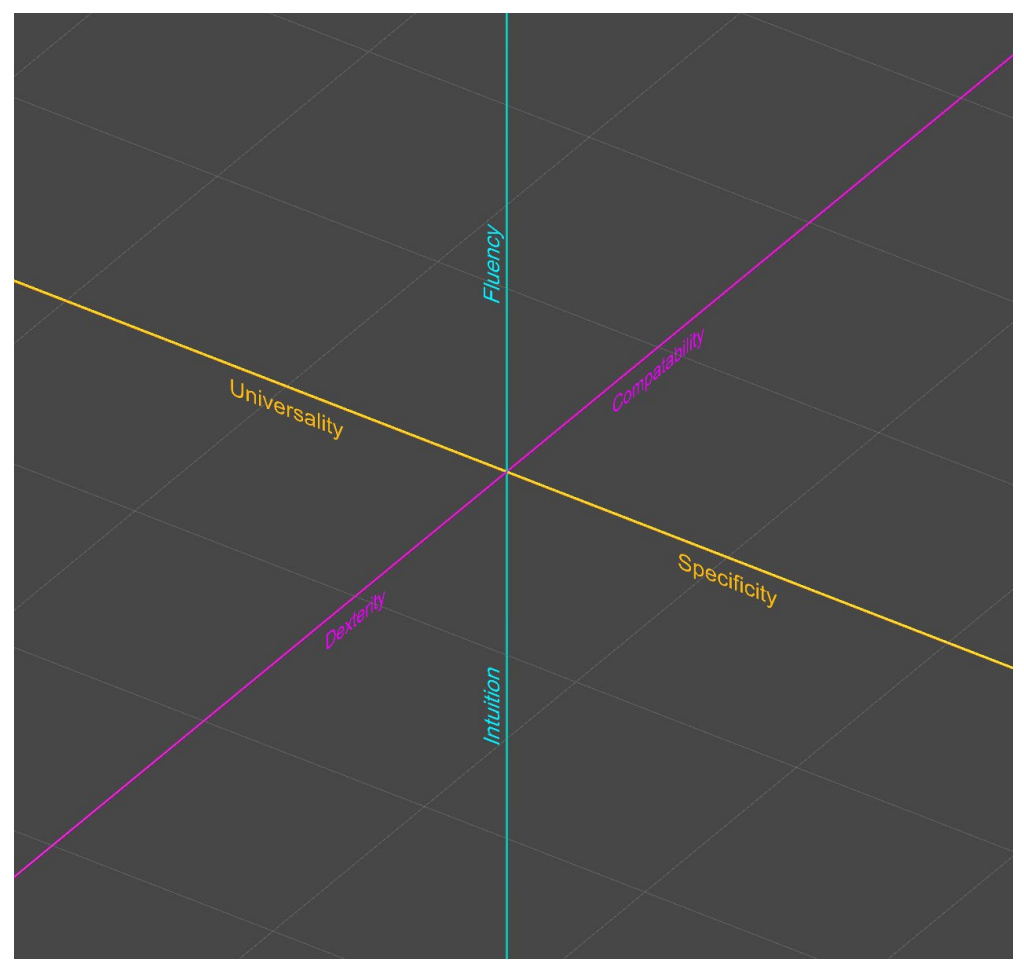

Figure 15: The Convergence of Touching Light in the User-Platform-Compiler Volume

While the decisions may seem simple (and if the goal is to recreate the Touching Light experience that I have developed for the HoloLens 2, then perhaps in many ways they are), these three components provide a foundation for the sorts of critical considerations that will inform any music-making in Mixed Reality, Touching Light or otherwise. Furthermore, while these three components form a substantial core of the process for making music in Mixed Reality, their consideration is not necessarily exclusive to MR settings. Any musical performance will feature 
at least one 'user!' And while the skill sets for a traditional performance and an MR performance may differ, many necessary qualities will remain the same.

\section{A Translucent Performance}

The second frame within the larger Invisible Art framework looks at MR music-making through the lens of musical performance, specifically by orienting six musical elements along the three well known dimensions that govern our physical world: length, height, and depth. Primarily these three dimensions are meant to be used as a mnemonic device to consider the six elements that are associated with them.

Unlike the 'critical components' of user, platform, and compiler, the dimensions that we will consider are not black and white barriers to entry into MR music-making. But while these elements are not necessarily equally important in every performance, as with so many aspects of music-making in general, a greater level of intentional care directed toward any of these dimensions will only deepen the potential for musical expression in Mixed Reality.

Already present in every live musical performance, these dimensions provide unique insight within the context of MR music-making. In the physical dimension, many of these considerations will not be so different from those that musicians, particularly percussionists, already navigate in their physical performances. But just like Mixed Reality as a medium, it is the integration of the virtual element with the physical element, as we will see, that fundamentally augments the meaningfulness of these decisions. By more fully considering the ways each of these dimensions are presented in MR, a level of translucence may be achieved, allowing the audience to more fully 'see' the musical elements at work.

\section{Page $\mid 73$}




\section{The X-axis: Length (As 'Time' and 'Size')}

Just as 'volume' has come to mean different things depending on the context, so too does 'length' measure both time and space. When considering the duration of a performance there are several concepts that may be uniquely suited to specifically Mixed Reality performances. In many ways, duration may be determined by the same sorts of decisions that any musical performance is governed by, and so it is important to investigate specific elements of MR musicmaking that are not necessarily present in traditional performances. One of the biggest limitations for the duration of MR music at the current time is battery life. In the case of the HoloLens 2 ("HL2"), if the battery dies, the MR component of the performance immediately ends, as it will with any untethered MR device. As battery technology continues to advance ${ }^{47}$, this initial consideration may become obsolete, but at the current juncture, is a necessary factor when developing performances in MR spaces.

Additionally, while not unique to MR music-making, it is always important to be aware that many of the interactions and experiences presented to the audience during a performance will be new to them, and so care should be given when considering duration and its relation to this novelty. Guiding questions may include:

- How long will the battery last; is it affected by the intensity of the MR program?

- Who is my audience; are they new to MR?

- If so, what percentage of the performance should be 'new' to them?

\footnotetext{
${ }^{47}$ This barrier is, of course, easily overcome with an external battery pack of sufficient wattage which would allow the HoloLens to maintain a positive charge rate throughout the performance, effectively eliminating the battery life from considerations of duration.

Page $\mid 74$
} 
- Are there interactions that will fatigue the performer?

- If so, how long can they physically maintain the performance?

- More abstractly, to what lengths must the audience go to understand the music?

By asking these questions we have an opportunity to think more critically about the musical elements of an MR performance, and thus an opportunity to make more and better music.

Conceptually, 'length' can also refer to 'space' or 'size.' One of the main considerations when performing music in Mixed Reality is how much 'space' the virtual elements of the performance occupy. While the HoloLens 2 is limited in the amount of space that it can 'see' at any one time, the device can hold a much larger volume of contiguous space in its memory, allowing holographic objects placed in a physical location outside of the current mapped radius (even allowing for objects to be placed in other rooms) to be 'remembered' when re-entering that space. If a work requires the use of a larger than $10 \mathrm{~m}^{3}$ volume, a method for placing objects in their necessary locations should be considered for the performance.

Touching Light necessarily exists within the hardware limitations of the HoloLens 2, but the nature of the work is such that all the assets, instructions, and interactions are translatable and transcribable to other platforms with a minimum amount of effort. 


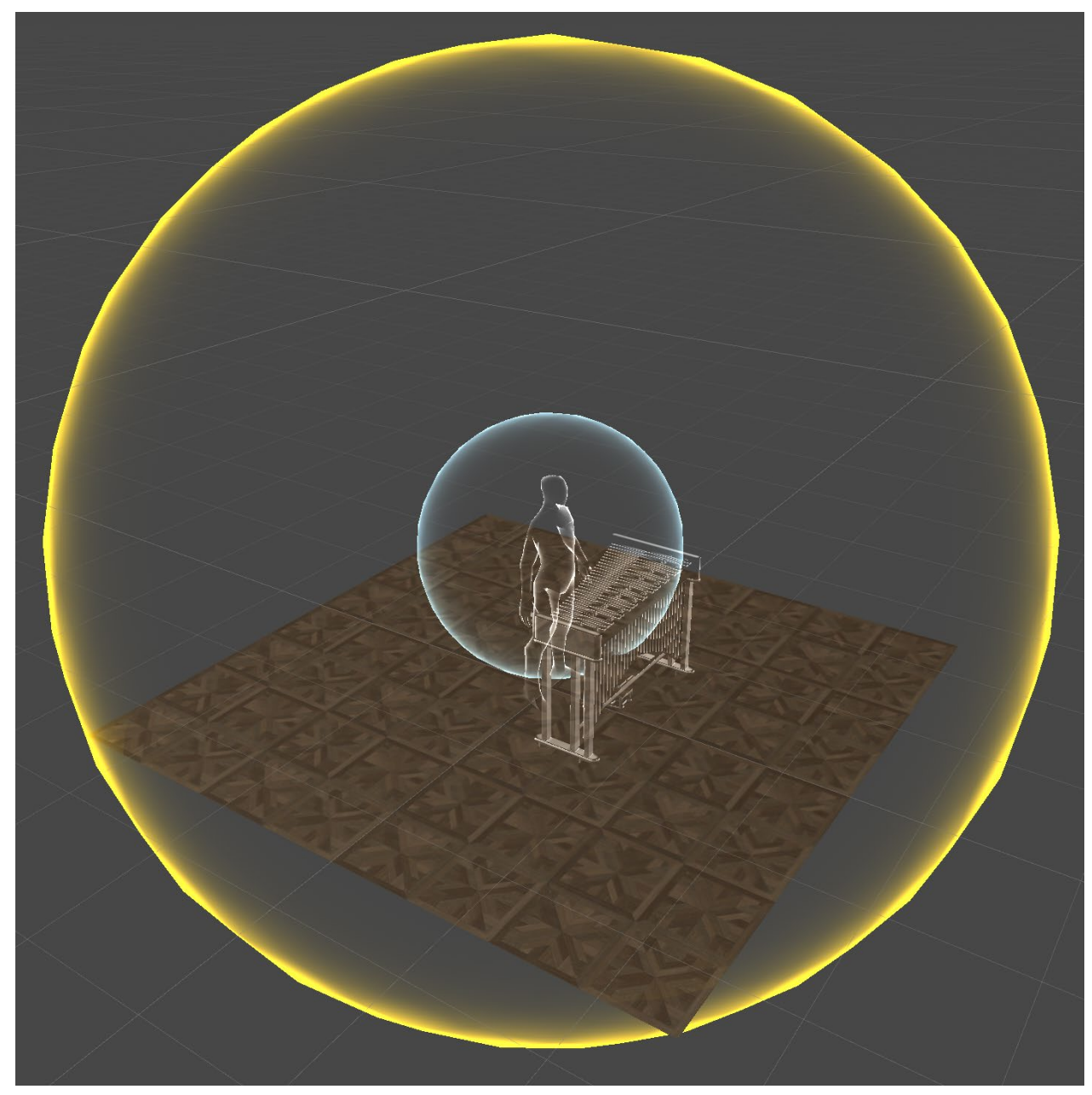

Figure 16: Representation of Near-field (blue) v. Room-scale (yellow) volumes

The 'room-scale' design of the work expects that the spatial dimensions of a performance should not exceed approximately twenty feet in any dimension in a radius around the user. Naturally, this is well within the typical boundaries necessitated by most physical interactions.

While Touching Light is designed with a typical recital hall in mind, by considering 'space' as a musical dimension, it is easy to see how the same sorts of interactions might be scaled up or down to accommodate different venues. Not unlike the way that a musician will adjust their sound to the 'room' they are playing in, so too may the MR elements within a performance be adjusted to better serve the space within which they are being performed. Instead of increasing 
dynamics in a larger hall, an increase in the scale of gestures may be appropriate; inversely, akin to performing with a more intimate sound in a smaller venue, more rapid and smaller gestures might be used in closer quarters. In considering 'space' as a musical element, we might ask:

- How large is the hall, and what MR elements are most easily scalable?

- $\quad$ If I am using MR instruments, will I need some sort of sound reinforcement?

- How overt should gestural interactions be? Do I need to telegraph?

- How much physical area do I have to work with on stage?

- Are there other MR performers? Other MR observers?

\section{The Y-axis: Height (As 'Verticality' and 'Climax')}

Continuing with our three-dimensional model, 'height' presents itself in both concrete and abstract ways: to determine the necessary verticality and to designate the conceptual climax of a musical work. Height, for non-percussionists, may be of little consequence in their musicmaking. For the percussionist however, the concept of 'height' is often one of the first things considered: is the instrument at the correct height to provide the optimal playing surface; do the heights of different instruments vary significantly, and if so, will that help or hinder accuracy and expression; will the height of various barriers (instruments, music stands, trap tables) interfere with the visual element of the performance?

While MR environments engage height differently than purely physical ones, verticality is still a necessary consideration. By default, Unity assumes that a basic object will inhabit approximately 1 cubic meter of physical space; while not all objects follow this principle, this fundamental conception helps to configure the objects and elements that were designed for Touching Light in two primary ways. 
The first of these elements is scalability: every object or collection of objects present in Touching Light is intentionally oriented in a way that makes resizing elements not only possible, but intuitive. In the first movement of Touching Light, the user can drastically alter the size of the 'mixer.' How they choose to alter the scale of the console may depend on any number of factors, including the distance at which they desire to interact with the virtual object (by scaling the object smaller, the user may find it easier to interact with it at a closer distance, etc.), or perhaps the desired verticality of their physical gesture in relation to the virtual object (by scaling the object larger, the user may find there the large physical gesture required to interact at this scale is more evocative of the sweeping motions that hope to communicate).
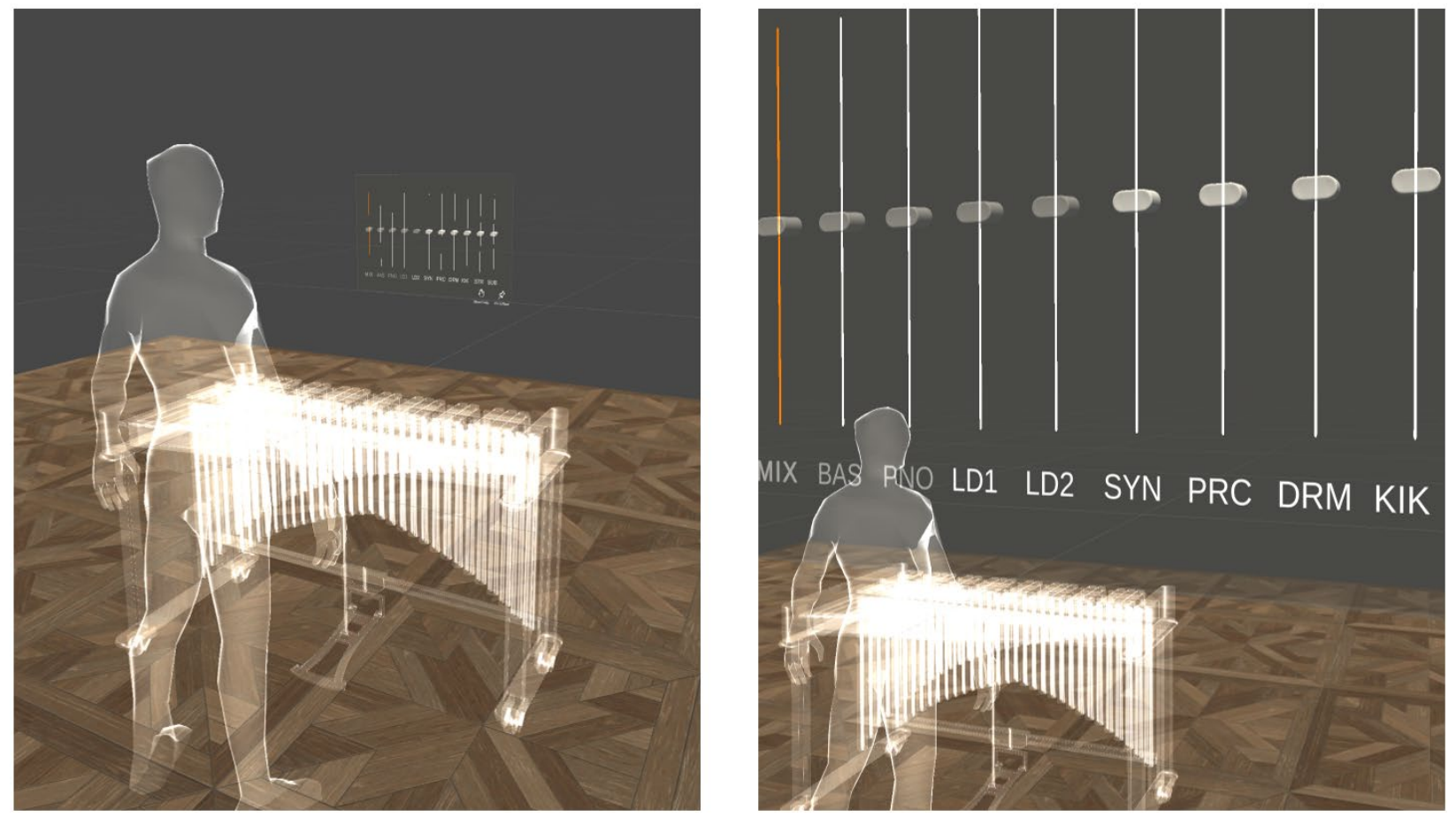

Figure 17: A Rather Small (and A Quite Large) Virtual Mixer Console

For the audience, it is necessary to consider the size and expressiveness of your interactions within the virtual environment, and how they will communicate to those who may or may not be familiar with the medium. It is natural to develop gestural and focal shortcuts, saving time and Page $\mid 78$ 
energy by exerting the smallest possible physical motion to achieve the same result. While these practices have a place in MR music-making, it is important to consider how often gestures need to be exaggerated in order to communicate effectively to an audience. Try to think about the performer's gesture from the audience's point-of-view:

- Which motions will communicate clearly regardless of scale?

- Do some gestures need to be larger, smaller?

- Even without seeing the virtual object, is it clear what the performer is doing?

- Is there a better, more communicable gesture?

Without an intentional investment in the shaping of a musical narrative, an audience is unlikely to engage as fully as when the narrative, and its subsequent storytelling climax, are clearly presented. This can be achieved through any combination of means, but it is these touchstones of familiarity, these standard musical practices that can provide access to even the most esoteric performances. Similarly, considerations of presentational climax should be considered when performing music with MR elements. A consideration of accessibility to performances of MR music is the most necessary to consider, as an audience who does not have access to the virtual volume in some way will have a truncated experience of music in mixed reality ${ }^{48}$.

\footnotetext{
${ }^{48}$ I use the term 'truncated' here with an underlying assumption that the goal of a MR performance would be to engage with the audience in both a physical and virtual volume, but I recognize this may not be the case in every scenario, or even always the ideal scenario. While detractors may consider there to be little value to observing an individual interacting via unknown gestures with an unseen world, viewed through the lens of dance and movement, even the gestures themselves can imply interactions with the virtual volume in ways that are auditorily accessible.
} 
Touching Light is intended to be performed in tandem with a live stream from the HoloLens 2 to a computer or other device connected via a high-speed Wi-Fi network to a large screen or other audience-facing display. This sort of projecting allows the audience to engage with the virtual environment in a visible way, pairing the complexity of gestural MR interaction to their understanding of the musical elements engaged throughout the work. This dissolution of the supposed barrier of an imagined 'fourth wall' may in fact heighten the audience experience, furthering their engagement with the mixing of realities unfolding before them. Consider,

- What will be new to the audience?

- What other context may inform their understanding?

- How can these elements be combined to create something accessible?

- What is the sustainable height of MR elements within the volume?

- How can you present this to an audience that can't engage with the MR space?

\section{The Z-axis: Depth (As 'Density' and 'Attention')}

Depth can describe a physical measurement, density, as much as a virtual dimension, obfuscation, and both degrees of consideration may help to contextualize considerations of this axis when making music in Mixed Reality.

Conceptions of physical space and orientation are fundamental to the musical and logistical practices of the percussionist. Without understanding and consideration given to both length and height, it quickly becomes difficult to present any sort of musical performance that engages with an audience in a meaningful way, let alone one that navigates a collection of instruments in an efficient and expert manner. Yet this final dimension of depth will often make or break a musical performance. In many ways, the draw of the traditional percussion ensemble concert is its density, the sheer number of instruments, styles, textures, timbres, and techniques Page $\mid 80$ 
on display. The percussionist must be a jack-of-all-trades, and this level of complexity is compelling to many audiences.

Yet there is, of course, a breaking point. Even given an infinitely large stage, with an infinitely ideal view for every member of the audience, there is a line past which accessibility and engagement begin to rapidly degrade; it is possible to do too much.

Touching Light is designed for the specific combination of percussionist and Mixed Reality Environment, and as such, imposes intentional limits on the density of both interactions and objects on stage within the MR environment at any one time. I have carefully considered ways in which the density of the virtual environment compounds with the density of the physical environment. In discerning an appropriate balance, I follow this principle: 'you can't fill the same space twice.'

This may seem a little too obvious, but when imaging the sort of possibilities present in an MR environment, I could imagine how holograms would unintentionally end up sharing the same space with physical objects, and vice-versa. To that end, the overall level of component density (virtual OR physical objects in their respective environments) is relatively low: the total number of physical and virtual objects combined in Touching Light is less than some traditional percussion ensemble concerts I have attended...!

We can see how, when presenting MR music, this dimension of density is doubly important. Not only do we ask an audience to be open to an experience that may be largely novel, but simultaneously, to engage with musical performance in a shared, Mixed Reality generates a compounding load of conceptual density. Each individual element present, without proper context and introduction, may remain largely unintelligible to audiences that do not have prior 
experience with MR environments. When discerning the density of any MR space, it may be helpful to ask questions like,

- How would I fill this space if I only have physical objects? Virtual ones?

- Does the stage feel cramped? Can I move about freely?

- How many virtual objects can I see (and thus render) at a time?

- How many things will the audience need to keep track of?

- $\quad$ Can I do more with less? Am I doing less with more?

Depth is as much a virtual dimension as it is a physical one, encompassing considerations ranging from emotive force, compositional quality, cultural impact, or historical relevance, etc. Yet there is a further contextualization to the dimension of depth as a virtual construct (that is, one which is observed outside of a physical space): one of attention. With such an expansive palette of instruments at the percussionist's disposal, not to mention the incredible versatility in terms of tone, texture, and timbre that each instrument presents, it is not uncommon for the percussionist to face this question of focus: "Which of these instruments, sounds, timbres are the most important? What should have my attention? And inversely, what should be given our foremost attention?"

This consideration presents itself in different ways depending on the context. In practicing percussion, we ask: which of these instruments should I devote my time to; what do I choose to practice; where do I invest the limited number of hours in my day? In rehearsal preparation: what do I need to trust and what do I need to prepare; do I need to work on the passage that is full of arpeggios, or should I focus my attention on the section with a bunch of disparate rhythms, or where I am exposed, or where I have the solo? In ensemble performance: when should I bring my sound to the forefront, and when should I exist as a supporting voice; Page $\mid 82$ 
when should I fade into the background? Each of these considerations can lead to a variety of conclusions, and an even greater diversity of decisions and technical executions, yet each deal with the fundamental concept of attention: what needs to be in focus, and what does not?

In preparing works that include MR elements, attention is a physical consideration regarding the visibility of holographic objects and the ways that user gaze can be tracked as an interactive parameter but is also a much more fundamental consideration relating to intention and importance. This dimensional model assumes that MR elements are being deployed in a virtual volume (e.g., holograms, gaze tracking, gestural interactions, etc.) in conjunction with elements in a physical volume (e.g., a stage, physical instruments, lighting, etc.). In this scenario, it is important to determine early on what the 'focus' of the performance or experience will be, and by extension, where you desire to direct the audience's attention. The following considerations may help to guide us:

- Does one reality (physical or virtual) require more attention?

- How will the audience know when something is important?

- If an element is 'not as important,' what function does it serve? Is it needed?

- Can I see what I need to see when I need to see it?

- What is the most important?

\section{Dimensionality as a Structure}

In the immediate future I expect that for most audiences, the novelty of MR musicmaking will generate a significant obfuscation to any traditional and physical elements involved in the performance. Therefore, a greater weight and attention must be given to the physical volume in order to offset the bias towards novelty! Yet of course, this consideration however must be tempered by a recognition of the fleetingness of that self-same novelty. While the idea Page | 83 
of 'touching light' may be initially intriguing, if the virtual volume is underdeveloped, if it is not given appropriate consideration and care, then when the initial sheen of a novelty inevitably wears off, the remaining performance may not hold the audience's attention for long.

This structure, derived from these dimensional forms, is meant to provide a baseline for the development of the musical Mixed Reality environment. The technical wonder of 'touching light,' must be underpinned by the rich traditions of musical practice in the physical environment, so that at the marriage of these two spaces, a new artistic medium can be realized: one that draws upon both the physical and virtual, arranged in these triadic dimensions to conjure a new and exciting harmony.

As with any new venture, we must consider the purpose: do we seek to present the archetype, or the prototype, the culmination or the precipitation, the end or the beginning? This model aims to be flexible and useful at any point along that continuum, but special care and focus here is given to opening the way to music-making in Mixed Reality. In total, as we consider music-making in Mixed Reality, these questions (collected from those presented throughout this section) may serve to guide our efforts.

In considering 'length:'

- How long will the battery last; is it affected by the intensity of the MR program?

- Who is my audience; are they new to MR?

- If so, what percentage of the performance should be 'new' to them?

- Are there interactions that will fatigue the performer?

- If so, how long can they physically maintain the performance?

- More abstractly, to what lengths must the audience go to understand the music?

- How large is the hall, and what MR elements are most easily scalable?

- If I am using MR instruments, will I need some sort of sound reinforcement? 
- How overt should gestural interactions be? Do I need to telegraph?

- How much physical area do I have to work with on stage?

- Are there other MR performers? Other MR observers?

In considering 'height:'

- Which motions will communicate clearly regardless of scale?

- Do some gestures need to be larger, smaller?

- Even without seeing the virtual object, is it clear what the performer is doing?

- Is there a better, more communicable gesture?

- What will be new to the audience?

- What other context may inform their understanding?

- How can these elements be combined to create something accessible?

- What is the sustainable height of MR elements within the volume?

In considering 'depth:'

- How can you present this to an audience that can't engage with the MR space?

- How would I fill this space if I only have physical objects? Virtual ones?

- Does the stage feel cramped? Can I move about freely?

- How many virtual objects can I see (and thus render) at a time?

- How many things will the audience need to keep track of?

- Can I do more with less? Am I doing less with more?

- Does one reality (physical or virtual) require more attention?

- How will the audience know when something is obfuscated?

- If an element is 'not as important,' what function does it serve? Is it needed?

- Can I see what I need to see when I need to see it?

- What is the most important? 


\section{Mixed Reality as a Liminal Space (an Allegory)}

Mixed Reality exists as a merging of the physical and virtual worlds, giving us access to the best (and sometimes the worst...) of both. There is often something 'magical' about experiencing Mixed Reality for the first time; the unique intersection of imagination and interaction, of light and touch is compelling. When we think about Mixed Reality as an artistic medium, we can begin to see how a deeper understanding of precisely where and how these two worlds blend together can help us to explore and discover.

Because Mixed Reality focuses on a sort of 'liminal space,' a boundary zone between the physical world and the virtual world, when we engage artistically in this area, we should first understand two things. Firstly, in order to engage with objects and environments in Mixed Reality to their fullest potential, we must understand from which of the worlds these objects originate: the physical or the virtual?

Objects from the physical world will imply certain types of interactions, while objects from the virtual world will necessarily not imply those sorts of interactions. If you imagine that there exists an MR stone, made of light, but otherwise indistinguishable from a physical stone, we will assume certain things about how it should behave, and by extension, how we can interact with it. These assumptions are based, in part, on the fact that we know that the stone 'came' from the physical world, and so should have physical properties; we assume that it is tangible.

The second part of this equation is that we must understand at what level the object has passed into Mixed Reality: is the object meant to be a concrete, one-to-one representation, and if so, what rules then must this object follow based on its origin; is the object meant to be an abstraction, a generalization of an object, and if so, what rules might this object break, since it is 
not concrete; or finally, is this an esoteric object, a container into which meaning is yet to be placed, and if so, how should we determine its meaning?

If our stone is meant to represent a real, literal stone in the physical world, one that someone has really seen, handled, and studied, then we understand that our stone, this MR object, should behave like that stone; because it should be the same as an actual object, it is a concrete representation (no pun intended). By combining our understanding of our stone's tangible and concrete properties, we can determine our stone's liminality, or 'where our assumptions about our stone intersect with the MR environment.'

Now, imagine if that same stone, our stone, wasn't actually based on a singular, specific stone, but more modeled after the 'idea' of stones; maybe our stone isn't photorealistic, or maybe it's got some cool colors on it that mean that it's probably just meant to represent a stone when we interact with it. Because we know that our stone isn't that stone, we don't expect it to act like that stone. In fact, we might try to do things with our stone that we wouldn't do if it were supposed to be that stone exactly; this change in our assumptions about our stone are because we understand that now it's an abstraction, a representation of a generic 'stone,' rather than an emulation of a specific stone.

But our stone is still a stone, after all, and so because we know that it's tangible, and so we assume that it has some properties of tangible objects like gravity, mass, volume, etc., we continue to make certain assumptions. If we were to drop our stone on our foot, even though we know that it is not physically there, my money says that we would be surprised that it didn't hurt (it still gets me from time to time!). Because we expect it to. Because it's still a tangible object. 
Let's imagine that our stone doesn't even look like a stone, but instead is just a vaguely stone-shaped ball of light. We can still recognize it as our stone, even though now it's clear that it's nothing like that stone anymore. But when we pick it up, what do we expect is going to happen? It doesn't look like a stone, but emotionally, conceptually, it still feels like a stone (it's our stone, after all!). This moment of dissonance occurs because our stone has become an esoteric object, one which retains the properties that it has because we, as an observer have decided that this ball of vaguely stone-shaped light is our stone.

While this metaphor may seem silly, I've found that the way that we think about MR objects, and specifically the assumptions that we make about their origins and their intersectionality with the MR environment inherently guide the ways that we attempt to interact with them. While there is nothing to stop us from acting against this intuition, by being aware of these assumptions I believe that we can be more intentional as well as more creative in the ways that we make music in Mixed Reality.

The different combinations of these two origins, the physical (tangible) world and the virtual (intangible) world with the three possible levels of representation (concrete, abstract, or esoteric) can help to identify presumptive properties of MR objects as soon as they enter the MR space.

By discerning what sorts of liminalities are being explored, exhibited, or employed throughout an environment, not only are we able to contextualize the appropriate expectations for how we might interact with these objects, but furthermore, generates creative opportunities to explore the boundaries of our assumptions through our performance. 
Like the area where the proverbial forest meets the field, this shared environment, this liminal space in nature accounts for some of the most abundant and varied biospheres; Mixed Reality is much the same way. It is in this shared space between the physical and the virtual, between 'reality' and 'imagination,' that creativity is most abundant.

\section{Hybrid/MR Interactions}

"When you change the way you see the world, you can change the world you see."49 These considerations bring us inevitably to the possibility of engaging with uniquely hybrid liminalities, begging the question: are there interactions that are unique to these MR spaces; is there a MR liminality ${ }^{50}$ Understanding the two obvious origins for MR objects (the physical world or the virtual world), we can begin to imagine how objects whose origin is the liminal space (of Mixed Reality) might behave.

\footnotetext{
49 Twitter. "Microsoft HoloLens on Twitter.” Accessed January 29, 2021. https://twitter.com/HoloLens/status/576531839856168960.
}

${ }^{50}$ At the current moment in time, our technological innovation in Mixed Reality has outpaced our understanding and exploration of the creative space. Microsoft has created a scenario with the HoloLens whereby there exists a sort of artistic gold rush, just waiting to happen, but the sorts of tools needed to pan for the 'gold' are currently still prohibitively expensive and seemingly difficult to learn.

While hybrid interactions are clearly the obvious next step in this series of leveled liminalities, it will be some time before these truly hybrid interactions become the focus of development with this technology. Current best practices, even among Microsoft engineers, largely involve interactions with liminalities that would be described as 'concrete' and 'tangible' by this nomenclature: in essence, porting 2-D screens into 3-D holographic space. 


\section{In/tangible Superpositions}

In quantum physics, superposition refers to the state of an object that is effectively in 'two places at once,' or in our case, an object that is from two places at once. Hybrid objects, or those which are in a MR superposition will have both tangible and intangible properties, creating unique interactions that are only possible with these sorts of 'hybrid' objects and environments. The final movement of Touching Light, Synecdoche, was specifically designed to take unique advantage of this hybrid superposition.

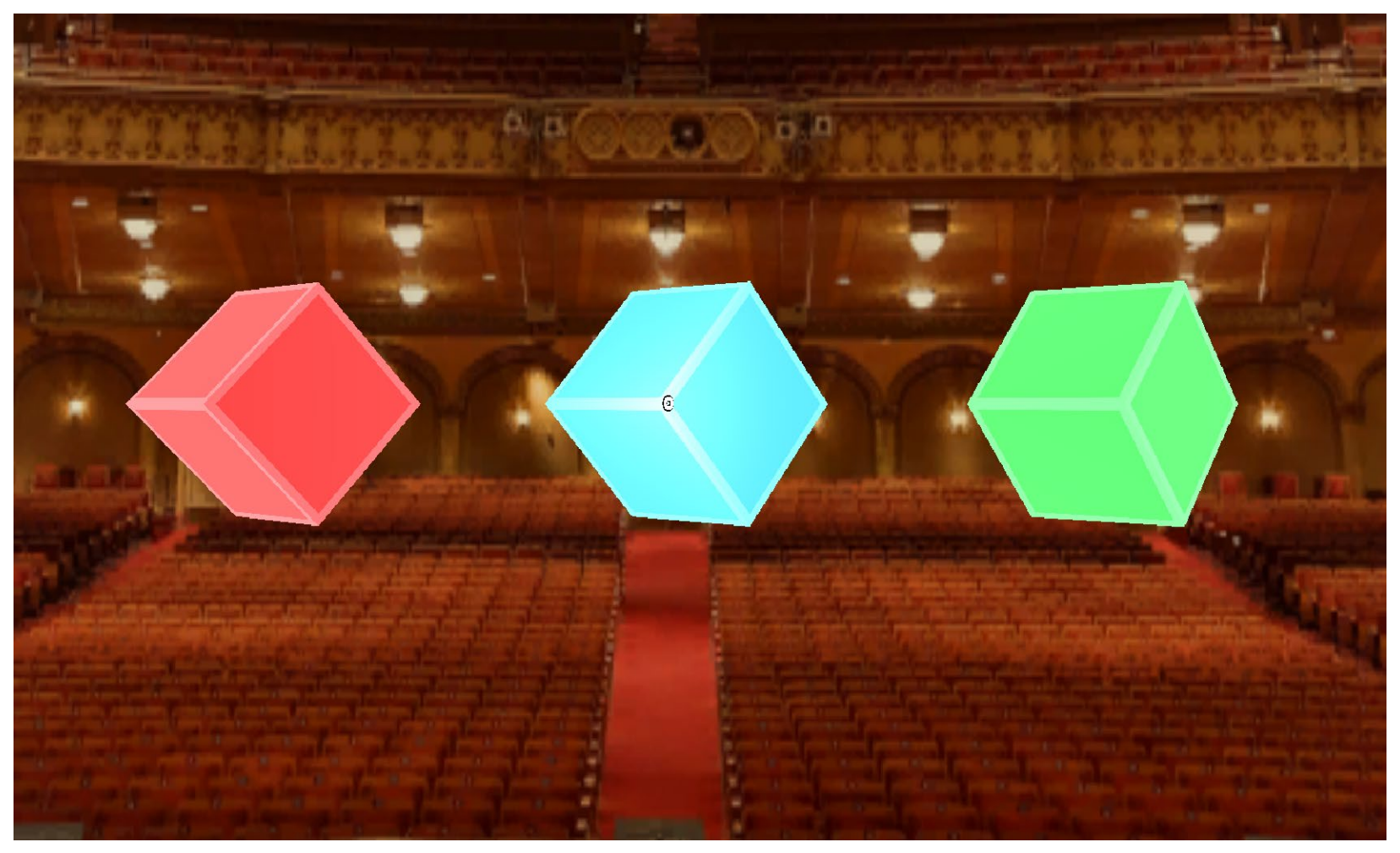

Figure 18: Three Cubes from Touching Light (iii. synecdoche)

The scene opens as any other but does not immediately create any virtual objects nor give any indication, beyond its nonresponse, that the piece has begun; it is up to the performer's discretion what should be rendered, and when. The first interaction available requires a foreknowledge on the part of the performer of the existence of an intangible esoteric menu, 
accessible by looking at the palm of the left hand, at which time a particularly unmusical menu will appear: "Freeze all, Reset, Unmute, Generate Red, Freeze Red, Mute Red, Generate Blue, Freeze Blue, Mute Blue, Generate Green, Freeze Green, Mute Green...,” etc.

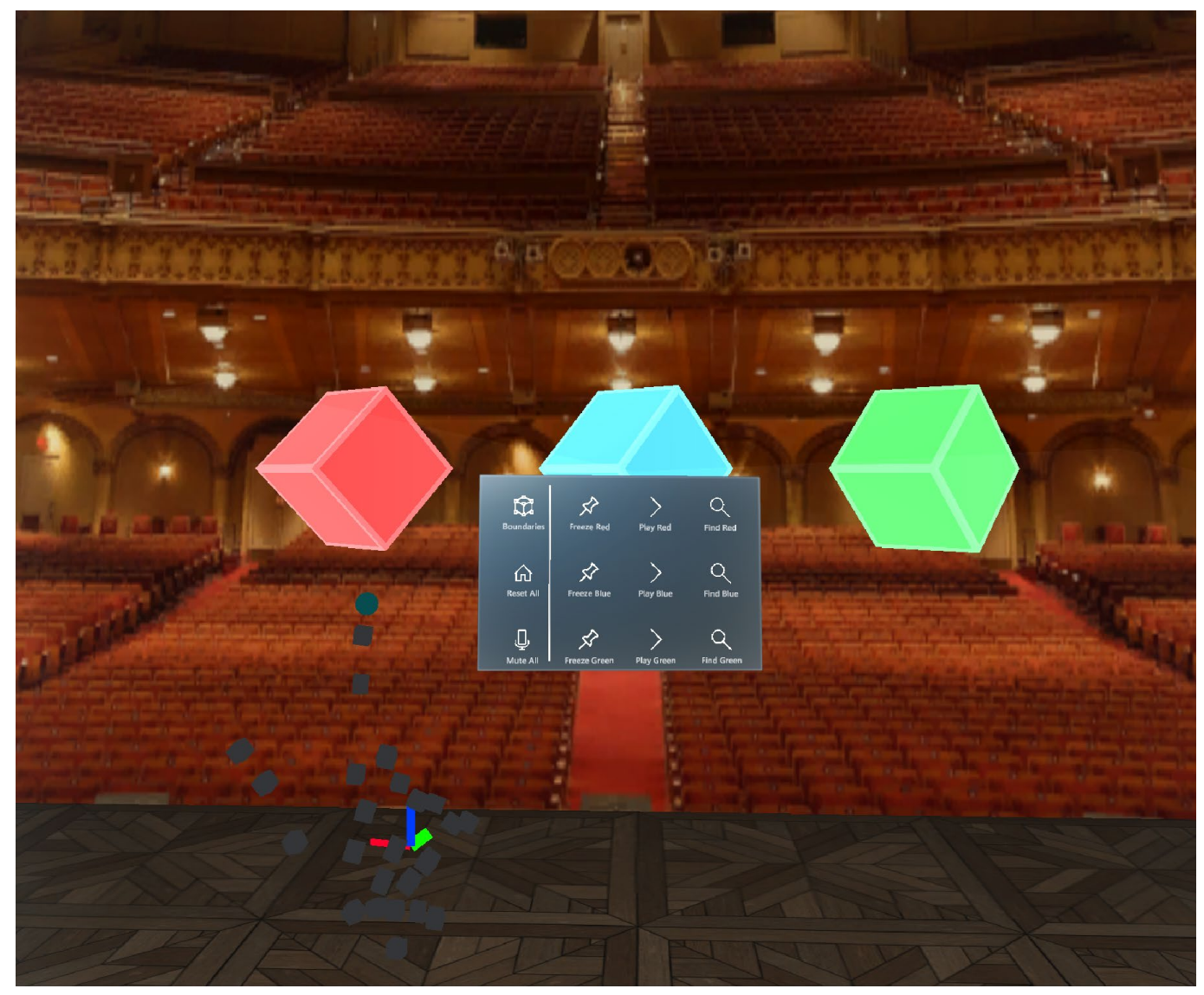

Figure 19: Contextual 'hand-menu' from Touching Light (iii. synecdoche)

The performer will, inevitably, choose to 'generate' one or more of the colors, at which point a simple (seemingly concrete-tangible) cube of the associated hue will appear to hover before them and, depending on their subsequent selections, might begin to 'sing!' This cube, along with its siblings, work in concert to create an abstracted-intangible audio-scape which features a combination of three interwoven musical elements.

As the three cubes hover there in mid-air, the audience and the performer alike will naturally begin to wonder as to the nature of their interactions; so far, we have seen a virtual Page $\mid 91$ 
mixer hang in open air, be resized, be set on all manner of different physical surfaces, and a collection of abstract images rotate in a sort of carousel of memory around the performer - what do these cubes $d o$ ?

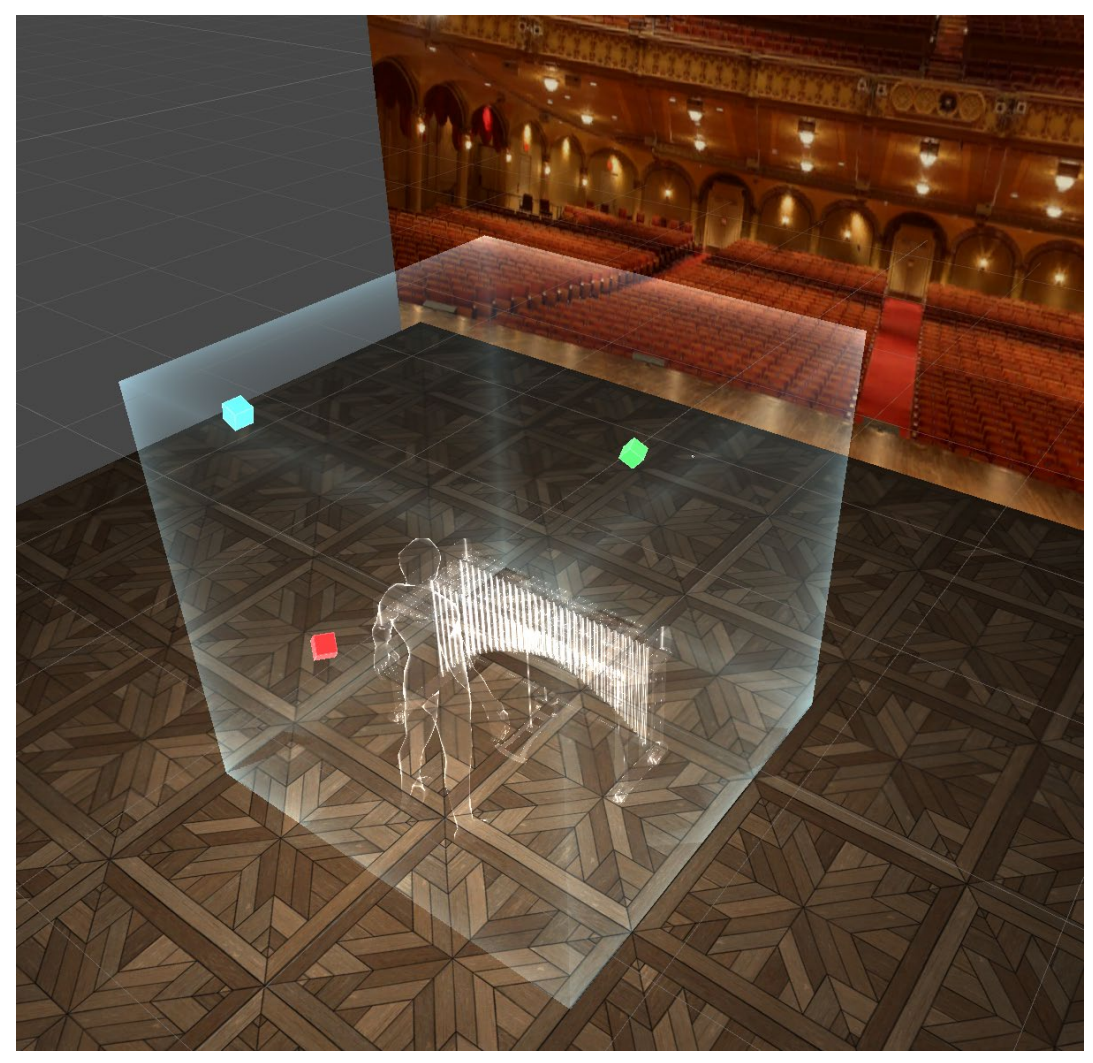

Figure 20: Representation of Cubes in Motion from Touching Light (iii. synecdoche)

At this point, the audience may have noticed the very edges of audio spatialization, one of the tenets of a hybrid liminality: the audio created by these 'concrete' objects seems to know where they are in space; as the performer moves away from them, they grow quieter, if the performer circles them, they seem to be heard from the correct direction, and if the performer touches them... they move, weightlessly. Not like the mixer, but like they are tiny music-making satellites in outer space, untethered by gravity, but existing onstage with the performer. And as they float, they collide, and as they collide, they ricochet, and as they ricochet, they rebound 
floor-wards, and when they strike the floor (and they, the holographic, virtual objects $d o$ strike the physical, real-world floor) they bounce off that as well, twisting, and turning, and real. Suddenly, this hybrid liminality is no longer a theoretical model, but a proper 'mixed reality.'

\section{A Framework for Music-making in Mixed Reality}

In this chapter I have considered the findings generated by the development of Touching Light, a three-part framework through which current and future works for MR music-making may be oriented. By understanding the three critical components to a digital MR score, we can more appropriately engage in preparing opportunities to perform music in Mixed Reality. By examining musical elements through the mnemonic of three dimensions, we can work to make our performance translucent, presenting a deep intentionality in our desire to connect with our audiences. And finally, by considering the path by which an object enters Mixed Reality, we can begin to understand the sorts of assumptions that are inherent to performing within the MR medium, and in that understanding, explore even more liminal possibilities. 


\section{Chapter 5 - Only the Beginning}

In his 1997 article for the Cambridge Opera Journal titled "Technology of the Archaic: Wish Images and Phantasmagoria in Wagner," ${ }^{51}$ musicologist Alastair Williams considers the tensions of tradition and modernity in Richard Wagner's 1876 Der Ring des Nibelungen, specifically in the characterization of Brünnhilde, who,

...would envisage neither a hydraulic model of revolution whereby a new society miraculously emerges fully formed as the old withers, nor abandonment to fate; she might instead seek to release unrealised [sic] social forms embedded in the past and present. ${ }^{52}$ Williams, alongside Benjamin, Adorno, Abbate ${ }^{53}$, among others engage with these tensions present in the work of Wagner, specifically, yet it is not difficult to expand these inherent elements to contextualize a discourse of art, Wagnerian or otherwise: there are often two paths laid before us, one of which calls for the dissolution of that which came before in favor of modernity, the other to reject that which lies ahead in favor of tradition - but like Wagner's Brünnhilde, there is almost certainly a third path which celebrates both the new and the old, the modern and the traditional.

As I have explored the unique potential of Mixed Reality as a medium for music-making, there have been no shortage of examples to demonstrate the unique sorts of artistic interactions that can be borne of the liminal space between physical and virtual environments. It is like Williams says of Wagner's Brünnhilde: Mixed Reality music-making ‘does not envision a

\footnotetext{
${ }^{51}$ Alastair Williams, "Technology of the Archaic".

${ }^{52}$ Ibid., 74.

${ }^{53}$ Walter Benjamin (1892-1940), Theodor Adorno (1903-1969), and Carolyn Abbate (b. 1956) are musicologists and Wagner scholars whose critical theories are still considered impactful in the $21^{\text {st }}$ century. 
hydraulic model, but instead seeks to realize forms embedded in the past and the present;' and perhaps even those embedded in the future.

\section{Findings}

The unique opportunity to engage music-making and Mixed Reality is not something that I take lightly; what began as a pipe dream just over a year ago has had a significant impact on the ways that I engage with both music and technology. Through this process of discovery, I have consolidated my findings into three main categories: those that deal directly with music-making as a performing art, those that engage music-making as a pedagogical discipline, and those that position music-making as a shared cultural experience.

The principal findings of this project are the components of the framework presented in Chapter 4. It was through the process of developing Touching Light that I was both able to confirm my initial suspicions regarding the potential for the combination of Mixed Reality and music-making, and furthermore, develop the three different postures or 'frames' through which to engage with MR music-making in the future.

More broadly, I was pleasantly surprised to discover that Mixed Reality is a deeply creative medium, and as such, engages easily with the process of music-making. From the deeply satisfying manipulation of a standing wave through the miniscule gestures of a rotating hand, to the shocking immersion of a massive holographic carousel slowly rotating around you while you play, there is something much more connective about the spatial interactions presented by MR than the limitations of peripherals like a mouse and keyboard to control those same musical and visual elements. 
Concurrent with these findings were some small 'surprises.' These ranged in significance from simple assumptions that I had taken for granted regarding the nature of sound and instrumentation, to significant shifts in technologies during the research process, including no small number of skill barriers due to my limited experience with programming in general, and developing and MR technologies specifically. While these moments were undoubtedly challenging at the time, as with any worthwhile endeavors, by finding a way to navigate these issues, I became increasingly more adept at lessening the impact of those issues that I would face in the future. Furthermore, some of these missteps provided opportunity for me to engage with musical and technological online communities who were always willing to help.

\section{Making Music in Mixed Reality (How to Get Started, and Why You Should)}

Already, so much of what we do as musicians is, within the context of society at large, a niche endeavor; for the percussionist, these degrees of separation can seem even more severe. But in the same ways that we as artists commit ourselves to the craft of music, and the practice of music-making, engaging with MR has only served to deepen those sorts of commitments for me.

\section{For Musicians or ("Performers")}

For those individuals who are interested in the musical side of Mixed Reality, the first step to get your hands on a platform. Touching Light is obviously designed with the Microsoft HoloLens 2 in mind, but similar functionality is available through any number of other VR headsets. Once you have a platform, you will need to decide what you will perform. If you are working with the Microsoft HoloLens 2, a great place to start is with Touching Light! You can download the complete Unity file package from the link included in Appendix C. Follow the 
instructions from the Microsoft Mixed Reality Documentation, ${ }^{54}$ beginning at " 1 . Build the Unity Project." Once you have deployed the application to your HoloLens 2, load up the application, and explore!

One of the most profound discoveries that I have made while working with this technology is just how musical it can be. There is something about engaging with technology within the Mixed Reality volume, about 'spatial computing,' as Microsoft calls it, that seems intuitive and artistic. This simple fact has even more deeply convinced me that music-making in Mixed Reality is not just an interesting possibility, but a deeply meaningful inevitability.

\section{For Programmers (or "Composers")}

For those individuals who may be more interested in the nuts-and-bolts of developing musical applications for Mixed Reality, the first step is to familiarize yourself with a compiler. If you are interested in programming for the Microsoft HoloLens 2, the de facto solution at present is the Unity Development Engine, though support for other compilers is becoming increasingly available. You can download Unity Hub for free from their website ${ }^{55}$, and then following the instructions in the Microsoft Mixed Reality Documentation, ${ }^{56}$ beginning at " 1 . Introduction to the MRTK tutorials," you can begin to develop your first Mixed Reality application.

I would strongly advise that, once you get a handle on the basic functionality of the compiler and complete some of the beginning MRTK tutorials, take some time to consider what sorts of functionality you would like your application to demonstrate, the connect with the

\footnotetext{
${ }^{54}$ https://docs.microsoft.com/en-us/windows/mixed-reality/develop/unity/tutorials/mr-learning-base02?tabs=winxr\#1-build-the-unity-project

${ }^{55} \mathrm{https}$ ://store.unity.com/download

${ }^{56} \mathrm{https} / / /$ docs.microsoft.com/en-us/windows/mixed-reality/develop/unity/tutorials/mr-learning-base-

$\underline{02 \text { ?tabs=winxr\#1-build-the-unity-project }}$

Page $\mid 97$
} 
Microsoft MR community (via $\underline{\text { Slack }}^{57}$ or the Microsoft MR Tech Community forums ${ }^{58}$ ) and connect with other who may be able to answer your questions, and even help you with your project design.

Throughout the development process of Touching Light, I was surprised at not only how easy it was to onboard myself to Mixed Reality development by using the MRTK, but also by how friendly and helpful the then-current MR development community was. Whenever I had a question, or was struggling with some element of implementation, I would quickly be directed to the relevant documentation, YouTube video, or other resource that very often addressed the exact issue I was having without ever need to post snippets of code or consult more directly with someone on the project. As a bonus, I was also able to connect with a handful of individual who had a particular interest in developing creative applications for the HoloLens 2.

Mixed Reality, fundamentally, asks us to see the world differently, something that is so akin to the ways that as performers, we ask our audiences not just to hear, but to listen. By drawing the attention of those around us to something that we believe to be compelling, and even more when we can share something that we have had a hand in creating, we access a unique moment, a shared imaginative space and, in my experience, this is just the sort of thing that users of Mixed Reality are hoping to find. This project seeks to not only provide a philosophical understanding of Mixed Reality music-making, but also provide resources for those who are interested in exploring this artistical and technological medium.

\footnotetext{
${ }^{57} \mathrm{https}: / /$ holodevelopers.slack.com

58 https://techcommunity.microsoft.com/t5/mixed-reality/ct-p/MicrosoftMixedReality

Page $\mid 98$
} 


\section{Areas for Further Research}

This addendum will suggest large-scale applications and implementations of this specific technology, as well as the sort of lessons that it can teach in a purely physical environment, including reflections on the process of this research and a collection of topical summaries in areas for future research. Examples of some of these topics include navigating the performative experience and generating audience engagement in an unseen world, considerations of diversity in 'connected' and asymmetric performances, and considerations surrounding the presentation of digital solutions to analog problems. Mixed Reality provides a unique opportunity for the musician to engage with physical and virtual spaces, and similarly physical and virtual audiences; a great deal of opportunity exists in further study of this space.

\section{Live Concert Attendance in MR}

While companies like Wave $\mathrm{XR}^{59}$ are already engaging in wholly virtual musical performances with great success, further research is needed both in the areas of audience experience from virtual concert attendance, as well as in the areas of artist experience and engagement through wholly virtual medium. This of course says nothing of the wide range of topics that may be conceived of through a similar lens, but specifically for Mixed Reality performances and MR audiences, of which there are many, including but not limited to: simultaneous performances for physical and virtual audiences, performances featuring exclusively MR immersion, chamber ensemble performances within MR environments, etc.

\footnotetext{
${ }^{59} \mathrm{https://wavexr.com/}$
}

Page $\mid 99$ 


\section{MR Music Rehearsal Applications}

Whether for string quartet, drum-and-dance ensemble, or collaborative DJing, MR as a medium may provide unique vectors of engagement between members of groups as well as individuals willing to augment their artistic medium for the benefits granted by a Mixed Reality Environment. For the string quartet, a rehearsal wherein sheet music is projected holographically

rather than on physical music stands that obfuscate the bodies and instruments of the members of the ensemble may be used to increase connectedness and ensemble communication within the group. For drum and dance, there is an opportunity to supplement both the numbers of members in the ensemble, as well as the expertise of ensemble members by using virtual dancers or virtual drummers. MR may furthermore provide a collaborative environment within which to interact with other electronic musicians, regardless of location or distance.

\section{MR for Practice and Pedagogy}

Mixed Reality provides huge potential for individual practice and pedagogy, ranging from real-time hand tracking, visual and audio cues in conjunction with traditional notation, immediate visual feedback that responds to any number of discernable parameters, including intonation, rhythm, tempo, dynamics, velocity, etc. Furthermore, in conjunction with a physical playing surface of some kind (akin to a drum practice pad), a holographic overlay combined with real-time stick and mallet tracking (an extension of the native hand-tracking) could allow a student to practice on a virtual instrument that is visually and aurally identical to those on which they will perform, or even to practice on instruments that they would otherwise not have access to.

Applications also exist for other instrumentalists, dancers, conductors, visual artists, etc. to engage with life-size, representative, real-time, interactive models that can provide 
meaningful, qualitative feedback in response to the user's interaction; imagine a virtual ensemble that does not play the down beat until you conduct it, or a virtual dancer that does not move on to the next sequence of motions until you have matched their body position exactly.

\section{MR for Composers}

Mixed Reality also provides a new and exciting medium for composers who are looking to explore and engage with a wider range of possible gestural interactions and hybrid performance practices in their works. Conceptually, MR as a compositional medium divorces the compositional practice from the inherent necessity of physicality - should the performer's left elbow play the tam-tam? Do you wish that there existed an instrument that was both a marimba and a vibraphone at once? With a virtual environment existing simultaneously (and dimensionally) with the physical environment, these possibilities can now be realized.

\section{Conceptual and Philosophical Frameworks}

Vast opportunities exist when considering the sorts of foundational axes presented in this document and their application to any number of other practices and processes; a consideration of liminalities regarding the possibilities of audio processing in an MR environment, for instance, may provide critical guidance for best practices in the future, not only for live musicmaking, but for other creative, artistic, and commercial industries as well.

\section{Final Thoughts}

This research is only the beginning; I have endeavored to present a conceptual framework rather than an exhaustive analysis or an in-depth instructional manual with the intention that the ideas set forth in this document may be accessible, and more importantly, beneficial, to the greatest number of individuals. It is my sincere hope that this document will serve to orient, 
assist, and inspire musicians, artists, and audiences alike as we continue to navigate an increasingly digital and virtual existence. Perhaps more than any other time in history, only compounded by the incredible circumstances surrounding global health and the subsequent impact that a response to such scenarios require, we have been forced to think differently about technology, and for those of us who found ourselves suddenly unable to engage in live musical performances, neither as artists nor audiences, it is my conviction that mediums like Mixed Reality will only become more essential to exploring 'liveness' within the context of digital and virtual spaces. 


\section{Appendix A: Program Notes for Touching Light}

Touching Light is an original work for Percussionist and Mixed Reality Environment that explores the border areas between the physical world that we see around us, and the worlds of infinite possibility that each of us holds in our imagination.

This music is designed to be performed through a combination of physical instruments and virtual technologies through a medium called Mixed Reality. The Microsoft HoloLens, the device worn by the performer, allows the user to see and interact with holograms that exist invisibly in the space around the performer.

Like a Virtual Reality headset, the HoloLens allows the user to touch and interact with these holograms with their hands. By interacting with these holograms, the performer can press different types of 'buttons,' toggle 'switches,' and interact with all sorts of other objects. Unique to the HoloLens is the ability for those holograms to 'know' where physical objects are in the real world, and to respond appropriately; in Mixed Reality, you can bounce a virtual ball on the physical floor.

Touching Light features three different movements, each exploring a different sort of Mixed Reality interaction, asking the performer to control virtual objects and physical objects simultaneously. It is these simultaneous interactions that define 'music-making in Mixed Reality.'

The first movement, Simplicity, finds the performer acting as a sort of DJ, manipulating a virtual mixer to accompany themselves as they play a physical instrument. This movement asks the 
performer to move through a collection of different 'scenes' which require them to change 'the mix' so that different parts of the track get louder and quieter throughout the performance, until they are all present at the very end of the movement. This opening movement is inspired by EDM and hip-hop music and draws on modern jazz improvisation.

The second movement, Soliloquy, places the performer in the center of a sort of moving carousel made up of five different images chosen by the performer. As the five images rotate around them, the performer is tasked with responding to the images in a musical way, interpreting what they are seeing and feeling to the audience by translating those emotions into music. The movements is completed when the predetermined length of time has elapsed, usually coinciding with the first image returning to its original location. This second movement is inspired by ambient and avante garde music and draws on minimalistic and impressionistic styles.

The final movement, Synecdoche, provides the performer with a trio of cubes coated with an imaginary substance to make them very bouncy. In addition to that, the cubes also know how to play music! The performer is asked to move the cubes around the room, bouncing them off different surfaces, and then responding to the sounds the cubes make as they fly about the room. If you see a cube coming toward you, don't worry, it's not real...! This movement is inspired by non-western musical tonalities as well as the sort of whimsical, magical interactions that are naturally part of working in Mixed Reality.

The work was designed during the global coronavirus pandemic of 2020-21 and it is my hope that Touching Light reminds each of us that, despite everything, we are never truly alone; there is a world beyond this one if we are only willing to reach out and touch it. 
Appendix B: Touching Light (Traditional Score)

\section{Ian Riley}

\section{Touching Light}

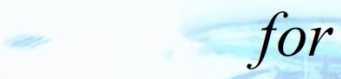

percussionist

$$
\text { and }
$$

mixed reality environment 


\section{Ian Riley \\ Simplicity \\ from Touching Light}


The first of three movements from the Touching Light suite, Simplicity is in many ways true to its name, while at the same time, like so many 'simple' things, deceptively complex. The movement asks the performer to move through a series of 'scenes,' as determined by the status of a virtual mixing board rendered in a holographic volume around the performer using the Microsoft HoloLens 2 and the companion application designed for this purpose.

The score indicates the content of each of the ten discrete channels present on the virtual mixer, and serve as a reference for the general length, shape, and timbre of each of the individual tracks. The instructions on the final page of the score designate the process by which the performer should interact with the virtual mixer, indicating the 'checkpoints' for various scenes.

In addition to manipulating the mixer, the performer is then asked to freely improvise, being inspired by the audio that is being generated by the application. The instrumentation for this movement is left to the discretion of the performer; historically successful iterations have included those on drumset, vibraphone, and found metals.

Touching Light for percussionist and mixed reality environment is an original suite of compositions to be performed in conjunction with the Microsoft HoloLens 2 companion application, also designed by the composer. Each movement in the suite explores a different combination of interactions between the performer, physical instruments, and virtual objects. Touching Light is the result of research culminating in a dissertation titled, 'Touching Light: $A$ Framework for the Facilitation of Music-making in Mixed Reality' by lan Riley from May 2021.

If you have any questions about this or any other movement, please reach out to me via email at ianthomasriley@gmail.com, or through my website, ianrileypercussion.com

\section{Equipment required:}

- Microsoft HoloLens 2 (or comparable XR platform)

- A method of streaming audio (and ideally video) from the device (the original performance used the Windows 10 HoloLens companion app to capture a live broadcast from the device, then sent audio and video to external speakers and projectors from the laptop running the companion app.)

- 2 stereo speakers (through which you will send the audio from the XR device)

- Audio cables, interface, etc. (dependent on your method of presenting audio) 


\section{Simplicity}

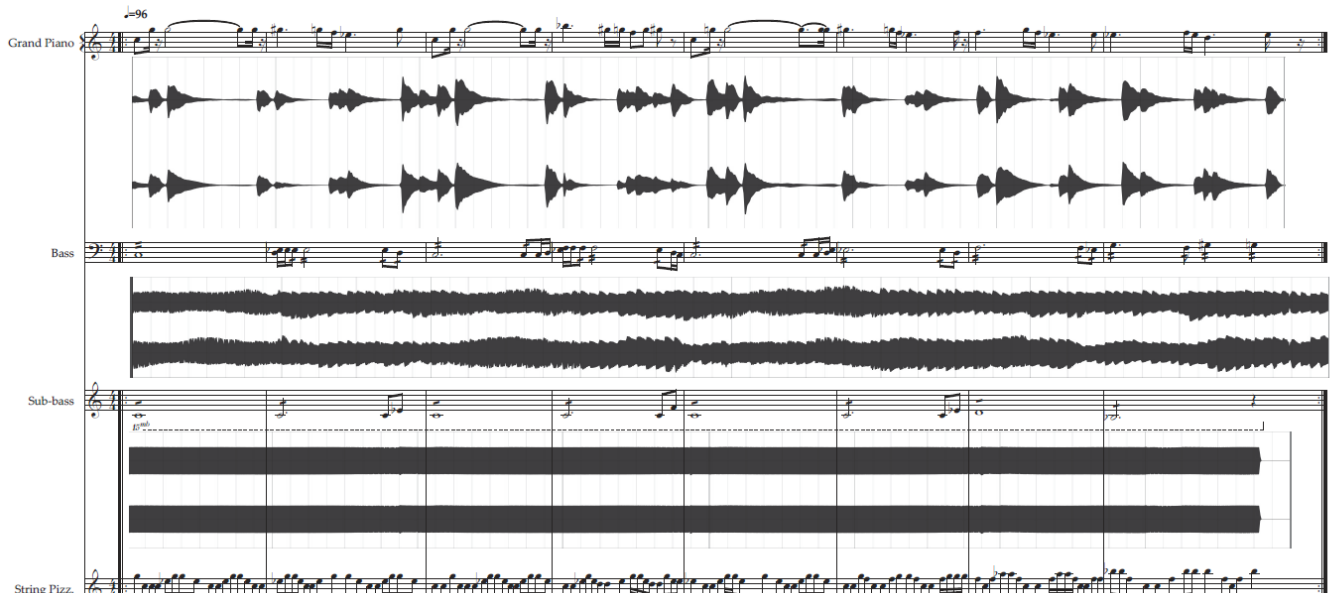

${ }_{\text {String Piz }}$

(1) 1

Drumstop

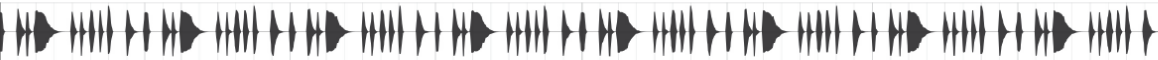

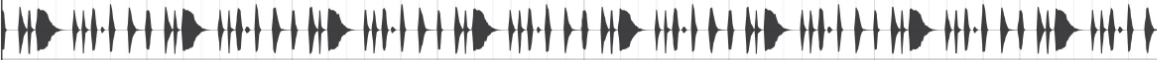

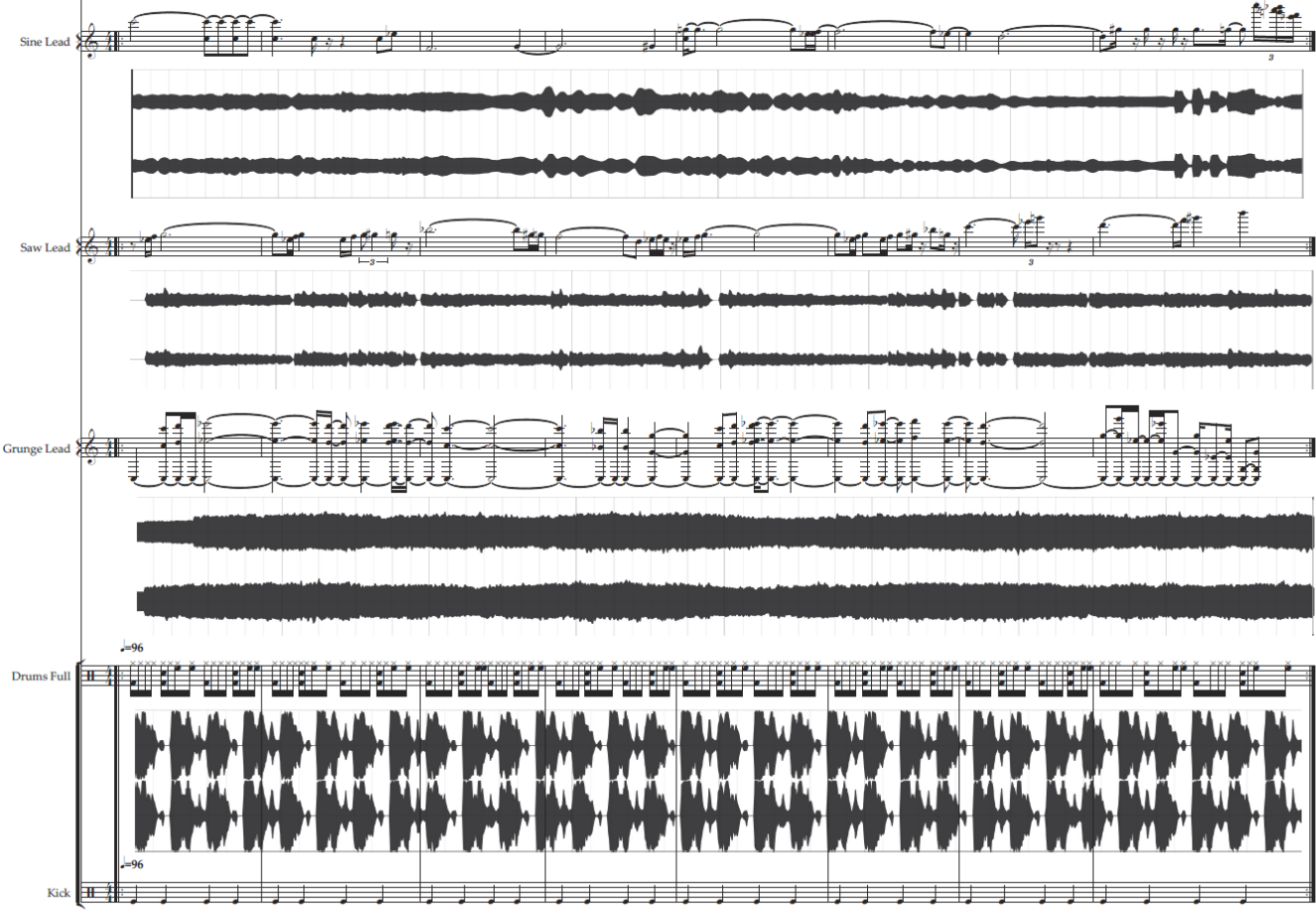

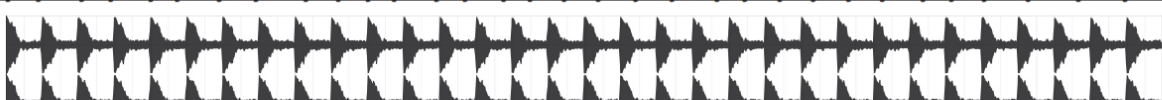

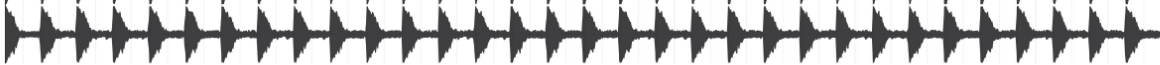




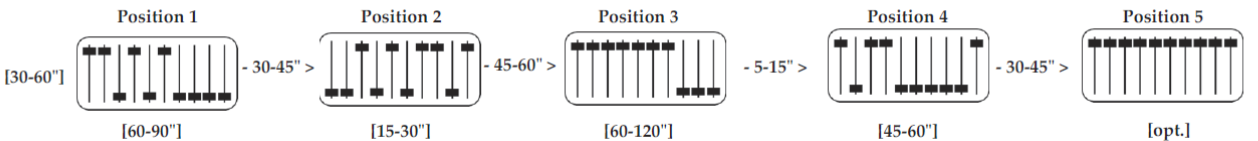

[15-30"]

[60-120"]

$[45-60 "$

[opt.]

After instantiating the application for the first movement, position the mixer in a location that is appropriate for ease of interaction without necessitating movement away from the instrument. Let us call this 'Position 0,' wherein no sound is being produced by the performer, the instrument, or the 'platform' (the XR device).

Position 0.5 (transitional)

For approximately 30-60 seconds ("comfortable"), improvise freely on the instrument in the key of C-minor (if using a melodic instrument). Consider exploring some of rhythmic and/or melodic motives described on the previous page, as these will appear when the pre-recorded audio begins to be mixed into the performance.

Once you have improvised for a sufficient amount of time, move toward Position 1 over the course of approximately 30-45 seconds (moderately).

Position 1

Using the virtual mixer, manipulate faders 1,2, 4, and 6 (approx.) until each is positioned at its maximum volume. Let this combination of piano, strings, bass, and synthesizer inspire your subsequent improvisation.

Position 1.5 (transitional)

For approximately 60-90 seconds ("relaxed"), improvise freely. The texture developed in this position will inspire future improvisation, so endeavour to not make it too complex at this juncture. Once your free improvisation has reached its conclusion, move toward Position 2 over the course of approximately 30-45 seconds (moderately).

Position 2

Using the virtual mixer, manipulate the faders until faders 3, 5, 7, 8 and 10 (approx.) are positioned at their maximum volume.

Let this combination of sub-bass, synthesizer, and drums inspire your subsequent improvisation.

Position 2.5 (transitional)

For approximately 15-30 seconds ("energized"), improvise freely, focusing on harmonic and/or timbral content rather than melodic or rhythmic content. Near the end of your improvisation, create a short, repeating pattern that can be performed with one hand, and continue to perform this pattern as you are able while you move toward Position 3 over the next 45-60 seconds (slowly).

Position 3

Using the virtual mixer, manipulate the faders until faders 3, 5, 7, 8 and 10 (approx.) are positioned at their maximum volume. Let this combination of piano, strings, bass, sub-bass, synthesizer, and drums inspire your subsequent improvisation.

Position 3.5 (transitional)

For approximately 60-120 seconds ("languid"), improvise freely, endeavoring to quote the harmonic, melodic, and rhythmic material presented by the mixer; use these quotations to focus the audience's attention on a specific element of the dense texture at a time. Once you have focused on at least three distinct elements, conclude your improvisation freely, moving toward Position 4 over the course of 5-15 seconds (quickly).

Position 4

Using the virtual mixer, manipulate the faders until faders 1, 3, 4 and 10 (approx.) are positioned at their maximum volume. Let this combination of piano, strings, sub-bass, and kick inspire your subsequent improvisation.

Position 4.5 (transitional)

For approximately 45-60 seconds ("conclusive"), improvise freely, recalling the material that you have established at Position 0.5 . Expand and elaborate on this material, drawing inspiration from the expansion and elaboration of the mixer's audio. Continue to complicate and vary your improvisation until you have reached a satisfying conclusion, understanding that the cycling audio will continue after the end of your improvisation.

Move to Position 5 over the next 30-45 seconds (moderately).

Position 5

Using the virtual mixer, manipulate the faders until all faders are positioned at their maximum volume. Let this combination of repeat for at least 1 complete cycle, and in this time (do no decide beforehand), decide if you will take a final improvisational solo over the full track, or if you will allow the audio to be the finale. In either case, also decide how you will stop the audio (i.e., suddenly using the master fader, element by element using individual faders, etc.)

Once all audio has become inaudible, the movement has concluded. 


\section{Ian Riley \\ Soliloquy \\ from Touching Light}


so·lil·o·quy (/sa'lilakwē/) noun. an act of speaking one's thoughts aloud when by oneself or regardless of any hearers.

The second movement of three movements from the Touching Light suite, Soliloquy asks the performer to freely improvise as they are inspired by a rotating carousel of landscapes from various parts of the world. In the original version, these five landscapes were representational images of a lush evergreen forest, a sunset over the ocean, a snow-capped mountain peak, a far-distant nebula, and foaming surf.

The instrumentation and musical content are left up to the performer; the composition included below is simply a representative performance of Soliloquy and may be performed as written, used for inspiration, or wholly ignored in favor of original improvisational material at the performer's discretion. The intention behind this work is for the performer to respond in some way to the images within a virtual carousel around them, reciting a sort of 'soliloquy' as their own thoughts and memories about the associated memories inspire a unique musical performance.

Touching Light for percussionist and mixed reality environment is an original suite of compositions to be performed in conjunction with the Microsoft HoloLens 2 companion application, also designed by the composer. Each movement in the suite explores a different combination of interactions between the performer, physical instruments, and virtual objects. Touching Light is the result of research culminating in a dissertation titled, 'Touching Light: A Framework for the Facilitation of Music-making in Mixed Reality' by lan Riley from May 2021.

If you have any questions about this or any other movement, please reach out to me via email at ianthomasriley@gmail.com, or through my website, ianrileypercussion.com

\section{Equipment required:}

- Microsoft HoloLens 2 (or comparable XR platform)

- Ideally a method to stream live video from the device for the audience 

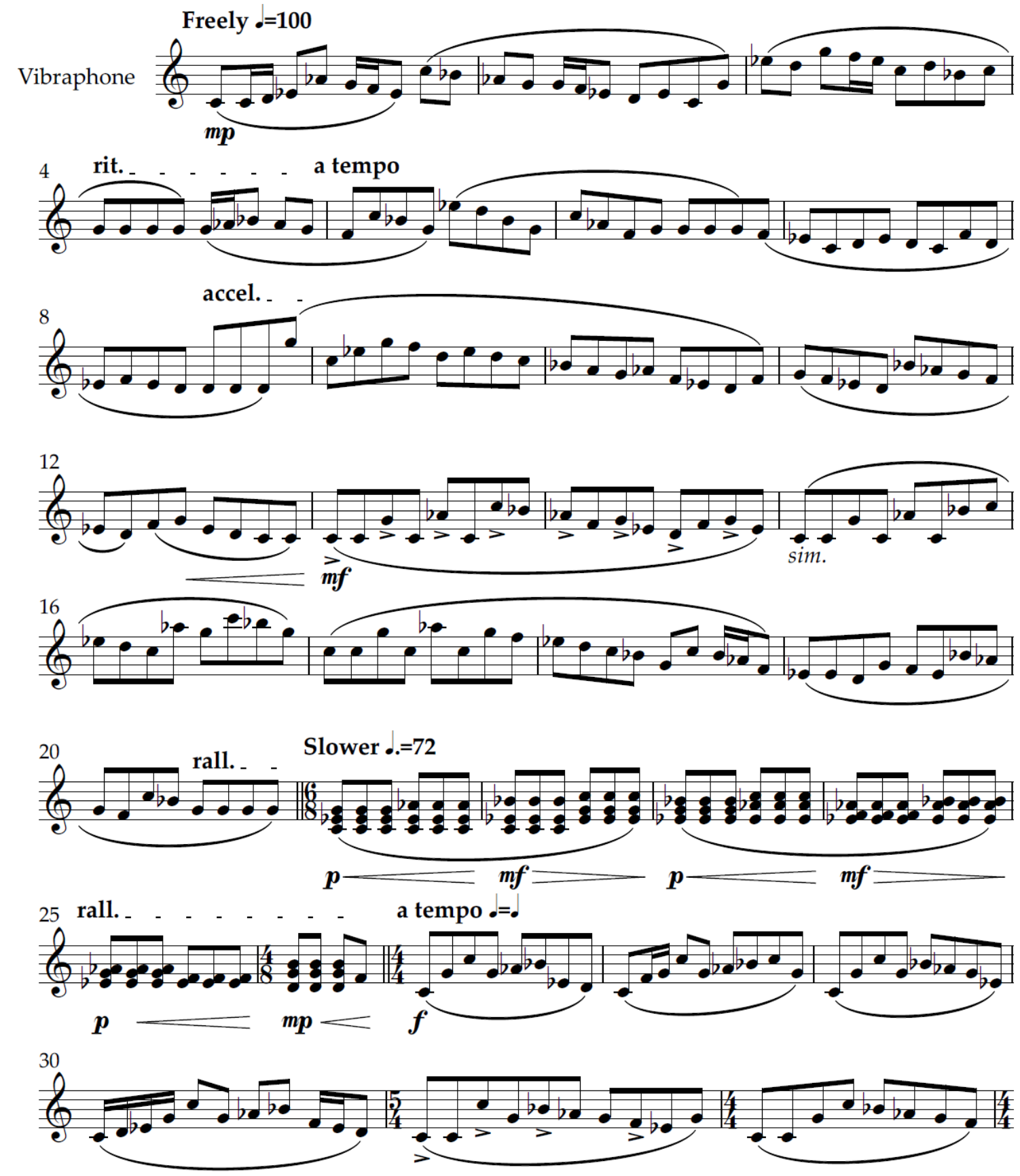
4

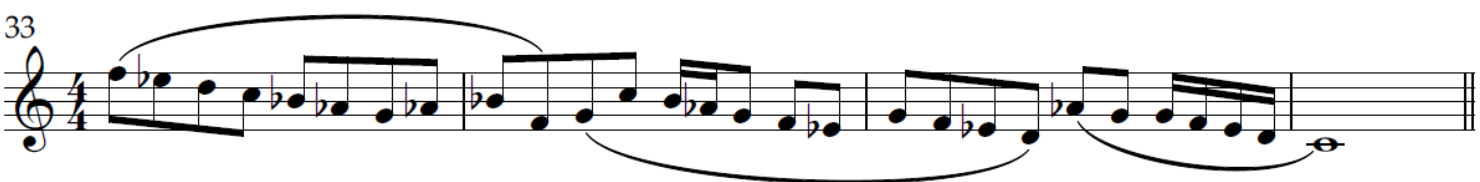

${ }_{37}$ Mysterious •=124

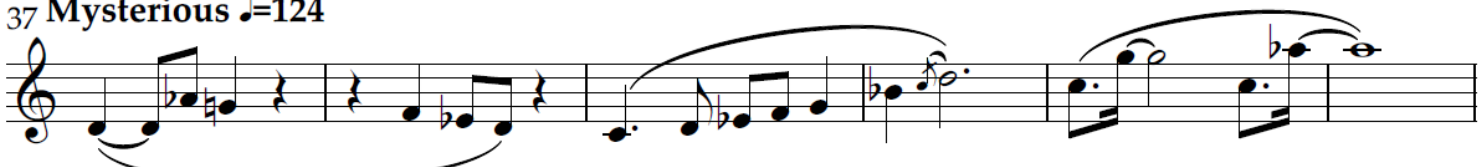
$m f$

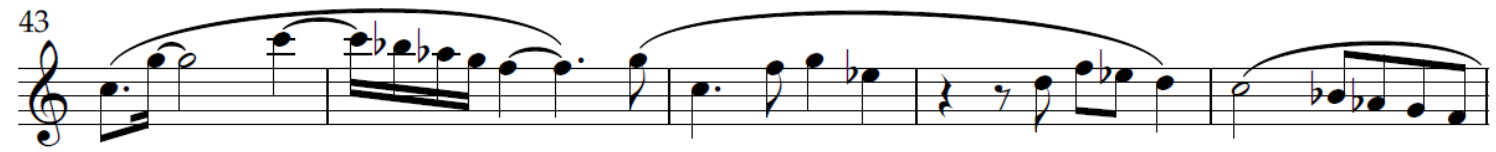

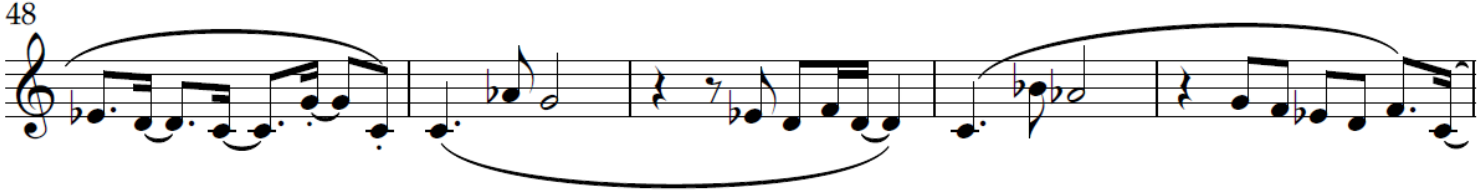

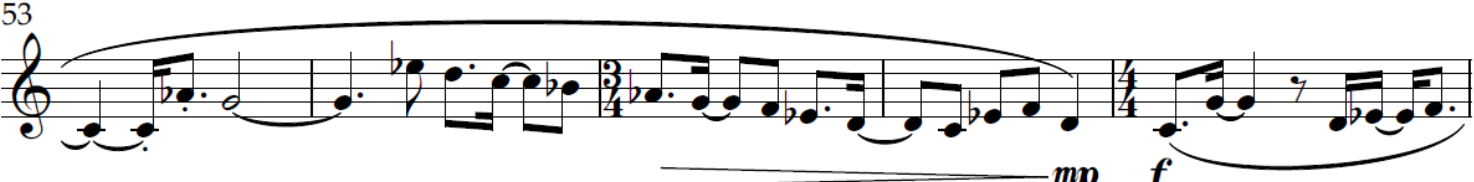

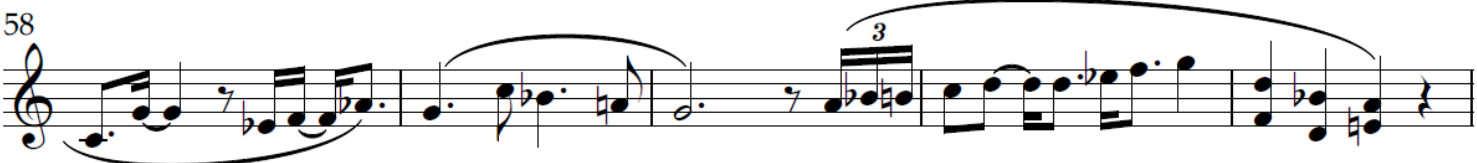
63 $\mathrm{C}_{0}^{68}$ (6) 
78
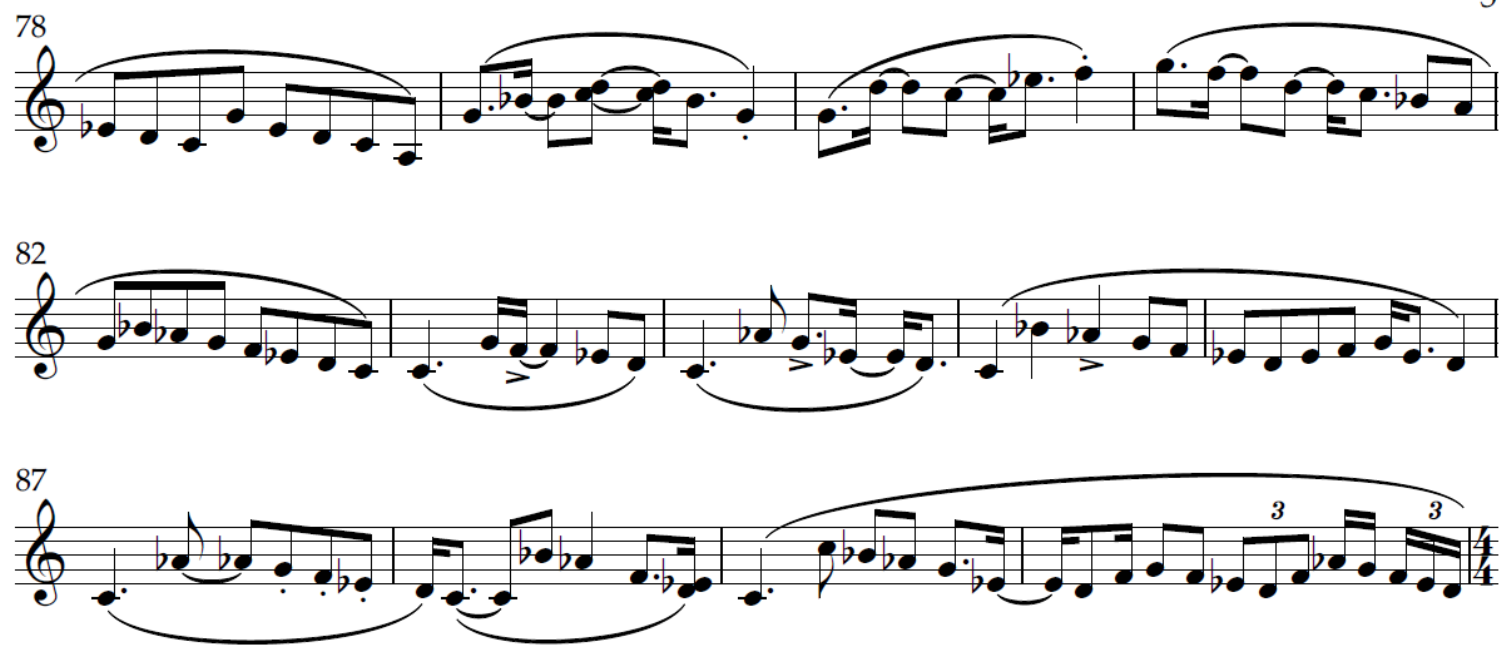

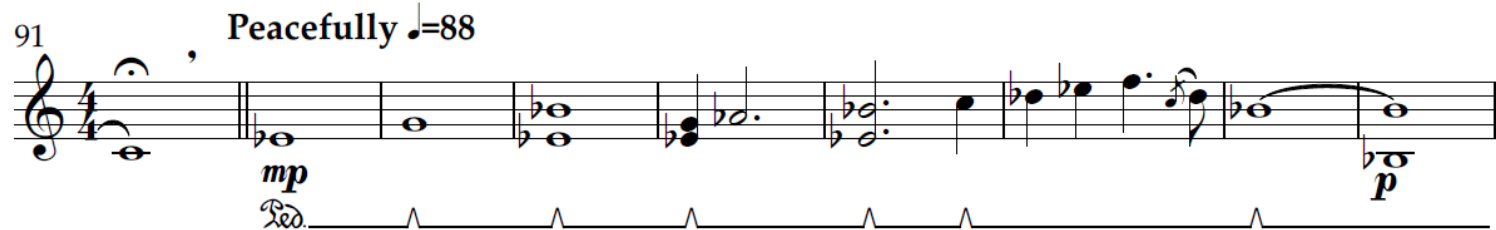

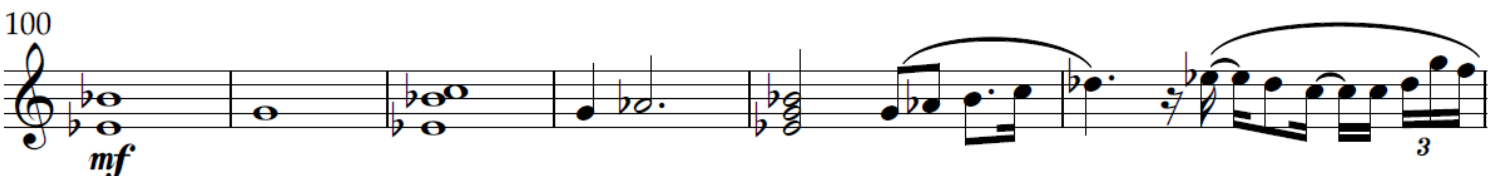

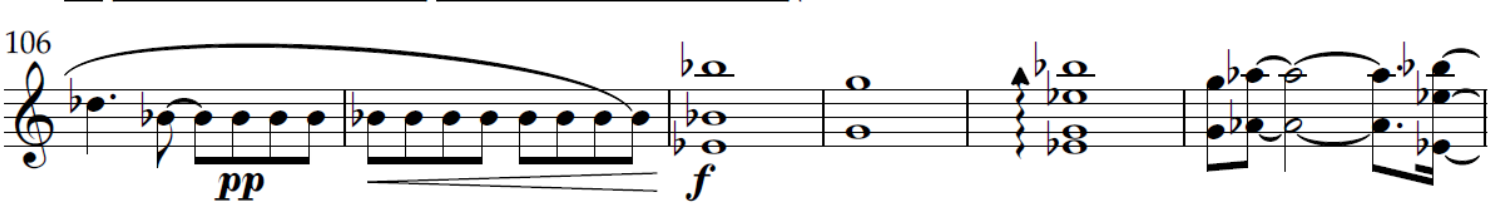
२อ. 1 गூ $\Lambda$

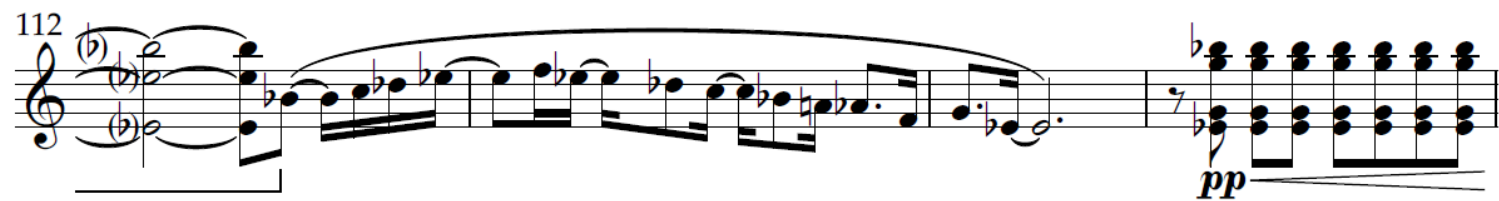

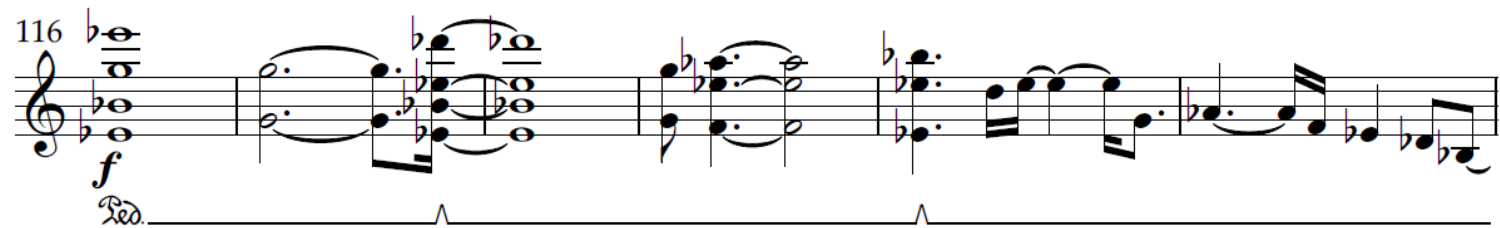

122 accel. _ - Moving forward $\bullet=108$

(6) 
6

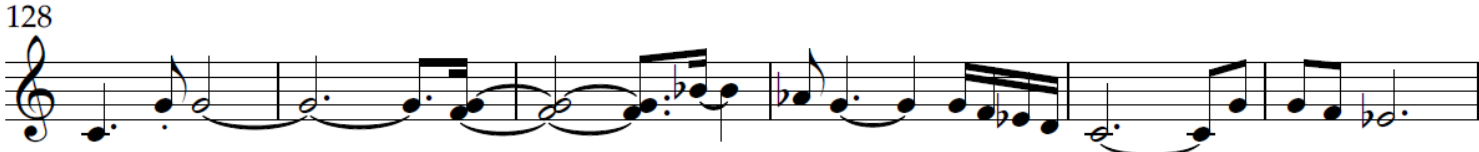

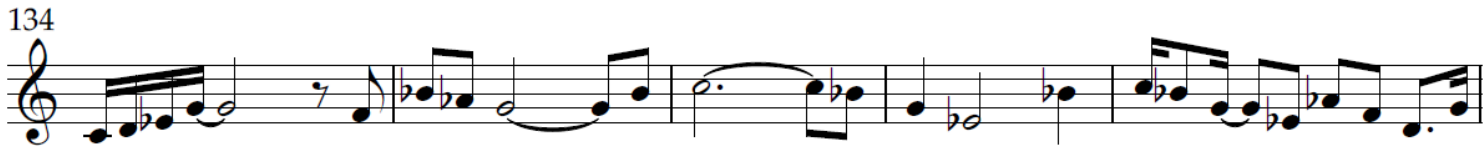
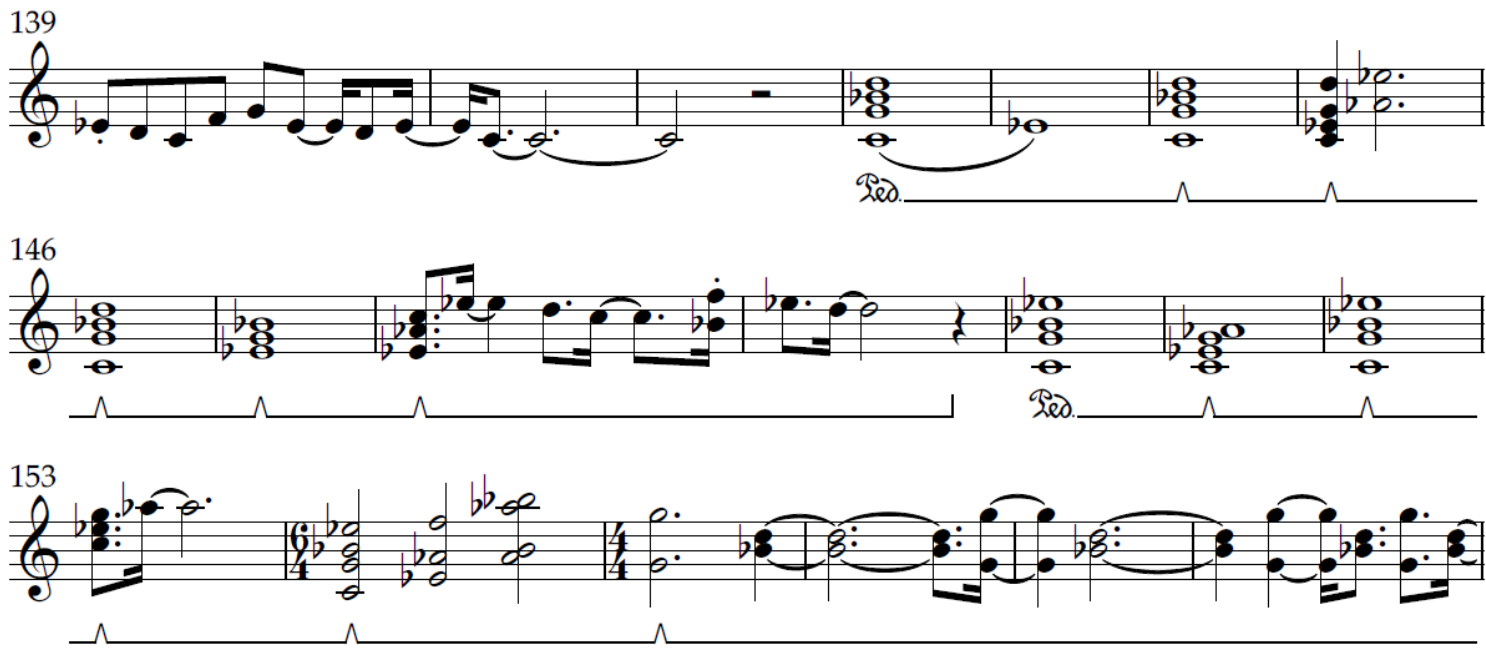

159 rit. _ _ - Undulating $\downarrow$ - $=96$

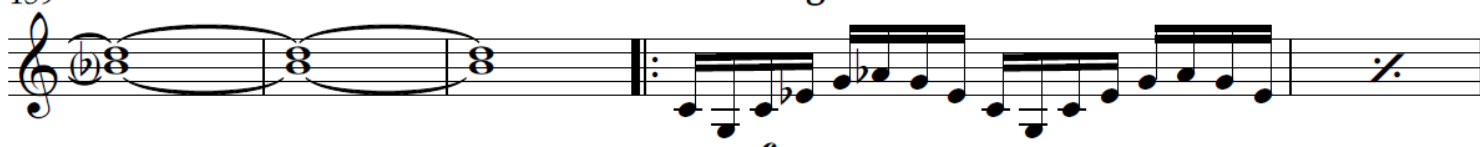
$m p-m f$

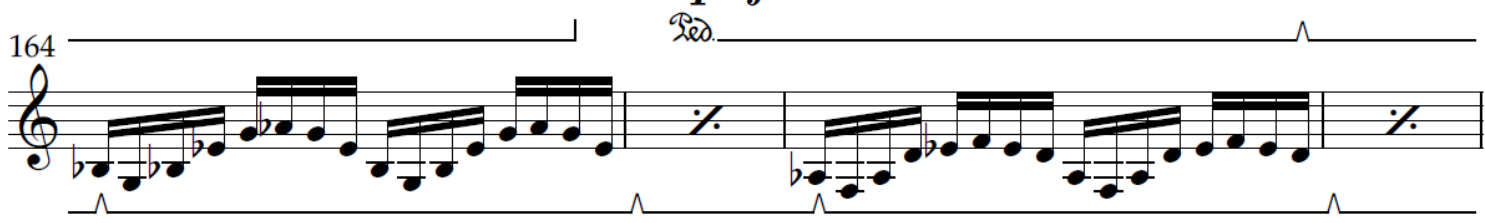

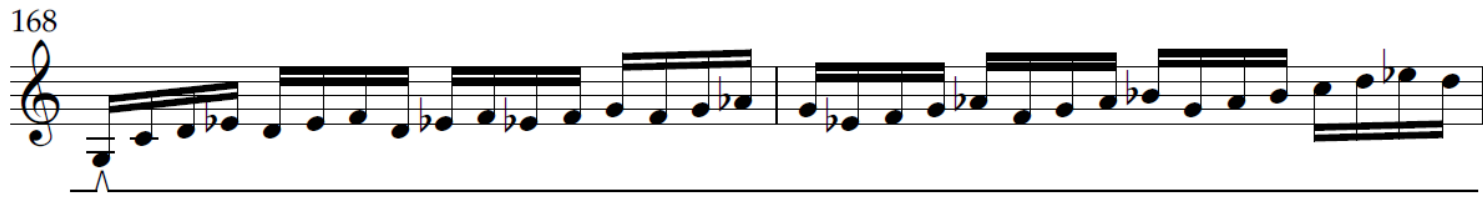

$\int_{10}^{170}$ 

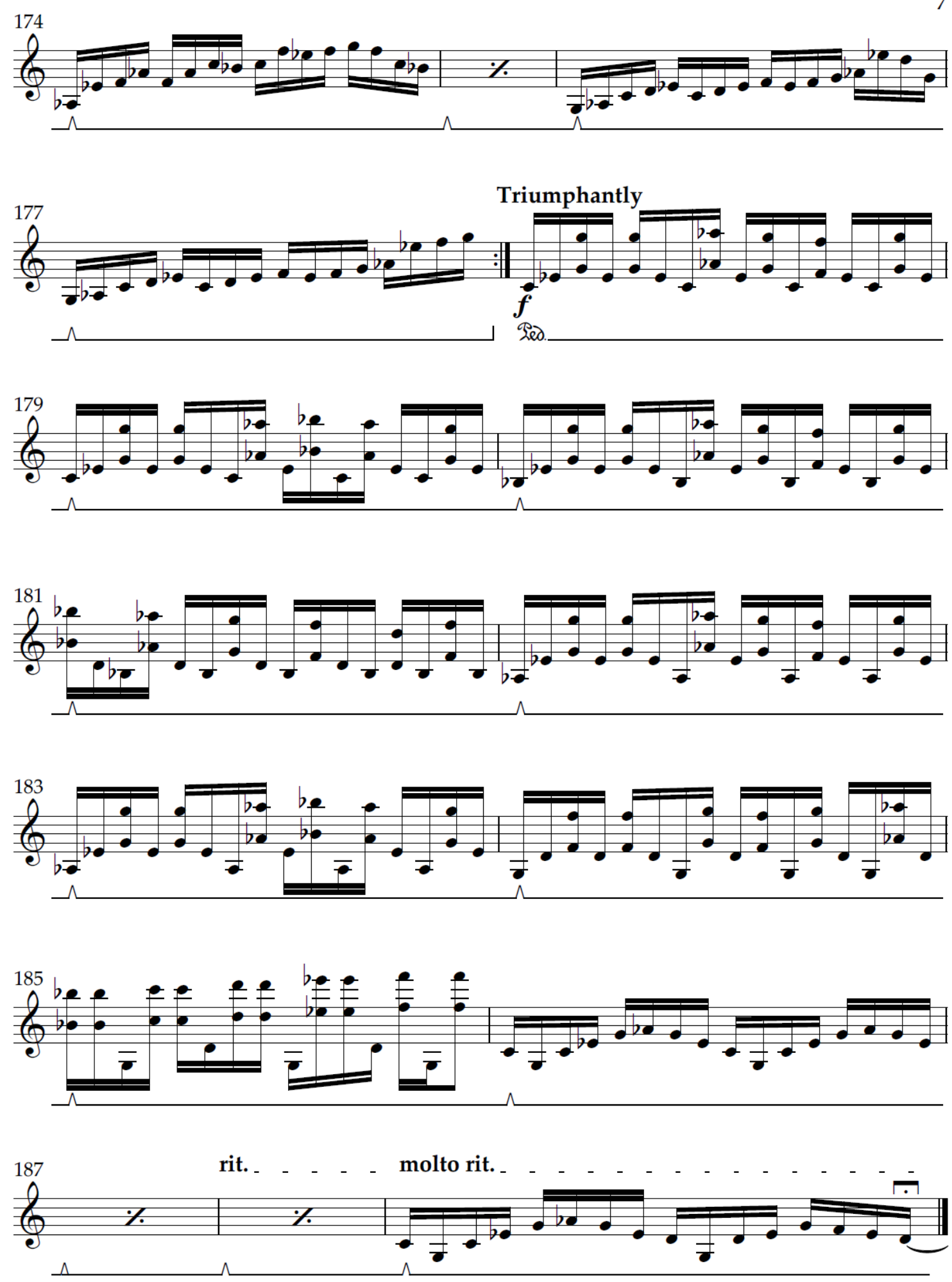

Page | 116 


\section{Ian Riley \\ Synechdoche from Touching Light}


syn'ec'do'che (/sa 'nekdake/) noun. a figure of speech in which a part is made to represent the whole, or vice versa.

The third of three movements from the Touching Light suite, Synecdoche asks the performer to explore both representation and abstraction. Centered around musical interactions in Mixed Reality, this movement draws on essentialized elements to explore the ways that we think about musicmaking.

The performer is presented first with a blank canvas; unlike the first two movements, no holographic objects will enter the volume without the explicit instruction of the performer. To begin, the performer will raise their left hand, palm facing them, to access a contextual 'hand-menu.' This interaction will facilitate many elements throughout the performance of this movement.

Upon selecting a particular icon on the menu, the performer will be presented with three virtual instruments within the holographic volume. Each of these three instruments have unique properties, both in comparison to physical instruments, as well as in comparison to one another.

These three instruments are visually represented by three apple-sized cubes of various color (red, green, and blue). Each cube has a specific number of different properties as programmed by the composer, but it is up to the performer to explore and discover as many of them as possible. The more properties that are identified, the more complete the representative nature of the cubes (and thus their synecdoche) becomes.

As in movements one and two, the physical instrumentation that will accompany this movement is left up to the performer. With this in mind, the notational score on the following pages is presented in a combination of timbral, melodic, and graphic notation. The performer may choose to interpret any or all of these 'scores' during the performance.

While the previous two movement of this suite focused heavily on the physical instruments involved, this final movement exists firmly in the shared space between the physical and virtual worlds, exemplifying this Mixed Reality.

Touching Light for percussionist and mixed reality environment is an original suite of compositions to be performed in conjunction with the Microsoft HoloLens 2 companion application, also designed by the composer. Each movement in the suite explores a different combination of interactions between the performer, physical instruments, and virtual objects. Touching Light is the result of research culminating in a dissertation titled, 'Touching Light: A Framework for the Facilitation of Music-making in Mixed Reality' by lan Riley from May 2021.

If you have any questions about this or any other movement, please reach out to me via email at ianthomasriley@gmail.com, or through my website, ianrileypercussion.com

\section{Equipment required:}

- Microsoft HoloLens 2 (or comparable XR platform)

- Ideally a method to stream live video from the device for the audience 
Synechdoche

from Touching Light

Ian Riley
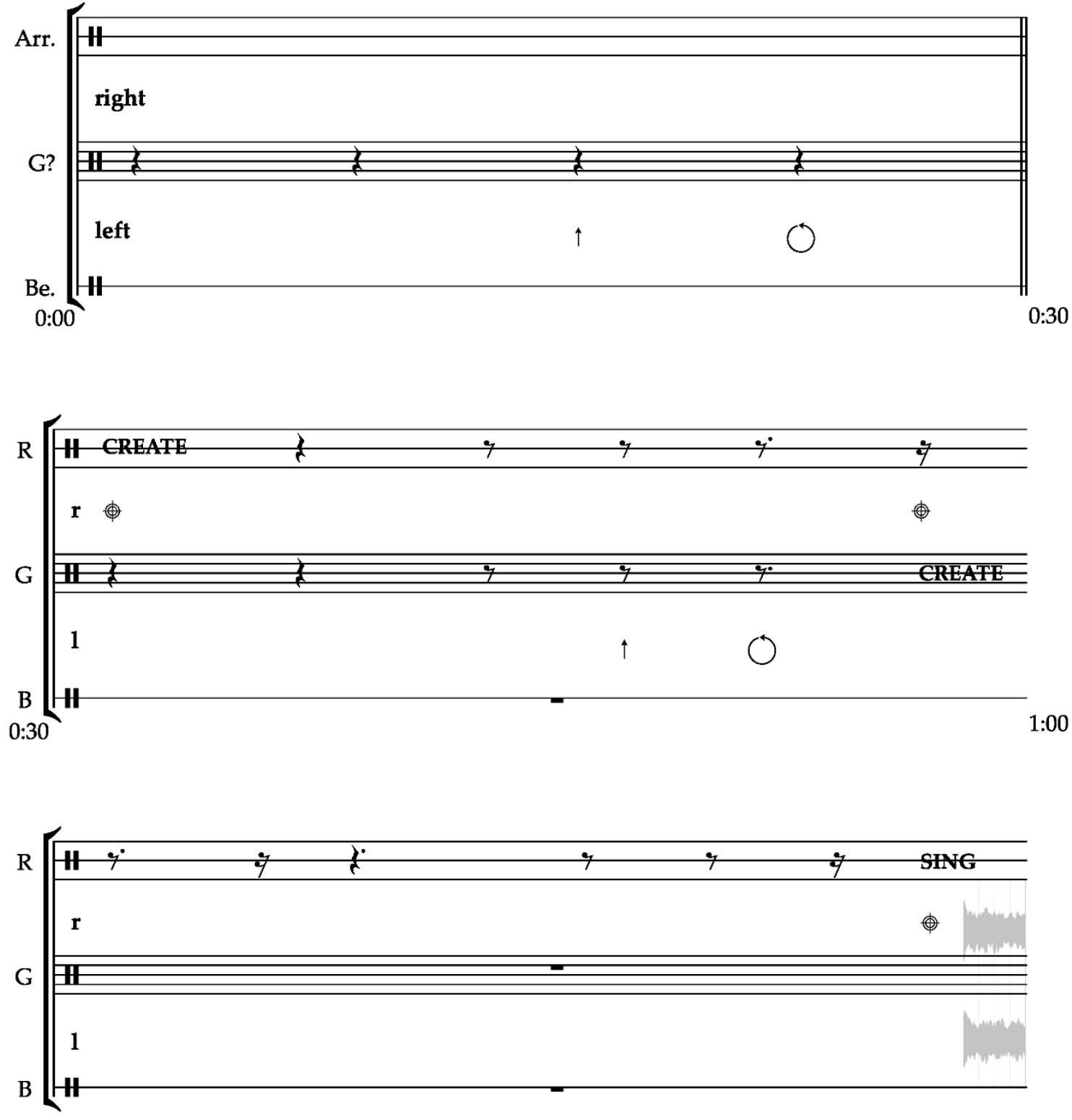

1:00

1:30

Page | 119 



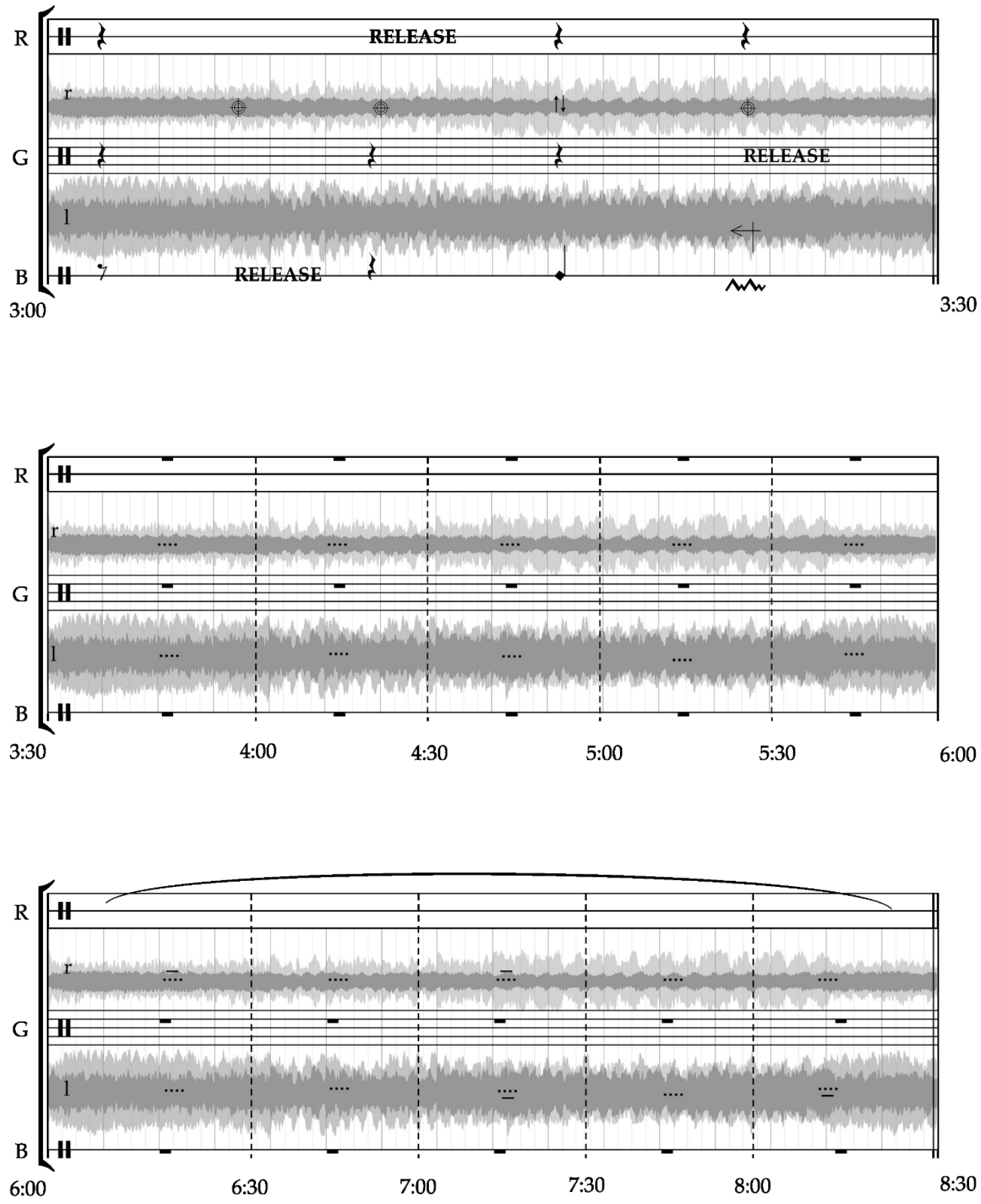
6

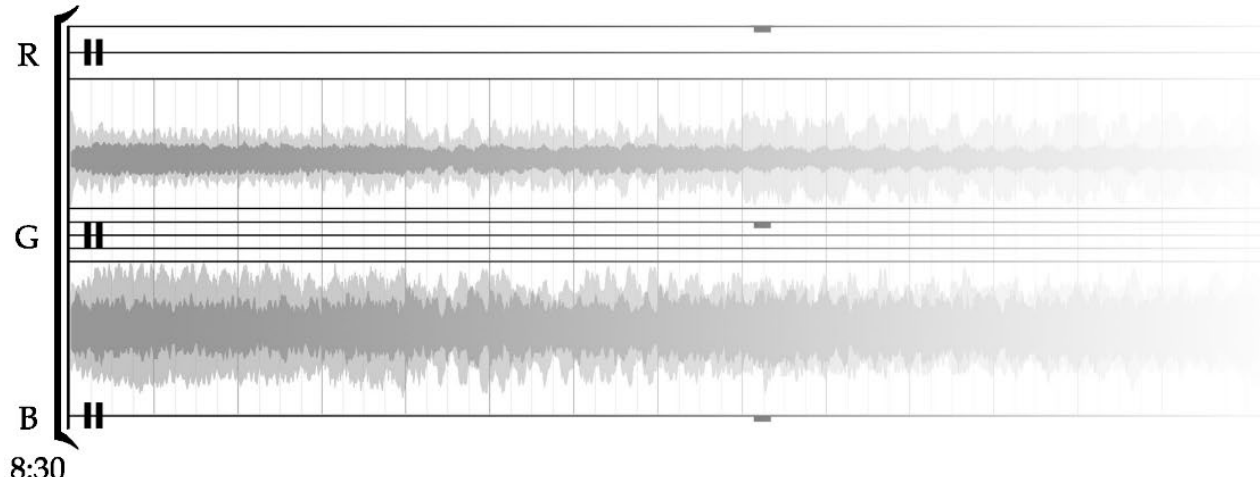

8:30
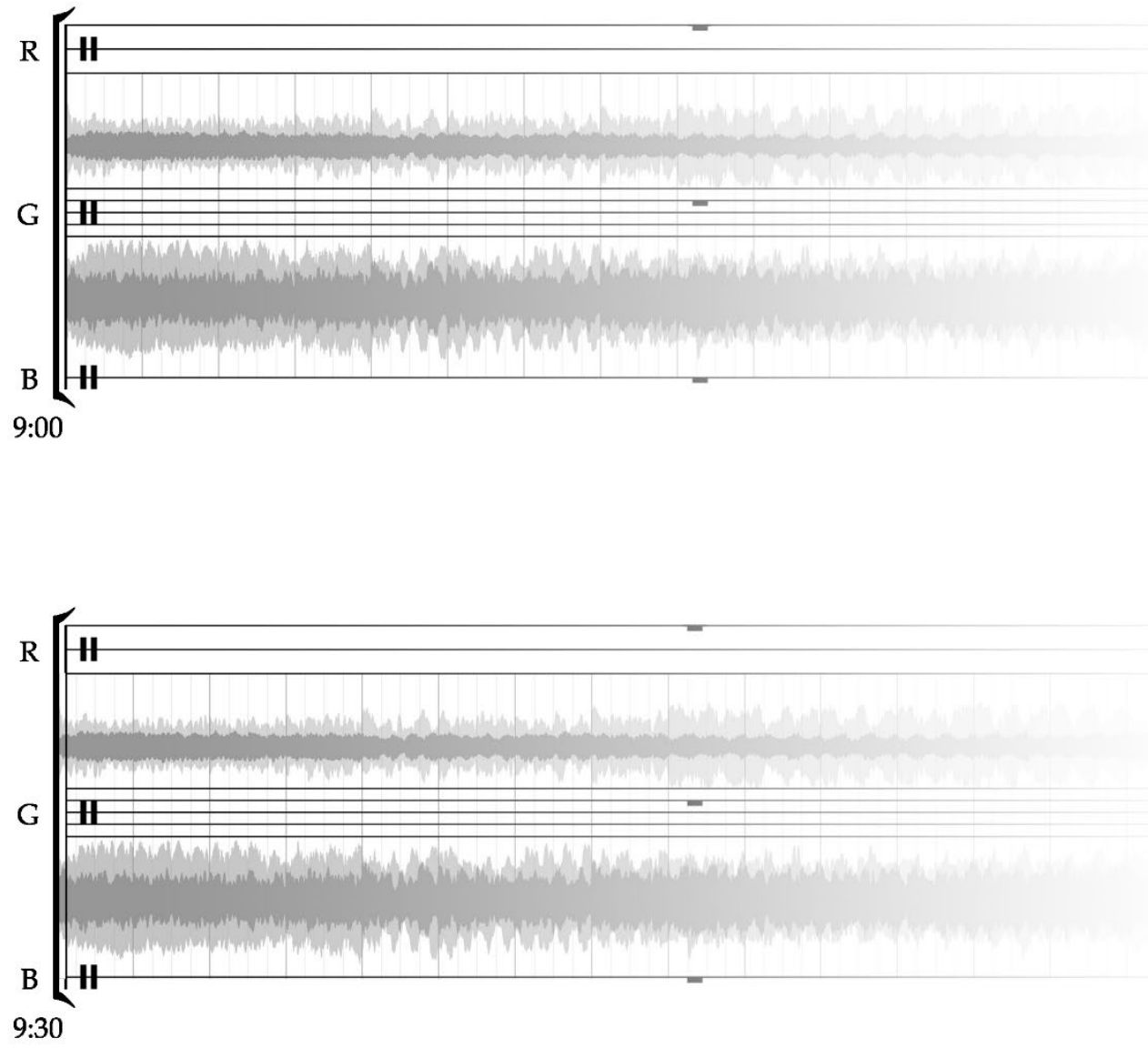

Page | 122 


\section{Appendix C: Touching Light (Unity Code)}

The full suite of files can be downloaded here:

https://drive.google.com/drive/folders/1LvQ2IzJgp461Gfi38g9SiUE4HbH_ktRE?usp=sharing

File Hierarchy Model

Touching Light (project) ${ }^{60}$

- Assets: this folder contains all sorts of components or 'assets' that ensure that the virtual objects and environments are rendered properly, ranging from different images and colors to apply to $3 \mathrm{D}$ objects to the audio samples that are present in the final environment.

O AudioSamples: contains all the audio samples in the project

○ Materials: contains all of the textures and 'shaders' in the project

O MRTK: contains the Microsoft MR Toolkit which allows compatibility with the HoloLens 2

○ Plugins: contains information for various other 'helper' programs

○ Scenes: contains information about specifically stages 'scenes,' and helps to determine where objects should appear when an application starts up

○ MixLevels.cs: a custom script that links the virtual mixer to the native Unity audio mixer

- Builds: contains various versions of different parts of the final experience

\footnotetext{
${ }^{60}$ Including all the file names (notwithstanding the C\# code for all of the tiles) within the project directly in this document would require hundreds of pages of largely meaningless text; instead, this model presents the general relationships between the macro elements of the project in an effort to define the shape of the score in a communicable manner. I have also included a select few $\mathrm{C \#}$ scripts that are short enough to be meaningful in this context and that serve as the crux of the core interactions for each movement.
}

Page $\mid 123$ 
○ Simplicity_mixer: the final version of the application for movement 1

○ Soliloquy_carousel: the final version of the application for movement 2

○ Synecdoche_cage: the final version of the application for movement 3

- Library: this folder contains all the various non-programming elements needed for the application to run as intended

- Logs: this folder contains various error and system logs, records of events that occurred when the program was running

- Packages: this folder contains a reference for the necessary packages required for the applications to run correctly.

- ProjectSettings: this folder contains reference lists for various settings that the application needs in order to run as intended. 


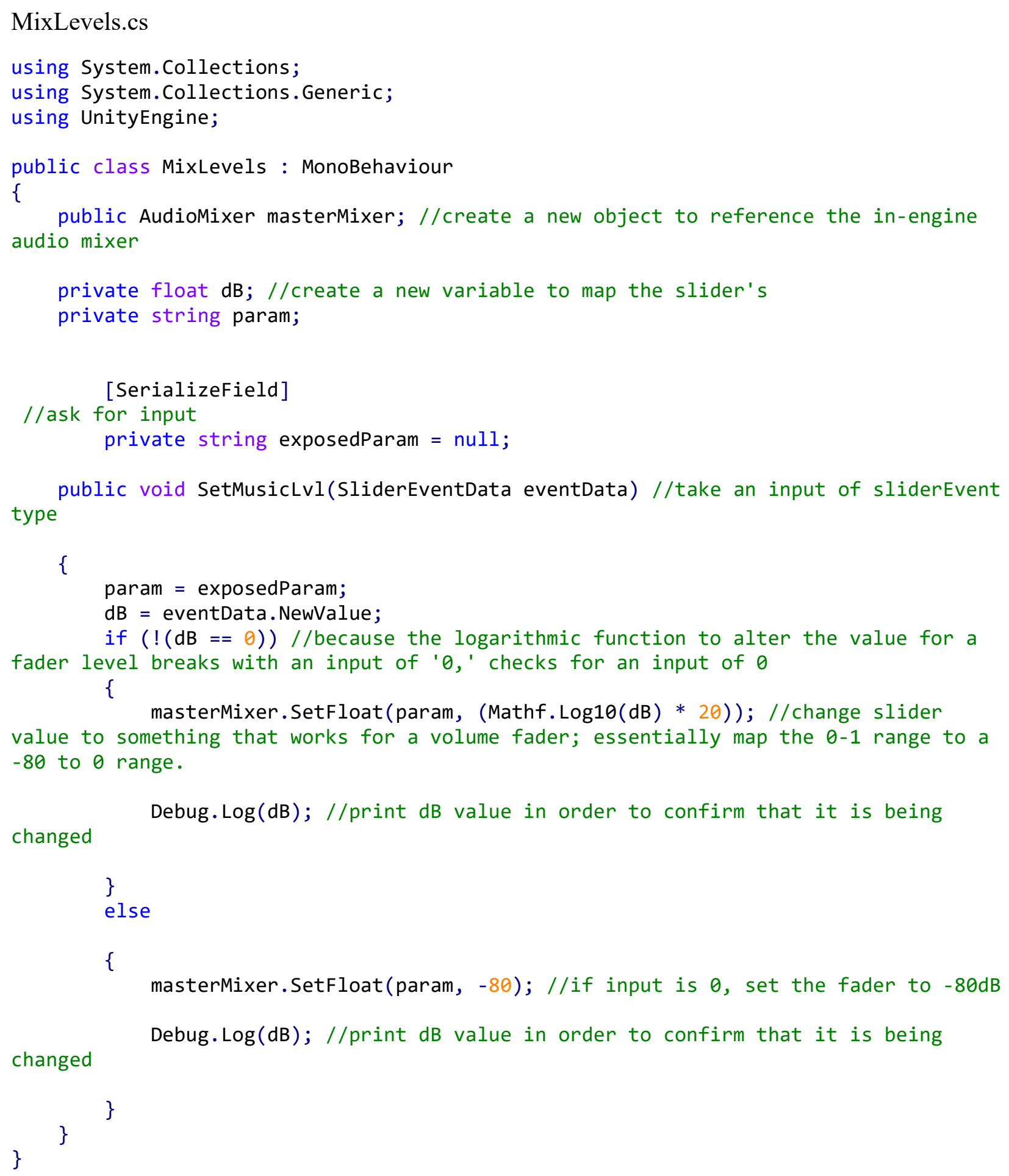




\section{SetToOrigin.cs}

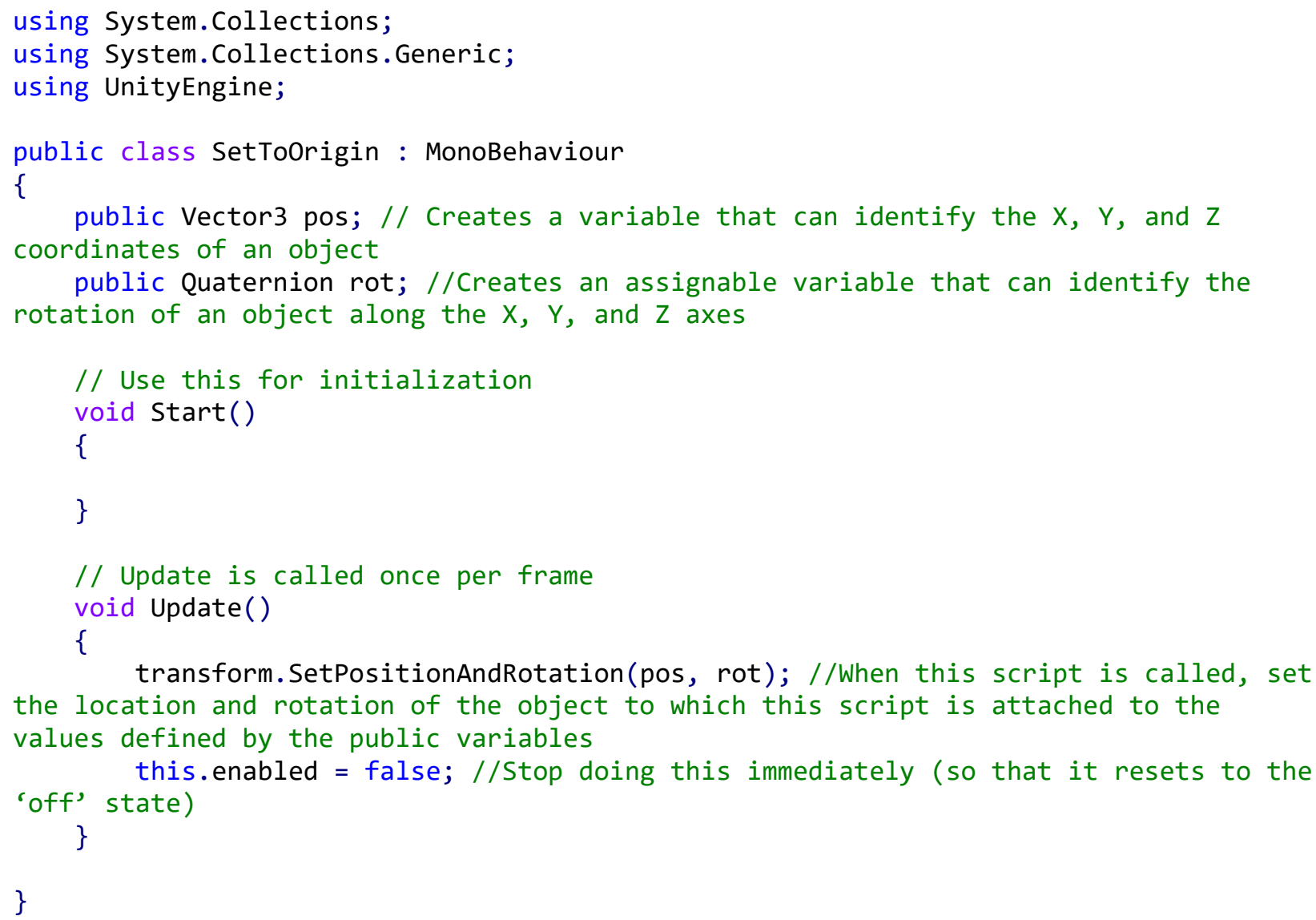




\section{ObjectPuller.cs}

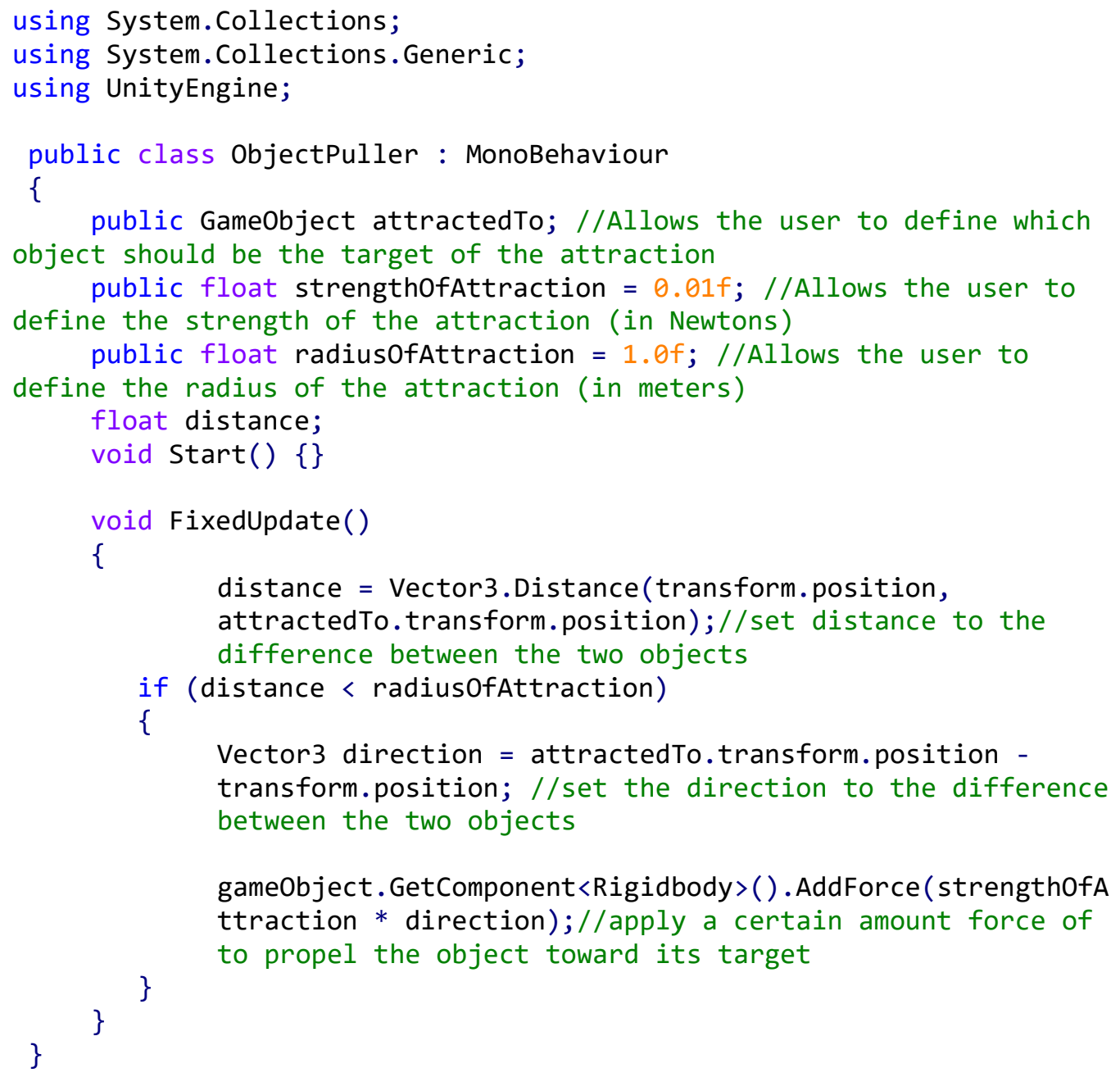




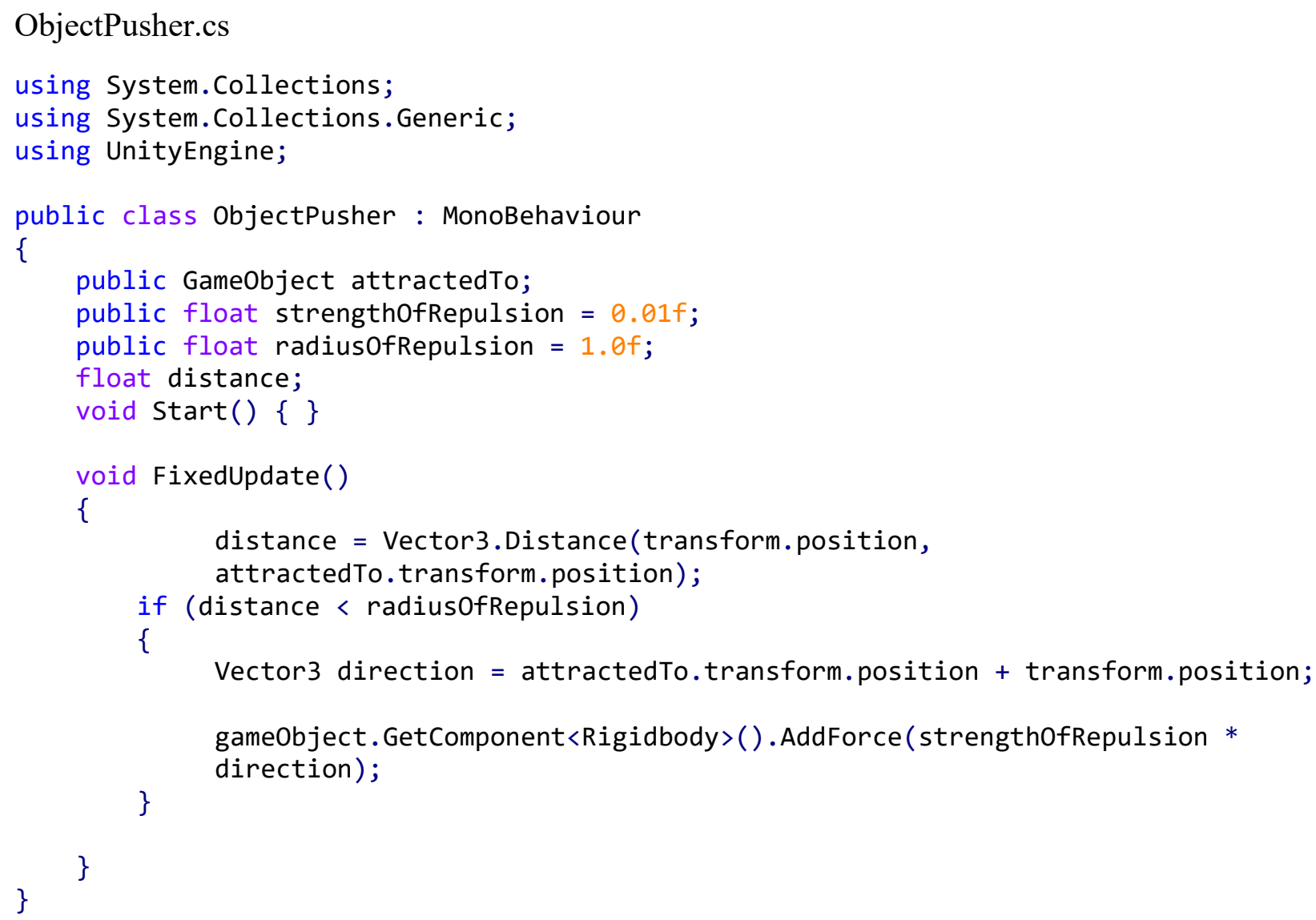




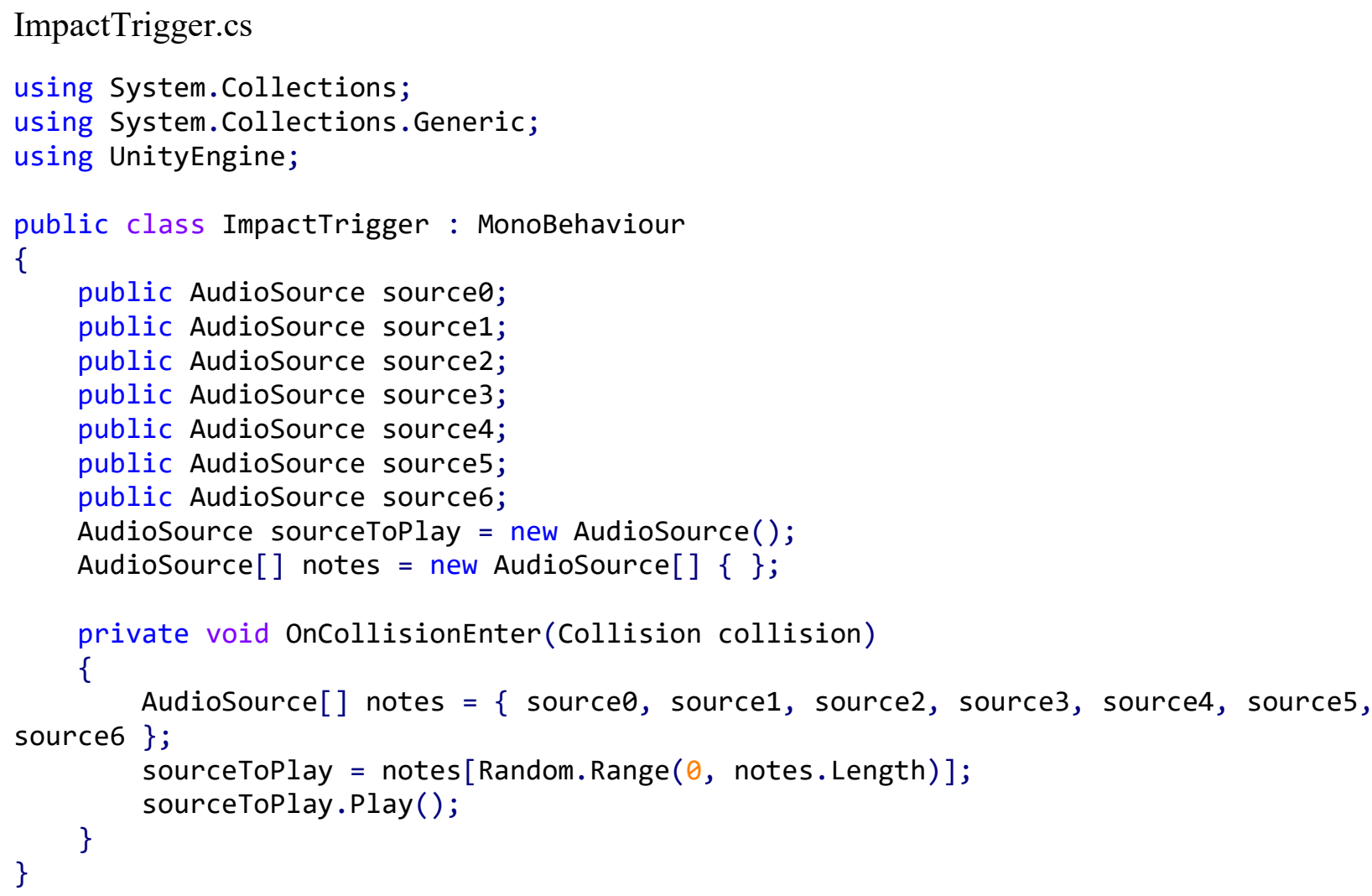




\section{Appendix D: Touching Light 'dev-log' Blog Posts}

As part of the development process for Touching Light, in order to most closely emulate the SCRUM prototyping methodology, I kept a public 'development log' accessible via my website which will remain in place indefinitely. For the purpose of preserving these articles, their full text, along with URLs to videos referenced in the posts have been recreated here in full, as posted, inclusive of typographical and grammatical errors.

renders $(03 / 18 / 21)$

Posted on $\underline{\text { March 18, } 2021}$

By ianthomasriley

\section{In $\underline{\text { Uncategorized }}$}

Exciting times! Lots of things going on at WVU, both related to my dissertation, and otherwise. With midterms having just concluded, we enter the final stages of planning for our virtual percussion ensemble concert, student juries, and subsequently my final recital... luckily, I seem to have some apps that work!

The past month has been a lot of keeping my nose to the grindstone and iterating on the development of the final movement of Touching Light, 'Synecdoche' (or 'the one with cubes on' as it has been called). I'll include a video at the end of this post that shows the app for the movement in action.

The artistic goal for this movement was to explore musical interactions that were unique to MR; while movements 1 and 2 engage MR to broaden the possibilities of live performance, ultimately both the holo-mixer and the carousel could be achieved via other means. The weightless projections and interactions of the holographic objects in movement 3 are a different story. Page $\mid 130$ 
There were three main things that I wanted to do with Synecdoche. The first one was to make 'primitives,' (in this case, cubes) somehow the 'star of the show.' I started my 3D modeling experience with Blender, and so the 'meme' about deleting the default cube may have directly inspired this in more ways than one. So, the first step was to figure out what I could do to make the cube(s) interesting.

As I've shown before, I colored the cubes so that they corresponded to the three principal colors in the RGB color profile, with some edge-lighting to make them a bit more abstract, and then made them weightless.

For the final version, I've spent a lot of time on the HandMenu, building out specific controls for each cube individually, as well as some global controls. 


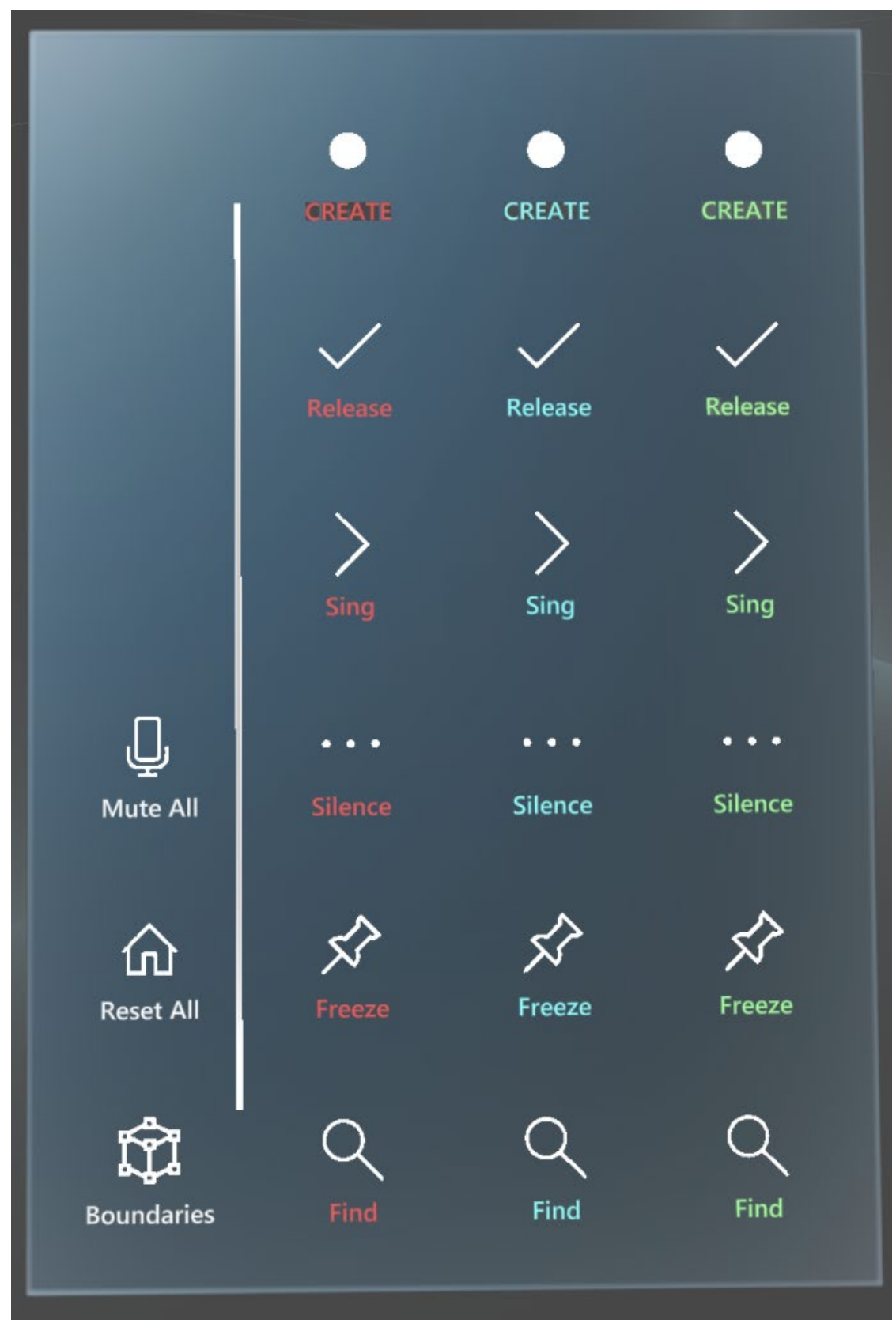

Mute All command will forcibly mute the Sound Sources on all of the Cubes, using the boolean operator in the Audio Source

Reset All will reset all of the cubes to the origin, without removing any inertia that they may be carrying using a custom script that called SetToOrigin 
1 using System.Collections;

$2 \quad$ using System.Collections.Generic;

3 using UnityEngine;

4

5 public class SetToOrigin : MonoBehaviour

6\{

$7 \quad$ public Vector3 pos;

8 public Quaternion rot;

9

$10 \quad / /$ Use this for initialization

$11 \quad$ void Start()

12\{

13

$14 \quad\}$

15

16 // Update is called once per frame

17 void Update()

18\{

Page | 133 
19 transform.SetPositionAndRotation(pos, rot);

$20 \quad$ this.enabled $=$ false;

$21 \quad\}$

22

$23\}$

Boundaries generates the cage around the performer which helps keep the cubes within a reasonable performance space

\section{CREATE}

The Create commands enables the Mesh Renderer on each of the cubes (which is disabled by default)

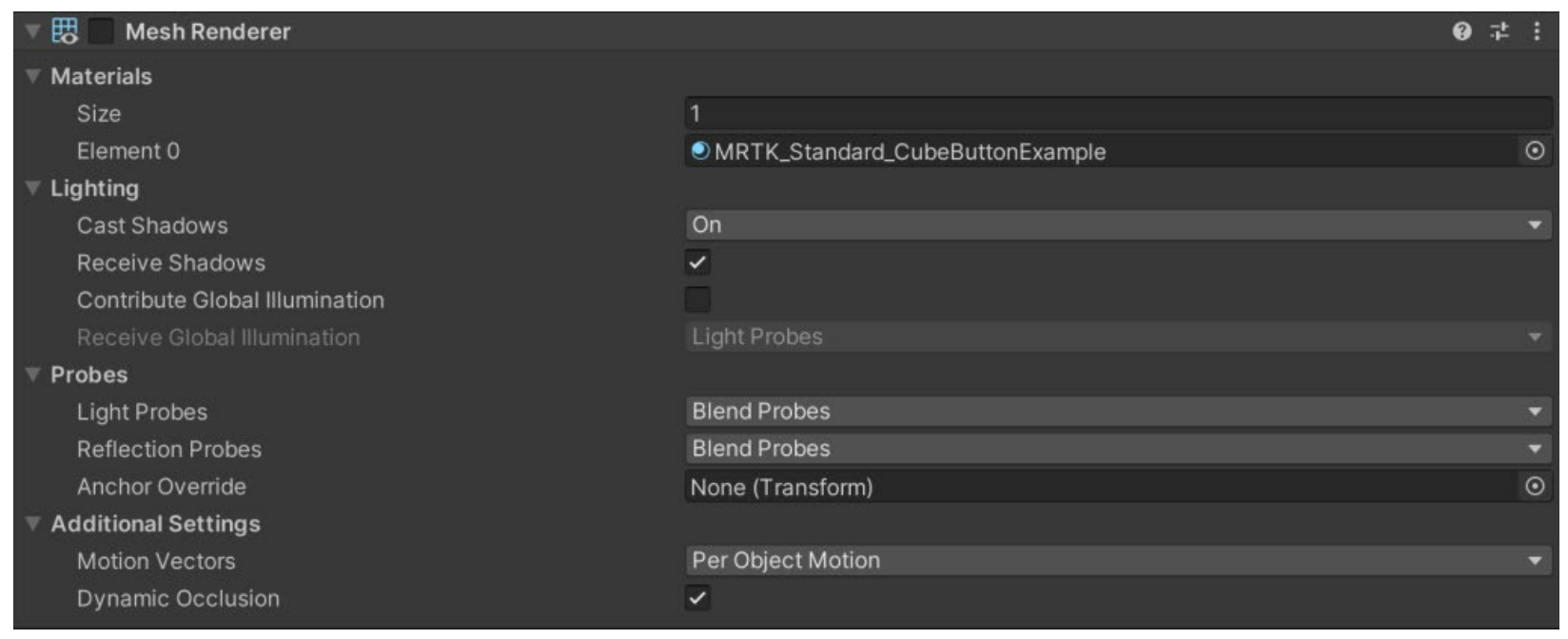

\section{RELEASE}

The Release commands activate a few different functions, unique to each cube.

\section{Release RED}

Page | 134 
When released, the Red Cube activates the 'Object Puller' script, which causes the Red Cube to be pulled toward a designated object (the Blue Cube), if it is within range using the ObjectPuller custom script:

$1 \quad$ using System.Collections;

$2 \quad$ using System.Collections.Generic;

3 using UnityEngine;

4

5 public class ObjectPuller : MonoBehaviour

6\{

7 public GameObject attractedTo;

8 public float strengthOfAttraction $=0.01 \mathrm{f}$;

9 public float radiusOfAttraction $=1.0 \mathrm{f}$;

10 float distance;

11 void Start() \{\}

12

13 void FixedUpdate()

14\{

15 distance $=$ Vector3.Distance(transform.position, attractedTo.transform.position); 
$16 \quad$ //Debug.Log(distance);

17 if (distance $<$ radiusOfAttraction $)$

18\{

19 Vector3 direction $=$ attractedTo.transform.position - transform.position;

20 gameObject.GetComponent $<$ Rigidbody $>$ ().AddForce(strengthOfAttraction *

21 direction);

$\left.\begin{array}{l}22 \\ 23\end{array}\right\}$

\}

\section{Release BLUE}

When released, the Blue Cube activates the 'Object Pusher' script, which causes the Blue Cube to be pushed away from a designated object (the Green Cube), if it is within range using the ObjectPusher custom script:

1 using System.Collections;

$2 \quad$ using System.Collections.Generic;

3 using UnityEngine; 
5 public class ObjectPusher : MonoBehaviour

6\{

7 public GameObject attractedTo;

$8 \quad$ public float strengthOfRepulsion $=0.01 \mathrm{f}$;

9 public float radiusOfRepulsion $=1.0 \mathrm{f}$;

10 float distance;

$11 \quad$ void $\operatorname{Start}()\{\}$

12

13 void FixedUpdate()

14\{

15 distance $=$ Vector3.Distance(transform.position, attractedTo.transform.position);

$16 \quad$ //Debug. $\log ($ distance $)$;

17 if $($ distance $<$ radiusOfRepulsion $)$

18\{

19 Vector3 direction $=$ attractedTo.transform.position + transform.position;

20 gameObject.GetComponent $<$ Rigidbody $>$ ().AddForce(strengthOfRepulsion *

21 direction); 


\section{Release GREEN}

When released, the Green Cube is affected by gravity, causing it to accelerate downwards at approximately the same rate as an object on earth's moon. No custom scripts are required for this interaction; instead the button toggles the 'Use Gravity' toggle on the cube's RigidBody.

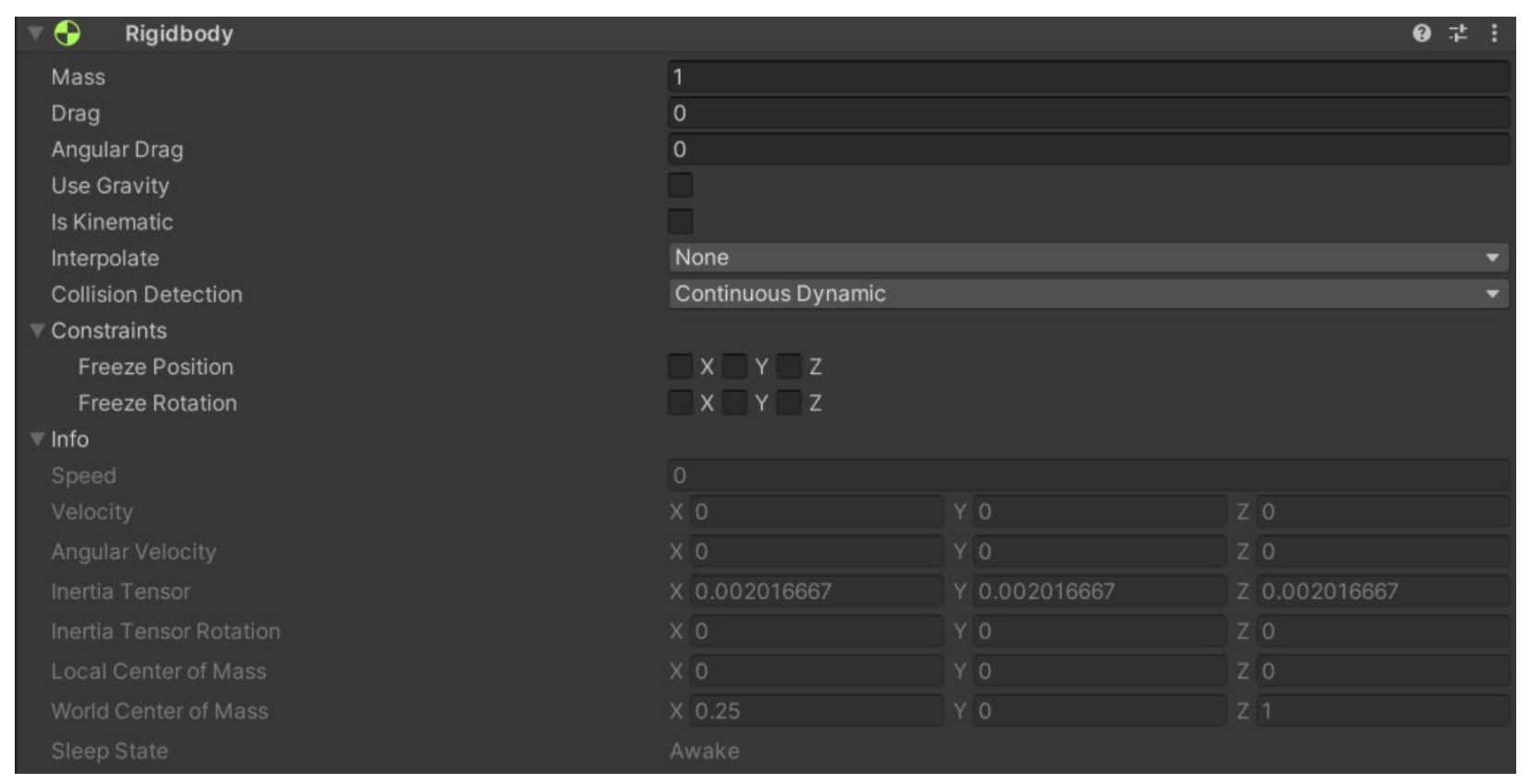

\section{SING}


Each of the cubes has a different function when 'Singing,' but RED and GREEN are functionally the same. Both cubes’ Audio Source is unmuted (it begins muted).

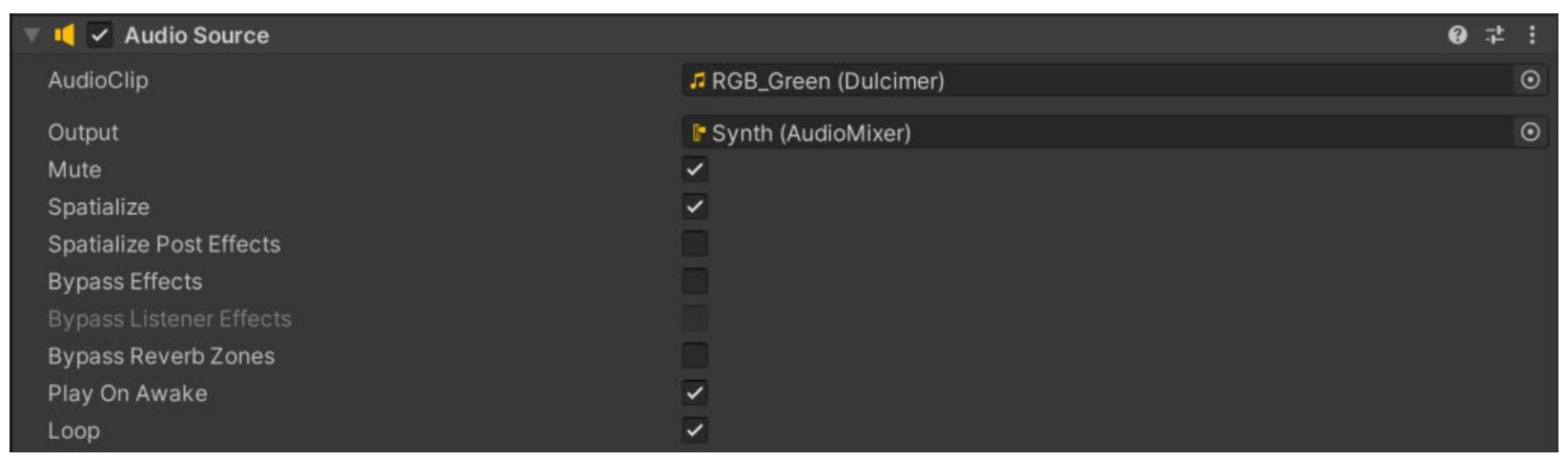

This allows the tracks that they play to remain in sync, as they play on load.

For the BLUE cube, the 'SING' button activates a custom script that plays a randomized diatonic note whenever the cube detects a collision, using the ImpactTrigger custom script:

1 using System.Collections;

2 using System.Collections.Generic;

3 using UnityEngine;

4

5 public class ImpactTrigger : MonoBehaviour

6\{

7 public AudioSource source0;

8 public AudioSource source1;

9 public AudioSource source2; 
10 public AudioSource source3;

11 public AudioSource source4;

12 public AudioSource source5;

13 public AudioSource source6;

14 AudioSource sourceToPlay = new AudioSource();

15 AudioSource[] notes = new AudioSource[] \{\} ;

16

17 private void OnCollisionEnter(Collision collision)

18\{

19 AudioSource[] notes $=\{$ source0, source1, source2, source3, source4, source5, 20 source6 \};

21 //Debug.Log(notes[Random.Range(0, notes.Length)]);

22

sourceToPlay $=$ notes $[$ Random $\cdot$ Range $(0$, notes.Length $)]$;

23

sourceToPlay.Play();

$24 \quad 3$

\} 
Those sources are defined through the Impact Triger (Script) component, and are attached as separate audio sources and children of Blue_Cube.

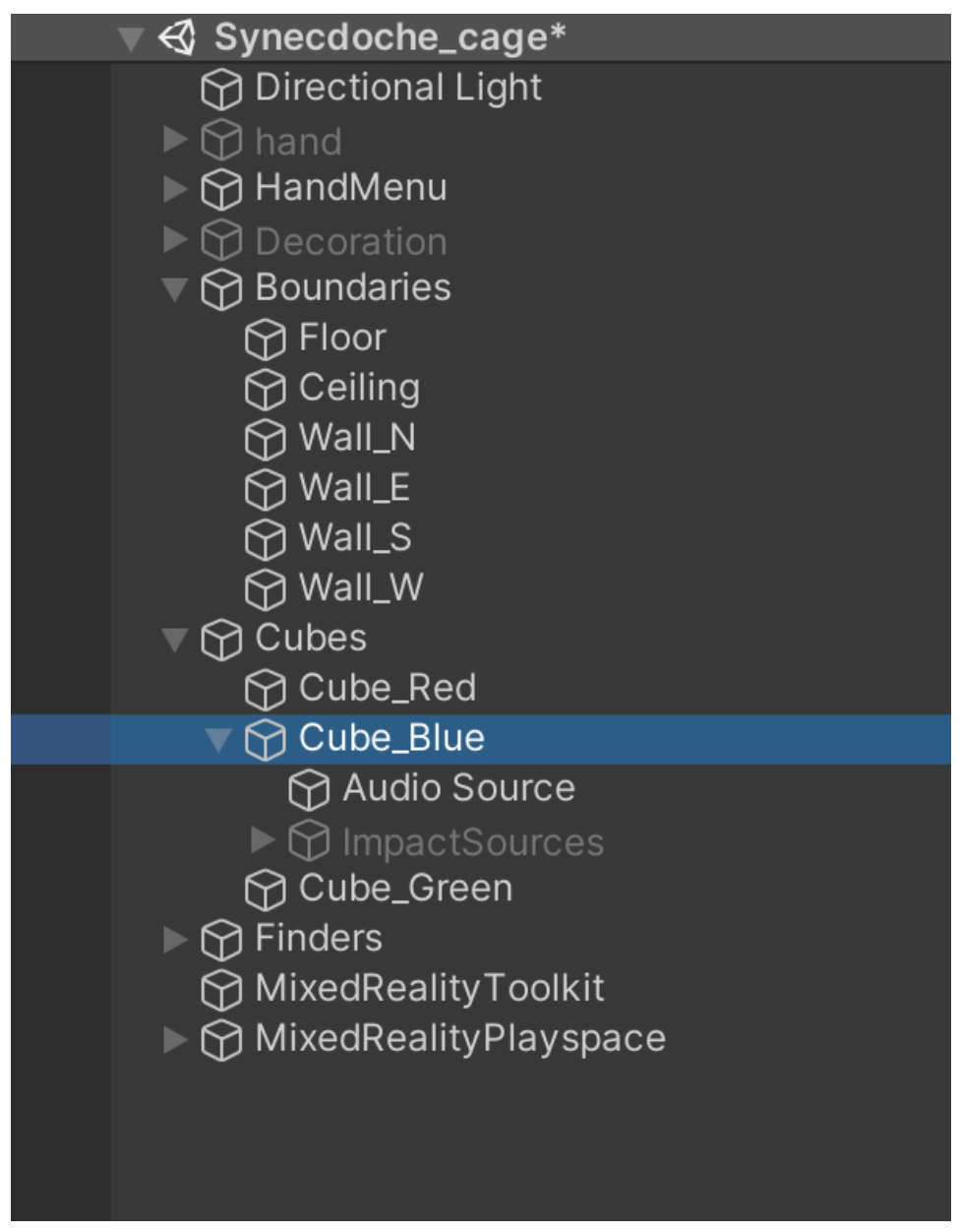

\section{SILENCE}

The Silence function is essentially the reverse of the Sing function, muting the various Audio Sources, and disabling ImpactTrigger.

Page | 141 


\section{FREEZE}

The Freeze function is essentially the reverse of the Release function, putting the Rigid Body components on the cubes to sleep and disabling the ObjectPuller, ObjectPusher, and gravity interactions.

\section{FIND}

The Find function generates a floating orb (that does not have a RigidBody collider) which will always point towards its appropriate cube, disappearing once the cube is in view.

\section{PING-PONG}

Finally, and this was more a happy accident than an intentional design, because of the way that I built the Hand Menus, they can be used like paddles to hit the cubes and send them floating off.

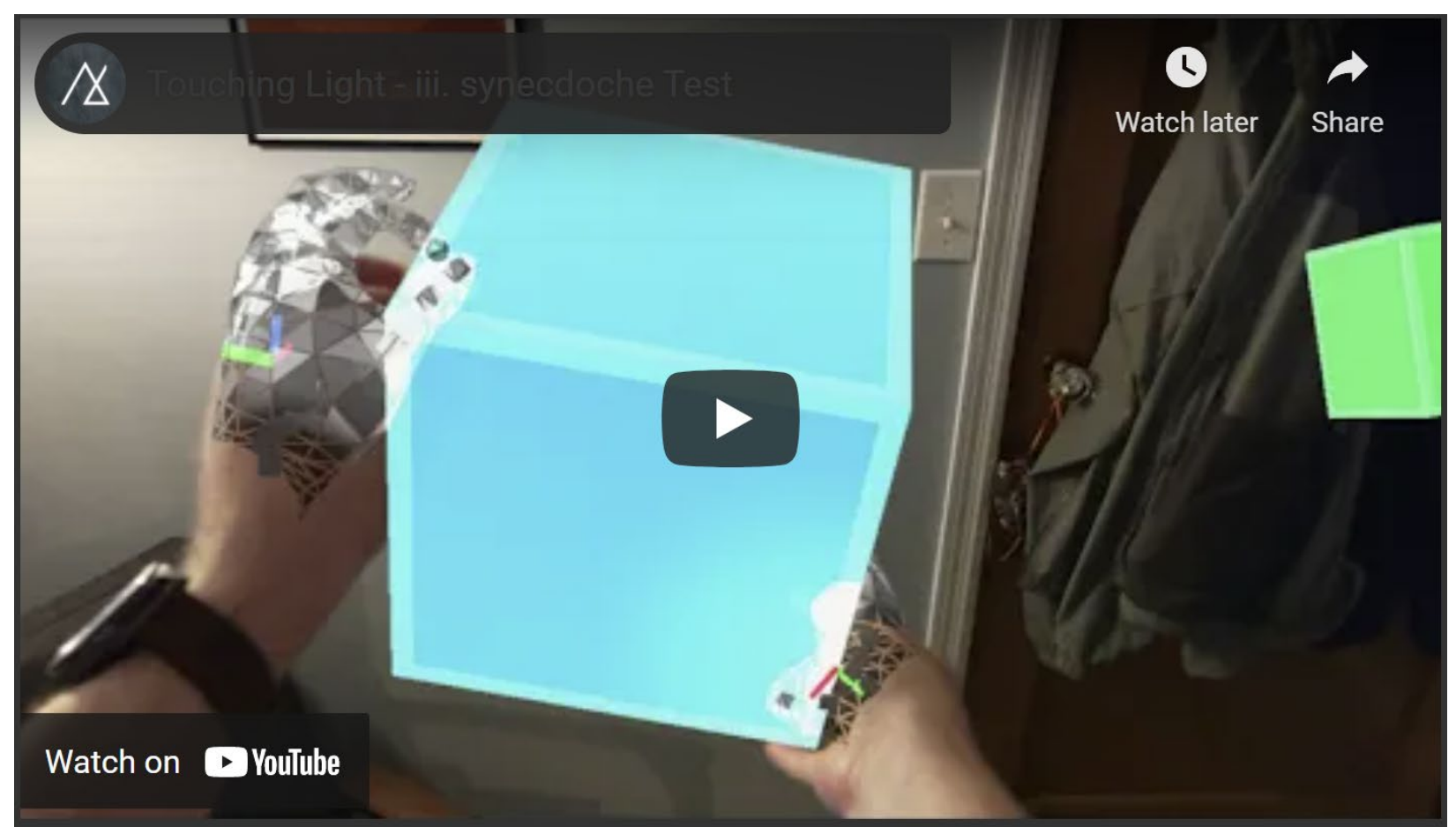

Overall, I pleased with the way that things are shaping up... recital is on May 1 at 10 AM EST! 


\section{gravity $(02 / 09 / 21)$}

Posted on February 9, 2021 (https://ianrileypercussion.com/2021/02/09/gravity/)

By ianthomasriley (https://ianrileypercussion.com/author/ianthomasriley/)

\section{In Uncategorized (https://ianrileypercussion.com/category/uncategorized/)}

With the spring semester at WVU in full force, finding the right balance between writing chapters for the dissertation, working on the Touching Light application, and teaching and coaching various lessons and ensembles has been tenuous, but so far, the project is still on track! With the first two 'movements' in a stable place and successfully ported to the HoloLens, the last piece of the puzzle is the development of the third and final movement of the application which involves both the most intractability as well as the most custom coding and design.

That said, the initial versions of the third movement have been picking up steam.

While the first movement aims to create a virtual representation of a physical object (a sound mixer), and the second movement creates an abstracted virtual representation of physical objects (the photosphere), the third movement explores interactions and functionality that is unique to the MR space by employing what I've referred to in my research as an 'esoteric liminality' (still not sold on this title, but it's what I'm working with at the moment). In English, this is simply a virtual environment that manifests and takes advantage of interactions that are unique to the MR platform, including interacting with abstract shapes (in our case, colored cubes), audio spatialization in an MR volume, and MR collisions of virtual and physical objects (this bit is proving to be a smidge tricky). 
Overall, the progress of development is on track, but as always, I'll need to remember that this is intended to be a proof of concept, not necessarily a polished, marketable experience (but it would be nice it if was, right?).

Details below about specific application features and how they've been achieved.

\section{The Barrier}

One of the biggest downsides to working in the Unity Editor (which would be the same for any development that is not done natively on the XR platform) is the inability for the IDE to map the physical environment in any meaningful way during development. To address this issue, I've simulated a physical room by using 3D places in a vaguely room-sized cube surrounding the initial instantiation of the MTRK camera, which allows any sort of collision interactions to be explored, even if it doesn't solve the issue of need to track collisions with the physical volume this will come soon. 


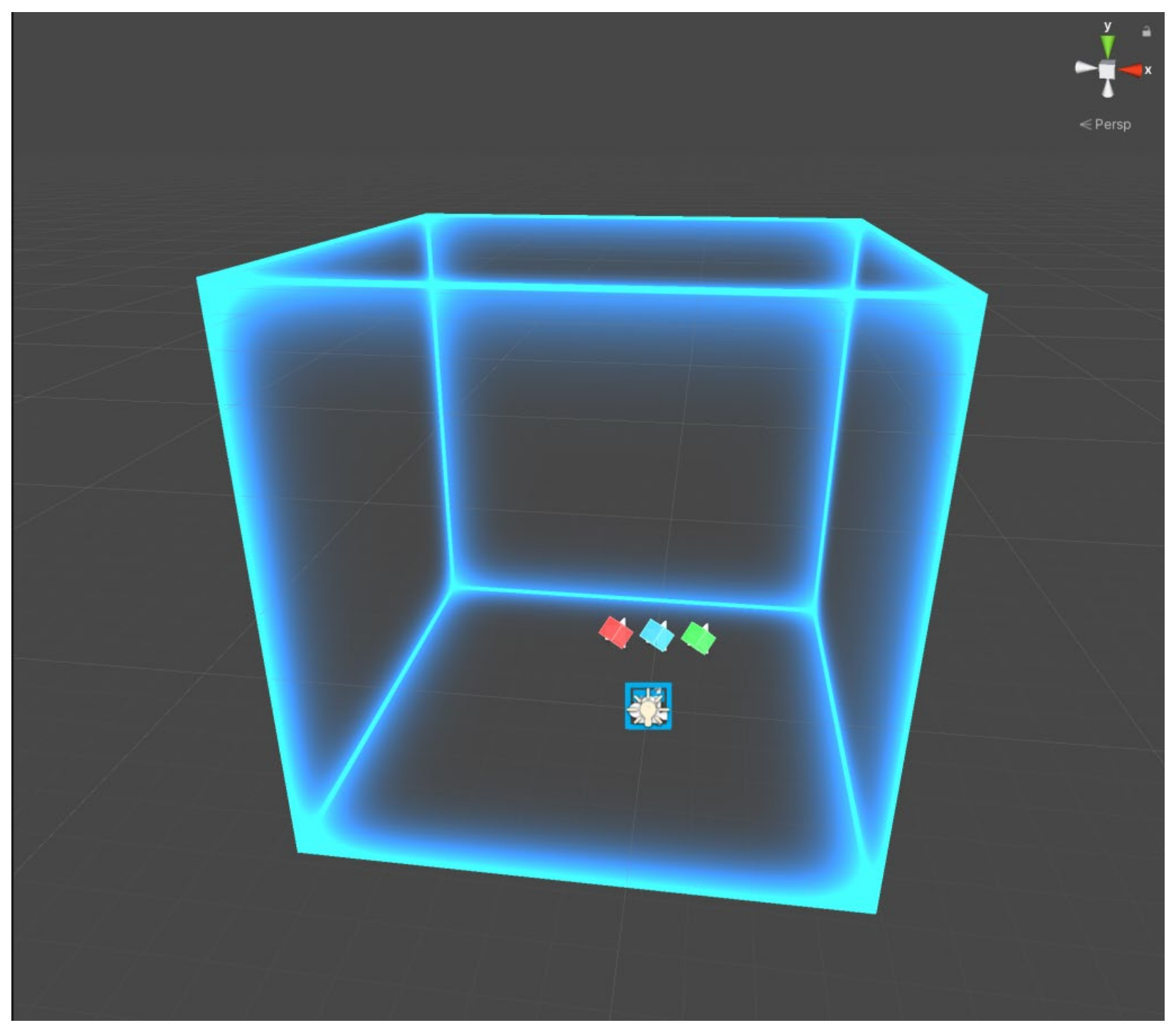

Figure 21: The Cüb

The cube represents the simulates physical volume, and acts as a barrier for virtual objects to collide with, like they would in a physical space with walls, floor, and ceiling. The coloration is simply for ease of identifying the edges of the barrier and will not be present in the final application. 


\section{A lot of Cubes}

With the barrier in place, I've devised three simply 'esoteric' objects to serve as prototypes for interactability which are represented by three smallish cubes, one red, one blue, and one green.

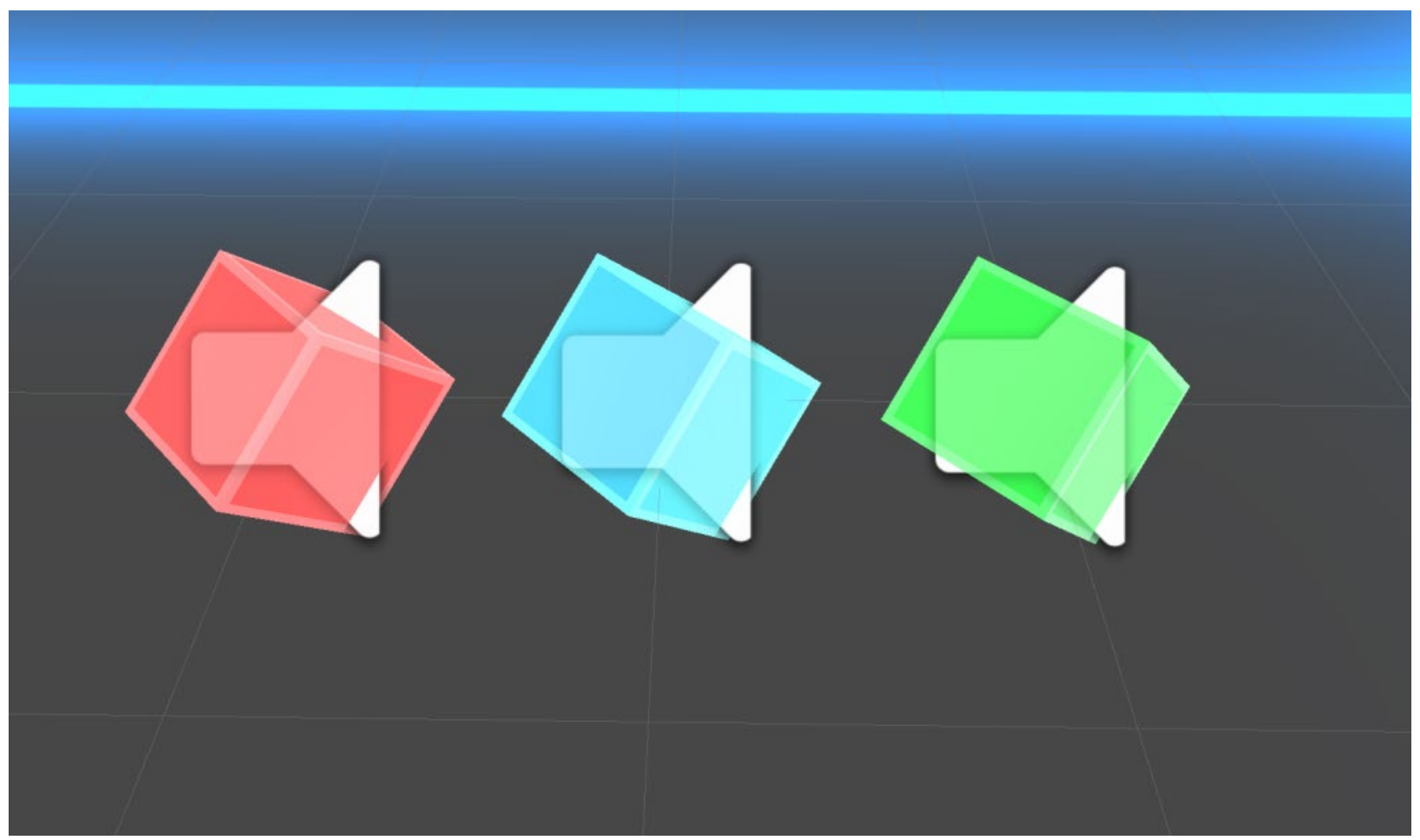

Figure 22: RGB cubes

At the moment, the cubes have three primary components: an spatialized audio source, interactability using hand rays and articulated hands, and new for this movement, rigidbody physics simulation. 


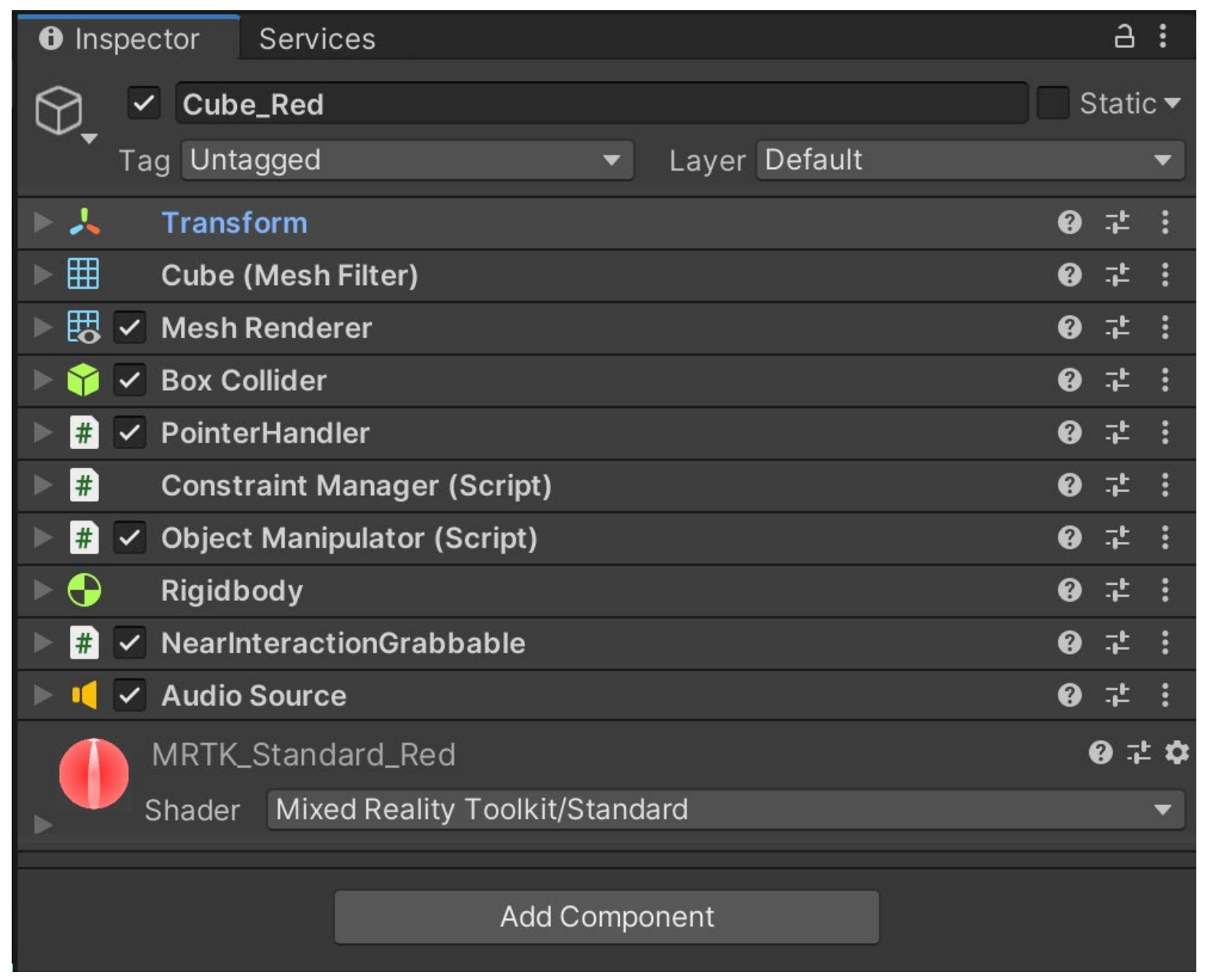

Figure 23: RedCube Inspector

\section{Grabbable}

Interactability was fairly straightforward and simply involved recreating the combination of components that I've used in the prior two movements; both the mixer and the photosphere have Object Manipulator and NearInteractionGrabbable scripts tied to them. 


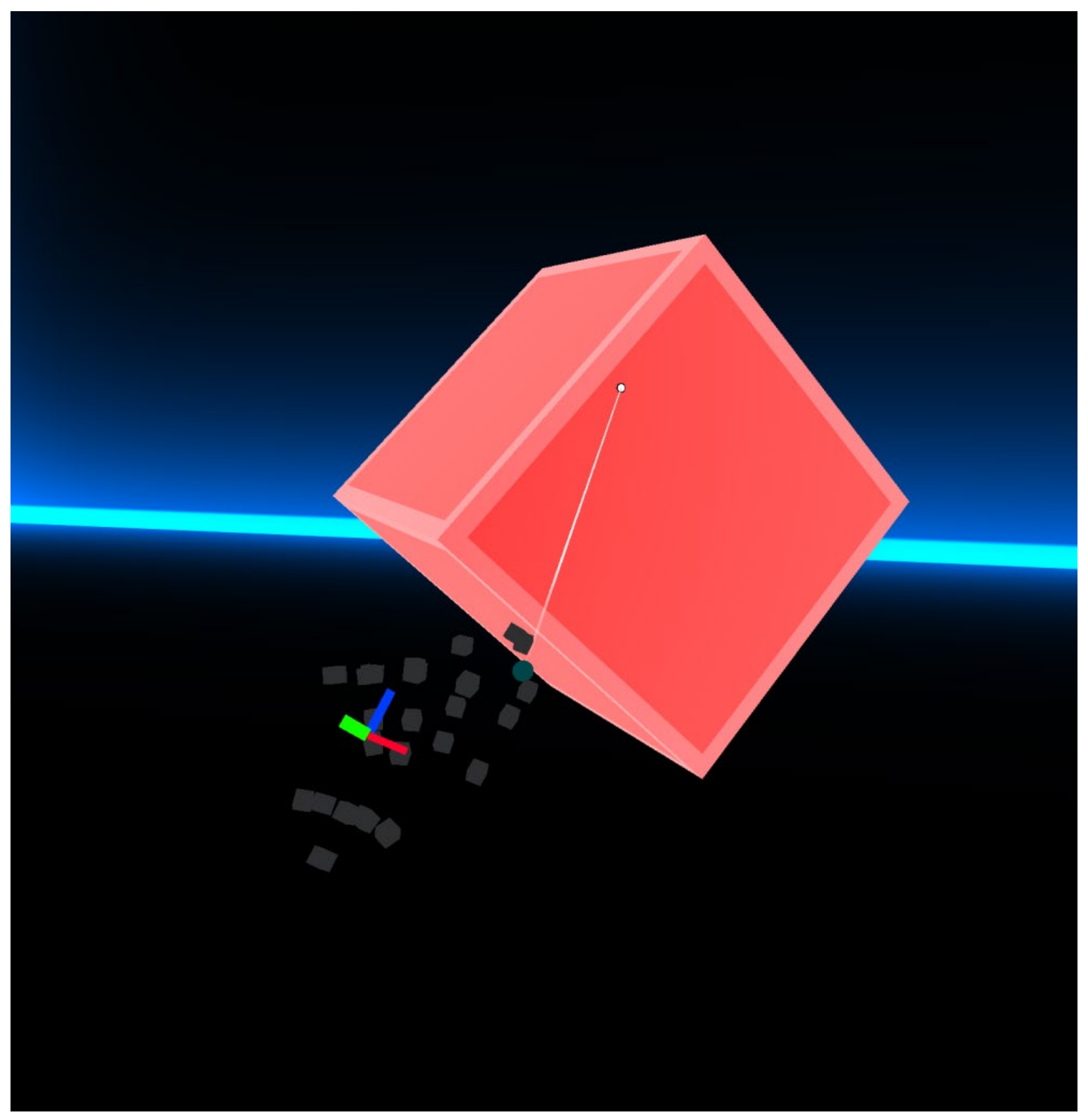

Figure 24: Using the simulated hand articulations

\section{Spatialized}

Secondarily, each cube serves as an audio source for one part of the three-part pre-recorded track (that I am still in the process of writing). For the moment, the bass, kick, and lead from movement one are assigned for testing purposes. Unique to these audio source components 
however is their spatialization. This means that the panning and volume of the individual tracks is determined by the user orientation and distance to the object; the closer the user is to the cube, the louder the volume of the track. Similarly, if the user places the cube to their right or left, the audio will be sent only to the appropriate side of the stereo mix. 


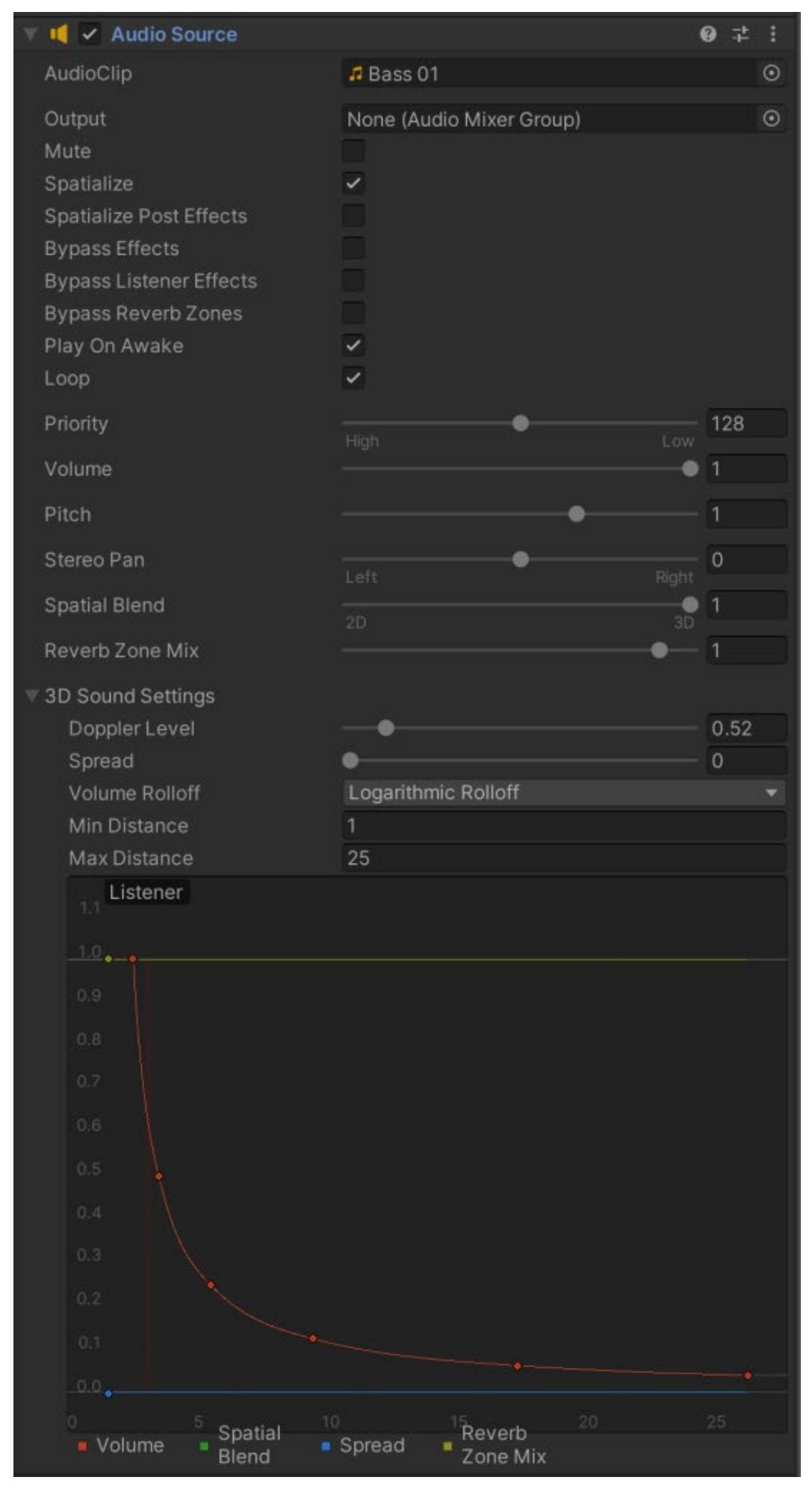

Figure 25: The audio spatializer

Page | 150 


\section{Weightless}

Spatialization by itself is a very interesting tool to play with, and I've had a lot of fun places the three cubes at different distances and orientations to create unique mixes, both in terms of volume and panning. In some ways, this concept is similar to the traditional style of mixer present in the first movement, but speaking from experience, the interactable experience is, for lack of a better word, much more esoteric in this model.

Compounding the creative interest of the audio spatialization is perhaps the most engaging element of the movement in its current iteration, namely the rigidbody physics simulation. By identifying the cubes as 'rigidbodies' (a term in visual effects and 3D modeling that means that the physics simulation treats the object as a contiguous, immutable whole), it is a fairly straightforward process to get Unity (and by extension the HoloLens) to apply a desired physics simulation to the objects.

In this case, I wanted the cubes to move through the virtual volume as if they were in outer space, without gravity and with near-perfect conservation of momentum. A few changes to the physics settings later, and we suddenly have space cubes! 


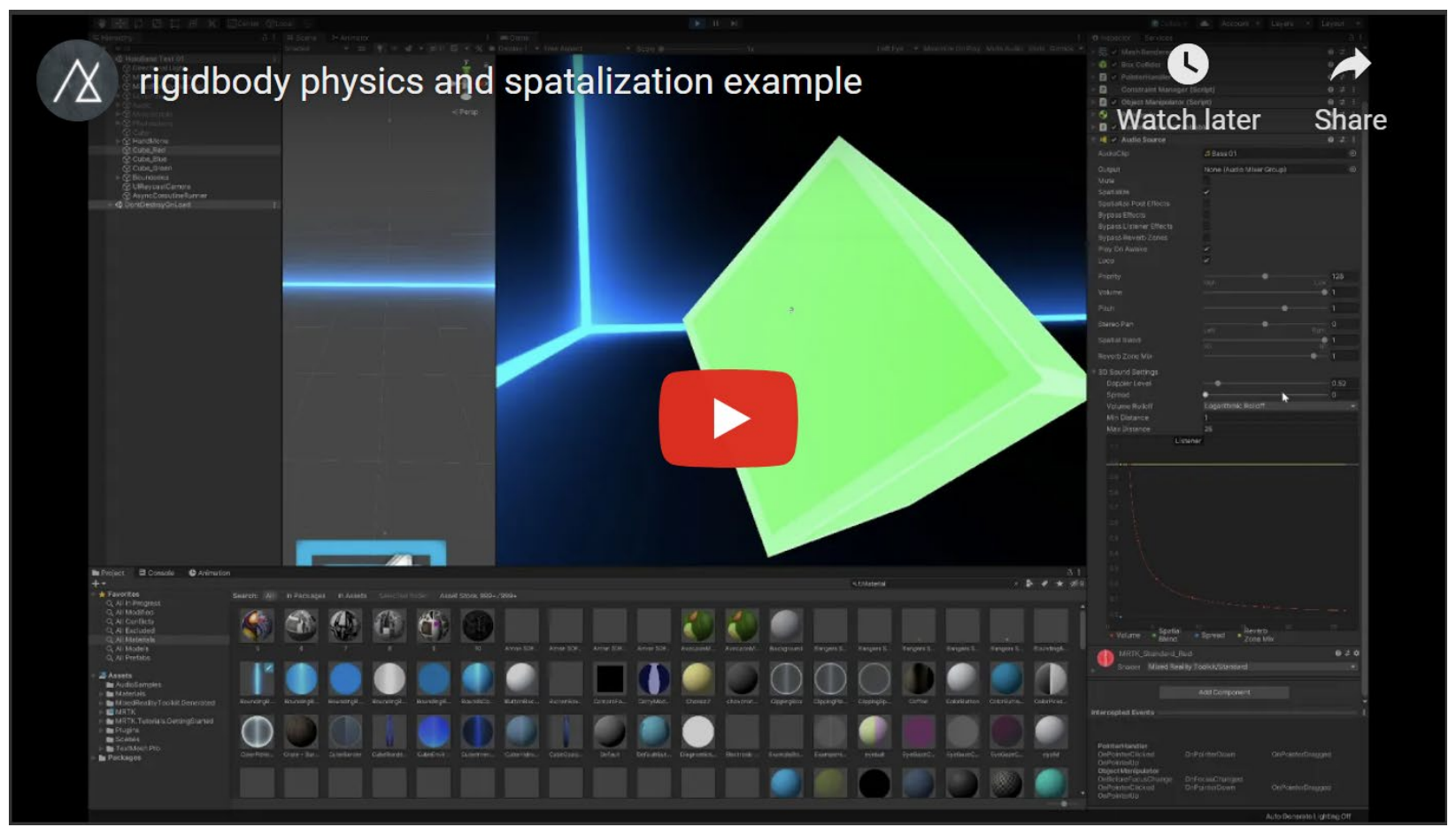

Figure 26: YouTube Video Preview - "rigidbody physics and spatialization example"

\section{(https://youtu.be/KhZrVhuCZ20)}

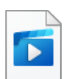

rigidbody physics and spatalization example.mp4

I'm not sure yet why the cubes seem to lose so much momentum when the strike a barrier; I've tried to play around with physic materials to add elasticity to the interactions, etc. but haven't had great luck so far. I'm sure there some relationship between the angular momentum and the static momentum that I'm missing, but even with that in mind there still seems to be an inordinate amount of velocity being shed after every collision. Ideally, the cubes will continue ricochet indefinitely, perpetually altering the spatialization of the track, but that's a story for another day.

\section{Using Hand Menus}


Apart from the volume and the cubes themselves, I've also implemented a contextual 'hand menu,' which is simply a menu whose appearance is determined by the gaze tracking on the HoloLens platform; if you are looking at the back of your right hand, the 'hand menu' should appear in the air toward the inside of the hand.

After 'losing' the cubes a few times, I thought it might be a good idea to have a menu that would be able to call simple commands like 'freeze,' 'mute,' 'reset,' etc. in the event that the cube(s) is for some reason unreachable. So far, the freeze and mute functions are working as their can be rigged to simple onClickEvent functions. The 'Reset All' command, as well as an as-yet uncreated 'Generate' command which will create cubes of certain colors will require some specific coding that I'm still getting a handle on (no pun intended). 


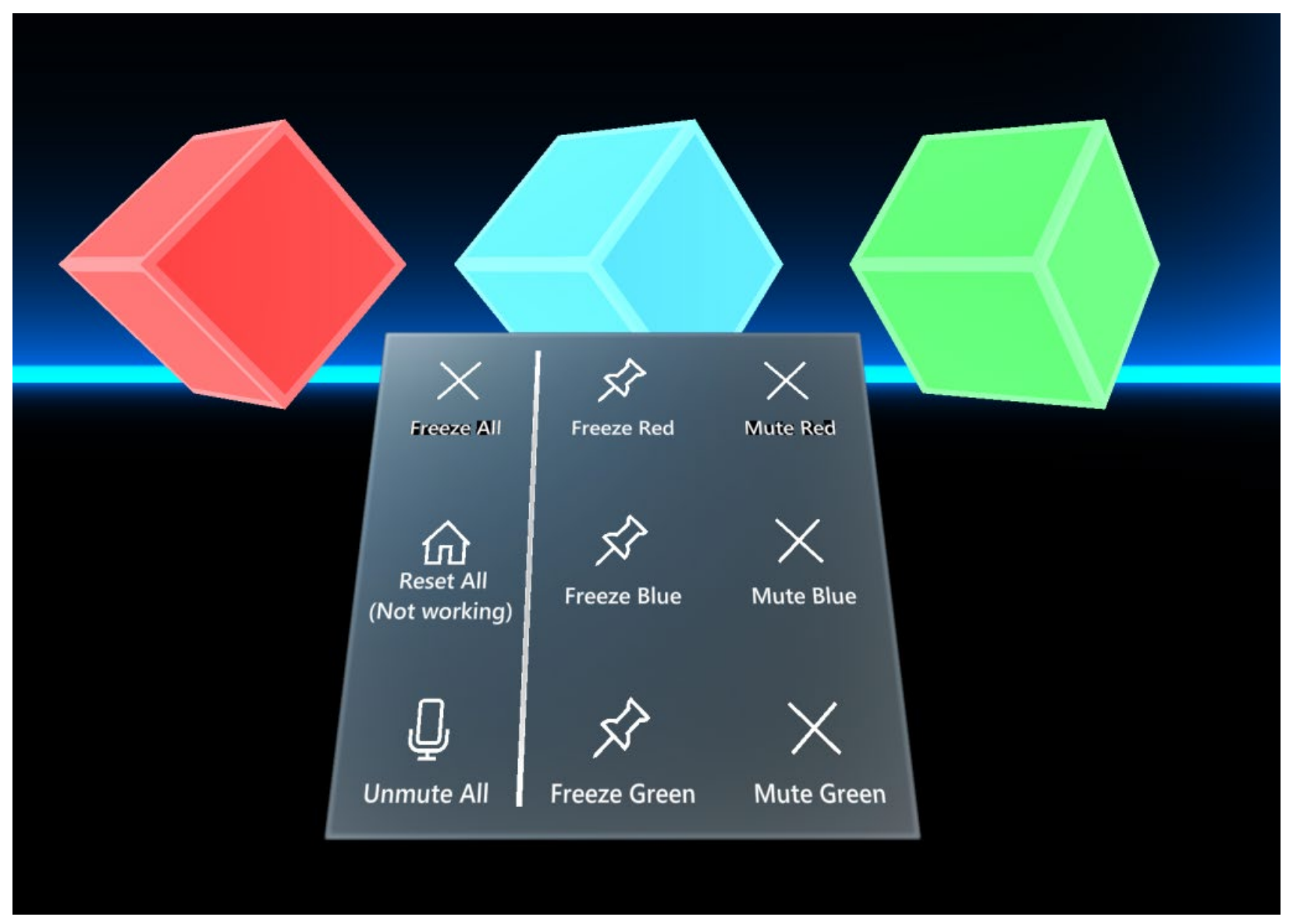

Figure 27: It's been difficult to get the 'hand menu' to show up without hands...

\section{Stretch Goals}

Depending on how long it takes to get the hand menu and the rigidbody interactions working the way that I'm intending, I also have some other ideas to map various other volumetric parameters to specific audio/mixer effects (e.g., size to effect pitch shifting, XYZ orientation to effect EQ, etc.), but that will take a back-seat to getting the initial interactions working smoothly. 


\section{radials $(01 / 26 / 21)$}

Posted on January 26, 2021 (https://ianrileypercussion.com/2021/01/26/radials/)

By ianthomasriley (https://ianrileypercussion.com/author/ianthomasriley/)

In touching-light (https://ianrileypercussion.com/tag/touching-light/)

Successfully deployed a stable build of Mixer Test 01 to the HoloLens 2 with good success; everything seemed to run smoothly, and the audio within sync with itself and adjustable using the programmed faders. The console itself is a bit awkward to move about as I did not include a way to view the bounding box, but with minimal trial and error I was able to place the mixer in a set location and successfully use the 'pin' function to lock it in place.

Continuing to stress test the app and clean up the code for the mixer app, but as it stands, with a successful deployment, that portion of the project is officially in alpha!

Chapters 1-3 of the dissertation have been submitted to my research advisor for a first pass at suggestions of editing and revisions and I have begun to reach out to some folks that I've had the opportunity to cross paths with to line up some interviews regarding their experience working with percussion and live technology, as well as to solicit their thoughts about specific elements of the project.

The second bit of programming will center around the development of a sort of adaptive photosphere which will rotate around the performer over a set duration to serve as a sort of inspirational score for improvisatory and/or pre-composed musical material.

A basic render of the current iteration in action below: 


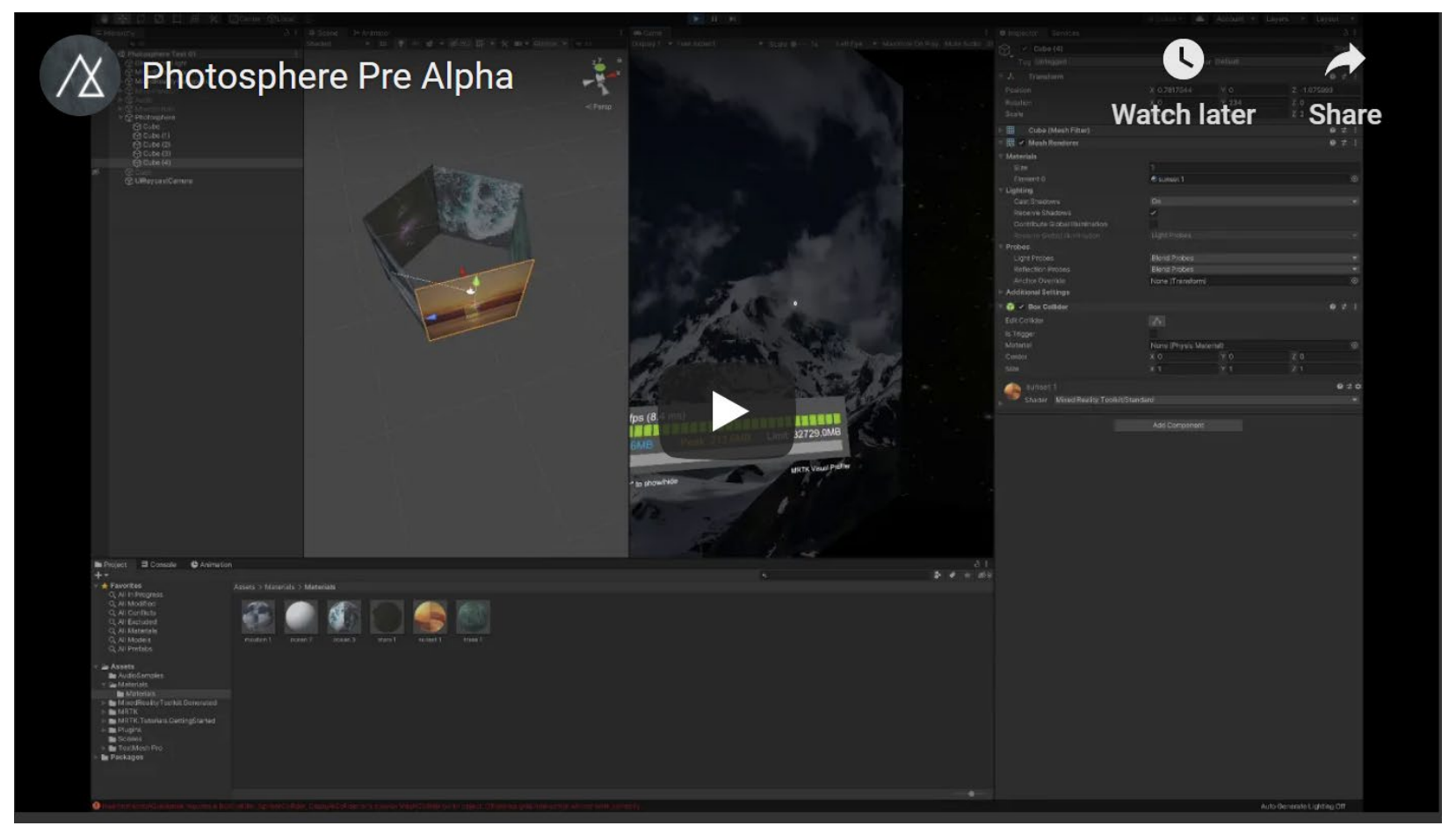

Figure 28: YouTube Video Preview - "Photosphere Pre Alpha"

\section{(https://youtu.be/O-HRHB7UJ98)}

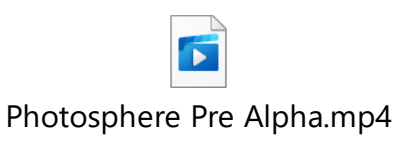

\section{Geometries}

At the moment, the images exist as grossly extrude 3D cubes, with little regard to the original dimensions of the images mapped onto them. This element will likely remain fairly similar (in terms of being size-agnostic) in the final build, but there will definitely be some tweaking regarding the overall size of the images, taking into account the fact that they would likely clip through the floor of the physical environment at their current scale, etc.

Ideally, I'll also get them to curve...! 


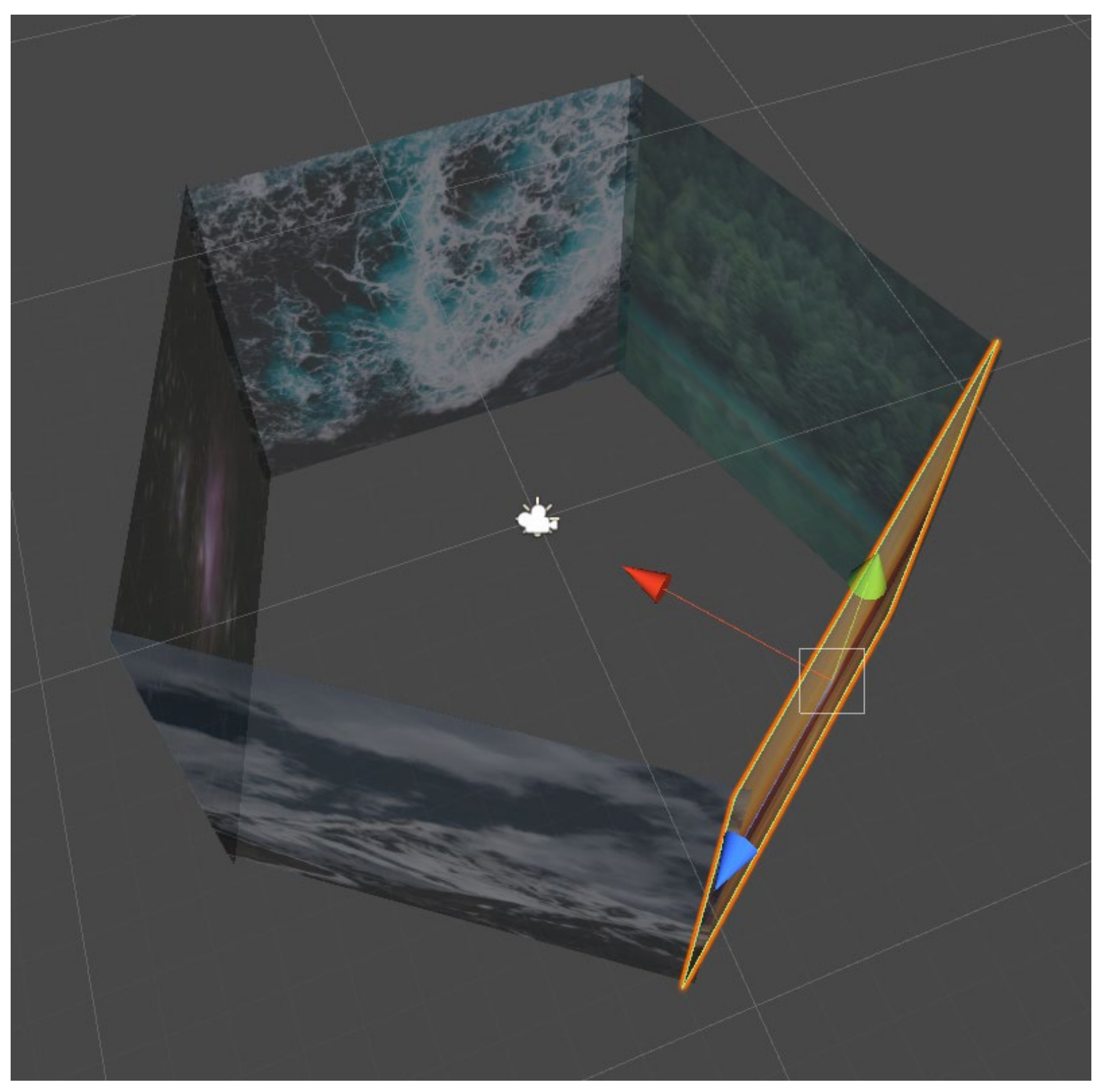

Figure 29: Photosphere 3D Model

\section{Textures}

Images are mapped directly as the albedo map for the 3D objects, bypassing the material creation structure native to the Unity editor; this might not be the right option, but for the moment, it lets things be visible when I need them to be. Currently the textures are approx. $50 \%$ translucent (using the RGBA alpha key mapped to the object opacity) to allow the performer to still be 
aware of the physical environment beyond the 'sphere' (e.g., the audience!), but the current clipping of the geometries combined with the texture wrapping create odd visuals like the double-sunset (which might be fixed by adjusting the Cull Mode to "Back" instead of "Off"). 


\section{Rendering Mode}

Transparent

Warning: Depth buffer sharing is enabled for this project, but this material does not write depth. Enabling depth will improve reprojection, but may cause rendering artifacts in translucent materials.

Fix Now

Cull Mode

Main Maps

- Albedo

Channel Map

\section{Albedo Alpha Mode}

Metallic

Smoothness

Normal Map

Emission

Triplanar Mapping

Tiling

Offset

Rendering Options

Directional Light

Specular Highlights

Spherical Harmonics

Reflections

Rim Light

Vertex Colors

Vertex Extrusion

Blended Clipping Width
Off

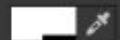

Transparency

Use the ClippingPrimitive component(s) to control other clipping properties.

Clipping Border

Near Fade

Fluent Options

Hover Light

Use the HoverLight component(s) to control Hover Light properties.

Override Color

Proximity Light

Border Light

Light Intensity

Round Comers

Inner Glow

Iridescence

Environment Coloring

Advanced Options

Render Queue Override

$\begin{array}{ll}X 1 & Y 1 \\ X 0 & Y 0\end{array}$

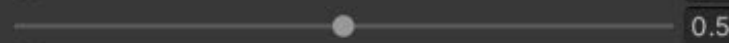

Render Queue

Enable GPU Instancing

Enable Stencil Testing

Figure 30: Photosphere Material Shader Example

Page | 159 


\section{Orientation}

Currently the panels are being drawn arbitrarily using a GridObjectCollection script, then tweaked individually to present the image-facing side toward the 'origin' (in this case, the center of the 'sphere.' 


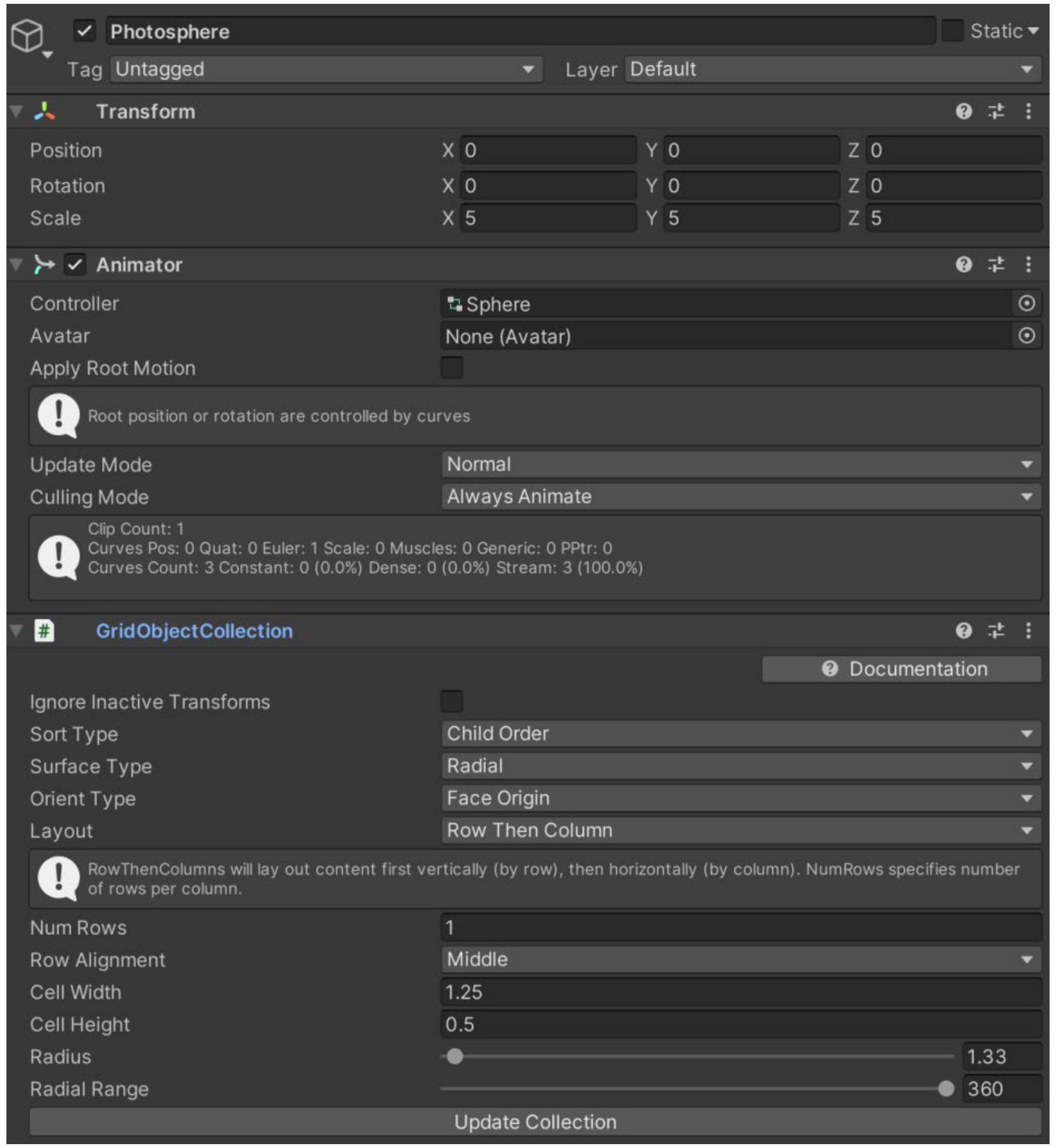

Figure 31: Photosphere Inspector

On execution, a HeadPositionOffset script also forces the 'sphere' to de-center from the perspective and facing of the user. 


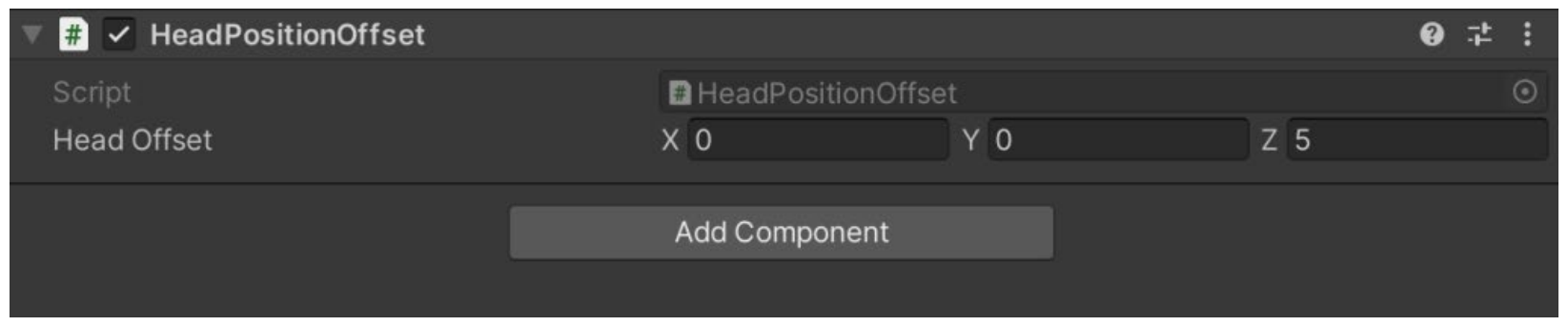

Figure 32: Photosphere HeadPositionOffset Component

Page | 162 


\section{sliders $(01 / 11 / 21)$}

Posted on January 11, 2021 (https://ianrileypercussion.com/2021/01/11/sliders/)

By ianthomasriley (https://ianrileypercussion.com/author/ianthomasriley/)

In Uncategorized (https://ianrileypercussion.com/category/uncategorized/)

I've spent the last two weeks banging my head against the proverbial wall, re-learning class and function indications in $\mathrm{CH}$, the programming language that underpins the Unity Engine (Unity), trying to get the Pinch Slider assets from the Microsoft's Mixed Reality Toolkit (MRTK) to talk to the AudioMixer class that's native to Unity.

With some help from a few professional programmers in my network, as well tutorials and manuals that have been developed by both Unity and Microsoft, as of this weekend, I have a functional skeleton for the first 'movement' of Touching Light, which involves independent audio faders that will adjust the volume of loop-based original music.

This will function as an augmentable backing track alongside which the performer can improvise freely, or engage with some pre-written melodic and harmonic material that will be presented with traditional staff-notation.

Final testing will occur in the next day or two to ensure that device deployment works as I am intending, after which I'll take a break from app development for the remainder of the week to shore up chapters 2 and 3 before sending it along to my research advisor for preliminary comments. 
Included below is a technical overview of the Unity assets and scripts involved at this juncture, which are subject to change as I optimize:

\section{In-Engine Render}

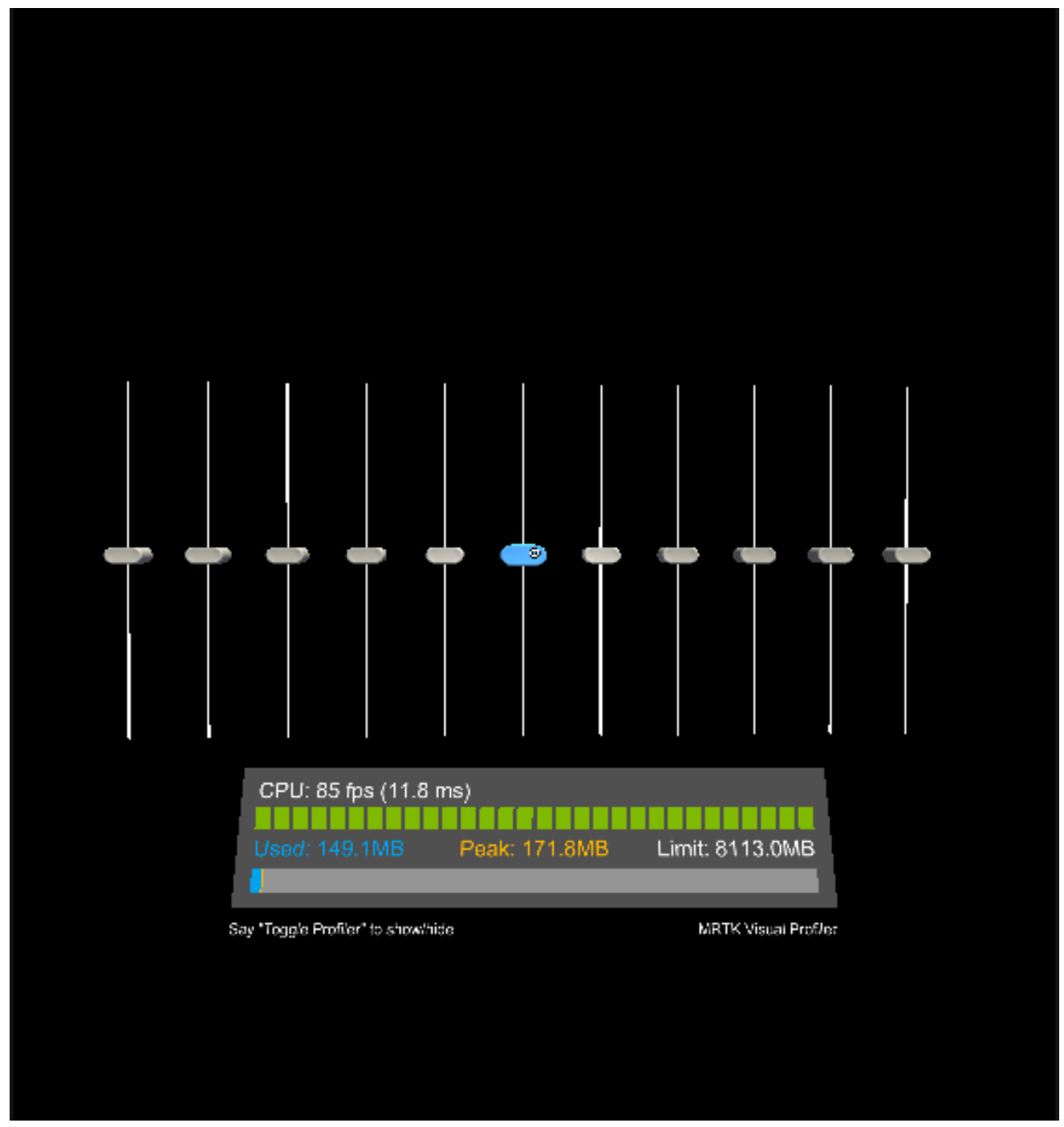

Figure 33: Mixer Version 1 Realtime Render

Here you're seeing a collection of 3D objects that have been adapted from the PinchSlider prefabricated (prefab) assets provided in the MRTK; each fader is interactable with the HoloLens 2 (HoloLens) “pinch” gesture (hence, "PinchSlider") and the 'thumb' (the knob that slides) will 
move vertically along that track, returning a value between 0 and 1, depending on where it is located along that track.

The context menu near the bottom is a profiler asset that allows me to track the CPU usage of different interactions in real-time, keeping an eye on whether or not things are in danger of causes lag, and freezing/crashing the program; so far, we're in the green.

PinchSlider Inspector 


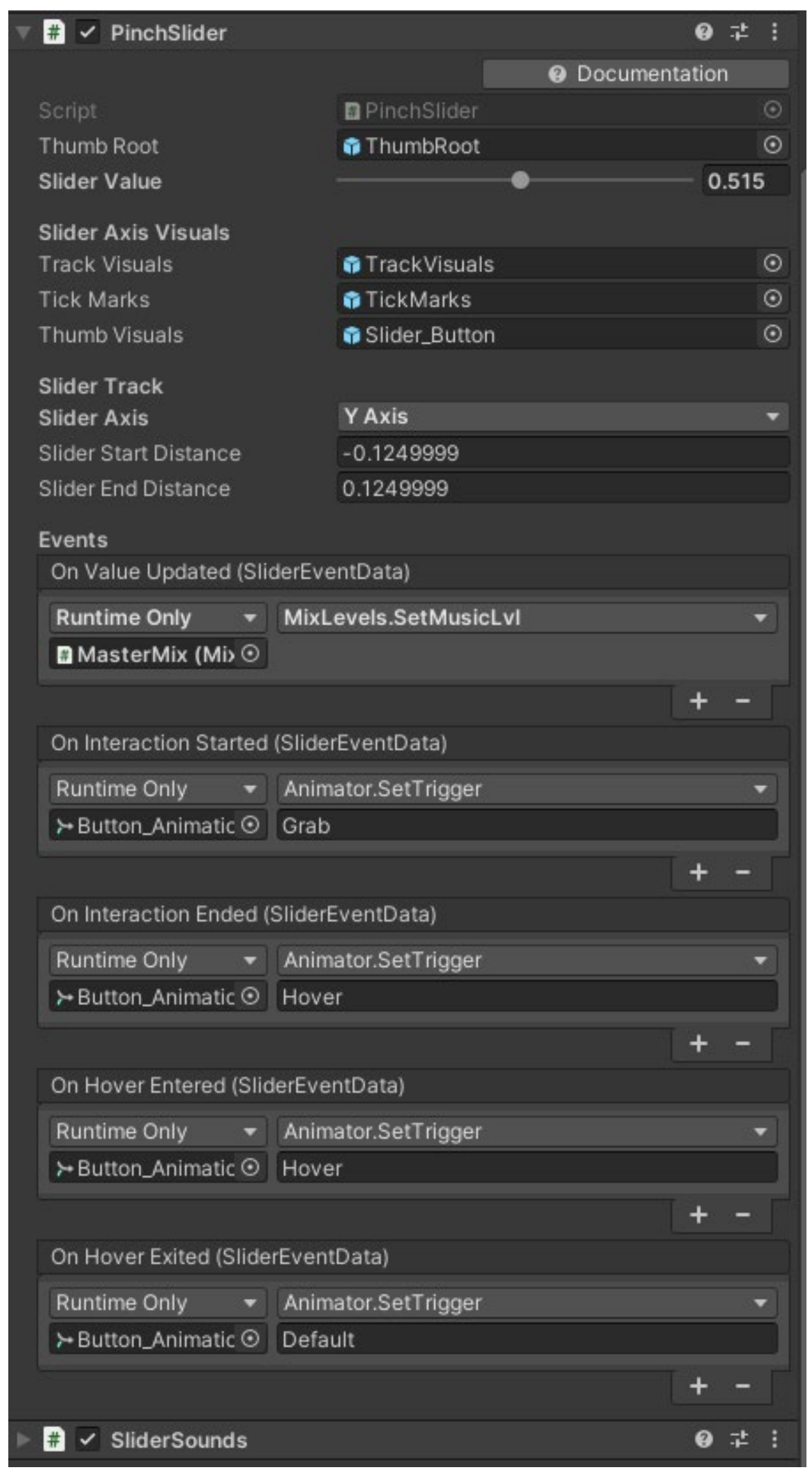

Figure 34: PinchSlider Inspector

Page | 166 
The connection between the PinchSlider asset exists within the 'Events' section: whenever the value of the slider (the number between 0 and 1 ) changes, the PinchSlider will return that value which can then be collected by other scripts (programs) and used to alter things like the volume of specific sounds, loops, etc.

I wrote the MixLevels.cs script (referenced in the On Value Updated event) to take that slider value and apply it to the volume for the appropriate track.

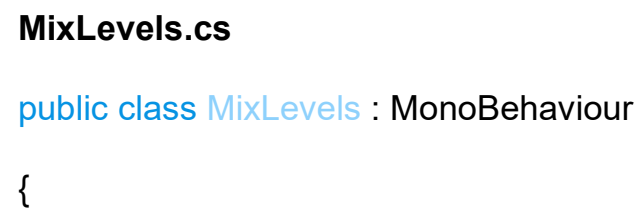




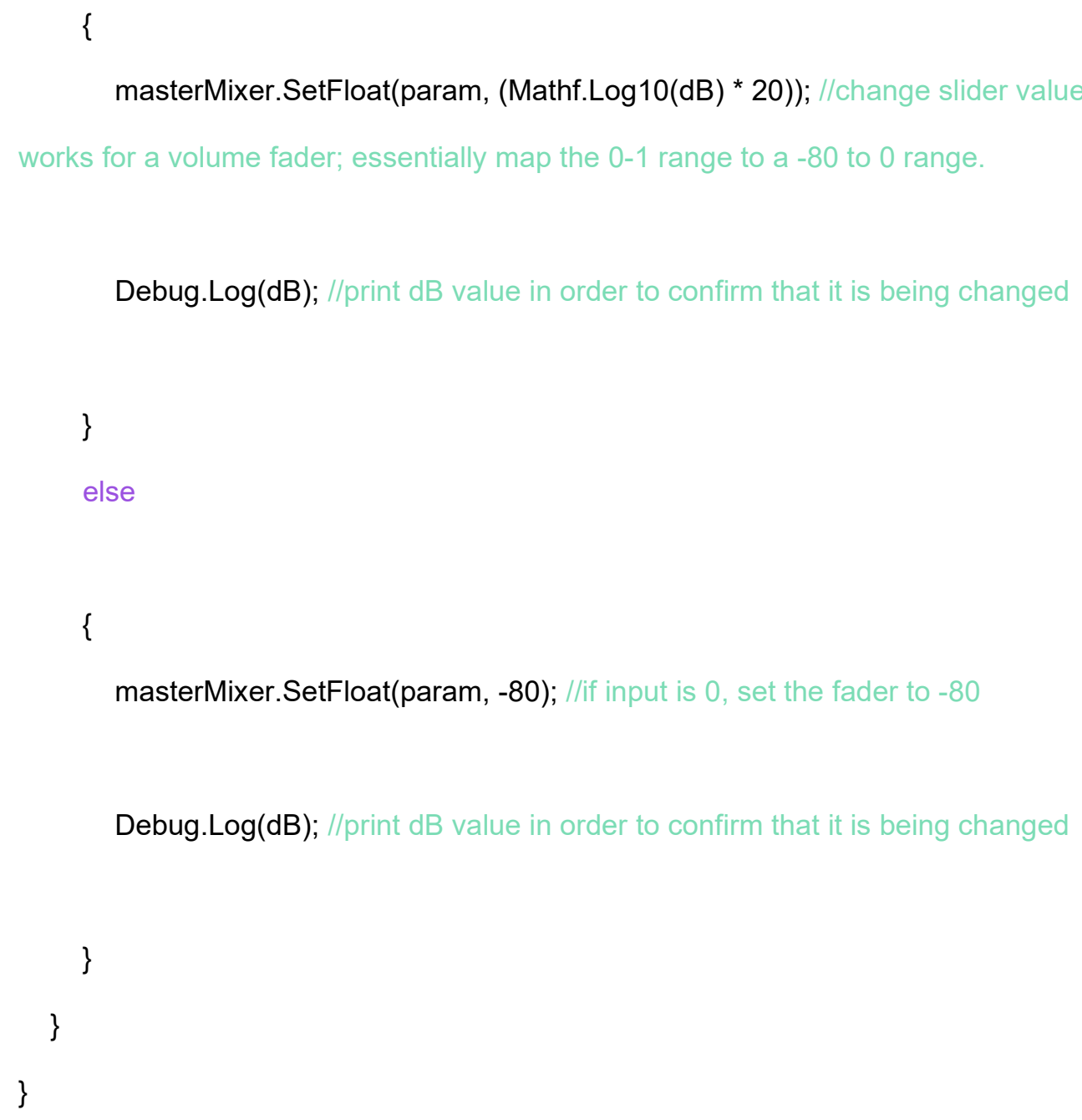

A fairly 'simple' script, as far as what is possible in the grand scale, this program allows for the user to input which fader's volume (an “exposed parameter," essentially meaning that it is visible and available for other programs to edit) this specific copy of the MixLevels.cs script should be attached to.

I've color-coded the code above; anything in sage green are 'comments;' they are notes within the program written in English for other programmers, that the computer ignores when it is running the program. Commenting is an important way to communicate with others who will look at your code in order to help them understand what your program is doing, and how. 
Master Fader

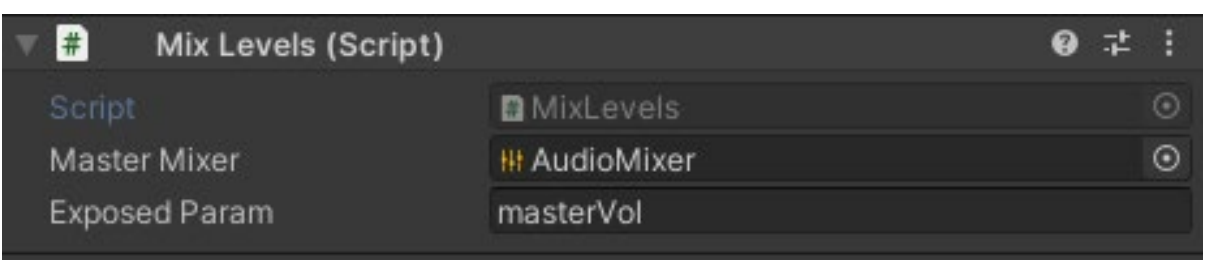

Figure 35: MixLevels.cs Inspector

While there is likely a more efficient and elegant way to create these connections, this is final piece of the puzzle: the place where the user can designate which audio track the slider should be in control of. You can see here that in the 'Exposed Param' field (which I've shown is a 'Serialized Field' in the script code above, thus prompting for an input) I've designated 'masterVol' which, as you might guess, is the reference to the Volume of the Master Fader (the one that controls the total overall volume of all of the tracks).

But multiplying this by five or ten times, I've then generated the necessary faders to control all 11 (10, plus the master fader) of the individual tracks that make up the complete original music. 


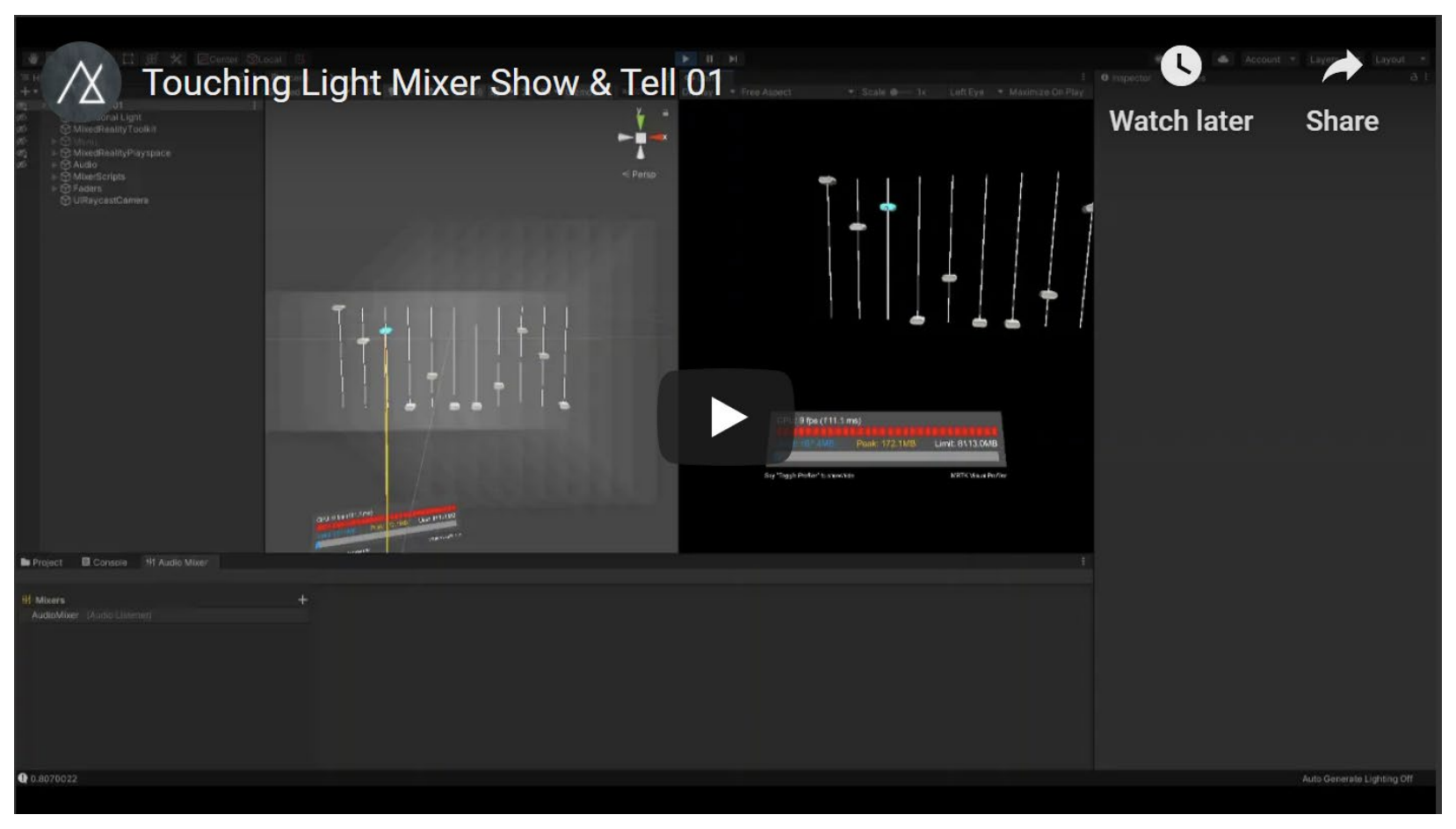

Figure 36: YouTube Video Preview - "Touching Light Mixer Show \& Tell 01 "

https://youtu.be/TkjQIsryXnU

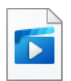

Touching Light Mixer Show \& Tell 01.mp4

Screen recording while rendering $3 \mathrm{D}$ and audio in real-time is a bit taxing (hence the red on the profiler) 
foundations $(12 / 14 / 20)$

Posted on December 14, 2020 (https://ianrileypercussion.com/2020/12/14/foundations/)

By ianthomasriley (https://ianrileypercussion.com/author/ianthomasriley/)

In touching-light (https://ianrileypercussion.com/tag/touching-light/)

Approval for the project prospectus (https://ianthomasriley.files.wordpress.com/2020/12/dma-

research-project-proposal-riley-ian.pdf) has come through from my DMA committee, and so it is time to properly begin this project. Today's task was to connect and deploy a Unity scene onto the Microsoft HoloLens 2 ("Lens") using the Microsoft Visual Studio ("VS").

It took some time and a bit of fiddling to get everything connected, but following some Microsoft ("MSFT") tutorials (https://docs.microsoft.com/en-us/windows/mixedreality/develop/platform-capabilities-and-apis/using-visual-studio) from their HoloLens documentation eventually proved successful.

The "foundations" scene that was successfully deployed is very basic: it is a gradient image that evokes a sense of a horizon line, rest approximately twelve inches away from the headset, and locked in orientation to always be directly in front of the Lens. The camera tracking allowed the image to maintain relative position (in relation to the Lens) while changing its absolute position (in relation to its coordinates in 3D space) and absolute orientation in response to the motion of the Lens, and by extension, my head.

The image was not interactable, and I eventually ended the deployment through VS on my laptop, as I had not given the Lens a way to quit out of the application once it had launched, though there may be something built into the MSFT Mixed Reality Toolkit ("MRTK") that I am not aware of. 
The next step will be to create a scene that is stationary that allows the camera to be track to the Lens, as well as determining how to run an application natively on the Lens without needing to launch and support it from VS.

\section{Page $\mid 172$}




\section{Glossary of Common Terms and Abbreviations}

Analog: In the context of this project, placed in opposition to 'digital;' used to describe objects, instruments, and practices that do not involve electronics/computers. A violin is an analog instrument.

Application: Refers to a discrete program that can be accessed through the user interface of a particular operating system to facilitate a particular set of user interactions.

Architecture: Refers broadly to the components that comprise applications and other computer programs, and specifically the way that they are created to work together.

Audio Interface: Refers to a piece of technology that allows an external microphone to be connected to a computer.

Augmented Reality (“AR"): Refers to technology that allows for interaction with virtual objects overlaid on a physical environment as rendered on a screen or other intermediary device (e.g., "Pokémon GO!”)

Computer-Generated Imagery ("CGI"): Refers to technology that allows artists and programmers to render images, including video, of virtual objects through the use of computer technology and specific software.

C-Sharp ("C\#"): Refers to a programming language; the syntax and vocabulary is specific to the language and serves as an intermediary between a user and a computer's machine code.

Digital: In the context of this project, used in opposition to 'analog;' used to describe objects, instruments, and practicing that involved and/or require the involvement of electronics/computers. A synthesizer is a digital instrument. 
Digital Audio Workstation ("DAW"): Refers to a piece of software that allows a user to interact with and edit live or pre-recorded audio signals, like the way that the same audio signals would be engaged with using an analog mixer.

Extended Reality (“XR"): Used to include all other "-R" (AR, MR, VR, etc.) terminology under a single unified umbrella.

Hardware: Refers to specific technology that forms the basis of a computer or other electronic device whose function is determined by the physical construction of the object. A computer is a piece of hardware.

Integrated Development Environment ("IDE"): Refers to the program(s) that serve as the primary interface within which another program is created; specifically, and IDE serves as a place to write, store, and compile computer code. Visual Studio or the Unity Editor are examples of an IDE.

Live Electronics: In the context of music-making, refers to specifical technologies that augment or alter some element of a live audio signal in real-time. A guitar delay pedal is an example of live electronics.

Microsoft HoloLens 2 ("HoloLens"): A standalone device that allows the user to engage with virtual objects in Mixed Reality, including hand-tracking and real-time environment mapping.

Mixed Reality ("MR"): Refers to technology that allows for interaction with virtual objects in physical 3-D space, wherein the virtual objects can interact and overlay physical objects, and the physical environment is not obscured (e.g., Microsoft HoloLens). 
Mixer: Refers to a piece of technology, analog or digital, that 'mixes' two or more audio signals, allowing a user to set specific volume levels for each individual signal, in addition to a number of other optional parameters. Both an analog mixing board and a DAW are examples of 'mixers.'

Microsoft Mixed Reality Toolkit (“MRTK"): Refers to a collection of assets and scripts developed by Microsoft to encourage best practices when developing applications for the HoloLens 2 specifically, and Microsoft Mixed Reality in general.

Operating System ("OS"): Refers to a piece or collection of software that serves as the framework upon which a computer system is designed. The OS serves as a sort of fundamental user interface for a computer or similar technology. Microsoft Windows and MacOS are both examples of operating systems.

Physical: In the context of this research, presented in opposition to 'virtual;' refers to real, tangible objects or environments that exist in the world around us. Both a desk chair and an office are 'physical.'

Real-time: Refers to software that updates in synchronous time with the interfacing user.

Software: Refers to specific technology that forms the basis of an operating system or other program whose function is determined by the formation of specific computer code. Microsoft Word is a piece of software.

Tethered/Untethered: Refers to the nature of a device and whether it requires a physical connection (a "tether," most commonly a cable connecting to a computer) to function. 
Unity Engine ('Unity'): Refers to a software development platform used in the creation of applications for Mixed Reality Environments.

Virtual: In the context of this research, presented in opposition to 'physical;' refers to imagined, or computer-rendered, intangible objects or environments that require some level of augmented, computer-assisted perception to interact with. A 360-degree picture and a video-game character are both 'virtual.'

Virtual Environment: Refers specifically to a volumetric space created through the conjunction of human perception and computer augmentation; has many of the same dimensions (length, height, depth, etc.) as a physical environment, but exists only as a construct within a computer or similar technology.

Virtual Reality ("VR"): Refers to technology that allows for interaction with virtual objects in a virtual 3-D space, wherein the virtual objects exist exclusively in a virtual environment and the physical environment is completely obscured (e.g., Facebook Oculus Quest).

Virtual Studio Technology ("VST"): Refers broadly to various synthesized instruments or audio editing techniques that are used in conjunction with DAWs to generate synthesized audio and/or music. 


\section{Bibliography}

“A History of Microsoft Windows | WIRED.” Accessed January 29, 2021. https://www.wired.com/2008/12/wiredphotos31/.

Abramo, Joseph Michael. "The Phantasmagoria of Competition in School Ensembles." Philosophy of Music Education Review 25, no. 2 (2017): 150-70. https://doi.org/10.2979/philmusieducrevi.25.2.04.

Adorno, Theodor W., and Susan Gillespie. "Music, Language, and Composition.” The Musical Quarterly 77, no. 3 (1993): 401-14.

“Adorno's Schreker: Charting the Self-Dissolution of the Distant Sound on JSTOR.” Accessed October 10, 2020 https://www.jstor.org/stable/4126440.

“Adorno's Wagner: History and the Potential of the Artwork on JSTOR.” Accessed October 10, 2020. https://www.jstor.org/stable/4489210.

Aggersberg, Brett. "A Critical Inquiry: Paintbrush to Pixels; Developing Paradigms in the Production and Consumption of New Media Art.” Ph.D., University of Wales Trinity Saint David (United Kingdom), 2017.

http://search.proquest.com/pqdtglobal/docview/1947628664/abstract/627B116B7F6A4F3 $\underline{8 \mathrm{PQ} / 2}$.

Baran, Michael, Nicole Lehrer, Margaret Duff, Vinay Venkataraman, Pavan Turaga, Todd Ingalls, W. Zev Rymer, Steven L. Wolf, and Thanassis Rikakis. “Interdisciplinary Concepts for Design and Implementation of Mixed Reality Interactive 
Neurorehabilitation Systems for Stroke.” Physical Therapy 95, no. 3 (March 2015): 44960. https://doi.org/10.2522/ptj.20130581.

Barone, Joshua. "Classical Music Meets Virtual Reality.” New York Times 168, no. 58380 (July 6, 2019): C6-C6.

Birhanu, Amare. "Interactive AR Experiences as Training Applications: Guidelines and Requirements for Piano Pedagogy in Mixed Reality.” M.S., Drexel University, 2017. http://search.proquest.com/pqdtglobal/docview/1946184261/abstract/8BE4B9A3B77140 $\underline{\mathrm{A} 8 \mathrm{PQ} / 2}$.

Blevins, Susanne Brenta. "From Corporeality to Virtual Reality: Theorizing Literacy, Bodies, and Technology in the Emerging Media of Virtual, Augmented, and Mixed Realities.” Ph.D., The University of North Carolina at Greensboro, 2017. http://search.proquest.com/pqdtglobal/docview/1927669599/abstract/8BE4B9A3B77140 $\underline{\mathrm{A} 8 \mathrm{PQ} / 5}$.

Bouënard, A., S. Gibet, and M. Wanderley. "Hybrid Inverse Motion Control for Virtual Characters Interacting with Sound Synthesis.” Visual Computer 28, no. 4 (April 2012): 357-70. https://doi.org/10.1007/s00371-011-0620-9.

Brandon Sanderson. Microsoft HoloLens: Welcome to the Team, 2016. https://www.youtube.com/watch?v=C98qaPAMVQU.

Çamc1, Anıl, and Rob Hamilton. "Audio-First VR: New Perspectives on Musical Experiences in Virtual Environments." Journal of New Music Research 49, no. 1 (January 2020): 1-7. https://doi.org/10.1080/09298215.2019.1707234. 
Chang, Wei-Chiao, Li-Wei Ko, Kuen-Han Yu, Yu-Chun Ho, Chia-Hsin Chen, Yuh-Jyh Jong, and Yi-Pai Huang. "EEG Analysis of Mixed-reality Music Rehabilitation System for Post-stroke Lower Limb Therapy.” Journal of the Society for Information Display 27, no. 6 (June 2019): 372-80. https://doi.org/10.1002/jsid.787.

Changwei Wang, Xuelei Zhang, and Lihua Liu. "The Framework of Simulation Teaching System for Sports Dance Based on Virtual Reality Technology.” Revista de La Facultad de Ingenieria 32, no. 15 (December 9, 2017): 530-36.

Channell, Timothy L., and Dennis M. Anderson. "Creating Virtual Internships in the Music Business." MEIEA Journal 10, no. 1 (January 2010): 173-83. https://doi.org/10.25101/10.9.

Chirico, Andrea, Patrizia Maiorano, Paola Indovina, Carla Milanese, Giovan Giacomo Giordano, Fabio Alivernini, Giovanni Iodice, et al. "Virtual Reality and Music Therapy as Distraction Interventions to Alleviate Anxiety and Improve Mood States in Breast Cancer Patients during Chemotherapy." Journal of Cellular Physiology 235, no. 6 (June 2020): 5353-62. https://doi.org/10.1002/jcp.29422.

Ciciliani, Marko. "Virtual 3D Environments as Composition and Performance Spaces.” Journal of New Music Research 49, no. 1 (January 2020): 104-13. https://doi.org/10.1080/09298215.2019.1703013.

“Comparison of Virtual Reality Headsets.” In Wikipedia, October 19, 2020. https://en.wikipedia.org/w/index.php?title=Comparison_of_virtual_reality_headsets\&oldi $\underline{\mathrm{d}=984283032 .}$. 
Cook, Gary D. Teaching Percussion, Enhanced, Spiral Bound Version. 003 Edition. Cengage Learning, 2018.

Csikszentmihalyi, Mihaly. Flow: The Psychology of Optimal Experience. 1st Edition. HarperCollins e-books, 2008.

"Daydream." Accessed January 29, 2021. https://arvr.google.com/daydream/.

Dingman, Hayden. “Rock Band VR: Rock Band's Roaring PC Debut Showcases Oculus Touch's Potential." PCWorld 35, no. 5 (May 2017): 125-31.

Du, Ruofei, and this link will open in a new window Link to external site. "Fusing Multimedia Data Into Dynamic Virtual Environments.” Ph.D., University of Maryland, College Park, 2018.

http://search.proquest.com/pqdtglobal/docview/2278078236/abstract/8BE4B9A3B77140 $\underline{\mathrm{A} 8 \mathrm{PQ} / 7}$.

Edison, Thomas. "Patent for Phonograph Recorder." Patent 42249 Summary. Industry Canada, June 15, 2015. https://www.ic.gc.ca/opic$\underline{\text { cipo/cpd/eng/patent/42249/summary.html?type=number_search\&tabs1Index=tabs1_1 }}$.

Elcott, Noam M. "The Phantasmagoric Dispositif:: An Assembly of Bodies and Images in Real Time and Space.” In Screen Space Reconfigured, edited by Susanne Ø. Sæther and Synne T. Bull, 283-316. Amsterdam University Press, 2020. https://doi.org/10.2307/j.ctv12pnt9c.15. 
“Even N00bs Can Rock Out on Magic Instruments’ New Guitar | WIRED.” Accessed January 11, 2021. https://www.wired.com/2016/04/even-n00bs-can-rock-magic-instruments-newguitar/.

"Flat Bayreuth: A Genealogy of Opera as Screened from Screen Genealogies: From Optical Device to Environmental Medium on JSTOR.” Accessed October 10, 2020. https://www.jstor.org/stable/j.ctvs32t6s.12.

Friedlander, Joshua P. “MID-YEAR 2019 RIAA MUSIC REVENUES REPORT,” n.d., 3.

Fusco, Katherine. "Voices from Beyond the Grave: Virtual Tupac's Live Performance at Coachella." Camera Obscura 30, no. 89 (May 2015): 28-53. https://doi.org/10.1215/02705346-3078314.

Gallet-Blanchard, Liliane. "VR "Montmartre in the Jazz Age"': The Problematics of Virtual Reality in Researching and Teaching Multicultural History.” Literary \& Linguistic Computing 20, no. 3 (September 2005): 313-25. https://doi.org/10.1093/1lc/fqi032.

Gallwey, W. Timothy. The Inner Game of Work: Focus, Learning, Pleasure, and Mobility in the Workplace. Reprint Edition. Random House Trade Paperbacks, 2001.

Gaugne, Ronan, Florian Nouviale, Octavia Rioual, Arnaud Chirat, Kevin Gohon, Vincent Goupil, Martin Toutirais, Bruno Bossis, and Valérie Gouranton. "EvoluSon: Walking through an Interactive History of Music.” PRESENCE: Teleoperators \& Virtual Environments 26, no. 3 (Summer 2017): 281-196. https://doi.org/10.1162/PRES_a_00298.

Gladwell, Malcolm. Outliers: The Story of Success, n.d. 
Glinsky, Albert. Theremin : Ether Music and Espionage. Urbana : University of Illinois Press, 2000. http://archive.org/details/thereminethermus00glin.

Gochfeld, David, Corinne Brenner, Kris Layng, Sebastian Herscher, Connor DeFanti, Marta Olko, David Shinn, Stephanie Riggs, Clara Fernández-Vara, and Ken Perlin. “Holojam in Wonderland: Immersive Mixed Reality Theater.” Leonardo 51, no. 4 (August 2018): 362-67. https://doi.org/10.1162/LEON a 01644.

Graham, Richard. “ENVIRONMENTAL HISTORIES AND PERSONAL MEMORY: Collaborative Works in Sonification and Virtual Reality.” Leonardo Music Journal 27, no. 1 (December 2017): 51-53. https://doi.org/10.1162/LMJ_a_01013.

Hamilton, Anita. "Be a Rock Legend in Your Living Room (No Guitar Required).” TIME Magazine 162, no. 13 (September 29, 2003): 78-78.

Harmon, Michael J. "Diffusion of Virtual Reality in Audiences Viewing Popular Music.” M.A., Sam Houston State University, 2018. https://search.proquest.com/pqdtglobal/docview/2061861273/abstract/720E359B1C284C $\underline{94 \mathrm{PQ} / 1}$.

Hass, Jeffrey. "Introduction to Computer Music.” Accessed September 16, 2020. https://cmtext.indiana.edu/index.php.

Heinrich Hertz, Heinrich Rudolph Hertz. Electric Waves: Being Researches on the Propagation of Electric Action With ... Macmillan, 1893. http://archive.org/details/electricwavesbe00jonegoog. 
Hertz, Heinrich, and William Thomson Baron Kelvin. Electric Waves: Being Researches on the Propagation of Electric Action with Finite Velocity Through Space. Macmillan, 1893.

Library of Congress, Washington, D.C. 20540 USA. "History of the Cylinder Phonograph | History of Edison Sound Recordings | Articles and Essays | Inventing Entertainment: The Early Motion Pictures and Sound Recordings of the Edison Companies | Digital Collections | Library of Congress.” Web page. Accessed January 5, 2021. https://www.loc.gov/collections/edison-company-motion-pictures-and-soundrecordings/articles-and-essays/history-of-edison-sound-recordings/history-of-thecylinder-phonograph/.

Holson, Laura M. “A C.G.I. James Dean? Some in Hollywood See 'an Awful Precedent' (Published 2019)." The New York Times, November 8, 2019, sec. Arts. https://www.nytimes.com/2019/11/07/arts/james-dean-cgi-movie.html.

Hornbostel, Erich M. von, and Curt Sachs. "Systematik Der Musikinstrumente. Ein Versuch." Zeitschrift Für Ethnologie 46, no. 4/5 (1914): 553-90.

Ideas. "How Design Is Helping to Democratize Music Making | Adobe XD Ideas." Accessed January 5, 2021. https://xd.adobe.com/ideas/perspectives/social-impact/how-designhelps-democratize-music-making/.

Howard-Hughes, Terri. "Coming Attractions: Immersive Digital Technologies in Cinema and Augmented, Mixed and Virtual Reality in Head Mounted Displays in the Twenty-First Century.” Ph.D., The University of Texas at Dallas, 2017. http://search.proquest.com/pqdtglobal/docview/1973160744/abstract/8BE4B9A3B77140 $\underline{\mathrm{A} 8 \mathrm{PQ} / 3}$. 
Huang, Andrew. This Was a Total Gamechanger for My Sound Design! YouTube Video, 2020. https://www.youtube.com/watch?v=GXQHiozYGTE.

Hugill, Andrew. "Internet Music: An Introduction.” Contemporary Music Review 24, no. 6 (December 2005): 429-37. https://doi.org/10.1080/07494460500296094.

Inc, Nielsen Business Media. "Tape/Audio/Video: High Technology Hits CES Audio Scene." Billboard, January 29, 1977.

Innocenti, Edoardo Degli, Michele Geronazzo, Diego Vescovi, Rolf Nordahl, Stefania Serafin, Luca Andrea Ludovico, and Federico Avanzini. "Mobile Virtual Reality for Musical Genre Learning in Primary Education.” Computers \& Education 139 (October 2019): 102-17. https://doi.org/10.1016/j.compedu.2019.04.010.

“It’s Better Virtual: AR/VR Takes the Stage - CES 2021.” Accessed January 29, 2021. https://www.ces.tech/Articles/2021/January/It\%E2\%80\%99s-Better-Virtual-AR-VRTakes-the-Stage.aspx.

Kedmey, Dan. "Virtually Real.” TIME Magazine 185, no. 4 (February 9, 2015): 12-12.

Kefauver, Alan P., and David Patschke. Fundamentals of Digital Audio, New Edition. A-R Editions, Inc., 2007.

Kenwright, Benjamin. “There's More to Sound Than Meets the Ear: Sound in Interactive Environments." IEEE Computer Graphics \& Applications 40, no. 4 (July 2020): 62-70. https://doi.org/10.1109/MCG.2020.2996371.

Kim-Boyle, David. “3D Notations and the Immersive Score.” Leonardo Music Journal 29 (December 2019): 39-41. https://doi.org/10.1162/lmj_a 01061. 
“LET'S DANCE: 'Grace’ Offers a Tantalizing Glimpse into Music Videos of the Future.” Computer Graphics World 40, no. 5 (October 9, 2017): 28-29.

Litterst, George F. "Random Recess: Music Teachers In The Twilight Zone.” American Music Teacher 67, no. 3 (January 12, 2017): 39-41.

Lombardo, Vincenzo, Andrea Valle, John Fitch, Kees Tazelaar, Stefan Weinzierl, and Wojciech Borczyk. “A Virtual-Reality Reconstruction of Poème Électronique Based on Philological Research.” Computer Music Journal 33, no. 2 (Summer 2009): 24-47. https://doi.org/10.1162/comj.2009.33.2.24.

Lucas, Jean-François, Tracy Cornish, and Todd Margolis. "To a Cultural Perspective of Mixed Reality Events: A Case Study of Event Overflow in Operas and Concerts in Mixed Reality." New Review of Hypermedia \& Multimedia 18, no. 4 (December 2012): 277-93. https://doi.org/10.1080/13614568.2012.746741.

“Magic Leap | Reality Is Just Beginning.” Accessed January 29, 2021. https://www.magicleap.com/.

Maxwell-Doherty, Kyle. "LINGUA FRANCA: THE USE OF LABANOTATION IN THE GESTURAL-BASED COMPOSITIONS OF WAYNE SIEGEL, MARK APPLEBAUM, AND CASEY CANGELOSI." University of Arizona, 2018.

“Maya Software | Computer Animation \& Modeling Software | Autodesk.” Accessed January 29, 2021. https://www.autodesk.com/products/maya/overview. 
McGrath, Sean, and Steve Love. "The User Experience of Mobile Music Making: An Ethnographic Exploration of Music Production and Performance in Practice." Computers in Human Behavior 72 (July 2017): 233-45. https://doi.org/10.1016/j.chb.2017.02.046.

“Microsoft HoloLens | Mixed Reality Technology for Business.” Accessed January 29, 2021. https://www.microsoft.com/en-us/hololens.

Twitter. "Microsoft HoloLens on Twitter." Accessed January 29, 2021. https://twitter.com/HoloLens/status/576531839856168960.

Miranda, Eduardo R. “Introduction: Leonardo Music Journal 29.” Leonardo Music Journal 29 (December 2019): 1-2. https://doi.org/10.1162/lmj_a_01053.

Morrow, Guy. "Conclusions: Virtual Reality, Augmented Reality and Mixed Reality.” Designing the Music Business, June 11, 2020, 177-201. https://doi.org/10.1007/978-3-030-48114$\underline{8} 8$.

Munoz, Isaac Garcia. “Space Time Exploration of Musical Instruments.” Ph.D., University of California, San Diego, 2020.

http://search.proquest.com/pqdtglobal/docview/2392577644/abstract/627B116B7F6A4F3 $\underline{8 \mathrm{PQ} / 1}$.

Nakagawa, Ryu, Ryo Komatsubara, Taku Ota, and Hidefumi Ohmura. Air Maestros: A MultiUser Audiovisual Experience Using MR. Sui'18: Proceedings of the 2018 Symposium on Spatial User Interaction, 2016. https://doi.org/10.1145/3267782.3274685.

"New Storytelling for Archaeological Museums Based on Augmented Reality Glasses from Communicating the Past in the Digital Age: Proceedings of the International Conference 
on Digital Methods in Teaching and Learning in Archaeology (12th-13th October 2018)

on JSTOR.” Accessed October 10, 2020. https://www.jstor.org/stable/j.ctv11cvx4t.12.

Oculus. Creating Spatialized Music for AR/VR, 2019.

https://www.youtube.com/watch?v=Owd0dbG76YM\&t=468s.

“Oculus Quest 2: Our Most Advanced All-in-One VR Headset | Oculus.” Accessed January 29, 2021. https://www.oculus.com/quest-2/.

Palumbo, Michael, Alexander Zonta, and Graham Wakefield. "Modular Reality: Analogues of Patching in Immersive Space.” Journal of New Music Research 49, no. 1 (January 2020): 8-23. https://doi.org/10.1080/09298215.2019.1706583.

Wave. "Past Waves." Accessed February 10, 2021. https://wavexr.com/past-waves/.

Pedersen, Karsten, Vedad Hulusic, Panos Amelidis, and Tim Slattery. "Spatialized Audio in a Custom-Built OpenGL-Based Ear Training Virtual Environment." IEEE Computer Graphics \& Applications 40, no. 5 (October 9, 2020): 67-81. https://doi.org/10.1109/MCG.2020.3013439.

Peire Serrate, Tomàs. "Volume I: Music in Virtual Reality: Musical Immersivity and Interactivity. Volume II: Meeting (1.0, 2.0 and 3.0) and Hillary (Concert and VR).” Ph.D., University of California, Los Angeles, 2019. https://search.proquest.com/pqdtglobal/docview/2331975391/abstract/AF1F442220B94F $\underline{8 \mathrm{APQ} / 1}$.

"Phantasmagoria and the Trump Opera from Critical Theory and Authoritarian Populism on JSTOR.” Accessed October 10, 2020. https://www.jstor.org/stable/j.ctv9hvtcf.14. 
Pinch, Trevor and Frank Trocco. Analog Days: The Invention and Impact of the Moog Synthesizer. Harvard University Press, 2002.

PlayStation. "PlayStation VR | Live the Game in Incredible Virtual Reality Worlds.” Accessed January 29, 2021. https://www.playstation.com/en-us/ps-vr/.

"Practice ... How?| Josh Gottry." Accessed October 25, 2020. https://gottrypercussion.com/practice-how/.

Pressing, Jeff. "Some Perspectives on Performed Sound and Music in Virtual Environments." PRESENCE: Teleoperators \& Virtual Environments 6, no. 4 (August 1997): 482. https://doi.org/10.1162/pres.1997.6.4.482.

Rabeler, Sylvia M. “A Spatial Color-Sound Model for Mixed Reality.” Kybernetes 40, no. 7/8 (August 9, 2011): 1119-28. https://doi.org/10.1108/03684921111160340.

Rasika Ranaweera, Michael Cohen, and Michael Frishkopf. "Narrowcasting and Multipresence for Music Auditioning and Conferencing in Social Cyberworlds.” PRESENCE: Teleoperators \& Virtual Environments 24, no. 3 (Summer 2015): 220-42. https://doi.org/10.1162/PRES_a 00232.

Rocchesso, Davide, Stefano Delle Monache, and Stephen Barrass. "Interaction by Ear." International Journal of Human-Computer Studies 131 (November 2019): 152-59. https://doi.org/10.1016/j.ijhcs.2019.05.012.

Rovelli, Carlo. The Order of Time. Riverhead Books, 2018.

Sachs, Curt. The History of Musical Instruments. Courier Corporation, 2006. 
Schulkin, Jay, and Greta B. Raglan. "The Evolution of Music and Human Social Capability." Frontiers in Neuroscience 8 (September 17, 2014). https://doi.org/10.3389/fnins.2014.00292.

Schutz, Michael, and Scott D Lipscomb. "INFLUENCE OF VISUAL INFORMATION ON AUDITORY PERCEPTION OF MARIMBA STROKE TYPES,” n.d., 5.

Serafin, Stefania, Federico Avanzini, Amalia De Goetzen, Cumhur Erkut, Michele Geronazzo, Francesco Grani, Niels Christian Nilsson, and Rolf Nordahl. "Reflections from Five Years of Sonic Interactions in Virtual Environments Workshops." Journal of New Music Research 49, no. 1 (January 2020): 24-34.

https://doi.org/10.1080/09298215.2019.1708413.

Serafin, Stefania, Cumhur Erkut, Juraj Kojs, Niels C. Nilsson, and Rolf Nordahl. "Virtual Reality Musical Instruments: State of the Art, Design Principles, and Future Directions." Computer Music Journal 40, no. 3 (Fall 2016): 22-40. https://doi.org/10.1162/COMJ a 00372.

"Sonic Dreamworlds: Benjamin, Adorno, and the Phantasmagoria of the Opera House from A Companion to the Works of Walter Benjamin on JSTOR.” Accessed October 10, 2020. https://www.jstor.org/stable/10.7722/j.ctt14brv7g.18.

Strickland, Tate. "Envisioning the Museum Voice: Gaze and Speech as Modes of Interacting with Art.” Master's, University of Washington, 2017. http://search.proquest.com/pqdtglobal/docview/1942901198/abstract/8BE4B9A3B77140 $\underline{\mathrm{A} 8 \mathrm{PQ} / 4}$. 
Stubley, Eleanor V. "The Performer, the Score, the Work: Musical Performance and Transactional Reading." Journal of Aesthetic Education 29, no. 3 (1995): 55-69. https://doi.org/10.2307/3333541.

Tatar, Kıvanç, Mirjana Prpa, and Philippe Pasquier. "Respire: Virtual Reality Art with Musical Agent Guided by Respiratory Interaction.” Leonardo Music Journal 29 (December 2019): 19-24. https://doi.org/10.1162/lmj a 01057.

“Technology, n.” In OED Online. Oxford University Press. Accessed January 5, 2021. http://www.oed.com/view/Entry/198469.

“The Computer Graphics Book Of Knowledge.” Accessed January 13, 2021. https://www.cs.cmu.edu/ ph/nyit/masson/history.htm.

“'The Music Is Not in the Notes, but in the Silence between.' - Google Search." Accessed February 11, 2021. https://www.google.com/search?q $=\% 22 \mathrm{The}+$ music + is + not + in + the + notes $\% 2 \mathrm{C}+$ but + in + th e+silence+between. $\% 22$.

Thomas, Kim. "Just Noticeable Difference and Tempo Change.” Psyencelab. Accessed October $26,2020$. http://psyencelab.com/uploads/5/4/6/5/54658091/just noticeable difference and tempo change.pdf.

Valve Corporation. “Upgrade Your Experience.” Accessed January 29, 2021. https://www.valvesoftware.com/en/index. 
Wang, Bin, Ruiqi Zhang, Chong Xi, Jing Sun, and Xiaochun Yang. "Virtual and Real-Time Synchronous Interaction for Playing Table Tennis with Holograms in Mixed Reality.” Sensors (14248220) 20, no. 17 (September 2020): 4857. https://doi.org/10.3390/s20174857.

Ward, Francis. "Technology and the Transmission of Tradition: An Exploration of the Virtual Pedagogies in the Online Academy of Irish Music.” Journal of Music, Technology \& Education 12, no. 1 (January 2019): 5-23. https://doi.org/10.1386/jmte.12.1.5_1.

Wave. "Wave | The Show Must Go Beyond." Accessed February 10, 2021. https://wavexr.com/.

"Wellingtons Sieg, Op.91 (Beethoven, Ludwig van) - IMSLP: Free Sheet Music PDF Download." Accessed February 3, 2021. https://imslp.org/wiki/Wellingtons_Sieg,_Op.91_(Beethoven,_Ludwig_van).

The National Endowment for the Humanities. "What Is Music?” Accessed February 11, 2021. https://www.neh.gov/humanities/2015/januaryfebruary/feature/what-music.

Williams, Alastair. "Technology of the Archaic: Wish Images and Phantasmagoria in Wagner on JSTOR.” Accessed October 10, 2020. https://www.jstor.org/stable/823710.

Wilson, Chris, and Michael Brown. "Sound, Space, Image and Music: Hybridity in Creative Process through Technology, Interactivity and Collaboration.” Journal of Music, Technology \& Education 5, no. 1 (May 2012): 89-107. https://doi.org/10.1386/jmte.5.1.89_1. 
Zakarin, Jordan. "It Took More CGI than You Think to Bring Carrie Fisher into The Rise of Skywalker.” SYFY WIRE, January 7, 2020. https://www.syfy.com/syfywire/it-tookmore-cgi-than-you-think-to-bring-carrie-fisher-into-the-rise-of-skywalker.

Zappi, Victor, Andrew Allen, and Sidney Fels. "Extended Playing Techniques on an Augmented Virtual Percussion Instrument.” Computer Music Journal 42, no. 2 (Summer 2018): 8 21. https://doi.org/10.1162/COMJ a 00457. 